\title{
Ruthenium-catalyzed hydrodefluorination with silane as the directing and reducing group
}

\author{
Huaquan Fang, ${ }^{\ddagger, a}$ Qiaoxing He, ${ }^{\ddagger, a, b}$ Guixia Liu, ${ }^{a, c}$ and Zheng Huang ${ }^{\star, a, b, d}$ \\ a State Key Laboratory of Organometallic Chemistry, Shanghai Institute of Organic \\ Chemistry, University of Chinese Academy of Science, Chinese Academy of Sciences, \\ 345 Lingling Road, Shanghai 200032, China \\ ${ }^{b}$ School of Physical Science and Technology, ShanghaiTech University, Shanghai \\ 201210, China \\ c Chang-Kung Chuang Institute, East China Normal University, Shanghai 200062 \\ dSchool of Chemistry and Material Sciences, Hangzhou Institute of Advanced Study, Uni- \\ versity of Chinese Academy of Sciences, 1 Sub-lane Xiangshan, Hangzhou 310024
}

\section{Supporting Information}

\section{Table of contents}

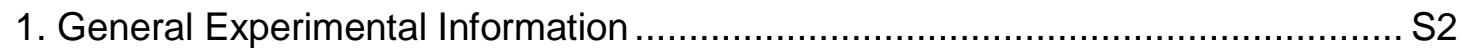

2. Synthesis of Polyfluorophenyl Hydrosilanes .................................................. S3

3. Ru(II)-Catalyzed ortho-Hydrodefluorination of Polyfluorophenyl Hydrosilanes ... S18

4. Elaboration of the HDF Products ............................................................ S41

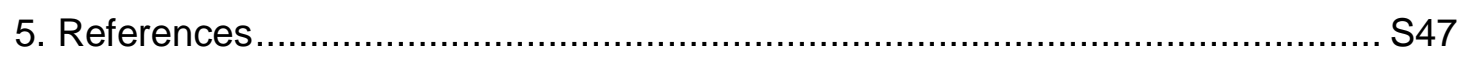

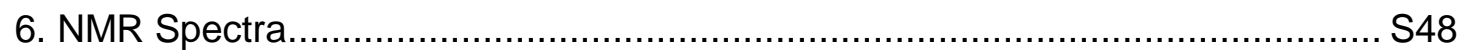




\section{General Experimental Information}

\section{a. Materials}

All manipulations were carried out in an argon-filled glovebox or under an atmosphere of dry argon using standard Schlenk techniques, unless otherwise stated. $n$ Hexane was purified by distillation over $\mathrm{CaH}_{2}$. Toluene, diethyl ether, and tetrahydrofuran (THF) were freshly distilled from sodium benzophenone ketyl immediately prior to use. The following chemicals were purchased and used as received: chlorodimethylsilane (Energy Chemical), dichloromethylsilane (Alfa Aesar), $\mathrm{Ru}_{3}(\mathrm{CO})_{12}(\mathrm{~J} \& \mathrm{~K})$, (cod)Ru(2-methylallyl) $)_{2}$ (J\&K), and $\mathrm{Ru}(\mathrm{acac})_{3}$ (TCl). (POCOP)RuH(NBD) (1a), (PCP)RuH(NBD) (1b), and (PSCOP)RuH(NBD) (1c) were prepared according to our previously reported procedures. ${ }^{1}\left({ }^{\mathrm{B} u P C P}\right) \mathrm{IrH}(\mathrm{Cl})(\mathbf{1} \mathbf{d})^{2}$ and $\left({ }^{(\mathrm{Pr} P C P}\right) \operatorname{lrH}(\mathrm{Cl})(\mathbf{1 e})^{3}$ were prepared according to published procedures. All other reagents were purchased from commercial sources and used as received, unless specified otherwise. Oil bath was used for all reactions that require heating.

\section{b. Methods}

NMR spectra were recorded on Agilent $400 \mathrm{MHz}$, Varian Mercury $400 \mathrm{MHz}$ spectrometer, and Bruker $400 \mathrm{MHz}$ spectrometer. ${ }^{1} \mathrm{H}$ NMR spectra were referenced to residual proton solvent peaks or tetramethylsilane signal $(0 \mathrm{ppm})$, and ${ }^{13} \mathrm{C}$ NMR spectra were referenced to the solvent resonance. Data are presented as follows: chemical shift, multiplicity $(\mathrm{s}=$ singlet, $\mathrm{d}=$ doublet, $\mathrm{t}=$ triplet, $\mathrm{q}=$ quartet, $\mathrm{m}=$ multiplet and/or multiple resonances), integration, coupling constant in hertz $(\mathrm{Hz})$. High resolution mass spectrometer (HRMS) was performed on the Analytical Laboratory of Shanghai Institute of Organic Chemistry (CAS).

\section{c. Attention}

All substrates were synthesized from bromopolyfluorobenzenes in a Grignard reaction, which is an exothermic process and must be treated with caution to prevent the potential violent heat release especially in large scale synthesis. 


\section{Synthesis of Polyfluorophenyl Hydrosilanes}

\section{Method A:}

$$
\begin{aligned}
& \text { i) } \mathrm{Mg} \text { (2.2 equiv), } \mathrm{I}_{2} \\
& \mathrm{HCl}_{2} \mathrm{SiMe} \text { (1.1 equiv) }
\end{aligned}
$$

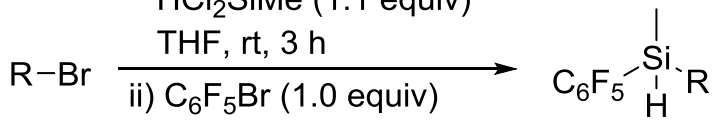

$$
\begin{aligned}
& \text { rt, overnight } \quad \mathrm{R}=\text { aryl or alkyl }
\end{aligned}
$$

To a mixture of magnesium turnings (2.2 equiv), a crystal of iodine, and dichloromethylsilane (1.1 equiv) in dry THF (30 mL), aryl bromide or alkyl bromide (1.0 equiv) was added dropwise at room temperature under Ar atmosphere. After stirring at room temperature for $3 \mathrm{~h}$, bromopentafluorobenzene (1.0 equiv) was added dropwise and the stirring was continued overnight. Afterwards, the reaction was quenched with saturated aqueous $\mathrm{NH}_{4} \mathrm{Cl}$ solution $(20 \mathrm{~mL})$ and extracted with $\mathrm{Et}_{2} \mathrm{O}$ three times $(20 \mathrm{~mL} \times 3)$. The combined organic layer was dried over $\mathrm{Na}_{2} \mathrm{SO}_{4}$, filtered, and concentrated under a reduced pressure. The residue was purified by flash column chromatography on silica gel with petroleum ether as the eluent to give the corresponding hydrosilanes.

\section{Method B:}

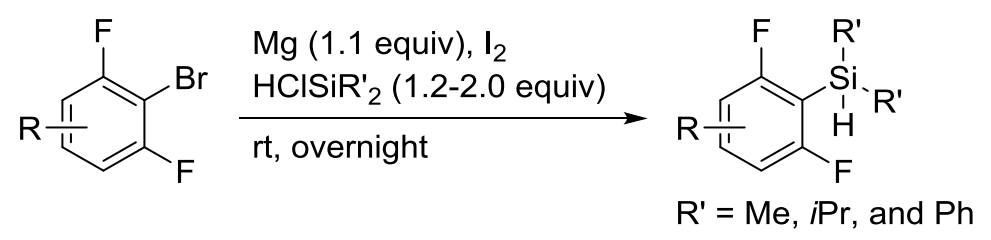

To a mixture of magnesium turnings (1.1 equiv), a crystal of iodine, and chlorodimethylsilane (2.0 equiv), chlorodiisopropylsilane (1.2 equiv), or chlorodiphenylsilane (1.2 equiv) in dry THF (30 mL), arylbromide (1.0 equiv) was added dropwise at room temperature under Ar atmosphere. After stirring at room temperature overnight, the reaction was quenched with saturated $\mathrm{NH}_{4} \mathrm{Cl}$ solution $(20 \mathrm{~mL})$ and extracted with $\mathrm{Et}_{2} \mathrm{O}$ three times (20 $\mathrm{mL} \times 3$ ). The combined organic layer was dried over $\mathrm{Na}_{2} \mathrm{SO}_{4}$, filtered, and concentrated under a reduced pressure. The residue was purified by flash column chromatography on silica gel with petroleum ether as the eluent to give the corresponding hydrosilanes. 


\section{Spectral data for the hydrosilanes}<smiles>C[Si](c1ccccc1)(c1ccccc1)c1c(F)c(F)c(F)c(F)c1F</smiles>

Methyl(perfluorophenyl)(phenyl)silane (2a). Bromobenzene $(2.1 \mathrm{~mL}, 20 \mathrm{mmol}$ ) and bromopentafluorobenzene $(2.5 \mathrm{~mL}, 20 \mathrm{mmol})$ was subjected to method A to give the corresponding hydrosilane as a colorless liquid $(0.77 \mathrm{~g}, 26 \%) . \mathrm{R}_{f}=0.9$ (petroleum ether). ${ }^{1} \mathrm{H}$ NMR $\left(400 \mathrm{MHz}, \mathrm{CDCl}_{3}\right): \delta 7.64(\mathrm{~d}, J=8.0 \mathrm{~Hz}, 2 \mathrm{H}), 7.48-7.40(\mathrm{~m}, 3 \mathrm{H}), 5.20-5.15(\mathrm{~m}$, $1 \mathrm{H}), 0.81-0.79(\mathrm{~m}, 3 \mathrm{H}) .{ }^{19} \mathrm{~F} \mathrm{NMR}\left(376 \mathrm{MHz}, \mathrm{CDCl}_{3}\right): \delta-126.2--126.4(\mathrm{~m}),-150.4--$ $150.6(\mathrm{~m}),-160.7--160.9(\mathrm{~m}) .{ }^{13} \mathrm{C}$ NMR $\left(101 \mathrm{MHz}, \mathrm{CDCl}_{3}\right): \delta 149.0\left(\mathrm{dm}, \mathrm{C}-\mathrm{F},{ }^{1} \mathrm{~J}_{\mathrm{C}-\mathrm{F}}=\right.$ $243.1 \mathrm{~Hz}), 142.6\left(\mathrm{dm}, \mathrm{C}-\mathrm{F},{ }^{1} \mathrm{~J}_{\mathrm{C}-\mathrm{F}}=256.5 \mathrm{~Hz}\right), 137.4\left(\mathrm{dm}, \mathrm{C}-\mathrm{F},{ }^{1} \mathrm{~J}_{\mathrm{C}-\mathrm{F}}=254.9 \mathrm{~Hz}\right), 134.6$, 132.3, 130.5, 128.4, $108.2-107.5(\mathrm{~m}),-5.3\left(\mathrm{t}, \mathrm{C}-\mathrm{F},{ }^{4} \mathrm{~J}_{\mathrm{C}-\mathrm{F}}=3.2 \mathrm{~Hz}\right.$ ). IR (thin film): 2966 , 2173, 1462, 1084, 970, 822, 721. HRMS (El-TOF) calcd. for $\left[\mathrm{C}_{13} \mathrm{H}_{9} \mathrm{~F}_{5} \mathrm{Si}\right]\left([\mathrm{M}]^{+}\right)$: 288.0394 , found: 288.0392 .<smiles>COc1ccccc1[Si](C)(c1ccccc1)c1c(F)c(F)c(F)c(F)c1F</smiles>

(2-Methoxyphenyl)(methyl)(perfluorophenyl)silane (2b). 2-Methoxybromobenzene $(0.62 \mathrm{~mL}, 5.0 \mathrm{mmol})$ and bromopentafluorobenzene $(0.62 \mathrm{~mL}, 5.0 \mathrm{mmol})$ was subjected to method $A$ to give the corresponding hydrosilane as a colorless liquid $(1.0 \mathrm{~g}, 64 \%)$. $R_{f}=0.9$ (petroleum ether). ${ }^{1} \mathrm{H}$ NMR $\left(400 \mathrm{MHz}, \mathrm{CDCl}_{3}\right): \delta 7.49(\mathrm{t}, J=8.0 \mathrm{~Hz}, 2 \mathrm{H}), 7.05(\mathrm{t}, J=8.0$ $\mathrm{Hz}, 1 \mathrm{H}), 6.90(\mathrm{~d}, J=8.0 \mathrm{~Hz}, 1 \mathrm{H}), 5.20-5.16(\mathrm{~m}, 1 \mathrm{H}), 3.84(\mathrm{~s}, 3 \mathrm{H}), 0.82(\mathrm{~d}, J=4.0 \mathrm{~Hz}$, 3H). ${ }^{19} \mathrm{~F}$ NMR (376 MHz, $\left.\mathrm{CDCl}_{3}\right): \delta-126.8--126.9(\mathrm{~m}),-151.5--151.6(\mathrm{~m}),-161.5--$ $161.7(\mathrm{~m}) .{ }^{13} \mathrm{C}$ NMR $\left(101 \mathrm{MHz}, \mathrm{CDCl}_{3}\right):$ ס 164.4, $149.2\left(\mathrm{dm}, \mathrm{C}-\mathrm{F},{ }^{1} \mathrm{~J}_{\mathrm{C}-\mathrm{F}}=242.4 \mathrm{~Hz}\right), 142.4$ $\left(\mathrm{dm}, \mathrm{C}-\mathrm{F},{ }^{1} \mathrm{~J}_{\mathrm{C}-\mathrm{F}}=256.0 \mathrm{~Hz}\right), 137.3\left(\mathrm{dm}, \mathrm{C}-\mathrm{F},{ }^{1} \mathrm{~J}_{\mathrm{C}-\mathrm{F}}=254.0 \mathrm{~Hz}\right), 136.1,132.6,121.0,120.7$, 109.8, $108.7-108.0(\mathrm{~m}), 55.2,-5.2\left(\mathrm{t}, \mathrm{C}-\mathrm{F},{ }^{4} \mathrm{~J}_{\mathrm{C}-\mathrm{F}}=3.2 \mathrm{~Hz}\right.$ ). IR (thin film): 2955, 2173, 1463, 1242, 1083, 969, 900, 755. HRMS (EI-TOF) calcd. for [ $\left.\mathrm{C}_{14} \mathrm{H}_{11} \mathrm{~F}_{5} \mathrm{OSi}\right]\left([\mathrm{M}]^{+}\right): 318.0499$, found: 318.0506 . 
<smiles>COc1cc(F)c([Si](C)c2c(F)c(F)c(F)c(F)c2F)c(F)c1F</smiles>

(2,6-Difluoro-4-methoxyphenyl)(methyl)(perfluorophenyl)silane (2c). 4-Bromo-3,5difluoroanisole $(1.1 \mathrm{~g}, 5.0 \mathrm{mmol})$ and bromopentafluorobenzene $(0.62 \mathrm{~mL}, 5.0 \mathrm{mmol})$ was subjected to method $\mathrm{A}$ to give the corresponding hydrosilane as a colorless liquid $(0.65 \mathrm{~g}$, 37\%). $R_{f}=0.9$ (petroleum ether). ${ }^{1} \mathrm{H}$ NMR $\left(400 \mathrm{MHz}, \mathrm{CDCl}_{3}\right): \delta 6.41(\mathrm{~d}, J=8.0 \mathrm{~Hz}, 2 \mathrm{H})$, $5.23-5.18(\mathrm{~m}, 1 \mathrm{H}), 3.80(\mathrm{~s}, 3 \mathrm{H}), 0.78(\mathrm{~d}, J=4.0 \mathrm{~Hz}, 3 \mathrm{H}) .{ }^{19} \mathrm{~F} \mathrm{NMR}\left(376 \mathrm{MHz}, \mathrm{CDCl}_{3}\right): \delta$ $-96.9--96.9(\mathrm{~m}),-127.1--127.2(\mathrm{~m}),-150.6--150.7(\mathrm{~m}),-161.2--161.3(\mathrm{~m}) .{ }^{13} \mathrm{C}$ $\operatorname{NMR}\left(101 \mathrm{MHz}, \mathrm{CDCl}_{3}\right): \delta 169.0\left(\mathrm{~d}, \mathrm{C}-\mathrm{F},{ }^{2} \mathrm{~J}_{\mathrm{C}-\mathrm{F}}=17.8 \mathrm{~Hz}\right), 166.5\left(\mathrm{~d}, \mathrm{C}-\mathrm{F},{ }^{2} \mathrm{~J}_{\mathrm{C}-\mathrm{F}}=17.8 \mathrm{~Hz}\right)$, $164.3\left(\mathrm{t}, \mathrm{C}-\mathrm{F},{ }^{2} \mathrm{~J}_{\mathrm{C}-\mathrm{F}}=14.7 \mathrm{~Hz}\right), 149.0\left(\mathrm{dm}, \mathrm{C}-\mathrm{F},{ }^{1} \mathrm{~J}_{\mathrm{C}-\mathrm{F}}=245.0 \mathrm{~Hz}\right), 142.5\left(\mathrm{dm}, \mathrm{C}-\mathrm{F},{ }^{1} \mathrm{~J}_{\mathrm{C}-\mathrm{F}}\right.$ $=256.4 \mathrm{~Hz}), 137.1\left(\mathrm{dm}, \mathrm{C}-\mathrm{F},{ }^{1} \mathrm{~J}_{\mathrm{C}-\mathrm{F}}=254.5 \mathrm{~Hz}\right), 107.2-106.6(\mathrm{~m}), 97.9\left(\mathrm{dd}, \mathrm{C}-\mathrm{F},{ }^{2} \mathrm{~J}_{\mathrm{C}-\mathrm{F}}=\right.$ $\left.30.5 \mathrm{~Hz},{ }^{4} \mathrm{~J}_{\mathrm{C}-\mathrm{F}}=2.6 \mathrm{~Hz}\right), 55.7,-4.5--4.6(\mathrm{~m}) . \mathrm{IR}$ (thin film): $2181,1620,1464,1144,1106$, 823. HRMS (El-TOF) calcd. for [ $\left.\mathrm{C}_{14} \mathrm{H}_{9} \mathrm{~F}_{7} \mathrm{OSi}\right]\left([\mathrm{M}]^{+}\right)$: 354.0311, found: 354.0314 .<smiles>C[Si](c1cccs1)(c1cccs1)c1c(F)c(F)c(F)c(F)c1F</smiles>

Methyl(perfluorophenyl)(thiophen-2-yl)silane (2d). 2-Bromothiophene (0.49 mL, 5.0 $\mathrm{mmol})$ and bromopentafluorobenzene $(0.62 \mathrm{~mL}, 5.0 \mathrm{mmol})$ was subjected to method $A$ to give the corresponding hydrosilane as a colorless liquid $(0.30 \mathrm{~g}, 20 \%) . \mathrm{R}_{f}=0.9$ (petroleum ether). ${ }^{1} \mathrm{H}$ NMR $\left(400 \mathrm{MHz}, \mathrm{CDCl}_{3}\right): \delta 7.72(\mathrm{~d}, J=4.0 \mathrm{~Hz}, 1 \mathrm{H}), 7.48(\mathrm{~d}, J=4.0 \mathrm{~Hz}, 1 \mathrm{H}), 7.26$ $(\mathrm{t}, J=4.0 \mathrm{~Hz}, 1 \mathrm{H}), 5.35-5.30(\mathrm{~m}, 1 \mathrm{H}), 0.84(\mathrm{~d}, J=4.0 \mathrm{~Hz}, 3 \mathrm{H}) .{ }^{19} \mathrm{~F} \mathrm{NMR}\left(376 \mathrm{MHz}, \mathrm{CDCl}_{3}\right)$ : $\delta-126.4--126.6(\mathrm{~m}),-150.1--150.2(\mathrm{~m}),-160.6--160.8(\mathrm{~m}) .{ }^{13} \mathrm{C}$ NMR $(101 \mathrm{MHz}$, $\left.\mathrm{CDCl}_{3}\right): \delta 148.9\left(\mathrm{dm}, \mathrm{C}-\mathrm{F},{ }^{1} \mathrm{~J}_{\mathrm{C}-\mathrm{F}}==244.8 \mathrm{~Hz}\right), 142.7\left(\mathrm{dm}, \mathrm{C}-\mathrm{F},{ }^{1} \mathrm{~J}_{\mathrm{C}-\mathrm{F}}=256.9 \mathrm{~Hz}\right), 137.3$ (dm, C-F, $\left.{ }^{1} J_{\mathrm{C}-\mathrm{F}}=254.7 \mathrm{~Hz}\right), 137.2,132.7,130.2,128.6,107.7-107.0(\mathrm{~m}),-4.2(\mathrm{t}, \mathrm{C}-\mathrm{F}$, ${ }^{4} J_{C-F}=3.2 \mathrm{~Hz}$ ). IR (thin film): $21801515,1462,1084,970,820,714$. HRMS (El-TOF) calcd. for $\left[\mathrm{C}_{11} \mathrm{H}_{7} \mathrm{~F}_{5} \mathrm{SSi}\right]\left([\mathrm{M}]^{+}\right):$293.9958, found: 293.9946. 
<smiles>Cn1ccc2cc([Si](C)(C)c3c(F)c(F)c(F)c(F)c3F)ccc21</smiles>

1-Methyl-5-(methyl(perfluorophenyl)silyl)-1 H-indole (2e). 5-Bromo-1-methylindole (1.1 $\mathrm{g}, 5.00 \mathrm{mmol})$ and bromopentafluorobenzene $(0.62 \mathrm{~mL}, 5.0 \mathrm{mmol})$ was subjected to method $A$ to give the corresponding hydrosilane as a colorless liquid $(0.30 \mathrm{~g}, 18 \%)$. $R_{f}=$ 0.9 (petroleum ether). ${ }^{1} \mathrm{H} \mathrm{NMR}\left(400 \mathrm{MHz}, \mathrm{CDCl}_{3}\right): \delta 7.97$ (s, $\left.1 \mathrm{H}\right), 7.48(\mathrm{~d}, J=8.0 \mathrm{~Hz}, 1 \mathrm{H})$, $7.40(\mathrm{~d}, J=8.0 \mathrm{~Hz}, 1 \mathrm{H}), 7.10(\mathrm{~d}, J=4.0 \mathrm{~Hz}, 1 \mathrm{H}), 6.56(\mathrm{~d}, J=4.0 \mathrm{~Hz}, 1 \mathrm{H}), 5.31-5.26(\mathrm{~m}$, $1 \mathrm{H}), 3.82(\mathrm{~s}, 3 \mathrm{H}), 0.86(\mathrm{~d}, J=4.0 \mathrm{~Hz}, 3 \mathrm{H}) .{ }^{19} \mathrm{~F} \mathrm{NMR}\left(376 \mathrm{MHz}, \mathrm{CDCl}_{3}\right): \delta-126.4--126.5$ (m), $-151.2--151.3(\mathrm{~m}),-161.0--161.2(\mathrm{~m}) .{ }^{13} \mathrm{C}$ NMR (101 MHz, $\left.\mathrm{CDCl}_{3}\right)$ : $\delta 148.9(\mathrm{dm}$, $\left.\mathrm{C}-\mathrm{F},{ }^{1} \mathrm{~J}_{\mathrm{C}-\mathrm{F}}=243.7 \mathrm{~Hz}\right), 142.3\left(\mathrm{dm}, \mathrm{C}-\mathrm{F},{ }^{1} \mathrm{~J}_{\mathrm{C}-\mathrm{F}}=255.6 \mathrm{~Hz}\right), 137.9,137.3\left(\mathrm{dm}, \mathrm{C}-\mathrm{F},{ }^{1} \mathrm{~J}_{\mathrm{C}-\mathrm{F}}=\right.$ 254.7 Hz), 129.5, 128.7, 128.2, 126.9, 120.8, 109.6, 109.6 - $108.9(\mathrm{~m}), 101.3,32.9,-4.7$ (t, C-F, ${ }^{4} J_{C-F}=3.3 \mathrm{~Hz}$ ). IR (thin film): 2165, 1515, 1462, 1080, 969, 849. HRMS (El-TOF) calcd. for $\left[\mathrm{C}_{16} \mathrm{H}_{12} \mathrm{~F}_{5} \mathrm{NSi}\right]\left([\mathrm{M}]^{+}\right)$: 341.0659, found: 341.0652 .<smiles>COc1ccccc1[Si](C)(c1ccccc1)c1c(F)c(F)c(F)c(F)c1F</smiles>

Methyl(perfluorophenyl)(2-(trifluoromethoxy)phenyl)silane (2f). 2-Trifluoromethoxybromobenzene $(0.74 \mathrm{~mL}, 5.0 \mathrm{mmol})$ and bromopentafluorobenzene $(0.62 \mathrm{~mL}, 5.0 \mathrm{mmol})$ was subjected to method $A$ to give the corresponding hydrosilane as a colorless liquid ( 0.78 g, $42 \%) . \mathrm{R}_{f}=0.9$ (petroleum ether). ${ }^{1} \mathrm{H} \mathrm{NMR}\left(400 \mathrm{MHz}, \mathrm{CDCl}_{3}\right): \delta 7.59(\mathrm{~d}, J=8.0 \mathrm{~Hz}, 1 \mathrm{H})$, $7.52(\mathrm{~d}, J=8.0 \mathrm{~Hz}, 1 \mathrm{H}), 7.34(\mathrm{~d}, J=8.0 \mathrm{~Hz}, 1 \mathrm{H}), 7.29(\mathrm{~d}, J=8.0 \mathrm{~Hz}, 1 \mathrm{H}), 5.22(\mathrm{~s}, 1 \mathrm{H})$, $0.82(\mathrm{~d}, J=4.0 \mathrm{~Hz}, 3 \mathrm{H}) .{ }^{19} \mathrm{~F}$ NMR $\left(376 \mathrm{MHz}, \mathrm{CDCl}_{3}\right): \delta-56.9,-126.5--126.6(\mathrm{~m}),-150.1$ $--150.2(\mathrm{~m}),-160.9--161.1(\mathrm{~m}) .{ }^{13} \mathrm{C}$ NMR $\left(101 \mathrm{MHz}, \mathrm{CDCl}_{3}\right): \delta$ 154.4, $149.2(\mathrm{dm}, \mathrm{C}-\mathrm{F}$, $\left.{ }^{1} J_{\mathrm{C}-\mathrm{F}}=244.9 \mathrm{~Hz}\right), 142.8\left(\mathrm{dm}, \mathrm{C}-\mathrm{F},{ }^{1} \mathrm{~J}_{\mathrm{C}-\mathrm{F}}=256.7 \mathrm{~Hz}\right), 137.4\left(\mathrm{dm}, \mathrm{C}-\mathrm{F},{ }^{1} \mathrm{~J}_{\mathrm{C}-\mathrm{F}}=254.8 \mathrm{~Hz}\right)$, 136.6, 132.6, 126.6, 125.0, 120.5, 118.6, $107.1-106.5(\mathrm{~m}),-5.2\left(\mathrm{t}, \mathrm{C}-\mathrm{F},{ }^{4} \mathrm{~J}_{\mathrm{C}-\mathrm{F}}=3.1 \mathrm{~Hz}\right)$. IR (thin film): 2192, 1464, 1247, 1165, 1019, 970, 822. HRMS (El-TOF) calcd. for $\left[\mathrm{C}_{14} \mathrm{H}_{8} \mathrm{~F}_{8} \mathrm{OSi}\right]\left([\mathrm{M}]^{+}\right):$372.0217, found: 372.0213 . 
<smiles>C[Si](c1ccc(F)cc1)(c1ccc(F)cc1)c1c(F)c(F)c(F)c(F)c1F</smiles>

(4-Fluorophenyl)(methyl)(perfluorophenyl)silane (2g). 4-Bromofluorobenzene (1.1 mL, $10 \mathrm{mmol}$ ) and bromopentafluorobenzene $(0.62 \mathrm{~mL}, 5.0 \mathrm{mmol})$ was subjected to method $\mathrm{A}$ to give the corresponding hydrosilane as a colorless liquid $(0.38 \mathrm{~g}, 12 \%)$. $R_{f}=0.9$ (petroleum ether). ${ }^{1} \mathrm{H}$ NMR $\left(400 \mathrm{MHz}, \mathrm{CDCl}_{3}\right): \delta 7.66$ (t, $\left.J=4.0 \mathrm{~Hz}, 2 \mathrm{H}\right), 7.12(\mathrm{t}, J=8.0 \mathrm{~Hz}, 2 \mathrm{H})$, $5.24-5.19(\mathrm{~m}, 1 \mathrm{H}), 0.84(\mathrm{~d}, J=4.0 \mathrm{~Hz}, 3 \mathrm{H}) .{ }^{19} \mathrm{~F} \mathrm{NMR}\left(376 \mathrm{MHz}, \mathrm{CDCl}_{3}\right): \delta-109.5--$ $109.6(\mathrm{~m}),-126.6--126.7(\mathrm{~m}),-150.6--150.7(\mathrm{~m}),-160.9--161.1(\mathrm{~m}) .{ }^{13} \mathrm{C}$ NMR $(101$ $\left.\mathrm{MHz}, \mathrm{CDCl}_{3}\right): \delta 164.7\left(\mathrm{~d}, \mathrm{C}-\mathrm{F},{ }^{1} \mathrm{~J}_{\mathrm{C}-\mathrm{F}}=251.6 \mathrm{~Hz}\right), 149.2\left(\mathrm{dm}, \mathrm{C}-\mathrm{F},{ }^{1} \mathrm{~J}_{\mathrm{C}-\mathrm{F}}=244.0 \mathrm{~Hz}\right), 142.8$ $\left(\mathrm{dm}, \mathrm{C}-\mathrm{F},{ }^{1} \mathrm{~J}_{\mathrm{C}-\mathrm{F}}=256.3 \mathrm{~Hz}\right), 137.5\left(\mathrm{dm}, \mathrm{C}-\mathrm{F},{ }^{1} \mathrm{~J}_{\mathrm{C}-\mathrm{F}}=255.0 \mathrm{~Hz}\right), 136.8\left(\mathrm{~d}, \mathrm{C}-\mathrm{F},{ }^{3} \mathrm{~J}_{\mathrm{C}-\mathrm{F}}=7.8\right.$ $\mathrm{Hz}), 127.9\left(\mathrm{~d}, \mathrm{C}-\mathrm{F},{ }^{4} \mathrm{~J}_{\mathrm{C}-\mathrm{F}}=3.3 \mathrm{~Hz}\right), 115.7\left(\mathrm{~d}, \mathrm{C}-\mathrm{F},{ }^{2} \mathrm{~J}_{\mathrm{C}-\mathrm{F}}=20.2 \mathrm{~Hz}\right), 108.1-107.4(\mathrm{~m}),-$ $5.3\left(t, C-F,{ }^{4} J_{C-F}=3.1 \mathrm{~Hz}\right.$ ). IR (thin film): 2178, 1517, 1465, 1088, 972, 855. HRMS (EITOF) calcd. for $\left[\mathrm{C}_{13} \mathrm{H}_{8} \mathrm{~F}_{6} \mathrm{Si}\right]\left([\mathrm{M}]^{+}\right)$: 306.0299, found: 306.0301 .<smiles>Cc1ccc([Si](C)(C)c2c(F)c(F)c(F)c(F)c2F)cc1</smiles>

Methyl(perfluorophenyl)(p-tolyl)silane (2h). 4-Methylbromobenzene (0.62 mL, 5.0 $\mathrm{mmol})$ and bromopentafluorobenzene $(0.62 \mathrm{~mL}, 5.0 \mathrm{mmol})$ was subjected to method $A$ to give the corresponding hydrosilane as a colorless liquid ( $0.60 \mathrm{~g}, 40 \%) . \mathrm{R}_{f}=0.9$ (petroleum ether). ${ }^{1} \mathrm{H}$ NMR $\left(400 \mathrm{MHz}, \mathrm{CDCl}_{3}\right): \delta 7.56(\mathrm{~d}, J=4.0 \mathrm{~Hz}, 2 \mathrm{H}), 7.26(\mathrm{~d}, J=8.0 \mathrm{~Hz}, 2 \mathrm{H}), 5.20$ $-5.15(\mathrm{~m}, 1 \mathrm{H}), 2.41(\mathrm{~s}, 3 \mathrm{H}), 0.80(\mathrm{~d}, J=4.0 \mathrm{~Hz}, 3 \mathrm{H}) .{ }^{19} \mathrm{~F} \mathrm{NMR}\left(376 \mathrm{MHz}, \mathrm{CDCl}_{3}\right): \delta-126.4$ $--126.5(\mathrm{~m}),-150.7--150.9(\mathrm{~m}),-160.9--161.0(\mathrm{~m}) .{ }^{13} \mathrm{C} \mathrm{NMR}\left(101 \mathrm{MHz}, \mathrm{CDCl}_{3}\right): \delta$ $149.0\left(\mathrm{dm}, \mathrm{C}-\mathrm{F},{ }^{1} \mathrm{~J}_{\mathrm{C}-\mathrm{F}}=244.0 \mathrm{~Hz}\right), 142.6\left(\mathrm{dm}, \mathrm{C}-\mathrm{F},{ }^{1} \mathrm{~J}_{\mathrm{C}-\mathrm{F}}=256.3 \mathrm{~Hz}\right), 140.7,137.4(\mathrm{dm}$, $\left.\mathrm{C}-\mathrm{F},{ }^{1} \mathrm{~J}_{\mathrm{C}-\mathrm{F}}=254.8 \mathrm{~Hz}\right), 134.7,129.2,128.6,108.5-107.8(\mathrm{~m}), 21.6,-5.2\left(\mathrm{t}, \mathrm{C}-\mathrm{F},{ }^{4} \mathrm{~J}_{\mathrm{C}-\mathrm{F}}=\right.$ 3.3 Hz). IR (thin film): 2170, 1515, 1460, 1082, 969, 851, 745. HRMS (El-TOF) calcd. for $\left[\mathrm{C}_{14} \mathrm{H}_{11} \mathrm{~F}_{5} \mathrm{Si}\right]\left([\mathrm{M}]^{+}\right):$302.0550, found: 302.0552 . 
<smiles>COc1ccc([Si](C)(C)c2c(F)c(F)c(F)c(F)c2F)cc1</smiles>

(4-Methoxyphenyl)(methyl)(perfluorophenyl)silane (2i). 4-Methoxybromobenzene $(0.63 \mathrm{~mL}, 5.0 \mathrm{mmol})$ and bromopentafluorobenzene $(0.62 \mathrm{~mL}, 5.0 \mathrm{mmol})$ was subjected to method $A$ to give the corresponding hydrosilane as a colorless liquid $(0.57 \mathrm{~g}, 36 \%)$. $\mathrm{R}_{f}=$ 0.9 (petroleum ether). ${ }^{1} \mathrm{H} \mathrm{NMR}\left(400 \mathrm{MHz}, \mathrm{CDCl}_{3}\right): \delta 7.57$ (d, $\left.J=8.0 \mathrm{~Hz}, 2 \mathrm{H}\right), 6.96(\mathrm{~d}, J=$ $8.0 \mathrm{~Hz}, 2 \mathrm{H}), 5.18-5.13(\mathrm{~m}, 1 \mathrm{H}), 3.84(\mathrm{~s}, 3 \mathrm{H}), 0.78(\mathrm{~d}, J=4.0 \mathrm{~Hz}, 3 \mathrm{H}) .{ }^{19} \mathrm{~F}$ NMR $(376 \mathrm{MHz}$, $\left.\mathrm{CDCl}_{3}\right): \delta-126.6--126.7(\mathrm{~m}),-150.9--151.0(\mathrm{~m}),-161.0--161.1(\mathrm{~m}) .{ }^{13} \mathrm{C}$ NMR $(101$ $\left.\mathrm{MHz}, \mathrm{CDCl}_{3}\right): \delta 161.6,149.0\left(\mathrm{dm}, \mathrm{C}-\mathrm{F},{ }^{1} \mathrm{~J}_{\mathrm{C}-\mathrm{F}}=243.6 \mathrm{~Hz}\right), 142.5\left(\mathrm{dm}, \mathrm{C}-\mathrm{F},{ }^{1} \mathrm{~J}_{\mathrm{C}-\mathrm{F}}=256.0\right.$ $\mathrm{Hz}), 137.4\left(\mathrm{dm}, \mathrm{C}-\mathrm{F},{ }^{1} \mathrm{~J}_{\mathrm{C}-\mathrm{F}}=254.4 \mathrm{~Hz}\right), 136.2,122.8,114.2,108.6-108.0(\mathrm{~m}), 55.1,-5.1$ (t, C-F, ${ }^{4} J_{C-F}=3.1 \mathrm{~Hz}$ ). IR (thin film): 2169, 1460, 1282, 1082, 968, 817. HRMS (El-TOF) calcd. for $\left[\mathrm{C}_{14} \mathrm{H}_{11} \mathrm{~F}_{5} \mathrm{OSi}\right]\left([\mathrm{M}]^{+}\right): 318.0499$, found: 318.0506 .<smiles>CN(C)c1ccc([Si](C)(C)c2c(F)c(F)c(F)c(F)c2F)cc1</smiles>

$N, N$-Dimethyl-4-(methyl(perfluorophenyl)silyl)aniline (2j). 4-Bromo- $N, N$-dimethylaniline $(1.0 \mathrm{~g}, 5.0 \mathrm{mmol})$ and bromopentafluorobenzene $(0.62 \mathrm{~mL}, 5.0 \mathrm{mmol})$ was subjected to method $A$ to give the corresponding hydrosilane as a colorless liquid $(0.73 \mathrm{~g}, 44 \%)$. $R_{f}=$ 0.9 (petroleum ether). ${ }^{1} \mathrm{H}$ NMR $\left(400 \mathrm{MHz}, \mathrm{CDCl}_{3}\right): \delta 7.54(\mathrm{~d}, J=12.0 \mathrm{~Hz}, 2 \mathrm{H}), 6.78$ (d, $J=$ $8.0 \mathrm{~Hz}, 2 \mathrm{H}), 5.19-5.14(\mathrm{~m}, 1 \mathrm{H}), 3.04(\mathrm{~s}, 6 \mathrm{H}), 0.78(\mathrm{~d}, J=4.0 \mathrm{~Hz}, 3 \mathrm{H}) .{ }^{19} \mathrm{~F} \mathrm{NMR}(376 \mathrm{MHz}$, $\left.\mathrm{CDCl}_{3}\right): \delta-126.6--126.7(\mathrm{~m}),-151.4--151.6(\mathrm{~m}),-161.1--161.3(\mathrm{~m}) .{ }^{13} \mathrm{C}$ NMR $(101$ $\left.\mathrm{MHz}, \mathrm{CDCl}_{3}\right): \delta 151.8,148.8\left(\mathrm{dm}, \mathrm{C}-\mathrm{F},{ }^{1} \mathrm{~J}_{\mathrm{C}-\mathrm{F}}=243.1 \mathrm{~Hz}\right), 142.2\left(\mathrm{dm}, \mathrm{C}-\mathrm{F},{ }^{1} \mathrm{~J}_{\mathrm{C}-\mathrm{F}}=255.4\right.$ $\mathrm{Hz}), 137.1\left(\mathrm{dm}, \mathrm{C}-\mathrm{F},{ }^{1} \mathrm{~J}_{\mathrm{C}-\mathrm{F}}=254.7 \mathrm{~Hz}\right), 135.7,116.2,111.9,109.2-108.5(\mathrm{~m}), 40.0,-5.0$ (t, C-F, ${ }^{4} J_{C-F}=3.3 \mathrm{~Hz}$ ). IR (thin film): 2165, 1642, 1543, 1458, 1080, 884, 806. HRMS (EITOF) calcd. for $\left[\mathrm{C}_{15} \mathrm{H}_{14} \mathrm{~F}_{5} \mathrm{NSi}\right]\left([\mathrm{M}]^{+}\right): 331.0816$, found: 331.0810 .<smiles>C[Si](Cc1ccccc1)c1c(F)c(F)c(F)c(F)c1F</smiles> 
Benzyl(methyl)(perfluorophenyl)silane (2k). Benzyl bromide $(0.59 \mathrm{~mL}, 5.0 \mathrm{mmol})$ and bromopentafluorobenzene $(0.62 \mathrm{~mL}, 5.0 \mathrm{mmol})$ was subjected to method $A$ to give the corresponding hydrosilane as a colorless liquid $(0.21 \mathrm{~g}, 14 \%) . \mathrm{R}_{f}=0.9$ (petroleum ether). ${ }^{1} \mathrm{H} \mathrm{NMR}\left(400 \mathrm{MHz}, \mathrm{CDCl}_{3}\right): \delta 7.27(\mathrm{t}, J=8.0 \mathrm{~Hz}, 2 \mathrm{H}), 7.16(\mathrm{t}, J=8.0 \mathrm{~Hz}, 1 \mathrm{H}), 7.08(\mathrm{~d}, J=$ $4.0 \mathrm{~Hz}, 2 \mathrm{H}), 4.70-4.65(\mathrm{~m}, 1 \mathrm{H}), 2.58-2.48(\mathrm{~m}, 2 \mathrm{H}), 0.48(\mathrm{~d}, J=4.0 \mathrm{~Hz}, 3 \mathrm{H}) .{ }^{19} \mathrm{~F} \mathrm{NMR}$ $\left(376 \mathrm{MHz}, \mathrm{CDCl}_{3}\right): \delta-126.8,-150.6,-161.0 .{ }^{13} \mathrm{C} \mathrm{NMR}\left(101 \mathrm{MHz}, \mathrm{CDCl}_{3}\right): \delta 149.0(\mathrm{dm}$, $\left.\mathrm{C}-\mathrm{F},{ }^{1} \mathrm{~J}_{\mathrm{C}-\mathrm{F}}=243.4 \mathrm{~Hz}\right), 142.5\left(\mathrm{dm}, \mathrm{C}-\mathrm{F},{ }^{1} \mathrm{~J}_{\mathrm{C}-\mathrm{F}}=256.3 \mathrm{~Hz}\right), 137.7,137.3\left(\mathrm{dm}, \mathrm{C}-\mathrm{F},{ }^{1} \mathrm{~J}_{\mathrm{C}-\mathrm{F}}=\right.$ $254.9 \mathrm{~Hz}), 128.7,128.3,125.2,107.8-107.1(\mathrm{~m}), 22.6,-5.8\left(\mathrm{t}, \mathrm{C}-\mathrm{F},{ }^{4} J_{\mathrm{C}-\mathrm{F}}=2.7 \mathrm{~Hz}\right) . \mathrm{IR}$ (thin film): $2172,1514,1494,1082,968,856,697$. HRMS (El-TOF) calcd. for $\left[\mathrm{C}_{14} \mathrm{H}_{11} \mathrm{~F}_{5} \mathrm{Si}\right]$ $\left([\mathrm{M}]^{+}\right): 302.0550$, found: 302.0544 .<smiles>C[Si](CCc1ccccc1)c1c(F)c(F)c(F)c(F)c1F</smiles>

Methyl(perfluorophenyl)(phenethyl)silane (2I). (2-Bromoethyl)benzene (0.68 mL, 5.0 $\mathrm{mmol})$ and bromopentafluorobenzene $(0.62 \mathrm{~mL}, 5.0 \mathrm{mmol})$ was subjected to method A to give the corresponding hydrosilane as a colorless liquid $(0.98 \mathrm{~g}, 62 \%) . \mathrm{R}_{f}=0.9$ (petroleum ether). ${ }^{1} \mathrm{H}$ NMR (400 MHz, $\mathrm{CDCl}_{3}$ ): $\delta 7.29$ (t, $\left.J=8.0 \mathrm{~Hz}, 2 \mathrm{H}\right), 7.19$ (t, $\left.J=8.0 \mathrm{~Hz}, 3 \mathrm{H}\right), 4.64$ $-4.60(\mathrm{~m}, 1 \mathrm{H}), 2.84-2.71(\mathrm{~m}, 2 \mathrm{H}), 1.40-1.33(\mathrm{~m}, 2 \mathrm{H}), 0.48(\mathrm{~d}, J=4.0 \mathrm{~Hz}, 3 \mathrm{H}) .{ }^{19} \mathrm{~F}$ NMR $\left(376 \mathrm{MHz}, \mathrm{CDCl}_{3}\right): \delta-126.8--126.9(\mathrm{~m}),-151.0--151.1(\mathrm{~m}),-161.0--161.2(\mathrm{~m}) .{ }^{13} \mathrm{C}$ $\operatorname{NMR}(101 \mathrm{MHz}, \mathrm{CDCl}): \delta 148.9\left(\mathrm{dm}, \mathrm{C}-\mathrm{F},{ }^{1} \mathrm{~J}_{\mathrm{C}-\mathrm{F}}=242.9 \mathrm{~Hz}\right), 143.4,142.3\left(\mathrm{dm}, \mathrm{C}-\mathrm{F},{ }^{1} \mathrm{JC}_{\mathrm{C}}\right.$ $\mathrm{F}=256.0 \mathrm{~Hz}), 137.2\left(\mathrm{dm}, \mathrm{C}-\mathrm{F},{ }^{1} \mathrm{~J}_{\mathrm{C}-\mathrm{F}}=254.7 \mathrm{~Hz}\right), 128.5,128.0,126.1,108.2-107.5(\mathrm{~m})$, $30.4,14.9,-5.4$ (t, C-F, ${ }^{4} J_{C-F}=2.6 \mathrm{~Hz}$ ). IR (thin film): 2164, 1459, 1082, 968, 818, 698. HRMS (El-TOF) calcd. for [ $\left.\mathrm{C}_{15} \mathrm{H}_{13} \mathrm{~F}_{5} \mathrm{Si}\right]\left([\mathrm{M}]^{+}\right)$: 316.0707, found: 316.0703.<smiles>CCC[Si](C)(C)c1c(F)c(F)c(F)c(F)c1F</smiles>

Methyl(perfluorophenyl)(propyl)silane (2m). 1-Bromopropane $(0.45 \mathrm{~mL}, 5.0 \mathrm{mmol})$ and bromopentafluorobenzene $(0.62 \mathrm{~mL}, 5.0 \mathrm{mmol})$ was subjected to method $A$ to give the corresponding hydrosilane as a colorless liquid $(0.78 \mathrm{~g}, 61 \%) . \mathrm{R}_{f}=0.9$ (petroleum ether). 
${ }^{1} \mathrm{H}$ NMR $\left(400 \mathrm{MHz}, \mathrm{CDCl}_{3}\right): \delta 4.56-4.51(\mathrm{~m}, 1 \mathrm{H}), 1.45-1.38(\mathrm{~m}, 2 \mathrm{H}), 0.98(\mathrm{t}, J=8.0 \mathrm{~Hz}$, $3 \mathrm{H}), 0.95-0.90(\mathrm{~m}, 2 \mathrm{H}), 0.42(\mathrm{~d}, J=4.0 \mathrm{~Hz}, 3 \mathrm{H}) .{ }^{19} \mathrm{~F} \mathrm{NMR}\left(376 \mathrm{MHz}, \mathrm{CDCl}_{3}\right): \delta-127.0-$ $-127.1(\mathrm{~m}),-151.2--151.3(\mathrm{~m}),-161.2--161.4(\mathrm{~m}) .{ }^{13} \mathrm{C}$ NMR $\left(101 \mathrm{MHz}, \mathrm{CDCl}_{3}\right): \delta$ $148.8\left(\mathrm{dm}, \mathrm{C}-\mathrm{F},{ }^{1} \mathrm{~J}_{\mathrm{C}-\mathrm{F}}=243.2 \mathrm{~Hz}\right), 142.1\left(\mathrm{dm}, \mathrm{C}-\mathrm{F},{ }^{1} \mathrm{~J}_{\mathrm{C}-\mathrm{F}}=255.8 \mathrm{~Hz}\right), 137.1(\mathrm{dm}, \mathrm{C}-\mathrm{F}$, $\left.{ }^{1} J_{C-F}=254.6 \mathrm{~Hz}\right), 108.4-107.7(\mathrm{~m}), 17.7,17.4,15.2\left(\mathrm{t}, \mathrm{C}-\mathrm{F},{ }^{4} J_{\mathrm{C}-\mathrm{F}}=1.5 \mathrm{~Hz}\right),-5.6(\mathrm{t}, \mathrm{C}-$ $F,{ }^{4} J_{C-F}=2.8 \mathrm{~Hz}$ ). IR (thin film): 2963, 2137, 1501, 1461, 1084, 965, 883. HRMS (El-TOF) calcd. for $\left[\mathrm{C}_{10} \mathrm{H}_{11} \mathrm{~F}_{5} \mathrm{Si}\right]\left([\mathrm{M}]^{+}\right):$254.0550, found: 254.0555 .<smiles>C[Si](C)(C)c1c(F)c(F)c(F)c(F)c1F</smiles>

Dimethyl(perfluorophenyl)silane (2n). Bromopentafluorobenzene (1.3 mL, $10 \mathrm{mmol})$ was subjected to method $B$ to give the corresponding hydrosilane as a colorless liquid (1.7 g, 74\%). $\mathrm{R}_{f}=0.9$ (petroleum ether). ${ }^{1} \mathrm{H} \mathrm{NMR}\left(400 \mathrm{MHz}, \mathrm{CDCl}_{3}\right): \delta 4.65-4.59(\mathrm{~m}, 1 \mathrm{H})$, $0.46(\mathrm{~d}, J=4.0 \mathrm{~Hz}, 6 \mathrm{H}) .{ }^{19} \mathrm{~F} \mathrm{NMR}\left(376 \mathrm{MHz}, \mathrm{CDCl}_{3}\right): \delta-127.7--127.8(\mathrm{~m}),-151.6--$ $151.8(\mathrm{~m}),-161.5--161.7(\mathrm{~m}) \cdot{ }^{13} \mathrm{C}$ NMR $\left(101 \mathrm{MHz}, \mathrm{CDCl}_{3}\right): \delta 148.8\left(\mathrm{dm}, \mathrm{C}-\mathrm{F},{ }^{1} \mathrm{~J} \mathrm{C}-\mathrm{F}=\right.$ 243.3 Hz), 142.1 (dm, C-F, $\left.{ }^{1} J_{C-F}=255.7 \mathrm{~Hz}\right), 137.1\left(\mathrm{dm}, \mathrm{C}-\mathrm{F},{ }^{1} \mathrm{~J}_{\mathrm{C}-\mathrm{F}}=254.5 \mathrm{~Hz}\right), 109.0-$ $108.3(\mathrm{~m}),-4.0\left(\mathrm{t}, \mathrm{C}-\mathrm{F},{ }^{4} \mathrm{~J}_{\mathrm{C}-\mathrm{F}}=2.0 \mathrm{~Hz}\right)$. IR (thin film): 2170, 1460, 1080, 969, 884, 777. HRMS (El-TOF) calcd. for $\left[\mathrm{C}_{8} \mathrm{H}_{7} \mathrm{~F}_{5} \mathrm{Si}\right]\left([\mathrm{M}]^{+}\right): 226.0237$, found: 226.0238.

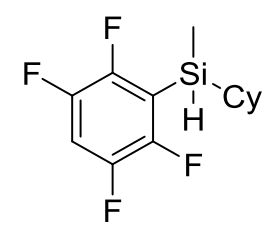

Cyclohexyl(methyl)(2,3,5,6-tetrafluorophenyl)silane (20). Bromocyclohexane (1.2 mL, $10 \mathrm{mmol}$ ) and bromopentafluorobenzene $(1.2 \mathrm{~mL}, 10 \mathrm{mmol})$ was subjected to method $\mathrm{A}$ to give the corresponding hydrosilane as a colorless liquid $(0.42 \mathrm{~g}, 15 \%) . \mathrm{R}_{f}=0.9$ (petroleum ether). ${ }^{1} \mathrm{H}$ NMR (400 MHz, $\mathrm{CDCl}_{3}$ ): $\delta 7.05$ (ddd, $\left.J=16.8,9.3,7.4 \mathrm{~Hz}, 1 \mathrm{H}\right), 4.42-4.34(\mathrm{~m}$, $1 \mathrm{H}), 1.81-1.64(\mathrm{~m}, 5 \mathrm{H}), 1.30-1.13(\mathrm{~m}, 5 \mathrm{H}), 1.11-1.03(\mathrm{~m}, 1 \mathrm{H}), 0.41(\mathrm{~d}, J=3.9 \mathrm{~Hz}, 3 \mathrm{H})$. ${ }^{19} \mathrm{~F}$ NMR $\left(376 \mathrm{MHz}, \mathrm{CDCl}_{3}\right): \delta-127.2--127.4(\mathrm{~m}),-138.8--139.0(\mathrm{~m}) .{ }^{13} \mathrm{C}$ NMR $(101$ $\left.\mathrm{MHz}, \mathrm{CDCl}_{3}\right): \delta 148.8\left(\mathrm{dtd}, \mathrm{C}-\mathrm{F},{ }^{1} J_{\mathrm{C}-\mathrm{F}}=242.2 \mathrm{~Hz},{ }^{2} J_{\mathrm{C}-\mathrm{F}}=12.7 \mathrm{~Hz},{ }^{3} J_{\mathrm{C}-\mathrm{F}}=3.6 \mathrm{~Hz}\right), 145.8$ (dddd, C-F, ${ }^{1} J_{C-F}=251.2 \mathrm{~Hz},{ }^{2} J_{C-F}=18.3 \mathrm{~Hz},{ }^{3} J_{C-F}=8.1 \mathrm{~Hz},{ }^{4} J_{C-F}=3.5 \mathrm{~Hz}$ ), 114.6 (t, C- 
$\left.\mathrm{F},{ }^{2} J_{\mathrm{C}-\mathrm{F}}=32.8 \mathrm{~Hz}\right), 107.9\left(\mathrm{tt}, \mathrm{C}-\mathrm{F},{ }^{2} \mathrm{~J}_{\mathrm{C}-\mathrm{F}}=22.7 \mathrm{~Hz},{ }^{4} \mathrm{~J}_{\mathrm{C}-\mathrm{F}}=1.8 \mathrm{~Hz}\right), 28.1,27.9,27.7,27.7$, 26.7, 23.7, -7.4 (t, C-F, $\left.{ }^{4} \mathrm{~J}_{\mathrm{C}-\mathrm{F}}=3.4 \mathrm{~Hz}\right)$. HRMS (El-TOF) calcd. for $\left[\mathrm{C}_{13} \mathrm{H}_{16} \mathrm{~F}_{4} \mathrm{Si}\right]\left([\mathrm{M}]^{+}\right)$: 276.0957, found: 276.0954 .<smiles>C[Si](C)c1c(F)c(F)cc(F)c1F</smiles>

Dimethyl(2,3,5,6-tetrafluorophenyl)silane (2p). 1-Bromo-2,3,5,6-tetrafluorobenzene $(1.2 \mathrm{~mL}, 10 \mathrm{mmol})$ was subjected to method $B$ to give the corresponding hydrosilane as a colorless liquid (1.6 g, 77\%). $\mathrm{R}_{f}=0.9$ (petroleum ether). ${ }^{1} \mathrm{H} \mathrm{NMR}\left(400 \mathrm{MHz}, \mathrm{CDCl}_{3}\right): \delta 7.09$ $-7.00(\mathrm{~m}, 1 \mathrm{H}), 4.66-4.60(\mathrm{~m}, 1 \mathrm{H}), 0.46(\mathrm{~d}, J=4.0 \mathrm{~Hz}, 6 \mathrm{H}) .{ }^{19} \mathrm{~F} \mathrm{NMR}\left(376 \mathrm{MHz}, \mathrm{CDCl}_{3}\right)$ : $\delta-128.8--128.9(\mathrm{~m}),-139.1--139.2(\mathrm{~m}) .{ }^{13} \mathrm{C}$ NMR $\left(101 \mathrm{MHz}, \mathrm{CDCl}_{3}\right): \delta 148.9(\mathrm{dm}, \mathrm{C}-$ $\left.\mathrm{F},{ }^{1} \mathrm{~J}_{\mathrm{C}-\mathrm{F}}=242.6 \mathrm{~Hz}\right), 145.8\left(\mathrm{dm}, \mathrm{C}-\mathrm{F},{ }^{1} \mathrm{~J}_{\mathrm{C}-\mathrm{F}}=251.5 \mathrm{~Hz}\right), 116.1-115.5(\mathrm{~m}), 107.8(\mathrm{t}, \mathrm{C}-\mathrm{F}$, ${ }^{2} J_{C-F}=22.7 \mathrm{~Hz}$ ), $-3.8\left(\mathrm{~d}, \mathrm{C}-\mathrm{F},{ }^{4} \mathrm{~J}_{\mathrm{C}-\mathrm{F}}=1.9 \mathrm{~Hz}\right)$. IR (thin film): 2168, 1464, 1229, 1168, 889, 835, 777. HRMS (EI-TOF) calcd. for $\left[\mathrm{C}_{8} \mathrm{H}_{8} \mathrm{~F}_{4} \mathrm{Si}\right]\left([\mathrm{M}]^{+}\right)$: 208.0331, found: 208.0327.<smiles>COc1c(F)c(F)c([Si](C)(C)C)c(F)c1F</smiles>

Dimethyl(2,3,5,6-tetrafluoro-4-methoxyphenyl)silane (2q). 1-Bromo-2,3,5,6-tetrafluoro-4-methoxybenzene $(1.0 \mathrm{~g}, 3.9 \mathrm{mmol})$ was subjected to method $\mathrm{B}$ to give the corresponding hydrosilane as a colorless liquid $(0.57 \mathrm{~g}, 61 \%) . \mathrm{R}_{f}=0.9$ (petroleum ether). ${ }^{1} \mathrm{H}$ $\operatorname{NMR}\left(400 \mathrm{MHz}, \mathrm{CDCl}_{3}\right): \delta 4.63-4.55(\mathrm{~m}, 1 \mathrm{H}), 4.09-4.08(\mathrm{~m}, 3 \mathrm{H}), 0.43(\mathrm{~d}, J=4.0 \mathrm{~Hz}$, 6H). ${ }^{19} \mathrm{~F} \mathrm{NMR}\left(376 \mathrm{MHz}, \mathrm{CDCl}_{3}\right): \delta-129.3--129.4(\mathrm{~m}),-157.5--157.6(\mathrm{~m}) .{ }^{13} \mathrm{C} \mathrm{NMR}$ $\left(101 \mathrm{MHz}, \mathrm{CDCl}_{3}\right): \delta 149.2\left(\mathrm{dm}, \mathrm{C}-\mathrm{F},{ }^{1} \mathrm{~J}_{\mathrm{C}-\mathrm{F}}=241.4 \mathrm{~Hz}\right), 140.3\left(\mathrm{dm}, \mathrm{C}-\mathrm{F},{ }^{1} \mathrm{~J}_{\mathrm{C}-\mathrm{F}}=252.8 \mathrm{~Hz}\right)$, $139.8-139.0(\mathrm{~m}), 106.5\left(\mathrm{t}, \mathrm{C}-\mathrm{F},{ }^{2} \mathrm{~J}_{\mathrm{C}-\mathrm{F}}=32.9 \mathrm{~Hz}\right), 61.9$ (t, C-F, $\left.{ }^{3} \mathrm{~J}_{\mathrm{C}-\mathrm{F}}=3.9 \mathrm{~Hz}\right),-3.6$ (t, C$\mathrm{F},{ }^{4} \mathrm{~J}_{\mathrm{C}-\mathrm{F}}=2.4 \mathrm{~Hz}$ ). HRMS (El-TOF) calcd. for $\left[\mathrm{C}_{9} \mathrm{H}_{10} \mathrm{~F}_{4} \mathrm{OSi}\right]\left([\mathrm{M}]^{+}\right): 238.0437$, found: 238.0430 .<smiles>C[SiH](C)c1c(F)cc(F)cc1F</smiles> 
Dimethyl(2,4,6-trifluorophenyl)silane (2r). 2-Bromo-1,3,5-trifluorobenzene $(0.29 \mathrm{~mL}$, $2.42 \mathrm{mmol}$ ) was subjected to method $B$ to give the corresponding hydrosilane as a colorless liquid $(0.24 \mathrm{~g}, 52 \%) . \mathrm{R}_{f}=0.9$ (petroleum ether). ${ }^{1} \mathrm{H}$ NMR (400 MHz, Chloroform-d) $\delta 6.59(\mathrm{t}, J=8.0 \mathrm{~Hz}, 2 \mathrm{H}), 4.61-4.51(\mathrm{~m}, 1 \mathrm{H}), 0.40(\mathrm{~d}, J=3.8 \mathrm{~Hz}, 6 \mathrm{H}) .{ }^{19} \mathrm{~F}$ NMR (376 MHz, $\left.\mathrm{CDCl}_{3}\right) \delta-95.33--95.43(\mathrm{~m}),-106.22--106.37(\mathrm{~m}) .{ }^{13} \mathrm{C}$ NMR $(101$ $\mathrm{MHz}, \mathrm{CDCl}_{3}$ ): $\delta 167.5$ (ddd, C-F, ${ }^{1} \mathrm{~J}_{\mathrm{C}-\mathrm{F}}=245.4 \mathrm{~Hz},{ }^{2} \mathrm{~J}_{\mathrm{C}-\mathrm{F}}=19.1 \mathrm{~Hz},{ }^{2} J_{\mathrm{C}-\mathrm{F}}=14.9 \mathrm{~Hz}$ ), 164.8 (dt, C-F, ${ }^{1} J_{C-F}=251.3 \mathrm{~Hz},{ }^{2} J_{\mathrm{C}-\mathrm{F}}=15.9 \mathrm{~Hz}$ ), 107.5 (dt, C-F, ${ }^{2} \mathrm{~J}_{\mathrm{C}-\mathrm{F}}=36.9 \mathrm{~Hz},{ }^{3} \mathrm{~J}_{\mathrm{C}-\mathrm{F}}$ $=4.1 \mathrm{~Hz}$ ), 100.0 (ddd, C-F, $\left.{ }^{2} \mathrm{~J}_{\mathrm{C}-\mathrm{F}}=31.7 \mathrm{~Hz},{ }^{2} \mathrm{~J}_{\mathrm{C}-\mathrm{F}}=24.6 \mathrm{~Hz},{ }^{3} \mathrm{~J}_{\mathrm{C}-\mathrm{F}}=4.1 \mathrm{~Hz}\right),-3.38$ (t, C$\mathrm{F},{ }^{4} \mathrm{~J}_{\mathrm{C}-\mathrm{F}}=1.8 \mathrm{~Hz}$ ). HRMS (El-TOF) calcd. for $\left[\mathrm{C}_{8} \mathrm{H}_{9} \mathrm{~F}_{3} \mathrm{Si}\right]\left([\mathrm{M}]^{+}\right): 190.0426$, found: 190.0427 .<smiles>CC(C)[Si](c1c(F)cc(F)cc1F)(C(C)C)C(F)(F)F</smiles>

Diisopropyl(2,4,6-trifluorophenyl)silane (2s). 2-Bromo-1,3,5-trifluorobenzene (1.2 mL, $10 \mathrm{mmol}$ ) was subjected to method $B$ to give the corresponding hydrosilane as a colorless liquid (1.6 g, 65\%). $\mathrm{R}_{f}=0.9$ (petroleum ether). ${ }^{1} \mathrm{H} \mathrm{NMR}\left(400 \mathrm{MHz}, \mathrm{CDCl}_{3}\right): \delta 6.67-6.61$ (m, 2H), $4.14-4.09(\mathrm{~m}, 1 \mathrm{H}), 1.40-1.32(\mathrm{~m}, 2 \mathrm{H}), 1.10(\mathrm{~d}, J=7.6 \mathrm{~Hz}, 6 \mathrm{H}), 1.01$ (d, J = 7.6 $\mathrm{Hz}, 6 \mathrm{H}) .{ }^{19} \mathrm{~F} \mathrm{NMR}\left(376 \mathrm{MHz}, \mathrm{CDCl}_{3}\right): \delta-93.0--93.1(\mathrm{~m}),-106.1--106.2(\mathrm{~m}) .{ }^{13} \mathrm{C} \mathrm{NMR}$ $\left(101 \mathrm{MHz}, \mathrm{CDCl}_{3}\right): \delta 167.3\left(\mathrm{ddd}, \mathrm{C}-\mathrm{F},{ }^{1} \mathrm{~J}_{\mathrm{C}-\mathrm{F}}=245.4 \mathrm{~Hz},{ }^{2} J_{\mathrm{C}-\mathrm{F}}=19.1 \mathrm{~Hz},{ }^{2} J_{\mathrm{C}-\mathrm{F}}=14.9 \mathrm{~Hz}\right.$ ), 164.7 (dt, C-F, ${ }^{1} \mathrm{~J}_{\mathrm{C}-\mathrm{F}}=251.3 \mathrm{~Hz},{ }^{2} \mathrm{~J}_{\mathrm{C}-\mathrm{F}}=15.9 \mathrm{~Hz}$ ), 104.5 (dt, C-F, ${ }^{2} \mathrm{~J}_{\mathrm{C}-\mathrm{F}}=36.9 \mathrm{~Hz},{ }^{3} \mathrm{~J}_{\mathrm{C}-\mathrm{F}}=$ $4.1 \mathrm{~Hz}$ ), 99.9 (ddd, C-F, ${ }^{2} J_{\mathrm{C}-\mathrm{F}}=31.7 \mathrm{~Hz},{ }^{2} \mathrm{~J}_{\mathrm{C}-\mathrm{F}}=24.6 \mathrm{~Hz},{ }^{3} \mathrm{~J}_{\mathrm{C}-\mathrm{F}}=4.1 \mathrm{~Hz}$ ), 18.6, 18.4, 10.5 (t, C-F, $\left.{ }^{4} J_{C-F}=1.8 \mathrm{~Hz}\right)$. HRMS (El-TOF) calcd. for $\left[\mathrm{C}_{12} \mathrm{H}_{17} \mathrm{~F}_{3} \mathrm{Si}\right]\left([\mathrm{M}]^{+}\right): 246.1052$, found: 246.1051 .<smiles>Fc1cc(F)c([SiH](c2ccccc2)c2ccccc2)cc1F</smiles>

Diphenyl(2,4,5-trifluorophenyl)silane (2t). 1-Bromo-2,4,5-trifluorobenzene (1.2 mL, 10 mmol) was subjected to method B to give the corresponding hydrosilane as a colorless liquid (1.2 g, 38\%). $\mathrm{R}_{f}=0.9$ (petroleum ether). ${ }^{1} \mathrm{H}$ NMR $\left(400 \mathrm{MHz}, \mathrm{CDCl}_{3}\right): \delta 7.64-.7 .61$ (m, 4H), $7.52-7.42(\mathrm{~m}, 6 \mathrm{H}), 7.20$ (td, $J=9.6,4.8 \mathrm{~Hz}), 6.98-.6 .92(\mathrm{~m}) .{ }^{19} \mathrm{~F} \mathrm{NMR}(376 \mathrm{MHz}$, 
$\left.\mathrm{CDCl}_{3}\right): \delta-99.9--100.0(\mathrm{~m}),-129.9--130.0(\mathrm{~m}),-143.1--143.3(\mathrm{~m}) .{ }^{13} \mathrm{C}$ NMR $(101$ $\mathrm{MHz}, \mathrm{CDCl}_{3}$ ): $\delta 162.4\left(\mathrm{ddd}, \mathrm{C}-\mathrm{F},{ }^{1} \mathrm{~J}_{\mathrm{C}-\mathrm{F}}=242.8 \mathrm{~Hz},{ }^{3} \mathrm{~J}_{\mathrm{C}-\mathrm{F}}=9.5 \mathrm{~Hz},{ }^{4} \mathrm{~J}_{\mathrm{C}-\mathrm{F}}=2.0 \mathrm{~Hz}\right.$ ), 152.2 (dt, C-F, ${ }^{1} J_{\mathrm{C}-\mathrm{F}}=255.1 \mathrm{~Hz},{ }^{2} \mathrm{~J}_{\mathrm{C}-\mathrm{F}}=14.5 \mathrm{~Hz}$ ), 147.4 (ddd, C-F, ${ }^{1} \mathrm{~J}_{\mathrm{C}-\mathrm{F}}=247.6 \mathrm{~Hz},{ }^{2} \mathrm{~J}_{\mathrm{C}-\mathrm{F}}=$ $\left.11.9 \mathrm{~Hz},{ }^{3} \mathrm{~J}_{\mathrm{C}-\mathrm{F}}=3.8 \mathrm{~Hz}\right), 135.8,131.4,130.5,128.4,124.5$ (ddd, C-F, ${ }^{2} \mathrm{~J}_{\mathrm{C}-\mathrm{F}}=17.8 \mathrm{~Hz},{ }^{2} \mathrm{~J}_{\mathrm{C}}$ $\left.\mathrm{F}=12.4 \mathrm{~Hz},{ }^{4} \mathrm{~J}_{\mathrm{C}-\mathrm{F}}=1.2 \mathrm{~Hz}\right), 117.1\left(\mathrm{dt}, \mathrm{C}-\mathrm{F},{ }^{2} \mathrm{~J}_{\mathrm{C}-\mathrm{F}}=33.0 \mathrm{~Hz},{ }^{3} \mathrm{~J}_{\mathrm{C}-\mathrm{F}}=3.6 \mathrm{~Hz}\right), 105.7$ (dd, C$\mathrm{F},{ }^{2} \mathrm{~J}_{\mathrm{C}-\mathrm{F}}=31.8 \mathrm{~Hz},{ }^{2} \mathrm{~J}_{\mathrm{C}-\mathrm{F}}=20.2 \mathrm{~Hz}$ ). HRMS (El-TOF) calcd. for $\left[\mathrm{C}_{18} \mathrm{H}_{13} \mathrm{~F}_{3} \mathrm{Si}\right]\left([\mathrm{M}]^{+}\right)$: 314.0739, found: 314.0733 .<smiles>C[SiH](C)c1c(F)cccc1F</smiles>

(2,6-Difluorophenyl)dimethylsilane (2u). 2-Bromo-1,3-difluorobenzene (1.13 mL, 10 $\mathrm{mmol}$ ) was subjected to method $\mathrm{B}$ to give the corresponding hydrosilane as a colorless liquid $(1.0 \mathrm{~g}, 58 \%) . \mathrm{R}_{f}=0.9$ (petroleum ether). ${ }^{1} \mathrm{H}$ NMR $\left(400 \mathrm{MHz}, \mathrm{CDCl}_{3}\right) \delta 7.40-$ $7.27(\mathrm{~m}, 1 \mathrm{H}), 6.84(\mathrm{t}, J=7.5 \mathrm{~Hz}, 2 \mathrm{H}), 4.70-4.59(\mathrm{~m}, 1 \mathrm{H}), 0.45(\mathrm{~d}, J=3.9 \mathrm{~Hz}, 6 \mathrm{H}) .{ }^{19} \mathrm{~F}$ $\operatorname{NMR}\left(376 \mathrm{MHz}, \mathrm{CDCl}_{3}\right) \delta-97.98(\mathrm{~m}) .{ }^{13} \mathrm{C} \mathrm{NMR}\left(101 \mathrm{MHz}, \mathrm{CDCl}_{3}\right) \delta 168.4\left(\mathrm{~d}, \mathrm{C}-\mathrm{F},{ }^{2} \mathrm{~J}_{\mathrm{C}}\right.$ $\mathrm{F}=15.0 \mathrm{~Hz}), 166.0\left(\mathrm{~d}, \mathrm{C}-\mathrm{F},{ }^{2} \mathrm{~J}_{\mathrm{C}-\mathrm{F}}=15.1 \mathrm{~Hz}\right), 132.4\left(\mathrm{t}, \mathrm{C}-\mathrm{F},{ }^{3} \mathrm{~J}_{\mathrm{C}-\mathrm{F}}=10.4 \mathrm{~Hz}\right), 111.26-$ $110.74(\mathrm{~m}),-3.4\left(\mathrm{t}, \mathrm{C}-\mathrm{F},{ }^{4} \mathrm{~J}_{\mathrm{C}-\mathrm{F}}=2.6 \mathrm{~Hz}\right) . \mathrm{HRMS}$ (El-TOF) calcd. for $\left[\mathrm{C}_{8} \mathrm{H}_{10} \mathrm{~F}_{2} \mathrm{Si}\right]\left([\mathrm{M}]^{+}\right)$: 172.0520, found: 172.0526 .<smiles>COc1cc(F)c([SiH](C)C)c(F)c1</smiles>

(2,6-Difluoro-4-methoxyphenyl)dimethylsilane (2v). 2-Bromo-1,3-difluoro-5-methoxybenzene $(2.23 \mathrm{~g}, 10 \mathrm{mmol}$ ) was subjected to method $\mathrm{B}$ to give the corresponding hydrosilane as a colorless liquid (1.65 g, 82\%). $\mathrm{R}_{f}=0.9$ (petroleum ether). ${ }^{1} \mathrm{H}$ NMR $\left(400 \mathrm{MHz}, \mathrm{CDCl}_{3}\right) \delta 6.37(\mathrm{~d}, J=8.9 \mathrm{~Hz}, 2 \mathrm{H}), 4.56-4.49(\mathrm{~m}, 1 \mathrm{H}), 3.77(\mathrm{~s}, 3 \mathrm{H}), 0.37$ $(\mathrm{d}, J=3.8 \mathrm{~Hz}, 6 \mathrm{H}) .{ }^{19} \mathrm{~F}$ NMR $\left(376 \mathrm{MHz}, \mathrm{CDCl}_{3}\right) \delta-97.41(\mathrm{~m}) .{ }^{13} \mathrm{C} \mathrm{NMR}(101 \mathrm{MHz}$, $\left.\mathrm{CDCl}_{3}\right) \delta 169.1\left(\mathrm{~d}, \mathrm{C}-\mathrm{F},{ }^{2} J_{\mathrm{C}-\mathrm{F}}=19.2 \mathrm{~Hz}\right), 166.7\left(\mathrm{~d}, \mathrm{C}-\mathrm{F},{ }^{2} J_{\mathrm{C}-\mathrm{F}}=19.2 \mathrm{~Hz}\right), 163.3(\mathrm{t}, \mathrm{C}-$ $\left.\mathrm{F},{ }^{2} \mathrm{~J}_{\mathrm{C}-\mathrm{F}}=14.6 \mathrm{~Hz}\right), 102.6\left(\mathrm{t}, \mathrm{C}-\mathrm{F},{ }^{2} \mathrm{~J}_{\mathrm{C}-\mathrm{F}}=35.0 \mathrm{~Hz}\right), 97.8\left(\mathrm{dd}, \mathrm{C}-\mathrm{F},{ }^{2} \mathrm{~J}_{\mathrm{C}-\mathrm{F}}=31.5 \mathrm{~Hz},{ }^{4} \mathrm{~J}_{\mathrm{C}}\right.$ $\mathrm{F}=2.7 \mathrm{~Hz}), 55.8,-3.2\left(\mathrm{t}, \mathrm{C}-\mathrm{F},{ }^{4} \mathrm{~J}_{\mathrm{C}-\mathrm{F}}=2.6 \mathrm{~Hz}\right) . \mathrm{HRMS}(\mathrm{El}-\mathrm{TOF})$ calcd. for $\left[\mathrm{C}_{9} \mathrm{H}_{12} \mathrm{~F}_{2} \mathrm{OSi}\right]$ $\left([\mathrm{M}]^{+}\right): 202.0625$, found: 202.0624 . 
<smiles>Cc1ccccc1[Si](C)(C)c1c(F)c(F)c(F)c(F)c1F</smiles>

Methyl(perfluorophenyl)(o-tolyl)silane (5a). 2-Methylbromobenzene (0.60 mL, $5.0 \mathrm{mmol})$ and bromopentafluorobenzene $(0.62 \mathrm{~mL}, 5.0 \mathrm{mmol})$ was subjected to method $A$ to give the corresponding hydrosilane as a colorless liquid (0.69 g, 46\%). $R_{f}=0.9$ (petroleum ether). ${ }^{1} \mathrm{H} \mathrm{NMR}\left(400 \mathrm{MHz}, \mathrm{CDCl}_{3}\right): \delta 7.63(\mathrm{~d}, J=8.0 \mathrm{~Hz}, 1 \mathrm{H}), 7.42(\mathrm{t}, J=8.0 \mathrm{~Hz}, 1 \mathrm{H}), 7.32-7.26$ $(\mathrm{m}, 2 \mathrm{H}), 5.37-5.32(\mathrm{~m}, 1 \mathrm{H}), 2.52(\mathrm{~s}, 3 \mathrm{H}), 0.86(\mathrm{~d}, J=4.0 \mathrm{~Hz}, 3 \mathrm{H}) .{ }^{19} \mathrm{~F}$ NMR $(376 \mathrm{MHz}$, $\left.\mathrm{CDCl}_{3}\right): \delta-126.4--126.5(\mathrm{~m}),-150.7--150.8(\mathrm{~m}),-160.8--161.0(\mathrm{~m}) .{ }^{13} \mathrm{C}$ NMR $(101$ $\left.\mathrm{MHz}, \mathrm{CDCl}_{3}\right): \delta 149.0\left(\mathrm{dm}, \mathrm{C}-\mathrm{F},{ }^{1} \mathrm{~J}_{\mathrm{C}-\mathrm{F}}=244.0 \mathrm{~Hz}\right), 144.0,142.5\left(\mathrm{dm}, \mathrm{C}-\mathrm{F},{ }^{1} \mathrm{~J}_{\mathrm{C}-\mathrm{F}}=256.3\right.$ $\mathrm{Hz}), 137.3\left(\mathrm{dm}, \mathrm{C}-\mathrm{F},{ }^{1} \mathrm{~J}_{\mathrm{C}-\mathrm{F}}=255.1 \mathrm{~Hz}\right), 135.2,130.9,130.7,129.8,125.5,107.9-107.8$ (m), 22.2, -5.22 (t, C-F, $\left.{ }^{4} J_{C-F}=3.0 \mathrm{~Hz}\right)$. IR (thin film): 2970, 2174, 1514, 1460, 1132, 969, 821, 738. HRMS (El-TOF) calcd. for $\left[\mathrm{C}_{14} \mathrm{H}_{11} \mathrm{~F}_{5} \mathrm{Si}\right]\left([\mathrm{M}]^{+}\right)$: 302.0550, found: 302.0560.<smiles>C[Si](c1ccccc1)(c1ccc(-c2ccccc2)cc1)c1c(F)c(F)c(F)c(F)c1F</smiles>

[1,1'-Biphenyl]-4-yl(methyl)(perfluorophenyl)silane (5b). 4-Bromobiphenyl (2.3 g, 10 $\mathrm{mmol}$ ) and bromopentafluorobenzene $(1.2 \mathrm{~mL}, 10 \mathrm{mmol})$ was subjected to method $\mathrm{A}$ to give the corresponding hydrosilane as a colorless liquid $(1.5 \mathrm{~g}, 40 \%) . R_{f}=0.9$ (petroleum ether). ${ }^{1} \mathrm{H}$ NMR (400 MHz, $\left.\mathrm{CDCl}_{3}\right): \delta 7.70(\mathrm{~d}, J=8.0 \mathrm{~Hz}, 2 \mathrm{H}), 7.65-7.60(\mathrm{~m}, 4 \mathrm{H}), 7.47$ (t, $J=8.0 \mathrm{~Hz}, 2 \mathrm{H}), 7.39(\mathrm{t}, J=8.0 \mathrm{~Hz}, 1 \mathrm{H}), 5.22-5.17(\mathrm{~m}, 1 \mathrm{H}), 0.81(\mathrm{~d}, J=4.0 \mathrm{~Hz}, 3 \mathrm{H}) .{ }^{19} \mathrm{~F}$ $\operatorname{NMR}\left(376 \mathrm{MHz}, \mathrm{CDCl}_{3}\right)$ : $\delta-126.2--126.3(\mathrm{~m}),-150.3--150.4(\mathrm{~m}),-160.6--160.7(\mathrm{~m})$. ${ }^{13} \mathrm{C}$ NMR $\left(101 \mathrm{MHz}, \mathrm{CDCl}_{3}\right): \delta 149.1\left(\mathrm{dm}, \mathrm{C}-\mathrm{F},{ }^{1} \mathrm{~J}_{\mathrm{C}-\mathrm{F}}=248.9 \mathrm{~Hz}\right), 143.3,142.5(\mathrm{dm}, \mathrm{C}-\mathrm{F}$, $\left.{ }^{1} J_{C-F}=260.2 \mathrm{~Hz}\right), 140.7,137.4\left(\mathrm{dm}, \mathrm{C}-\mathrm{F},{ }^{1} \mathrm{~J}_{\mathrm{C}-\mathrm{F}}=254.7 \mathrm{~Hz}\right), 135.1,130.8,129.0,127.8$, 127.3, 127.1, $108.1-107.5(\mathrm{~m}),-5.3\left(\mathrm{t}, \mathrm{C}-\mathrm{F},{ }^{4} \mathrm{~J}_{\mathrm{C}-\mathrm{F}}=3.2 \mathrm{~Hz}\right) . \mathrm{IR}$ (thin film): 2171,1514 , 1461, 1082, 968, 819, 740. HRMS (El-TOF) calcd. for $\left[\mathrm{C}_{19} \mathrm{H}_{13} \mathrm{~F}_{5} \mathrm{Si}\right]\left([\mathrm{M}]^{+}\right): 364.0707$, found: 364.0714 . 
<smiles>C[Si](C)(c1ccc2ccccc2c1)c1c(F)c(F)c(F)c(F)c1F</smiles>

Methyl(naphthalen-2-yl)(perfluorophenyl)silane (5c). 2-Bromonaphthalene (1.0 g, 5.0 $\mathrm{mmol})$ and bromopentafluorobenzene $(0.62 \mathrm{~mL}, 5.0 \mathrm{mmol})$ was subjected to method $A$ to give the corresponding hydrosilane as a colorless liquid $(0.50 \mathrm{~g}, 30 \%) . \mathrm{R}_{f}=0.9$ (petroleum ether). ${ }^{1} \mathrm{H}$ NMR (400 MHz, $\mathrm{CDCl}_{3}$ ): $\delta 8.22$ (s, 1H), 7.91 (t, $J=8.0 \mathrm{~Hz}, 3 \mathrm{H}$ ), 7.72 (d, $J=8.0$ $\mathrm{Hz}, 1 \mathrm{H}), 7.60-7.56(\mathrm{~m}, 2 \mathrm{H}), 5.37-5.33(\mathrm{~m}, 1 \mathrm{H}), 0.92(\mathrm{~d}, J=4.0 \mathrm{~Hz}, 3 \mathrm{H}) .{ }^{19} \mathrm{~F}$ NMR $(376$ $\left.\mathrm{MHz}, \mathrm{CDCl}_{3}\right): \delta-126.1--126.2(\mathrm{~m}),-150.2--150.4(\mathrm{~m}),-160.5--160.6(\mathrm{~m}) .{ }^{13} \mathrm{C}$ NMR $\left(101 \mathrm{MHz}, \mathrm{CDCl}_{3}\right): \delta 149.1\left(\mathrm{dm}, \mathrm{C}-\mathrm{F},{ }^{1} \mathrm{~J}_{\mathrm{C}-\mathrm{F}}=239.1 \mathrm{~Hz}\right), 142.6\left(\mathrm{dm}, \mathrm{C}-\mathrm{F},{ }^{1} \mathrm{~J}_{\mathrm{C}-\mathrm{F}}=256.4\right.$ $\mathrm{Hz}), 137.4$ (dm, C-F, $\left.{ }^{1} \mathrm{~J}_{\mathrm{C}-\mathrm{F}}=255.0 \mathrm{~Hz}\right), 135.9,134.3,133.0,130.0,128.3,127.9,127.8$, 127.2, 126.5, 125.9, $108.1-107.8(\mathrm{~m}),-5.2\left(\mathrm{t}, \mathrm{C}-\mathrm{F},{ }^{4} J_{\mathrm{C}-\mathrm{F}}=3.2 \mathrm{~Hz}\right)$. IR (thin film): 2171 , 1514, 1461, 1083, 969, 815, 743. HRMS (El-TOF) calcd. for $\left[\mathrm{C}_{17} \mathrm{H}_{11} \mathrm{~F}_{5} \mathrm{Si}\right]\left([\mathrm{M}]^{+}\right)$: 338.0550 , found: 338.0547 .<smiles>COc1ccc2ccccc2c1[Si](C)(C)C(F)(F)F</smiles>

(2-Methoxynaphthalen-1-yl)(methyl)(perfluorophenyl)silane (5d). 1-Bromo-2-methoxynaphthalene $(1.2 \mathrm{~g}, 5.0 \mathrm{mmol})$ and bromopentafluorobenzene $(0.62 \mathrm{~mL}, 5.0 \mathrm{mmol})$ was subjected to method A to give the corresponding hydrosilane as a white solid ( $0.81 \mathrm{~g}, 44 \%)$. $\mathrm{R}_{f}=0.9$ (petroleum ether). M. P.: $73-75^{\circ} \mathrm{C} .{ }^{1} \mathrm{H}$ NMR $\left(400 \mathrm{MHz}, \mathrm{CDCl}_{3}\right): \delta 8.43(\mathrm{~d}, J=8.0$ $\mathrm{Hz}, 1 \mathrm{H}), 7.98(\mathrm{~d}, J=8.0 \mathrm{~Hz}, 1 \mathrm{H}), 7.86(\mathrm{~d}, J=8.0 \mathrm{~Hz}, 1 \mathrm{H}), 7.58(\mathrm{~s}, 1 \mathrm{H}), 7.42(\mathrm{~s}, 1 \mathrm{H}), 7.23$ (d, $J=8.0 \mathrm{~Hz}, 1 \mathrm{H}), 5.73(\mathrm{~s}, 1 \mathrm{H}), 3.90(\mathrm{~s}, 3 \mathrm{H}), 0.90(\mathrm{~s}, 3 \mathrm{H}) .{ }^{19} \mathrm{~F} \mathrm{NMR}\left(376 \mathrm{MHz}, \mathrm{CDCl}_{3}\right): \delta$ $-127.3(\mathrm{~d}, J=4.4 \mathrm{~Hz}),-152.2(\mathrm{t}, J=19.2 \mathrm{~Hz}),-161.9--162.0(\mathrm{~m}) .{ }^{13} \mathrm{C}$ NMR $(101 \mathrm{MHz}$, $\left.\mathrm{CDCl}_{3}\right): \delta$ 163.6, $149.2\left(\mathrm{dm}, \mathrm{C}-\mathrm{F},{ }^{1} \mathrm{~J}_{\mathrm{C}-\mathrm{F}}=238.7 \mathrm{~Hz}\right), 139.6\left(\mathrm{dm}, \mathrm{C}-\mathrm{F},{ }^{1} \mathrm{~J}_{\mathrm{C}-\mathrm{F}}=261.1 \mathrm{~Hz}\right)$, 137.9, 137.2 (dm, C-F, $\left.{ }^{1} \mathrm{~J}-\mathrm{F}=245.9 \mathrm{~Hz}\right), 133.6,129.5,128.9,127.2,126.5,123.7,114.2$, 112.3, 109.0 - 108.9 (m), 56.0, -3.9. IR (thin film): 2196, 1458, 1377, 1266, 1144, 963, 809. HRMS (El-TOF) calcd. for $\left[\mathrm{C}_{18} \mathrm{H}_{13} \mathrm{~F}_{5} \mathrm{OSi}\right]\left([\mathrm{M}]^{+}\right)$: 368.0656 , found: 368.0667 . 
<smiles>C[Si](C)(c1ccc(-n2cccc2)cc1)c1c(F)c(F)c(F)c(F)c1F</smiles>

1-(4-(Methyl(perfluorophenyl)silyl)phenyl)-1 H-pyrrole (5e). 1-(4-Bromophenyl)-1 H-pyrrole $(1.0 \mathrm{~g}, 4.5 \mathrm{mmol})$ and bromopentafluorobenzene $(0.62 \mathrm{~mL}, 5.0 \mathrm{mmol})$ was subjected to method $A$ to give the corresponding hydrosilane as a white solid $(0.25 \mathrm{~g}, 16 \%) . R_{f}=0.9$ (petroleum ether). ${ }^{1} \mathrm{H}$ NMR $\left(400 \mathrm{MHz}, \mathrm{CDCl}_{3}\right): \delta 7.69(\mathrm{~d}, J=8.0 \mathrm{~Hz}, 2 \mathrm{H}), 7.45(\mathrm{~d}, J=8.0$ $\mathrm{Hz}, 2 \mathrm{H}), 7.15(\mathrm{~s}, 2 \mathrm{H}), 6.40(\mathrm{~s}, 2 \mathrm{H}), 5.22-5.17(\mathrm{~m}, 1 \mathrm{H}), 0.82(\mathrm{~d}, J=4.0 \mathrm{~Hz}, 3 \mathrm{H}) .{ }^{19} \mathrm{~F} \mathrm{NMR}$ $\left(376 \mathrm{MHz}, \mathrm{CDCl}_{3}\right): \delta-126.3--126.4(\mathrm{~m}),-150.1--150.2(\mathrm{~m}),-160.4--160.6(\mathrm{~m}) .{ }^{13} \mathrm{C}$ $\operatorname{NMR}\left(101 \mathrm{MHz}, \mathrm{CDCl}_{3}\right): \delta 148.9\left(\mathrm{dm}, \mathrm{C}-\mathrm{F},{ }^{1} \mathrm{~J}_{\mathrm{C}-\mathrm{F}}=248.3 \mathrm{~Hz}\right), 142.6\left(\mathrm{dm}, \mathrm{C}-\mathrm{F},{ }^{1} \mathrm{~J}_{\mathrm{C}-\mathrm{F}}=\right.$ $256.3 \mathrm{~Hz}), 142.3,137.3\left(\mathrm{dm}, \mathrm{C}-\mathrm{F},{ }^{1} \mathrm{~J}_{\mathrm{C}-\mathrm{F}}=255.0 \mathrm{~Hz}\right), 136.0,128.8,119.9,119.0,110.9$, $107.8-107.2(\mathrm{~m}),-5.3\left(\mathrm{t}, \mathrm{C}-\mathrm{F},{ }^{4} \mathrm{~J}_{\mathrm{C}-\mathrm{F}}=3.2 \mathrm{~Hz}\right) . \mathrm{IR}$ (thin film): $2177,1457,1328,1085,964$, 813, 718. HRMS (EI-TOF) calcd. for [ $\left.\mathrm{C}_{17} \mathrm{H}_{12} \mathrm{~F}_{5} \mathrm{NSi}\right]\left([\mathrm{M}]^{+}\right)$: 353.0659, found: 353.0666 .<smiles>C[Si](c1ccsc1)c1c(F)c(F)c(F)c(F)c1F</smiles>

Methyl(perfluorophenyl)(thiophen-3-yl)silane (5f). 3-Bromothiophene (0.47 mL, 5.0 $\mathrm{mmol})$ and bromopentafluorobenzene $(0.62 \mathrm{~mL}, 5.0 \mathrm{mmol})$ was subjected to method $A$ to give the corresponding hydrosilane as a colorless liquid $(0.20 \mathrm{~g}, 14 \%) . R_{f}=0.9$ (petroleum ether). ${ }^{1} \mathrm{H}$ NMR (400 MHz, $\mathrm{CDCl}_{3}$ ): $\delta 7.72(\mathrm{~s}, 1 \mathrm{H}), 7.48-7.47(\mathrm{~m}, 1 \mathrm{H}), 7.34(\mathrm{~d}, J=4.0 \mathrm{~Hz}$, 1H), $5.28-5.25(\mathrm{~m}, 1 \mathrm{H}), 0.84(\mathrm{~d}, J=4.0 \mathrm{~Hz}, 3 \mathrm{H}) .{ }^{19} \mathrm{~F} \mathrm{NMR}\left(376 \mathrm{MHz}, \mathrm{CDCl}_{3}\right): \delta-126.6-$ $-126.7(\mathrm{~m}),-150.6--150.7(\mathrm{~m}),-160.8--160.9(\mathrm{~m}) .{ }^{13} \mathrm{C}$ NMR $\left(101 \mathrm{MHz}, \mathrm{CDCl}_{3}\right): \delta$ $149.0\left(\mathrm{dm}, \mathrm{C}-\mathrm{F},{ }^{1} \mathrm{~J}_{\mathrm{C}-\mathrm{F}}=243.6 \mathrm{~Hz}\right), 142.7\left(\mathrm{dm}, \mathrm{C}-\mathrm{F},{ }^{1} \mathrm{~J}_{\mathrm{C}-\mathrm{F}}=256.3 \mathrm{~Hz}\right), 137.4(\mathrm{dm}, \mathrm{C}-\mathrm{F}$, $\left.{ }^{1} J_{C-F}=254.6 \mathrm{~Hz}\right), 135.0,132.2,131.8,126.7,108.2-107.5(\mathrm{~m}),-4.8\left(\mathrm{t}, \mathrm{C}-\mathrm{F},{ }^{4} \mathrm{~J}_{\mathrm{C}-\mathrm{F}}=2.7\right.$ Hz). IR (thin film): 2180, 1516, 1463, 10856, 971, 822, 716. HRMS (El-TOF) calcd. for $\left[\mathrm{C}_{11} \mathrm{H}_{7} \mathrm{~F}_{5} \mathrm{SSi}\right]\left([\mathrm{M}]^{+}\right):$293.9958, found: 293.9959.<smiles>C[Si](c1ccc2sccc2c1)(c1cccs1)c1c(F)c(F)c(F)c(F)c1F</smiles> 
Benzo[b]thiophen-5-yl(methyl)(perfluorophenyl)silane $\quad(5 \mathrm{~g})$. 5-Bromobenzo[b]thiophene $(1.1 \mathrm{~g}, 5.0 \mathrm{mmol})$ and bromopentafluorobenzene $(0.62 \mathrm{~mL}, 5.0 \mathrm{mmol})$ was subjected to method $A$ to give the corresponding hydrosilane as a colorless liquid $(0.31 \mathrm{~g}, 18 \%)$. $R_{f}=$ 0.9 (petroleum ether). ${ }^{1} \mathrm{H}$ NMR $\left(400 \mathrm{MHz}, \mathrm{CDCl}_{3}\right): \delta 8.13(\mathrm{~d}, J=8.0 \mathrm{~Hz}, 1 \mathrm{H}), 7.95$ (d, $J=$ $8.0 \mathrm{~Hz}, 1 \mathrm{H}), 7.57(\mathrm{t}, J=8.0 \mathrm{~Hz}, 1 \mathrm{H}), 7.48(\mathrm{~d}, J=8.0 \mathrm{~Hz}, 1 \mathrm{H}), 7.38(\mathrm{~d}, J=4.0 \mathrm{~Hz}, 1 \mathrm{H}), 5.30$ - $5.25(\mathrm{~m}, 1 \mathrm{H}), 0.88(\mathrm{~d}, J=4.0 \mathrm{~Hz}, 3 \mathrm{H}) .{ }^{19} \mathrm{~F} \mathrm{NMR}\left(376 \mathrm{MHz}, \mathrm{CDCl}_{3}\right): \delta-126.2--126.3$ (m), $-150.3--150.4(\mathrm{~m}),-160.5--160.6(\mathrm{~m}) .{ }^{13} \mathrm{C}$ NMR (101 MHz, CDCl $)$ : $\delta 148.9(\mathrm{dm}$, $\left.\mathrm{C}-\mathrm{F},{ }^{1} \mathrm{~J}_{\mathrm{C}-\mathrm{F}}=243.8 \mathrm{~Hz}\right), 142.5\left(\mathrm{dm}, \mathrm{C}-\mathrm{F},{ }^{1} \mathrm{~J}_{\mathrm{C}-\mathrm{F}}=256.2 \mathrm{~Hz}\right), 141.9,139.4,137.3(\mathrm{dm}, \mathrm{C}-\mathrm{F}$, $\left.{ }^{1} J_{C-F}=255.0 \mathrm{~Hz}\right), 130.3,129.2,127.2,126.8,123.8,122.5,108.2-107.5(\mathrm{~m}),-5.2(\mathrm{t}, \mathrm{C}-$ $\mathrm{F},{ }^{4} J_{\mathrm{C}-\mathrm{F}}=3.3 \mathrm{~Hz}$ ). IR (thin film): $2171,1514,1460,1081,968,848,805 . \mathrm{HRMS}$ (EI-TOF) calcd. for $\left[\mathrm{C}_{15} \mathrm{H}_{9} \mathrm{~F}_{5} \mathrm{SSi}\right]\left([\mathrm{M}]^{+}\right): 344.0114$, found: 344.0115 . 


\section{3. $\mathrm{Ru}(\mathrm{II})$-Catalyzed ortho-Hydrodefluorination of Polyfluorophenyl Hy- drosilanes}

\section{General procedure}

In an argon-filled glovebox, a $5 \mathrm{~mL}$ dried Schlenk tube was charged with the desired amount of Ru complex, hydrosilane, and THF (or neat). The tube was sealed tightly with a teflon plug and the mixture was stirred at $120^{\circ} \mathrm{C}$ for $24 \mathrm{~h}$. After the resulting reaction mixture was cooled to room temperature, pentafluorobenzene (1.0 equiv) was added as an internal standard and the NMR yield was determined by ${ }^{19} \mathrm{~F}$ NMR spectra. The crude mixture was purified by flash column chromatography (silica gel, petroleum ether : ethyl acetate $=15: 1$ ) or Kugelrohr distillation to obtain the hydrodefluorination product.

\section{Spectral data for the products of intramolecular ortho-hydrodefluorination}<smiles>C[Si](c1ccccc1)(c1ccccc1)c1cc(F)c(F)c(F)c1F</smiles>

Fluoro(methyl)(phenyl)(2,3,4,5-tetrafluorophenyl)silane (3a). The general procedure was followed with Ru complex 1b (2.2 mg, $4.1 \mu \mathrm{mol}, 1.0 \mathrm{~mol} \%)$ and hydrosilane $2 \mathrm{a}$ (115.2 $\mathrm{mg}, 0.40 \mathrm{mmol}$ ) at $120^{\circ} \mathrm{C}$ for $24 \mathrm{~h}$. The hydrodefluorination product was afforded as a colorless liquid (106.2 mg, 92\% yield). ${ }^{1} \mathrm{H}$ NMR (400 MHz, $\left.\mathrm{CDCl}_{3}\right): \delta 7.62$ (d, J = 8.0 Hz, 2H), $7.52(\mathrm{t}, J=8.0 \mathrm{~Hz}, 1 \mathrm{H}), 7.45(\mathrm{t}, J=8.0 \mathrm{~Hz}, 2 \mathrm{H}), 7.11-7.05(\mathrm{~m}, 1 \mathrm{H}), 0.85(\mathrm{~d}, J=8.0$ $\mathrm{Hz}, 3 \mathrm{H}) .{ }^{19} \mathrm{~F}$ NMR $\left(376 \mathrm{MHz}, \mathrm{CDCl}_{3}\right): \delta-127.0(\mathrm{t}, J=18.8 \mathrm{~Hz}),-137.9--138.1(\mathrm{~m}),-$ $151.2--151.4(\mathrm{~m}),-154.8(\mathrm{t}, J=19.9 \mathrm{~Hz}),-166.6 .{ }^{13} \mathrm{C} \mathrm{NMR}\left(101 \mathrm{MHz}, \mathrm{CDCl}_{3}\right): \delta 150.7$ (dd, C-F, $\left.\left.{ }^{1} J_{C-F}=243.0 \mathrm{~Hz}\right),{ }^{2} J_{C-F}=10.3 \mathrm{~Hz}\right), 147.6\left(\mathrm{ddd}, \mathrm{C}-\mathrm{F},{ }^{1} \mathrm{~J}_{\mathrm{C}-\mathrm{F}}=251.7 \mathrm{~Hz},{ }^{2} \mathrm{~J}_{\mathrm{C}-\mathrm{F}}=\right.$ $\left.9.9 \mathrm{~Hz},{ }^{3} \mathrm{~J}_{\mathrm{C}-\mathrm{F}}=3.2 \mathrm{~Hz}\right), 142.3\left(\mathrm{dm}, \mathrm{C}-\mathrm{F},{ }^{1} \mathrm{~J}_{\mathrm{C}-\mathrm{F}}=258.3 \mathrm{~Hz}\right), 140.4\left(\mathrm{dm}, \mathrm{C}-\mathrm{F},{ }^{1} \mathrm{~J}_{\mathrm{C}-\mathrm{F}}=260.1\right.$ $\mathrm{Hz}), 133.5\left(\mathrm{~d}, \mathrm{C}-\mathrm{F},{ }^{4} \mathrm{~J}_{\mathrm{C}-\mathrm{F}}=1.5 \mathrm{~Hz}\right), 132.1\left(\mathrm{~d}, \mathrm{C}-\mathrm{F},{ }^{2} \mathrm{~J}_{\mathrm{C}-\mathrm{F}}=16.2 \mathrm{~Hz}\right), 131.4,128.4,118.0-$ $117.4(\mathrm{~m}), 115.6-115.2(\mathrm{~m}),-2.1\left(\mathrm{~d}, \mathrm{C}-\mathrm{F},{ }^{2} \mathrm{~J}_{\mathrm{C}-\mathrm{F}}=14.0 \mathrm{~Hz}\right) . \mathrm{IR}$ (thin film): 1452, 1092 , 1009, 770, 727, 697. HRMS (El-TOF) calcd. for $\left[\mathrm{C}_{13} \mathrm{H}_{9} \mathrm{~F}_{5} \mathrm{Si}\right]\left([\mathrm{M}]^{+}\right)$: 288.0394 , found: 288.0405 . 
<smiles>COc1ccccc1[Si](C)(C)c1cc(F)c(F)c(F)c1F</smiles>

Fluoro(2-methoxyphenyl)(methyl)(2,3,4,5-tetrafluorophenyl)silane (3b). The general procedure was followed with Ru complex 1b (42.6 mg, $8.0 \mu \mathrm{mol}, 1.0 \mathrm{~mol} \%)$ and hydrosilane $2 \mathrm{~b}(2.55 \mathrm{~g}, 8.0 \mathrm{mmol})$ at $120^{\circ} \mathrm{C}$ for $24 \mathrm{~h} .{ }^{1} \mathrm{H} \mathrm{NMR}\left(400 \mathrm{MHz}, \mathrm{CDCl}_{3}\right): \delta 7.58$ (d, $J=8.0 \mathrm{~Hz}, 1 \mathrm{H}), 7.53(\mathrm{t}, J=8.0 \mathrm{~Hz}, 1 \mathrm{H}), 7.11-6.93(\mathrm{~m}, 2 \mathrm{H}), 6.94(\mathrm{~d}, J=8.0 \mathrm{~Hz}, 1 \mathrm{H}), 3.81$ (s, 3H), 0.88 (d, $J=4.0 \mathrm{~Hz}, 3 \mathrm{H}) .{ }^{19} \mathrm{~F} \mathrm{NMR}\left(376 \mathrm{MHz}, \mathrm{CDCl}_{3}\right): \delta-127.6$ (t, $\left.J=18.4 \mathrm{~Hz}\right),-$ $139.0--139.1(\mathrm{~m}),-152.3--152.4(\mathrm{~m}),-155.6(\mathrm{t}, J=19.9 \mathrm{~Hz}),-166.4--166.5(\mathrm{~m}) .{ }^{13} \mathrm{C}$ $\operatorname{NMR}\left(101 \mathrm{MHz}, \mathrm{CDCl}_{3}\right): \delta 164.1\left(\mathrm{~d}, \mathrm{C}-\mathrm{F},{ }^{3} \mathrm{~J}_{\mathrm{C}-\mathrm{F}}=2.6 \mathrm{~Hz}\right), 150.8\left(\mathrm{dm}, \mathrm{C}-\mathrm{F},{ }^{1} \mathrm{~J}_{\mathrm{C}-\mathrm{F}}=243.8\right.$ $\mathrm{Hz}$ ), 147.3 (ddd, C-F, $\left.{ }^{1} \mathrm{~J}_{\mathrm{C}-\mathrm{F}}=250.5 \mathrm{~Hz},{ }^{2} \mathrm{~J}_{\mathrm{C}-\mathrm{F}}=10.0 \mathrm{~Hz},{ }^{3} \mathrm{~J}_{\mathrm{C}-\mathrm{F}}=3.2 \mathrm{~Hz}\right), 141.9(\mathrm{dm}, \mathrm{C}-\mathrm{F}$, $\left.{ }^{1} \mathrm{~J}_{\mathrm{C}-\mathrm{F}}=256.9 \mathrm{~Hz}\right), 140.2\left(\mathrm{dm}, \mathrm{C}-\mathrm{F},{ }^{1} \mathrm{~J}_{\mathrm{C}-\mathrm{F}}=258.0 \mathrm{~Hz}\right), 135.2,133.3,121.2-121.1(\mathrm{~m})$, $120.3\left(\mathrm{~d}, \mathrm{C}-\mathrm{F},{ }^{2} \mathrm{~J}_{\mathrm{C}-\mathrm{F}}=16.0 \mathrm{~Hz}\right), 118.6-118.1(\mathrm{~m}), 115.8-115.3(\mathrm{~m}), 109.9,55.1(\mathrm{~d}, \mathrm{~J}=$ $9.0 \mathrm{~Hz}$ ), $-1.4\left(\mathrm{dd}, \mathrm{C}-\mathrm{F},{ }^{2} J_{\mathrm{C}-\mathrm{F}}=14.5 \mathrm{~Hz},{ }^{4} J_{\mathrm{C}-\mathrm{F}}=2.1 \mathrm{~Hz}\right.$ ). IR (thin film): 1451, 1241, 1008 , 847, 786, 738.0. HRMS (El-TOF) calcd. for [ $\left.\mathrm{C}_{14} \mathrm{H}_{11} \mathrm{~F}_{5} \mathrm{OSi}\right]\left([\mathrm{M}]^{+}\right): 318.0499$, found: 318.0506 .<smiles>COc1cc(F)c([Si](C)(C)c2cc(F)c(F)c(F)c2F)c(F)c1</smiles>

(2,6-Difluoro-4-methoxyphenyl)fluoro(methyl)(2,3,4,5-tetrafluorophenyl)silane (3c). The general procedure was followed with Ru complex $1 \mathrm{~b}$ (1.1 mg, $2.1 \mu \mathrm{mol}, 1.0 \mathrm{~mol} \%)$ and hydrosilane $2 \mathrm{j}(70.8 \mathrm{mg}, 0.20 \mathrm{mmol})$ at $120^{\circ} \mathrm{C}$ for $24 \mathrm{~h}$. The hydrodefluorination product was afforded as a colorless liquid (65.7 mg, 93\% yield). ${ }^{1} \mathrm{H} \mathrm{NMR}\left(400 \mathrm{MHz}, \mathrm{CDCl}_{3}\right): \delta$ $7.15-7.10(\mathrm{~m}, 1 \mathrm{H}), 6.43(\mathrm{~d}, J=8.0 \mathrm{~Hz}, 2 \mathrm{H}), 3.82(\mathrm{~s}, 3 \mathrm{H}), 0.88(\mathrm{~d}, J=4.0 \mathrm{~Hz}, 3 \mathrm{H}) .{ }^{19} \mathrm{~F}$ NMR (376 MHz, $\left.\mathrm{CDCl}_{3}\right): \delta-96.8(\mathrm{t}, J=9.8 \mathrm{~Hz}),-128.1$ (t, $\left.J=18.4 \mathrm{~Hz}\right),-138.3--138.3$ (m), $-151.3--151.5(\mathrm{~m}),-155.2(\mathrm{t}, J=20.7 \mathrm{~Hz}),-162.0--162.1(\mathrm{~m}) .{ }^{13} \mathrm{C}$ NMR $(101 \mathrm{MHz}$, $\left.\mathrm{CDCl}_{3}\right): \delta 167.9\left(\mathrm{dd}, \mathrm{C}-\mathrm{F},{ }^{1} \mathrm{~J}_{\mathrm{C}-\mathrm{F}}=246.6 \mathrm{~Hz},{ }^{2} \mathrm{~J}_{\mathrm{C}-\mathrm{F}}=17.8 \mathrm{~Hz}\right), 165.3\left(\mathrm{t}, \mathrm{C}-\mathrm{F},{ }^{2} \mathrm{~J}_{\mathrm{C}-\mathrm{F}}=14.8\right.$ $\mathrm{Hz}), 150.8\left(\mathrm{dm}, \mathrm{C}-\mathrm{F},{ }^{1} \mathrm{~J}_{\mathrm{C}-\mathrm{F}}=243.8 \mathrm{~Hz}\right), 147.7$ (ddd, C-F, ${ }^{1} \mathrm{~J}_{\mathrm{C}-\mathrm{F}}=251.1 \mathrm{~Hz},{ }^{2} J_{\mathrm{C}-\mathrm{F}}=10.3$ $\left.\mathrm{Hz},{ }^{3} \mathrm{~J}_{\mathrm{C}-\mathrm{F}}=3.5 \mathrm{~Hz}\right), 142.5\left(\mathrm{dm}, \mathrm{C}-\mathrm{F},{ }^{1} \mathrm{~J}_{\mathrm{C}-\mathrm{F}}=258.4 \mathrm{~Hz}\right), 140.4\left(\mathrm{dm}, \mathrm{C}-\mathrm{F},{ }^{1} J_{\mathrm{C}-\mathrm{F}}=258.6 \mathrm{~Hz}\right)$, 
$118.1-117.6(\mathrm{~m}), 115.4-115.1(\mathrm{~m}), 99.1-98.2(\mathrm{~m}), 98.3\left(\mathrm{dd}, \mathrm{C}-\mathrm{F},{ }^{2} \mathrm{~J}_{\mathrm{C}-\mathrm{F}}=30.1 \mathrm{~Hz},{ }^{4} \mathrm{~J}_{\mathrm{C}}\right.$ $\mathrm{F}=2.7 \mathrm{~Hz}$ ), 56.0, $0.2\left(\mathrm{dd}, \mathrm{C}-\mathrm{F},{ }^{2} \mathrm{~J}_{\mathrm{C}-\mathrm{F}}=13.7 \mathrm{~Hz},{ }^{4} \mathrm{~J}_{\mathrm{C}-\mathrm{F}}=2.3 \mathrm{~Hz}\right) . \mathrm{IR}$ (thin film): 1620, 1454 , 1326, 1111, 1092, 1009, 878. HRMS (El-TOF) calcd. for $\left[\mathrm{C}_{14} \mathrm{H}_{9} \mathrm{~F}_{7} \mathrm{OSi}\right]\left([\mathrm{M}]^{+}\right): 354.0311$, found: 354.0307 .<smiles>C[Si](c1cccs1)(c1cccs1)c1cc(F)c(F)c(F)c1F</smiles>

Fluoro(methyl)(2,3,4,5-tetrafluorophenyl)(thiophen-2-yl)silane (3d). The general procedure was followed with Ru complex $1 \mathrm{~b}(2.1 \mathrm{mg}, 3.9 \mu \mathrm{mol}, 1.0 \mathrm{~mol} \%)$ and hydrosilane 20 $(117.6 \mathrm{mg}, 0.40 \mathrm{mmol})$ at $120^{\circ} \mathrm{C}$ for $24 \mathrm{~h}$. The hydrodefluorination product was afforded as a colorless liquid (106.3 mg, 90\% yield). ${ }^{1} \mathrm{H}$ NMR (400 MHz, $\left.\mathrm{CDCl}_{3}\right): \delta 7.80$ (d, $J=4.0$ $\mathrm{Hz}, 1 \mathrm{H}), 7.52(\mathrm{~d}, J=4.0 \mathrm{~Hz}, 1 \mathrm{H}), 7.30(\mathrm{t}, J=4.0 \mathrm{~Hz}, 1 \mathrm{H}), 7.18-7.12(\mathrm{~m}, 1 \mathrm{H}), 0.92(\mathrm{~d}, J=$ $8.0 \mathrm{~Hz}, 3 \mathrm{H}) .{ }^{19} \mathrm{~F}$ NMR $\left(376 \mathrm{MHz}, \mathrm{CDCl}_{3}\right): \delta-126.0(\mathrm{t}, J=18.8 \mathrm{~Hz}),-137.7--137.8(\mathrm{~m}),-$ 150.7 (tt, $J=19.6,6.8 \mathrm{~Hz}),-154.6(\mathrm{t}, J=21.4 \mathrm{~Hz}),-158.8(\mathrm{q}, J=6.8 \mathrm{~Hz}) .{ }^{13} \mathrm{C}$ NMR $(101$ $\left.\mathrm{MHz}, \mathrm{CDCl}_{3}\right): \delta 150.9\left(\mathrm{dm}, \mathrm{C}-\mathrm{F},{ }^{1} \mathrm{~J}_{\mathrm{C}-\mathrm{F}}=243.7 \mathrm{~Hz}\right), 147.7$ (ddd, C-F, ${ }^{1} \mathrm{~J}_{\mathrm{C}-\mathrm{F}}=251.6 \mathrm{~Hz},{ }^{2} \mathrm{~J}_{\mathrm{C}}$ $\left.\mathrm{F}=9.9 \mathrm{~Hz},{ }^{3} \mathrm{~J}_{\mathrm{C}-\mathrm{F}}=3.1 \mathrm{~Hz}\right), 142.6\left(\mathrm{dm}, \mathrm{C}-\mathrm{F},{ }^{1} \mathrm{~J}_{\mathrm{C}-\mathrm{F}}=258.8 \mathrm{~Hz}\right), 140.5\left(\mathrm{dm}, \mathrm{C}-\mathrm{F},{ }^{1} \mathrm{~J}_{\mathrm{C}-\mathrm{F}}=\right.$ $242.9 \mathrm{~Hz}), 137.65,133.7,130.8\left(\mathrm{~d}, \mathrm{C}-\mathrm{F},{ }^{2} \mathrm{~J}_{\mathrm{C}-\mathrm{F}}=19.1 \mathrm{~Hz}\right), 128.8,117.6-117.1(\mathrm{~m}), 115.7$ $-115.4(\mathrm{~m}),-1.0\left(\mathrm{dd}, \mathrm{C}-\mathrm{F},{ }^{2} \mathrm{~J}_{\mathrm{C}-\mathrm{F}}=14.5 \mathrm{~Hz},{ }^{4} \mathrm{~J}_{\mathrm{C}-\mathrm{F}}=1.5 \mathrm{~Hz}\right) . \mathrm{IR}$ (thin film): 1451, 1089, 1007 , 784, 712. HRMS (EI-TOF) calcd. for $\left[\mathrm{C}_{11} \mathrm{H}_{7} \mathrm{~F}_{5} \mathrm{SSi}\right]\left([\mathrm{M}]^{+}\right)$: 293.9958, found: 293.9961.<smiles>Cn1ccc2cc([Si](C)(c3cc(F)c(F)c(F)c3F)c3ccc4c(ccn4C)c3F)ccc21</smiles>

5-(Fluoro(methyl)(2,3,4,5-tetrafluorophenyl)silyl)-1-methyl-1 $H$-indole (3e). The general procedure was followed with Ru complex 1b (2.7 mg, $5.0 \mu \mathrm{mol}, 2.5 \mathrm{~mol} \%)$, hydrosilane $2 \mathrm{~m}(68.2 \mathrm{mg}, 0.20 \mathrm{mmol})$, and THF $(0.20 \mathrm{~mL})$ at $120^{\circ} \mathrm{C}$ for $24 \mathrm{~h}$. The hydrodefluorination product was afforded as a colorless liquid (63.6 mg, 93\% yield). ${ }^{1} \mathrm{H} \mathrm{NMR}\left(400 \mathrm{MHz}, \mathrm{CDCl}_{3}\right)$ : $\delta 7.95$ (s, 1H), $7.47-7.42(\mathrm{~m}, 2 \mathrm{H}), 7.13-7.08(\mathrm{~m}, 2 \mathrm{H}), 6.57$ (s, 1H), $3.83(\mathrm{~s}, 3 \mathrm{H}), 0.91$ (d, $J=8.0 \mathrm{~Hz}, 3 \mathrm{H}) .{ }^{19} \mathrm{~F} \mathrm{NMR}\left(376 \mathrm{MHz}, \mathrm{CDCl}_{3}\right): \delta-127.2(\mathrm{t}, J=18.4 \mathrm{~Hz}),-138.4--138.6$ (m), -152.0 (tt, $J=18.8,7.5 \mathrm{~Hz}),-155.2(\mathrm{t}, J=21.1 \mathrm{~Hz}),-164.2(\mathrm{q}, J=6.8 \mathrm{~Hz}) .{ }^{13} \mathrm{C}$ NMR 
$\left(101 \mathrm{MHz}, \mathrm{CDCl}_{3}\right): \delta 150.8\left(\mathrm{dm}, \mathrm{C}-\mathrm{F},{ }^{1} \mathrm{~J}_{\mathrm{C}-\mathrm{F}}=243.8 \mathrm{~Hz}\right), 147.6\left(\mathrm{ddd}, \mathrm{C}-\mathrm{F},{ }^{1} \mathrm{~J}_{\mathrm{C}-\mathrm{F}}=251.0\right.$ $\left.\mathrm{Hz},{ }^{2} \mathrm{~J}_{\mathrm{C}-\mathrm{F}}=9.8 \mathrm{~Hz},{ }^{3} \mathrm{~J}_{\mathrm{C}-\mathrm{F}}=3.2 \mathrm{~Hz}\right), 142.2\left(\mathrm{dm}, \mathrm{C}-\mathrm{F},{ }^{1} \mathrm{~J}_{\mathrm{C}-\mathrm{F}}=257.7 \mathrm{~Hz}\right), 140.5(\mathrm{dm}, \mathrm{C}-\mathrm{F}$, $\left.{ }^{1} J_{\mathrm{C}-\mathrm{F}}=257.0 \mathrm{~Hz}\right), 138.4,129.8,128.6,127.7\left(\mathrm{~d}, \mathrm{C}-\mathrm{F},{ }^{4} \mathrm{~J}_{\mathrm{C}-\mathrm{F}}=1.9 \mathrm{~Hz}\right), 126.0,121.0(\mathrm{~d}, \mathrm{C}-$ $\left.\mathrm{F},{ }^{2} \mathrm{~J}_{\mathrm{C}-\mathrm{F}}=16.3 \mathrm{~Hz}\right), 119.0-118.5(\mathrm{~m}), 115.9-115.5(\mathrm{~m}), 109.7,101.7,32.9,-1.6(\mathrm{dd}, \mathrm{C}-$ $\mathrm{F},{ }^{2} J_{\mathrm{C}-\mathrm{F}}=14.4 \mathrm{~Hz},{ }^{4} \mathrm{~J}_{\mathrm{C}-\mathrm{F}}=1.5 \mathrm{~Hz}$ ). IR (thin film): 1515, 1453, 1325, 1091, 1009, 794. HRMS (EI-TOF) calcd. for [ $\left.\mathrm{C}_{16} \mathrm{H}_{12} \mathrm{~F}_{5} \mathrm{NSi}\right]\left([\mathrm{M}]^{+}\right)$: 341.0659, found: 341.0661 .<smiles>C[Si](C)(c1ccccc1OC(F)(F)F)c1cc(F)c(F)c(F)c1F</smiles>

Fluoro(methyl)(2,3,4,5-tetrafluorophenyl)(2-(trifluoromethoxy)phenyl)silane (3f). The general procedure was followed with Ru complex 1b (1.3 mg, $2.5 \mu \mathrm{mol}, 2.5 \mathrm{~mol} \%)$ and hydrosilane $2 \mathrm{~d}(37.2 \mathrm{mg}, 0.10 \mathrm{mmol})$ at $120^{\circ} \mathrm{C}$ for $24 \mathrm{~h}$. The hydrodefluorination product was afforded as a colorless liquid (34.2 mg, 92\% yield). ${ }^{1} \mathrm{H} \mathrm{NMR}\left(400 \mathrm{MHz}, \mathrm{CDCl}_{3}\right): \delta 7.64$ (d, $J=8.0 \mathrm{~Hz}, 1 \mathrm{H}$ ), 7.56 (t, $J=8.0 \mathrm{~Hz}, 1 \mathrm{H}), 7.37$ (t, $J=8.0 \mathrm{~Hz}, 1 \mathrm{H}), 7.29$ (d, J = 8.0 Hz, $1 \mathrm{H}), 7.09-7.03(\mathrm{~m}, 1 \mathrm{H}), 0.88(\mathrm{~d}, J=8.0 \mathrm{~Hz}, 3 \mathrm{H}) .{ }^{19} \mathrm{~F} \mathrm{NMR}\left(376 \mathrm{MHz}, \mathrm{CDCl}_{3}\right): \delta-56.7,-$ $127.2(\mathrm{t}, J=19.2 \mathrm{~Hz}$ ), $-138.0--138.1(\mathrm{~m}),-150.7--150.8(\mathrm{~m}),-154.7--154.8(\mathrm{~m}),-$ 165.8 (q, $J=6.0 \mathrm{~Hz}) .{ }^{13} \mathrm{C}$ NMR (101 MHz, $\left.\mathrm{CDCl}_{3}\right): \delta 154.1-154.1(\mathrm{~m}), 150.9$ (dm, C-F, $\left.{ }^{1} J_{\mathrm{C}-\mathrm{F}}=243.7 \mathrm{~Hz}\right), 147.7$ (ddd, C-F, ${ }^{1} \mathrm{~J}_{\mathrm{C}-\mathrm{F}}=251.5 \mathrm{~Hz},{ }^{2} \mathrm{~J}_{\mathrm{C}-\mathrm{F}}=10.1 \mathrm{~Hz},{ }^{3} \mathrm{~J}_{\mathrm{C}-\mathrm{F}}=3.5 \mathrm{~Hz}$ ), $142.6\left(\mathrm{dm}, \mathrm{C}-\mathrm{F},{ }^{1} \mathrm{~J}_{\mathrm{C}-\mathrm{F}}=258.5 \mathrm{~Hz}\right), 140.4\left(\mathrm{dm}, \mathrm{C}-\mathrm{F},{ }^{1} \mathrm{~J}_{\mathrm{C}-\mathrm{F}}=256.1 \mathrm{~Hz}\right), 135.9\left(\mathrm{~d}, \mathrm{C}-\mathrm{F},{ }^{4} \mathrm{JC}_{\mathrm{C}}\right.$ $\mathrm{F}=2.7 \mathrm{~Hz}), 133.5,126.6,124.6\left(\mathrm{~d}, \mathrm{C}-\mathrm{F},{ }^{2} \mathrm{~J}_{\mathrm{C}-\mathrm{F}}=16.2 \mathrm{~Hz}\right), 120.4\left(\mathrm{q}, \mathrm{C}-\mathrm{F},{ }^{1} J_{\mathrm{C}-\mathrm{F}}=260.1 \mathrm{~Hz}\right)$, $118.3\left(\mathrm{~d}, \mathrm{C}-\mathrm{F},{ }^{4} \mathrm{~J}_{\mathrm{C}-\mathrm{F}}=1.8 \mathrm{~Hz}\right), 117.2-116.6(\mathrm{~m}), 115.7-115.4(\mathrm{~m}),-1.4\left(\mathrm{dd}, \mathrm{C}-\mathrm{F},{ }^{2} \mathrm{~J}_{\mathrm{C}-\mathrm{F}}\right.$ $=14.0 \mathrm{~Hz},{ }^{4} \mathrm{~J}_{\mathrm{C}-\mathrm{F}}=1.9 \mathrm{~Hz}$ ). IR (thin film): 1453, 1246, 1169, 1091, 1010, 767. HRMS (EITOF) calcd. for $\left[\mathrm{C}_{14} \mathrm{H}_{8} \mathrm{~F}_{8} \mathrm{OSi}\right]\left([\mathrm{M}]^{+}\right): 372.0217$, found: 372.0206 .<smiles>C[Si](c1ccc(F)cc1)(c1ccc(F)c(F)c1F)c1cc(F)c(F)c(F)c1F</smiles>

Fluoro(4-fluorophenyl)(methyl)(2,3,4,5-tetrafluorophenyl)silane (3g). The general procedure was followed with Ru complex $\mathbf{1 b}(1.1 \mathrm{mg}, 2.1 \mu \mathrm{mol}, 1.0 \mathrm{~mol} \%)$ and hydrosilane $\mathbf{2 i}$ $(61.2 \mathrm{mg}, 0.20 \mathrm{mmol})$ at $120^{\circ} \mathrm{C}$ for $24 \mathrm{~h}$. The hydrodefluorination product was afforded as 
a colorless liquid (55.0 mg, 90\% yield). ${ }^{1} \mathrm{H}$ NMR (400 MHz, $\left.\mathrm{CDCl}_{3}\right)$ : $\delta 7.63-7.59(\mathrm{~m}, 2 \mathrm{H})$, $7.15(\mathrm{t}, J=8.0 \mathrm{~Hz}, 2 \mathrm{H}), 7.10-7.04(\mathrm{~m}, 1 \mathrm{H}), 0.84(\mathrm{~d}, J=8.0 \mathrm{~Hz}, 3 \mathrm{H}) .{ }^{19} \mathrm{~F} \mathrm{NMR}(376 \mathrm{MHz}$, $\left.\mathrm{CDCl}_{3}\right): \delta-107.5--107.6(\mathrm{~m}),-127.1(\mathrm{t}, J=20.7 \mathrm{~Hz}),-137.7--137.8(\mathrm{~m}),-150.8--$ $150.9(\mathrm{~m}),-154.5(\mathrm{t}, J=21.1 \mathrm{~Hz}),-166.0(\mathrm{q}, J=7.1 \mathrm{~Hz}) .{ }^{13} \mathrm{C} \mathrm{NMR}\left(101 \mathrm{MHz}, \mathrm{CDCl}_{3}\right): \delta$ $165.1\left(\mathrm{~d}, \mathrm{C}-\mathrm{F},{ }^{1} \mathrm{~J}_{\mathrm{C}-\mathrm{F}}=253.1 \mathrm{~Hz}\right), 150.8\left(\mathrm{dm}, \mathrm{C}-\mathrm{F},{ }^{1} \mathrm{~J}_{\mathrm{C}-\mathrm{F}}=242.2 \mathrm{~Hz}\right), 147.8$ (ddd, C-F, ${ }^{1} \mathrm{~J}_{\mathrm{C}}$ $\left.\mathrm{F}=252.0 \mathrm{~Hz},{ }^{2} \mathrm{~J}_{\mathrm{C}-\mathrm{F}}=9.9 \mathrm{~Hz},{ }^{3} \mathrm{~J}_{\mathrm{C}-\mathrm{F}}=3.3 \mathrm{~Hz}\right), 142.5\left(\mathrm{dm}, \mathrm{C}-\mathrm{F},{ }^{1} \mathrm{~J}_{\mathrm{C}-\mathrm{F}}=258.2 \mathrm{~Hz}\right), 140.6(\mathrm{dm}$, $\mathrm{C}-\mathrm{F},{ }^{1} \mathrm{~J}_{\mathrm{C}-\mathrm{F}}=260.0 \mathrm{~Hz}$ ), $136.0\left(\mathrm{ddd}, \mathrm{C}-\mathrm{F},{ }^{3} \mathrm{~J}_{\mathrm{C}-\mathrm{F}}=8.2 \mathrm{~Hz},{ }^{4} \mathrm{~J}_{\mathrm{C}-\mathrm{F}}=1.1 \mathrm{~Hz},{ }^{4} \mathrm{~J}_{\mathrm{C}-\mathrm{F}}=0.8 \mathrm{~Hz}\right.$ ), $128.0\left(\mathrm{dd}, \mathrm{C}-\mathrm{F},{ }^{2} \mathrm{~J}_{\mathrm{C}-\mathrm{F}}=16.7 \mathrm{~Hz},{ }^{3} \mathrm{~J}_{\mathrm{C}-\mathrm{F}}=3.7 \mathrm{~Hz}\right), 117.7-117.2(\mathrm{~m}), 115.9\left(\mathrm{~d}, \mathrm{C}-\mathrm{F},{ }^{2} J_{\mathrm{C}-\mathrm{F}}=\right.$ $20.3 \mathrm{~Hz}$ ), $115.6-115.3(\mathrm{~m}),-1.8\left(\mathrm{dd}, \mathrm{C}-\mathrm{F},{ }^{2} \mathrm{~J}_{\mathrm{C}-\mathrm{F}}=14.0 \mathrm{~Hz},{ }^{4} \mathrm{~J}_{\mathrm{C}-\mathrm{F}}=1.7 \mathrm{~Hz}\right)$. IR (thin film): 1503, 1451, 1324, 1090, 1008, 824, 769. HRMS (El-TOF) calcd. for $\left[\mathrm{C}_{13} \mathrm{H}_{8} \mathrm{~F}_{6} \mathrm{Si}\right]\left([\mathrm{M}]^{+}\right)$: 306.0299, found: 306.0305 .<smiles>Cc1ccc([Si](C)(c2ccc(C)cc2)c2cc(F)c(F)c(F)c2F)cc1</smiles>

Fluoro(methyl)(2,3,4,5-tetrafluorophenyl)(p-tolyl)silane (3h). The general procedure

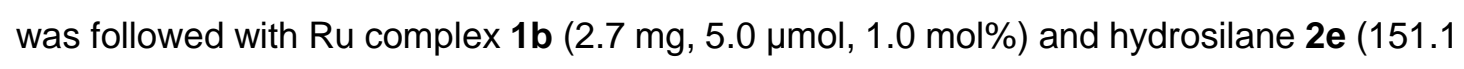
$\mathrm{mg}, 0.50 \mathrm{mmol}$ ) at $120{ }^{\circ} \mathrm{C}$ for $24 \mathrm{~h}$. The hydrodefluorination product was afforded as a colorless liquid (141.4 mg, 94\% yield). ${ }^{1} \mathrm{H}$ NMR (400 MHz, CDCl 3 ): $\delta 7.58$ (d, J $=4.0 \mathrm{~Hz}$, 2H), $7.33(\mathrm{~d}, J=4.0 \mathrm{~Hz}, 2 \mathrm{H}), 7.17-7.11(\mathrm{~m}, 1 \mathrm{H}), 2.45(\mathrm{~s}, 3 \mathrm{H}), 0.90(\mathrm{~d}, J=12.0 \mathrm{~Hz}, 3 \mathrm{H})$. ${ }^{19} \mathrm{~F} \mathrm{NMR}\left(376 \mathrm{MHz}, \mathrm{CDCl}_{3}\right)$ : $\delta-127.1(\mathrm{t}, J=18.8 \mathrm{~Hz}),-138.1--138.2(\mathrm{~m}),-151.4--$ $151.6(\mathrm{~m}),-154.9(\mathrm{t}, J=21.4 \mathrm{~Hz}),-166.1(\mathrm{q}, J=6.8 \mathrm{~Hz}) .{ }^{13} \mathrm{C} \mathrm{NMR}\left(101 \mathrm{MHz}, \mathrm{CDCl}_{3}\right): \delta$ $150.8\left(\mathrm{dm}, \mathrm{C}-\mathrm{F},{ }^{1} \mathrm{~J}_{\mathrm{C}-\mathrm{F}}=242.8 \mathrm{~Hz}\right.$ ), 147.7 (ddd, C-F, ${ }^{1} \mathrm{~J}_{\mathrm{C}-\mathrm{F}}=251.5 \mathrm{~Hz},{ }^{2} \mathrm{~J}_{\mathrm{C}-\mathrm{F}}=9.9 \mathrm{~Hz},{ }^{3} \mathrm{~J}_{\mathrm{C}}$ $\mathrm{F}=3.2 \mathrm{~Hz}), 142.3\left(\mathrm{dm}, \mathrm{C}-\mathrm{F},{ }^{1} \mathrm{~J}_{\mathrm{C}-\mathrm{F}}=257.8 \mathrm{~Hz}\right), 141.8,140.5\left(\mathrm{dm}, \mathrm{C}-\mathrm{F},{ }^{1} \mathrm{~J}_{\mathrm{C}-\mathrm{F}}=257.7 \mathrm{~Hz}\right)$, $133.7\left(\mathrm{~d}, \mathrm{C}-\mathrm{F},{ }^{4} \mathrm{~J}_{\mathrm{C}-\mathrm{F}}=1.4 \mathrm{~Hz}\right), 129.3,128.7\left(\mathrm{~d}, \mathrm{C}-\mathrm{F},{ }^{2} \mathrm{~J}_{\mathrm{C}-\mathrm{F}}=16.4 \mathrm{~Hz}\right), 118.4-117.8(\mathrm{~m})$, $115.7-115.3(\mathrm{~m}), 21.6,-2.0\left(\mathrm{dd}, \mathrm{C}-\mathrm{F},{ }^{2} J_{\mathrm{C}-\mathrm{F}}=14.2 \mathrm{~Hz},{ }^{4} J_{\mathrm{C}-\mathrm{F}}=1.4 \mathrm{~Hz}\right)$. IR (thin film): 1451 , 1324, 1091, 1008, 868, 730. HRMS (EI-TOF) calcd. for $\left[\mathrm{C}_{14} \mathrm{H}_{11} \mathrm{~F}_{5} \mathrm{Si}\right]\left([\mathrm{M}]^{+}\right): 302.0550$, found: 302.0555 . 
<smiles>COc1ccc([Si](C)(C)c2cc(F)c(F)c(F)c2F)cc1</smiles>

Fluoro(4-methoxyphenyl)(methyl)(2,3,4,5-tetrafluorophenyl)silane (3i). The general procedure was followed with Ru complex $1 \mathrm{~b}(2.2 \mathrm{mg}, 4.1 \mu \mathrm{mol}, 1.0 \mathrm{~mol} \%)$ and hydrosilane $2 \mathrm{~g}(127.3 \mathrm{mg}, 0.40 \mathrm{mmol})$ at $120^{\circ} \mathrm{C}$ for $24 \mathrm{~h}$. The hydrodefluorination product was afforded as a colorless liquid (120.2 mg, 94\% yield). ${ }^{1} \mathrm{H}$ NMR (400 MHz, $\left.\mathrm{CDCl}_{3}\right): \delta 7.58$ (d, $J=8.0$ $\mathrm{Hz}, 2 \mathrm{H}), 7.13-7.07(\mathrm{~m}, 1 \mathrm{H}), 7.00(\mathrm{~d}, J=8.0 \mathrm{~Hz}, 2 \mathrm{H}), 3.86(\mathrm{~s}, 3 \mathrm{H}), 0.85(\mathrm{~d}, J=4.0 \mathrm{~Hz}$, 3H). $\left.{ }^{19} \mathrm{~F} \mathrm{NMR} \mathrm{(376} \mathrm{MHz,} \mathrm{CDCl}_{3}\right): \delta-127.3,-138.2,-151.7,-155.0$ (t, $\left.J=19.6 \mathrm{~Hz}\right),-165.1$. ${ }^{13} \mathrm{C} \mathrm{NMR}\left(101 \mathrm{MHz}, \mathrm{CDCl}_{3}\right): \delta 162.3,150.7\left(\mathrm{dm}, \mathrm{C}-\mathrm{F},{ }^{1} \mathrm{~J}_{\mathrm{C}-\mathrm{F}}=241.6 \mathrm{~Hz}\right), 147.6$ (ddd, C$\left.\mathrm{F},{ }^{1} \mathrm{~J}_{\mathrm{C}-\mathrm{F}}=250.9 \mathrm{~Hz},{ }^{2} \mathrm{~J}_{\mathrm{C}-\mathrm{F}}=9.9 \mathrm{~Hz},{ }^{3} \mathrm{~J}_{\mathrm{C}-\mathrm{F}}=3.1 \mathrm{~Hz}\right), 142.2\left(\mathrm{dm}, \mathrm{C}-\mathrm{F},{ }^{1} \mathrm{~J}_{\mathrm{C}-\mathrm{F}}=257.8 \mathrm{~Hz}\right)$, $140.4\left(\mathrm{dm}, \mathrm{C}-\mathrm{F},{ }^{1} \mathrm{~J}_{\mathrm{C}-\mathrm{F}}=258.4 \mathrm{~Hz}\right), 135.4\left(\mathrm{~d}, \mathrm{C}-\mathrm{F},{ }^{3} \mathrm{~J}_{\mathrm{C}-\mathrm{F}}=4.7 \mathrm{~Hz}\right), 123.0\left(\mathrm{~d}, \mathrm{C}-\mathrm{F},{ }^{2} \mathrm{~J}_{\mathrm{C}-\mathrm{F}}=\right.$ $16.8 \mathrm{~Hz}), 118.4-117.9(\mathrm{~m}), 115.7-115.3(\mathrm{~m}), 114.2,55.1\left(\mathrm{~d}, \mathrm{C}-\mathrm{F},{ }^{3} \mathrm{~J}_{\mathrm{C}-\mathrm{F}}=9.2 \mathrm{~Hz}\right),-2.0$ (d, C-F, ${ }^{2} J_{C-F}=14.5 \mathrm{~Hz}$ ). IR (thin film): 1451, 1122, 1091, 1008, 869, 803. HRMS (El-TOF) calcd. for $\left[\mathrm{C}_{14} \mathrm{H}_{11} \mathrm{~F}_{5} \mathrm{OSi}\right]\left([\mathrm{M}]^{+}\right)$: 318.0499, found: 318.0498 .<smiles>CN(C)c1ccc([Si](C)(F)c2cc(F)c(F)c(F)c2F)cc1</smiles>

4-(Fluoro(methyl)(2,3,4,5-tetrafluorophenyl)silyl)- $N, N$-dimethylaniline (3j). The general procedure was followed with Ru complex $\mathbf{1 b}(1.0 \mathrm{mg}, 1.9 \mu \mathrm{mol}, 1.0 \mathrm{~mol} \%)$ and hydrosilane $2 \mathrm{~h}(62.2 \mathrm{mg}, 0.19 \mathrm{mmol})$ at $120^{\circ} \mathrm{C}$ for $24 \mathrm{~h}$. The hydrodefluorination product was afforded as a colorless liquid (55.8 mg, 90\% yield). ${ }^{1} \mathrm{H}$ NMR $\left(400 \mathrm{MHz}, \mathrm{CDCl}_{3}\right): \delta 7.44$ (d, $J=12.0 \mathrm{~Hz}, 2 \mathrm{H}), 7.08-7.03(\mathrm{~m}, 1 \mathrm{H}), 6.74(\mathrm{~d}, J=8.0 \mathrm{~Hz}, 2 \mathrm{H}), 3.00(\mathrm{~s}, 6 \mathrm{H}), 0.78(\mathrm{~d}, J=$ $8.0 \mathrm{~Hz}, 3 \mathrm{H}) .{ }^{19} \mathrm{~F} \mathrm{NMR}\left(376 \mathrm{MHz}, \mathrm{CDCl}_{3}\right): \delta-127.2(\mathrm{t}, J=18.8 \mathrm{~Hz}),-138.4--138.5(\mathrm{~m}),-$ $152.1--152.1(\mathrm{~m}),-155.2(\mathrm{t}, J=21.1 \mathrm{~Hz}),-163.9(\mathrm{~d}, J=1.5 \mathrm{~Hz}) .{ }^{13} \mathrm{C}$ NMR $(101 \mathrm{MHz}$, $\left.\mathrm{CDCl}_{3}\right): \delta 152.3,150.6\left(\mathrm{dd}, \mathrm{C}-\mathrm{F},{ }^{1} \mathrm{~J}_{\mathrm{C}-\mathrm{F}}=241.5 \mathrm{~Hz},{ }^{3} \mathrm{~J}_{\mathrm{C}-\mathrm{F}}=10.2 \mathrm{~Hz}\right), 147.4$ (ddd, C-F, ${ }^{1} \mathrm{~J}_{\mathrm{C}}$ $\left.\mathrm{F}=250.9 \mathrm{~Hz},{ }^{2} \mathrm{~J}_{\mathrm{C}-\mathrm{F}}=9.8 \mathrm{~Hz},{ }^{3} \mathrm{~J}_{\mathrm{C}-\mathrm{F}}=3.2 \mathrm{~Hz}\right), 142.0\left(\mathrm{dm}, \mathrm{C}-\mathrm{F},{ }^{1} \mathrm{~J}_{\mathrm{C}-\mathrm{F}}=256.9 \mathrm{~Hz}\right), 140.3(\mathrm{dm}$, $\left.\mathrm{C}-\mathrm{F},{ }^{1} \mathrm{~J}_{\mathrm{C}-\mathrm{F}}=258.2 \mathrm{~Hz}\right), 134.9,119.0-118.5(\mathrm{~m}), 116.3\left(\mathrm{~d}, \mathrm{C}-\mathrm{F},{ }^{2} \mathrm{~J}_{\mathrm{C}-\mathrm{F}}=17.1 \mathrm{~Hz}\right), 115.6-$ $115.3(\mathrm{~m}), 111.6,40.0,-2.0$ (dd, C-F, ${ }^{2} J_{\mathrm{C}-\mathrm{F}}=14.7 \mathrm{~Hz},{ }^{4} J_{\mathrm{C}-\mathrm{F}}=1.4 \mathrm{~Hz}$ ). IR (thin film): 2922 , 
1596, 1514, 1449, 1118, 1089, 1006, 792. HRMS (El-TOF) calcd. for $\left[\mathrm{C}_{15} \mathrm{H}_{14} \mathrm{~F}_{5} \mathrm{NSi}\right]\left([\mathrm{M}]^{+}\right)$: 331.0816, found: 331.0804 .<smiles>C[Si](F)(Cc1ccccc1)c1cc(F)c(F)c(F)c1F</smiles>

Benzylfluoro(methyl)(2,3,4,5-tetrafluorophenyl)silane (3k). The general procedure was followed with Ru complex 1b (1.1 mg, $2.1 \mu \mathrm{mol}, 1.0 \mathrm{~mol} \%)$ and hydrosilane $2 \mathrm{r}$ (60.4 mg, $0.20 \mathrm{mmol}$ ) at $120^{\circ} \mathrm{C}$ for $24 \mathrm{~h}$. The hydrodefluorination product was afforded as a colorless liquid (54.2 mg, 90\% yield). ${ }^{1} \mathrm{H}$ NMR (400 MHz, $\left.\mathrm{CDCl}_{3}\right): \delta 7.25$ (t, $\left.J=8.0 \mathrm{~Hz}, 2 \mathrm{H}\right), 7.16$ (t, $J=8.0 \mathrm{~Hz}, 1 \mathrm{H}), 7.07(\mathrm{~d}, J=8.0 \mathrm{~Hz}, 2 \mathrm{H}), 7.08-6.99(\mathrm{~m}, 1 \mathrm{H}), 2.61-2.51(\mathrm{~m}, 2 \mathrm{H}), 0.54(\mathrm{~d}$, $J=8.0 \mathrm{~Hz}, 3 \mathrm{H}) .{ }^{19} \mathrm{~F} \mathrm{NMR}\left(376 \mathrm{MHz}, \mathrm{CDCl}_{3}\right): \delta-128.0(\mathrm{t}, J=18.8 \mathrm{~Hz}),-137.9--138.0$ (m), $-151.2--151.4(\mathrm{~m}),-154.9(\mathrm{t}, J=21.4 \mathrm{~Hz}),-167.0(\mathrm{q}, J=6.8 \mathrm{~Hz}) .{ }^{13} \mathrm{C}$ NMR $(101$ $\left.\mathrm{MHz}, \mathrm{CDCl}_{3}\right): \delta 150.4\left(\mathrm{dm}, \mathrm{C}-\mathrm{F},{ }^{1} \mathrm{~J}_{\mathrm{C}-\mathrm{F}}=241.9 \mathrm{~Hz}\right), 147.7$ (ddd, C-F, ${ }^{1} \mathrm{~J}_{\mathrm{C}-\mathrm{F}}=251.7 \mathrm{~Hz},{ }^{2} \mathrm{~J}_{\mathrm{C}}$ $\left.\mathrm{F}=9.9 \mathrm{~Hz},{ }^{3} \mathrm{~J}_{\mathrm{C}-\mathrm{F}}=3.2 \mathrm{~Hz}\right), 142.2\left(\mathrm{dm}, \mathrm{C}-\mathrm{F},{ }^{1} \mathrm{~J}_{\mathrm{C}-\mathrm{F}}=258.0 \mathrm{~Hz}\right), 140.3\left(\mathrm{dm}, \mathrm{C}-\mathrm{F},{ }^{1} \mathrm{~J}_{\mathrm{C}-\mathrm{F}}=\right.$ $256.9 \mathrm{~Hz}), 135.5,128.8,128.6,125.5,117.7-117.2(\mathrm{~m}), 115.3-114.9(\mathrm{~m}), 25.6(\mathrm{~d}, \mathrm{C}-\mathrm{F}$, $\left.{ }^{2} J_{C-F}=13.0 \mathrm{~Hz}\right),-2.5\left(\mathrm{dd}, \mathrm{C}-\mathrm{F},{ }^{2} J_{\mathrm{C}-\mathrm{F}}=13.8 \mathrm{~Hz},{ }^{4} \mathrm{~J}_{\mathrm{C}-\mathrm{F}}=1.0 \mathrm{~Hz}\right.$ ). IR (thin film): 1452,1325 , 1091, 1009, 877, 787. HRMS (El-TOF) calcd. for [ $\left.\mathrm{C}_{14} \mathrm{H}_{11} \mathrm{~F}_{5} \mathrm{Si}\right]\left([\mathrm{M}]^{+}\right): 302.0550$, found: 302.0548 .<smiles>C[Si](F)(CCc1ccccc1)c1cc(F)c(F)c(F)c1F</smiles>

Fluoro(methyl)(phenethyl)(2,3,4,5-tetrafluorophenyl)silane (3I). The general procedure was followed with Ru complex 1b (1.1 mg, $2.1 \mu \mathrm{mol}, 1.0 \mathrm{~mol} \%)$ and hydrosilane $2 \mathrm{~s}$ $(63.2 \mathrm{mg}, 0.20 \mathrm{mmol})$ at $120^{\circ} \mathrm{C}$ for $24 \mathrm{~h}$. The hydrodefluorination product was afforded as a colorless liquid (54.5 mg, 86\% yield). ${ }^{1} \mathrm{H}$ NMR (400 MHz, $\left.\mathrm{CDCl}_{3}\right): \delta 7.29$ (t, $\mathrm{J}=8.0 \mathrm{~Hz}$, $2 \mathrm{H}), 7.21(\mathrm{t}, J=8.0 \mathrm{~Hz}, 3 \mathrm{H}), 7.10-7.05(\mathrm{~m}, 1 \mathrm{H}), 2.86-2.72(\mathrm{~m}, 2 \mathrm{H}), 1.43-1.37(\mathrm{~m}, 2 \mathrm{H})$, $0.56(\mathrm{~d}, J=8.0 \mathrm{~Hz}, 3 \mathrm{H}) .{ }^{19} \mathrm{~F} \mathrm{NMR}\left(376 \mathrm{MHz}, \mathrm{CDCl}_{3}\right): \delta-128.2(\mathrm{t}, J=18.8 \mathrm{~Hz}),-138.0--$ $138.2(\mathrm{~m}),-151.6--151.8(\mathrm{~m}),-155.0(\mathrm{t}, J=20.7 \mathrm{~Hz}),-168.1(\mathrm{q}, J=6.8 \mathrm{~Hz}) .{ }^{13} \mathrm{C}$ NMR $\left(101 \mathrm{MHz}, \mathrm{CDCl}_{3}\right): \delta 150.3\left(\mathrm{dm}, \mathrm{C}-\mathrm{F},{ }^{1} \mathrm{~J}_{\mathrm{C}-\mathrm{F}}=241.5 \mathrm{~Hz}\right), 147.7$ (ddd, C-F, ${ }^{1} \mathrm{~J}_{\mathrm{C}-\mathrm{F}}=251.1$ 
$\left.\mathrm{Hz},{ }^{2} \mathrm{~J}_{\mathrm{C}-\mathrm{F}}=9.8 \mathrm{~Hz},{ }^{3} \mathrm{~J}_{\mathrm{C}-\mathrm{F}}=3.0 \mathrm{~Hz}\right), 143.0,142.1\left(\mathrm{dm}, \mathrm{C}-\mathrm{F},{ }^{1} \mathrm{~J}_{\mathrm{C}-\mathrm{F}}=257.3 \mathrm{~Hz}\right), 140.3(\mathrm{dm}$, $\left.\mathrm{C}-\mathrm{F},{ }^{1} \mathrm{~J}_{\mathrm{C}-\mathrm{F}}=258.2 \mathrm{~Hz}\right), 128.5,127.9,126.2,118.3-118.0(\mathrm{~m}), 115.1-114.7(\mathrm{~m}), 28.3$, $17.6\left(\mathrm{~d}, \mathrm{C}-\mathrm{F},{ }^{2} \mathrm{~J}_{\mathrm{C}-\mathrm{F}}=13.4 \mathrm{~Hz}\right),-1.8\left(\mathrm{dd}, \mathrm{C}-\mathrm{F},{ }^{2} \mathrm{~J}_{\mathrm{C}-\mathrm{F}}=14.0 \mathrm{~Hz},{ }^{4} \mathrm{~J}_{\mathrm{C}-\mathrm{F}}=1.2 \mathrm{~Hz}\right) . \mathrm{IR}$ (thin film): 1515, 1451, 1324, 1126, 1008, 797, 700. HRMS (El-TOF) calcd. for $\left[\mathrm{C}_{15} \mathrm{H}_{13} \mathrm{~F}_{5} \mathrm{Si}\right]\left([\mathrm{M}]^{+}\right)$: 316.0707, found: 316.0706 .<smiles>CCC[Si](C)(F)c1cc(F)c(F)c(F)c1F</smiles>

Fluoro(methyl)(propyl)(2,3,4,5-tetrafluorophenyl)silane $(3 \mathrm{~m})$. The general procedure was followed with Ru complex 1b (1.1 mg, $2.1 \mu \mathrm{mol}, 1.0 \mathrm{~mol} \%)$ and hydrosilane 2t (50.8 $\mathrm{mg}, 0.20 \mathrm{mmol}$ ) at $120^{\circ} \mathrm{C}$ for $24 \mathrm{~h}$. The hydrodefluorination product was afforded as a colorless liquid (42.3 mg, 83\% yield). ${ }^{1} \mathrm{H}$ NMR $\left(400 \mathrm{MHz}, \mathrm{CDCl}_{3}\right): \delta 7.11-7.05(\mathrm{~m}, 1 \mathrm{H})$, $1.50-1.39(\mathrm{~m}, 2 \mathrm{H}), 1.00-0.94(\mathrm{~m}, 5 \mathrm{H}), 0.52(\mathrm{~d}, J=8.0 \mathrm{~Hz}, 3 \mathrm{H}) .{ }^{19} \mathrm{~F}$ NMR $(376 \mathrm{MHz}$, $\left.\mathrm{CDCl}_{3}\right): \delta-128.4(\mathrm{t}, J=19.6 \mathrm{~Hz}),-138.2--138.3(\mathrm{~m}),-152.0--152.1(\mathrm{~m}),-155.2(\mathrm{t}, J$ $=22.9 \mathrm{~Hz}),-167.7--167.8(\mathrm{~m}) \cdot{ }^{13} \mathrm{C}$ NMR $\left(101 \mathrm{MHz}, \mathrm{CDCl}_{3}\right): \delta 150.2\left(\mathrm{dm}, \mathrm{C}-\mathrm{F},{ }^{1} \mathrm{~J}_{\mathrm{C}-\mathrm{F}}=\right.$ $241.3 \mathrm{~Hz}$ ), 147.6 (ddd, C-F, $\left.{ }^{1} \mathrm{~J}_{\mathrm{C}-\mathrm{F}}=251.5 \mathrm{~Hz},{ }^{2} \mathrm{~J}_{\mathrm{C}-\mathrm{F}}=10.0 \mathrm{~Hz},{ }^{3} \mathrm{~J}_{\mathrm{C}-\mathrm{F}}=3.3 \mathrm{~Hz}\right), 141.9$ (dm, $\left.\mathrm{C}-\mathrm{F},{ }^{1} \mathrm{~J}_{\mathrm{C}-\mathrm{F}}=257.3 \mathrm{~Hz}\right), 140.2\left(\mathrm{dm}, \mathrm{C}-\mathrm{F},{ }^{1} \mathrm{~J}_{\mathrm{C}-\mathrm{F}}=258.6 \mathrm{~Hz}\right), 118.6-118.0(\mathrm{~m}), 115.0-$ $114.6(\mathrm{~m}), 18.1\left(\mathrm{~d}, \mathrm{C}-\mathrm{F},{ }^{2} \mathrm{~J}_{\mathrm{C}-\mathrm{F}}=13.3 \mathrm{~Hz}\right), 17.5,15.8,-2.0\left(\mathrm{dd}, \mathrm{C}-\mathrm{F},{ }^{2} \mathrm{~J}_{\mathrm{C}-\mathrm{F}}=14.2 \mathrm{~Hz},{ }^{4} \mathrm{~J}_{\mathrm{C}-\mathrm{F}}\right.$ $=1.2 \mathrm{~Hz}$ ). IR (thin film): 2962, 1517, 1451, 1090, 1010, 798. HRMS (El-TOF) calcd. for $\left[\mathrm{C}_{10} \mathrm{H}_{11} \mathrm{~F}_{5} \mathrm{Si}\right]\left([\mathrm{M}]^{+}\right): 254.0550$, found: 254.0547 .<smiles>C[Si](C)(F)c1cc(F)c(F)c(F)c1F</smiles>

Fluorodimethyl(2,3,4,5-tetrafluorophenyl)silane (3n).

Method A: The general procedure was followed with Ru complex 1b $(2.2 \mathrm{mg}, 4.2 \mu \mathrm{mol}$, $0.10 \mathrm{~mol} \%)$ and hydrosilane $2 \mathrm{u}(905 \mathrm{mg}, 4.0 \mathrm{mmol})$ at $120^{\circ} \mathrm{C}$ for $24 \mathrm{~h}$. The hydrodefluorination product was afforded as a colorless liquid ( $812 \mathrm{mg}, 90 \%$ yield).

Method B: The general procedure was followed with Ru complex 1b $(21.3 \mathrm{mg}, 4.0 \mu \mathrm{mol}$, 
$0.10 \mathrm{~mol} \%)$ and hydrosilane $2 \mathrm{u}(9.04 \mathrm{~g}, 40 \mathrm{mmol})$ at $120^{\circ} \mathrm{C}$ for $24 \mathrm{~h}$. The hydrodefluorination product was afforded as a colorless liquid (6.3 g, 70\% yield). ${ }^{1} \mathrm{H}$ NMR $(400 \mathrm{MHz}$, $\left.\mathrm{CDCl}_{3}\right): \delta 7.10-7.06(\mathrm{~m}, 1 \mathrm{H}), 0.55(\mathrm{~d}, J=8.0 \mathrm{~Hz}, 6 \mathrm{H}) .{ }^{19} \mathrm{~F} \mathrm{NMR}\left(376 \mathrm{MHz}, \mathrm{CDCl}_{3}\right): \delta-$ 128.8, -138.4, -152.1, -155.3, -162.6. ${ }^{13} \mathrm{C}$ NMR (101 MHz, $\left.\mathrm{CDCl}_{3}\right)$ : $\delta 150.5$ (dm, C-F, $\left.{ }^{1} J_{\mathrm{C}-\mathrm{F}}=242.0 \mathrm{~Hz}\right), 147.7\left(\mathrm{dm}, \mathrm{C}-\mathrm{F},{ }^{1} \mathrm{~J}_{\mathrm{C}-\mathrm{F}}=250.6 \mathrm{~Hz}\right), 142.1\left(\mathrm{dm}, \mathrm{C}-\mathrm{F},{ }^{1} \mathrm{~J}_{\mathrm{C}-\mathrm{F}}=257.4 \mathrm{~Hz}\right)$, $140.4\left(\mathrm{dm}, \mathrm{C}-\mathrm{F},{ }^{1} \mathrm{~J}_{\mathrm{C}-\mathrm{F}}=254.7 \mathrm{~Hz}\right), 119.2-118.7(\mathrm{~m}), 114.9-114.5(\mathrm{~m}),-0.6(\mathrm{dd}, \mathrm{C}-\mathrm{F}$, ${ }^{2} J_{C-F}=14.7 \mathrm{~Hz},{ }^{4} J_{C-F}=1.4 \mathrm{~Hz}$ ). IR (thin film): $1451,1092,1009,865,801$. HRMS (El-TOF) calcd. for $\left[\mathrm{C}_{8} \mathrm{H}_{7} \mathrm{~F}_{5} \mathrm{Si}\right]\left([\mathrm{M}]^{+}\right): 226.0237$, found: 226.0233 .
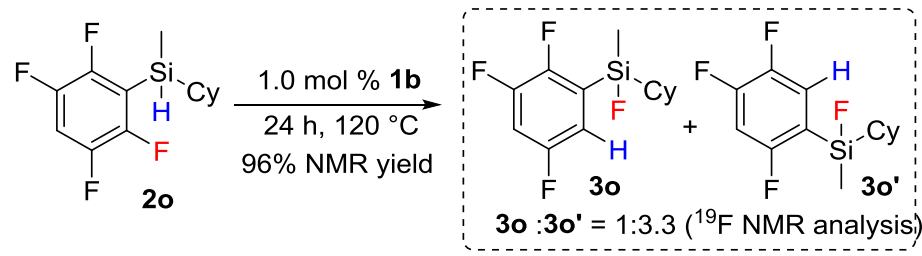

In an argon-filled glovebox, a $5 \mathrm{~mL}$ dried Schlenk tube was charged with Ru complex $1 \mathrm{~b}(0.60 \mathrm{mg}, 1.0 \mathrm{\mu mmol}, 1.0 \mathrm{~mol} \%)$ and hydrosilane 20 (28 $\mathrm{mg}, 0.10 \mathrm{mmol})$. The tube was sealed tightly with a teflon plug and the mixture was stirred at $120^{\circ} \mathrm{C}$ for $24 \mathrm{~h}$. After the resulting reaction mixture was cooled to room temperature, pentafluorobenzene $(17 \mathrm{mg}$, $0.10 \mathrm{mmol}$ ) was added as an internal standard. The yield and ratio of $\mathbf{3 0}: \mathbf{3 0}$ ' were determined by ${ }^{19} \mathrm{~F}$ NMR. 30: ${ }^{19} \mathrm{~F}$ NMR $\left(376 \mathrm{MHz}, \mathrm{CDCl}_{3}\right): \delta-115.0--115.0(\mathrm{~m}),-132.0--$ $132.0(\mathrm{~m}),-133.0--133.0(\mathrm{~m}),-174.5--174.6(\mathrm{~m}) .30$ ': ${ }^{19} \mathrm{~F}$ NMR (376 MHz, $\left.\mathrm{CDCl}_{3}\right): \delta$ $-102.8--102.9(\mathrm{~m}),-130.1--130.1(\mathrm{~m}),-143.4--143.5(\mathrm{~m}),-174.6--174.6(\mathrm{~m})$. 

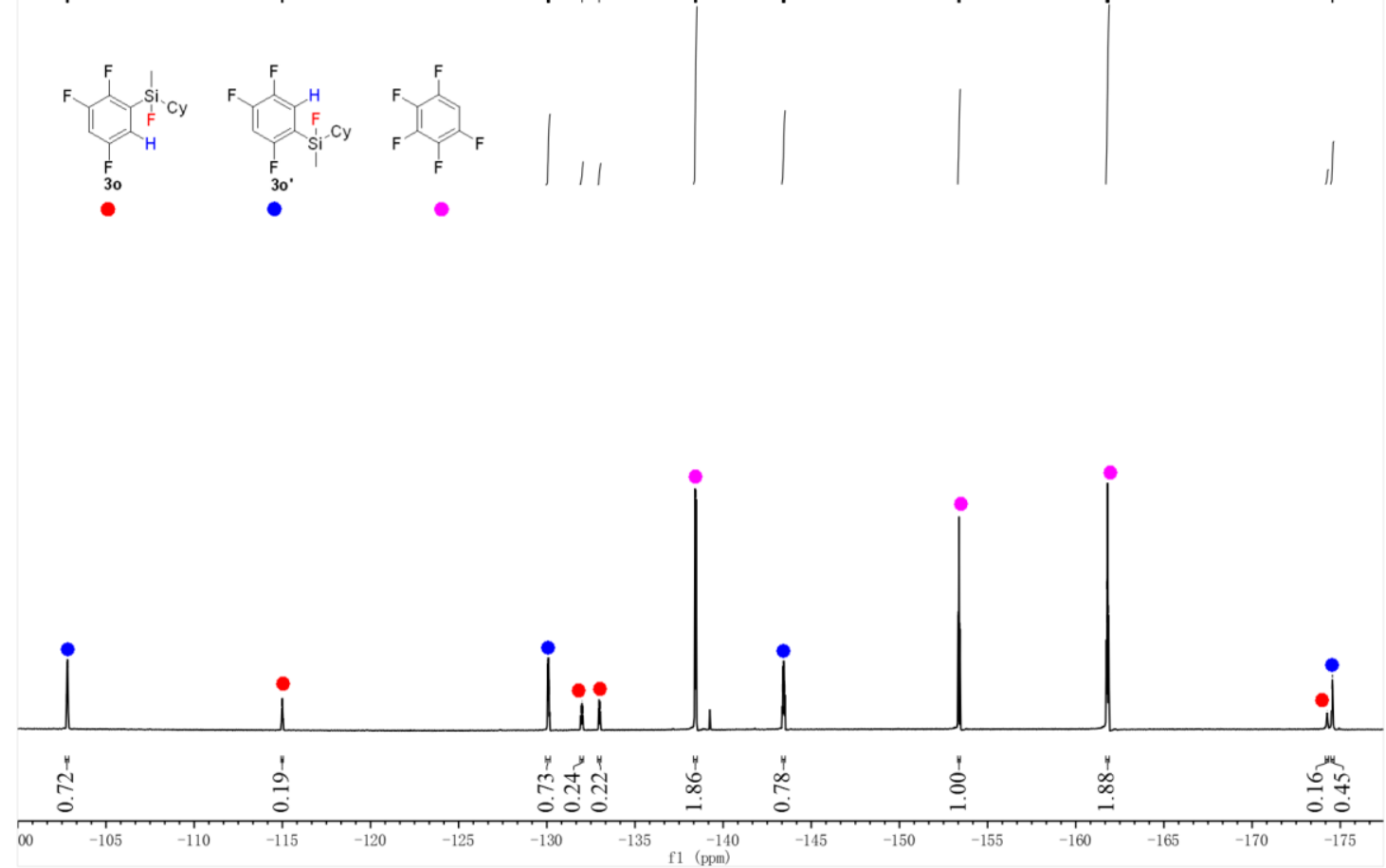

Figure S1. ${ }^{19} \mathrm{~F}$ NMR $\left(376 \mathrm{MHz}, \mathrm{CDCl}_{3}, 298 \mathrm{~K}\right)$ spectrum of the crude mixture of $\mathbf{3 0}, \mathbf{3 0}$, and $\mathrm{C}_{6} \mathrm{~F}_{5} \mathrm{H}$.
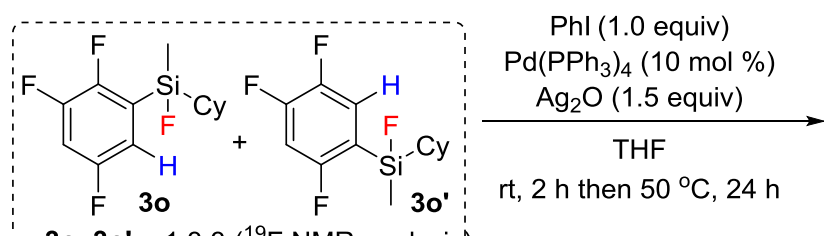

$30: 30^{\prime}=1: 3.3\left({ }^{19} \mathrm{~F}\right.$ NMR analysisi)

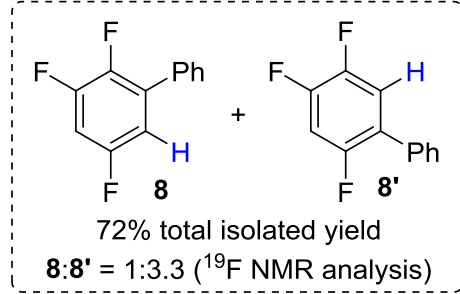

$\mathbf{8}: \mathbf{8}^{\prime}=1: 3.3\left({ }^{19} \mathrm{~F}\right.$ NMR analysis)

In an argon-filled glovebox, a $5 \mathrm{~mL}$ dried Schlenk tube was charged with $\mathrm{Pd}\left(\mathrm{PPh}_{3}\right)_{4}$ (23 mg, $20 \mu \mathrm{mol}, 10 \mathrm{~mol} \%), \mathrm{Ag}_{2} \mathrm{O}(69 \mathrm{mg}, 0.30 \mathrm{mmol})$, and THF (1.0 mL). Then, a mixture of 30 and 3o' (55 mg, $0.20 \mathrm{mmol})$ and $\mathrm{Phl}(41 \mathrm{mg}, 0.20 \mathrm{mmol})$ were added. The tube was sealed tightly with a teflon plug under Ar atmosphere and the mixture was stirred at room temperature for $2 \mathrm{~h}$, and heated at $50^{\circ} \mathrm{C}$ for $24 \mathrm{~h}$. Then the reaction mixture was cooled to room temperature, and filtered through a Celite ${ }^{\circledR}$ plug. The filtrate was collected and the volatiles were removed under reduced pressure. The residue was purified by flash column chromatography on silica gel with petroleum ether as the eluent. The product was obtained as a colorless liquid with a $72 \%$ yield and the ratio of $8: \mathbf{8}^{\prime}$, was determined by ${ }^{19} \mathrm{~F}$ NMR. $\mathbf{8}$ : ${ }^{19} \mathrm{~F}$ NMR (376 MHz, $\mathrm{CDCl}_{3}$ ): $\delta-115.6--115.7(\mathrm{~m}),-133.1$ (dd, $J=20.7,9.4 \mathrm{~Hz}$ ), -148.5 - -148.6 (m). 8': ${ }^{19} \mathrm{~F}$ NMR (376 MHz, $\left.\mathrm{CDCl}_{3}\right)$ : $\delta-119.5--119.5(\mathrm{~m}),-135.1--135.2(\mathrm{~m})$, 
$-142.9--142.9(\mathrm{~m})$. The ${ }^{19} \mathrm{~F}$ NMR was consistent with the spectra of 8 and 8 ' reported in the literature. ${ }^{6}$
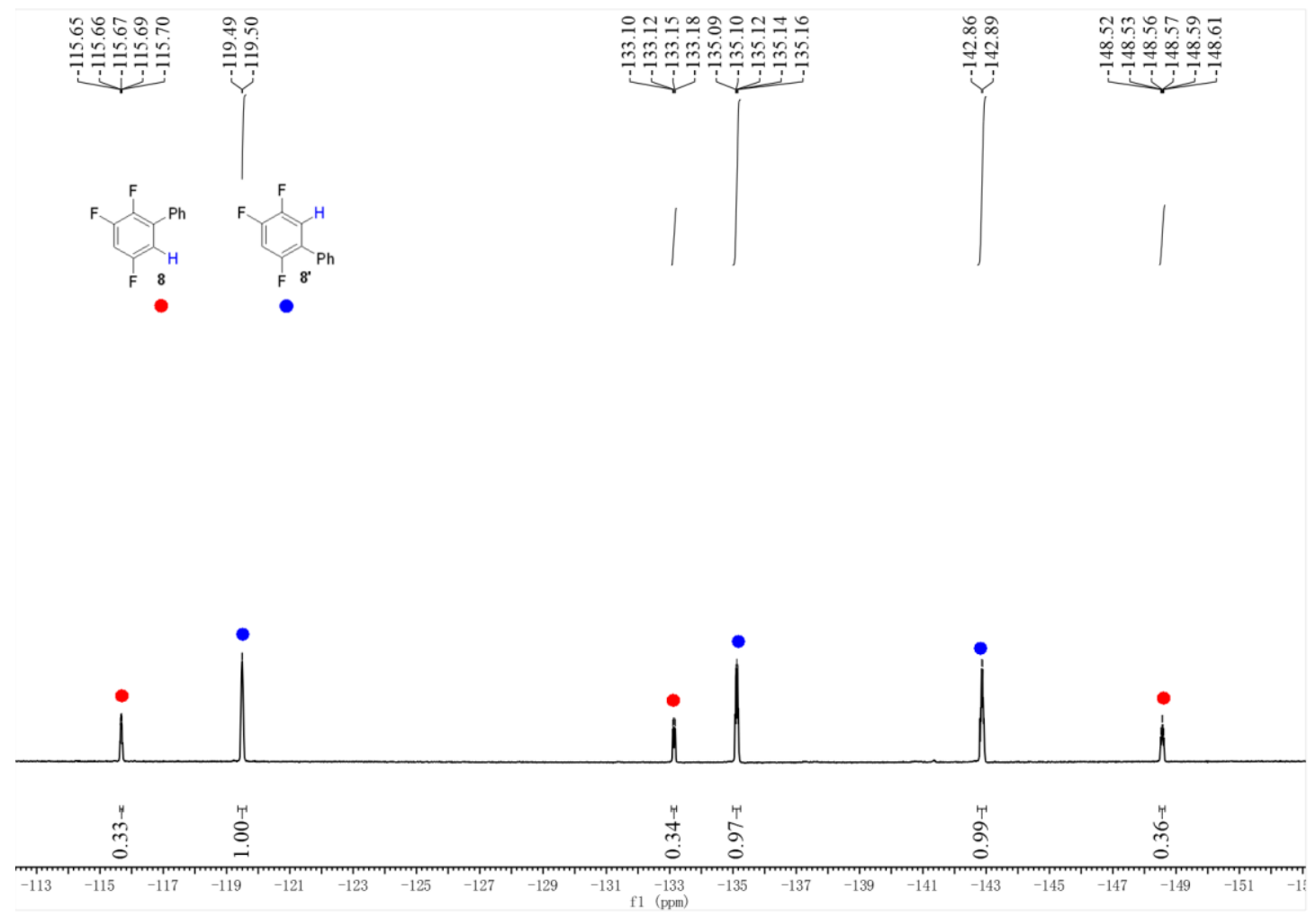

Figure S2. ${ }^{19} \mathrm{~F} \mathrm{NMR}\left(376 \mathrm{MHz}, \mathrm{CDCl}_{3}, 298 \mathrm{~K}\right)$ spectrum of the mixture of 8 and 8 '.

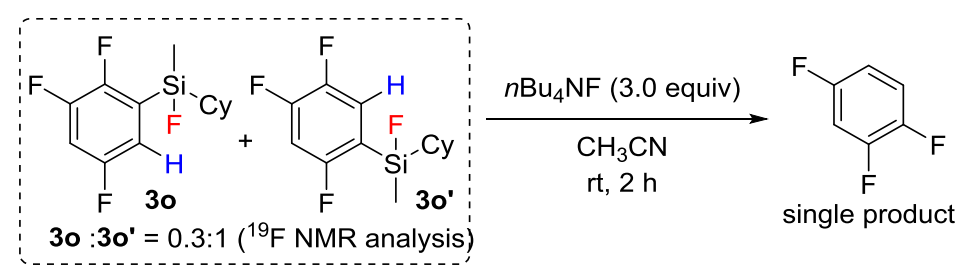

In an argon-filled glovebox, a $5 \mathrm{~mL}$ dried Schlenk tube was charged with a mixture of 30 and 3o' (28 mg, $0.10 \mathrm{mmol})$ and $\mathrm{CH}_{3} \mathrm{CN}(1.0 \mathrm{~mL})$. Then, TBAF $(0.30 \mathrm{~mL}, 0.30 \mathrm{mmol})$ was added. The tube was sealed tightly with a teflon plug under Ar atmosphere and the mixture was stirred at room temperature for $2 \mathrm{~h}$. After that, the mixtures were measured by ${ }^{19} \mathrm{~F}$ NMR. ${ }^{19} \mathrm{~F}$ NMR $\left(376 \mathrm{MHz}, \mathrm{CDCl}_{3}\right): \delta-116.0--116.1(\mathrm{~m}),-134.8--134.8(\mathrm{~m}),-144.4$ $--144.5(\mathrm{~m})$. The ${ }^{19} \mathrm{~F}$ NMR was consistent with the spectra of 1,2,4-trifluorobenzene reported in the literature. ${ }^{7}$ 


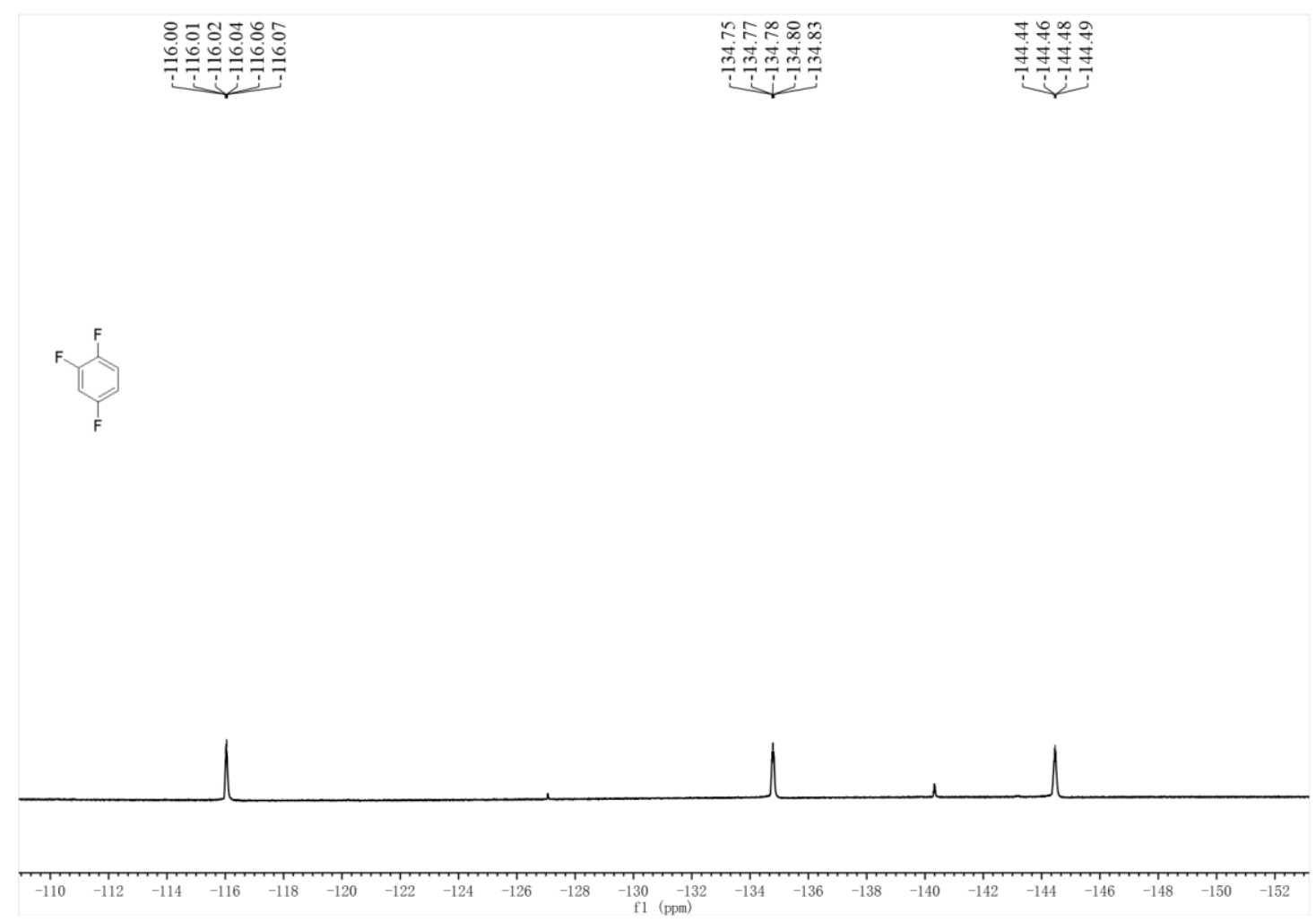

Figure S3. ${ }^{19} \mathrm{~F} \mathrm{NMR}\left(376 \mathrm{MHz}, \mathrm{CDCl}_{3}, 298 \mathrm{~K}\right)$ spectrum of the crude desilylprotonation mixture of 30 and $30^{\prime}$.

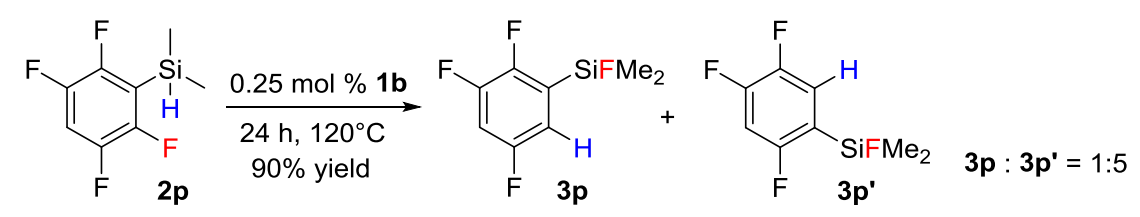

In an argon-filled glovebox, a $5 \mathrm{~mL}$ dried Schlenk tube was charged with Ru complex 1b (1.1 mg, $2.1 \mu \mathrm{mol})$ and hydrosilane $2 \mathbf{p}(172 \mathrm{mg}, 0.83 \mathrm{mmol})$. The tube was sealed tightly with a teflon plug and the mixture was stirred at $120^{\circ} \mathrm{C}$ for $24 \mathrm{~h}$. After the resulting reaction mixture was cooled to room temperature, pentafluorobenzene $(138.9 \mathrm{mg}, 0.83 \mathrm{mmol}, 1.0$ equiv) was added as an internal standard. The yield and ratio of $\mathbf{3 p}: \mathbf{3 p}$ ' were determined by ${ }^{19} \mathrm{~F}$ NMR. 3p: ${ }^{19} \mathrm{~F}$ NMR (376 MHz, $\left.\mathrm{CDCl}_{3}\right)$ : $\delta-114.9--115.0(\mathrm{~m}),-133.1--133.1(\mathrm{~m})$, $-133.3--133.4(\mathrm{~m}),-163.0--163.1(\mathrm{~m}) .3 \mathrm{p}$ : ${ }^{19} \mathrm{~F}$ NMR (376 MHz, $\left.\mathrm{CDCl}_{3}\right): \delta-104.0--$ $104.1(\mathrm{~m}),-129.9--130.0(\mathrm{~m}),-143.4--143.6(\mathrm{~m}),-163.1--163.1(\mathrm{~m})$. 


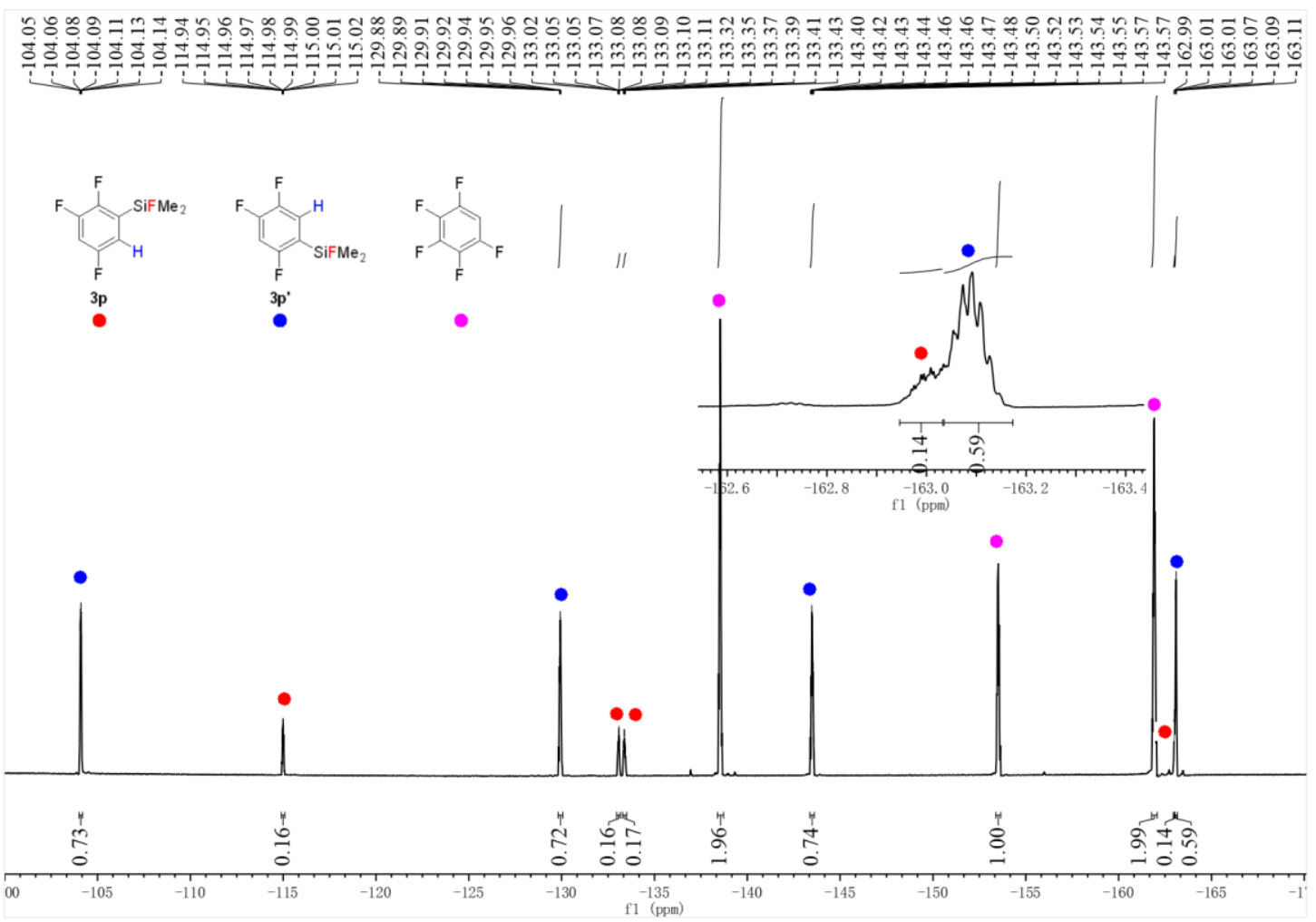

Figure S4. ${ }^{19} \mathrm{~F} \mathrm{NMR} \mathrm{(376} \mathrm{MHz,} \mathrm{CDCl}_{3}, 298 \mathrm{~K}$ ) spectrum of the crude mixture of $\mathbf{3 p}, \mathbf{3} \mathbf{p}$ ', and $\mathrm{C}_{6} \mathrm{~F}_{5} \mathrm{H}$.

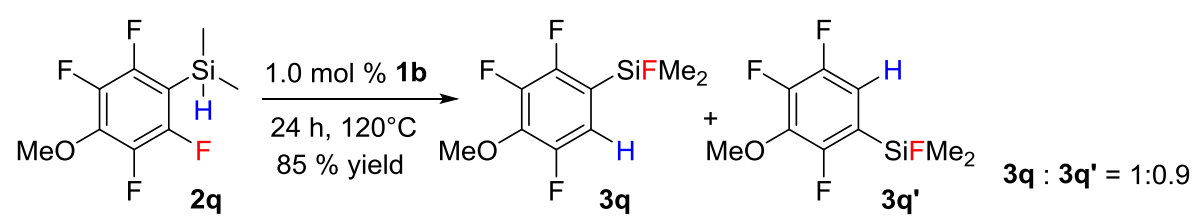

In an argon-filled glovebox, a $5 \mathrm{~mL}$ dried Schlenk tube was charged with Ru complex 1b (1.2 mg, $2.0 \mu \mathrm{mol})$ and hydrosilane 2q (24 mg, $0.10 \mathrm{mmol})$. The tube was sealed tightly with a teflon plug and the mixture was stirred at $120^{\circ} \mathrm{C}$ for $24 \mathrm{~h}$. After the resulting reaction mixture was cooled to room temperature, pentafluorobenzene (17 mg, $0.10 \mathrm{mmol})$ was added as an internal standard. The yield and ratio of $\mathbf{3 q}: \mathbf{3} \mathbf{q}^{\mathbf{\prime}}$ were determined by ${ }^{19} \mathrm{~F}$ NMR. 3q: ${ }^{19} \mathrm{~F} \mathrm{NMR}\left(376 \mathrm{MHz}, \mathrm{CDCl}_{3}\right)$ : $\delta-130.4--130.5(\mathrm{~m}),-147.6--147.6(\mathrm{~m}),-151.4--$ $151.5(\mathrm{~m}),-162.3--162.4(\mathrm{~m}) .3 \mathbf{q}^{\prime}:{ }^{19} \mathrm{~F}$ NMR (376 MHz, $\left.\mathrm{CDCl}_{3}\right)$ : $\delta-122.7--122.7(\mathrm{~m})$, $-133.1--133.1(\mathrm{~m}),-140.2--140.3(\mathrm{~m}),-162.6--162.7(\mathrm{~m})$. 

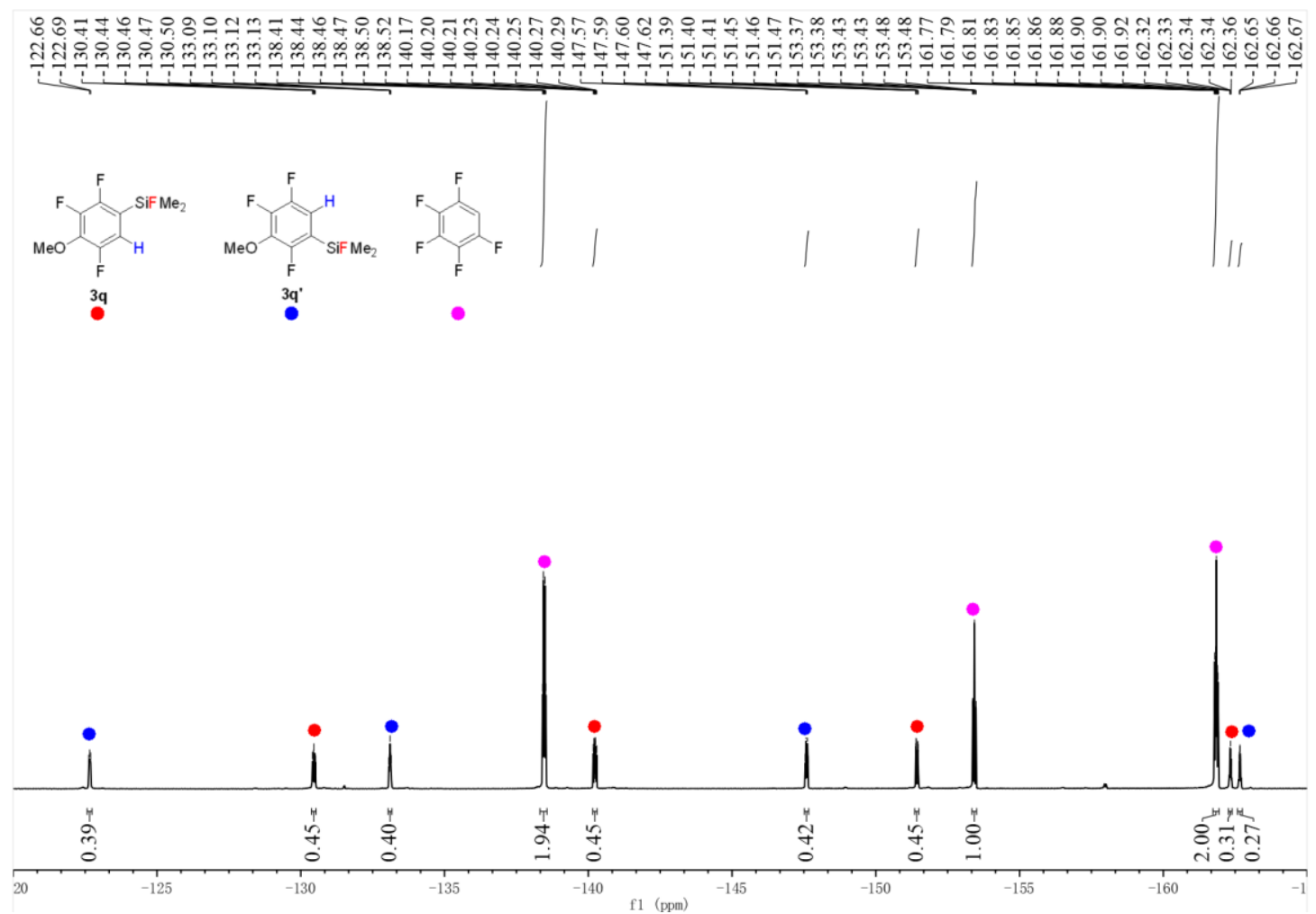

Figure S5. ${ }^{19} \mathrm{~F} \mathrm{NMR} \mathrm{(376} \mathrm{MHz,} \mathrm{CDCl}_{3}, 298 \mathrm{~K}$ ) spectrum of the crude mixture of $\mathbf{3 q}, \mathbf{3 q}$ ', and $\mathrm{C}_{6} \mathrm{~F}_{5} \mathrm{H}$.

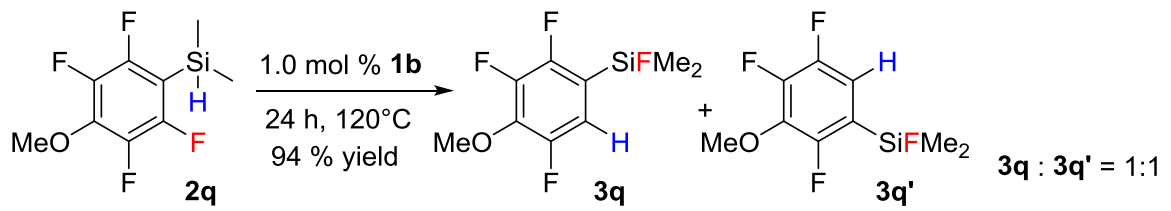

In an argon-filled glovebox, a $5 \mathrm{~mL}$ dried Schlenk tube was charged with Ru complex 1b $(1.2 \mathrm{mg}, 2.0 \mu \mathrm{mol})$ and hydrosilane $2 \mathrm{q}(48 \mathrm{mg}, 0.20 \mathrm{mmol})$. The tube was sealed tightly with a teflon plug and the mixture was stirred at $120^{\circ} \mathrm{C}$ for $24 \mathrm{~h}$. After the resulting reaction mixture was cooled to room temperature, hexafluorobenzene $(18.6 \mathrm{mg}, 0.10 \mathrm{mmol})$ was added as an internal standard. The yield and ratio of $\mathbf{3 q}: \mathbf{3} \mathbf{q}^{\mathbf{\prime}}$ were determined by ${ }^{19} \mathrm{~F}$ NMR. 3q: ${ }^{19} \mathrm{~F} \mathrm{NMR}\left(376 \mathrm{MHz}, \mathrm{CDCl}_{3}\right)$ : $\delta-130.4--130.5(\mathrm{~m}),-147.6--147.6(\mathrm{~m}),-151.4--$ $151.5(\mathrm{~m}),-162.3--162.4(\mathrm{~m}) .3 \mathrm{q}^{\prime}:{ }^{19} \mathrm{~F}$ NMR (376 MHz, $\left.\mathrm{CDCl}_{3}\right)$ : $\delta-122.7--122.7(\mathrm{~m})$, $-133.1--133.1(\mathrm{~m}),-140.2--140.3(\mathrm{~m}),-162.6--162.7(\mathrm{~m})$. 


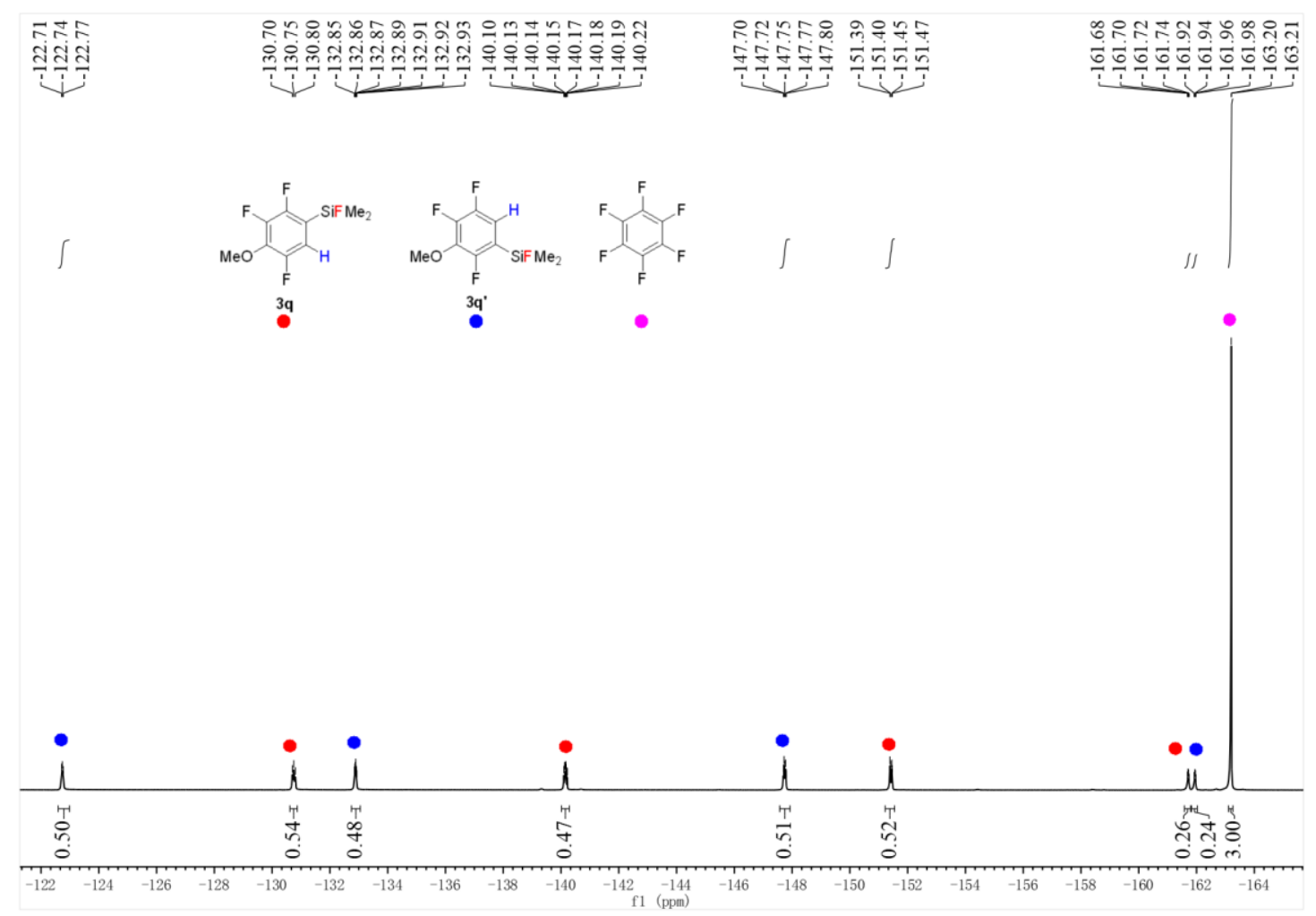

Figure S6. ${ }^{19} \mathrm{~F} \mathrm{NMR} \mathrm{(376} \mathrm{MHz,} \mathrm{CDCl}_{3}, 298 \mathrm{~K}$ ) spectrum of the crude mixture of $\mathbf{3 q}, \mathbf{3 q}$ ', and $\mathrm{C}_{6} \mathrm{~F}_{6}$.

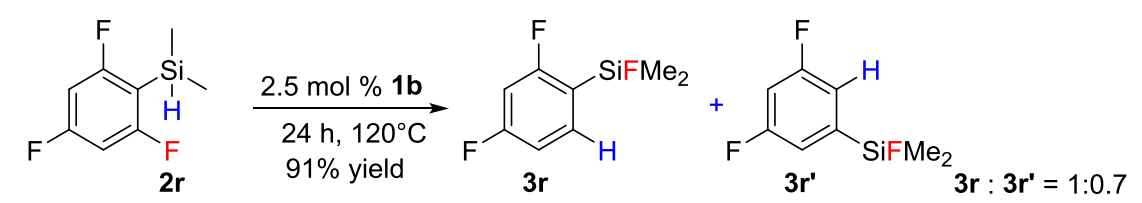

In an argon-filled glovebox, a $5 \mathrm{~mL}$ dried Schlenk tube was charged with Ru complex 1b $(2.7 \mathrm{mg}, 5.0 \mu \mathrm{mol})$ and hydrosilane $2 \mathrm{r}(38 \mathrm{mg}, 0.20 \mathrm{mmol})$. The tube was sealed tightly with a teflon plug and the mixture was stirred at $120^{\circ} \mathrm{C}$ for $24 \mathrm{~h}$. After the resulting reaction mixture was cooled to room temperature, pentafluorobenzene ( $34 \mathrm{mg}, 0.20 \mathrm{mmol}$ ) was added as an internal standard. The yield and ratio of $3 \mathbf{r}: \mathbf{3 r}$ ' were determined by ${ }^{19} \mathrm{~F}$ NMR. 3r: ${ }^{19} \mathrm{~F}$ NMR (376 MHz, $\left.\mathrm{CDCl}_{3}\right)$ : $\delta$-106.5 - -106.6 (m), -109.6 - -109.7 (m), -162.8 - $162.8(\mathrm{~m}) .3 \mathrm{r}$ ': ${ }^{19} \mathrm{~F}$ NMR (376 MHz, $\left.\mathrm{CDCl}_{3}\right): \delta-98.3--98.4(\mathrm{~m}),-162.1--162.2(\mathrm{~m})$. 

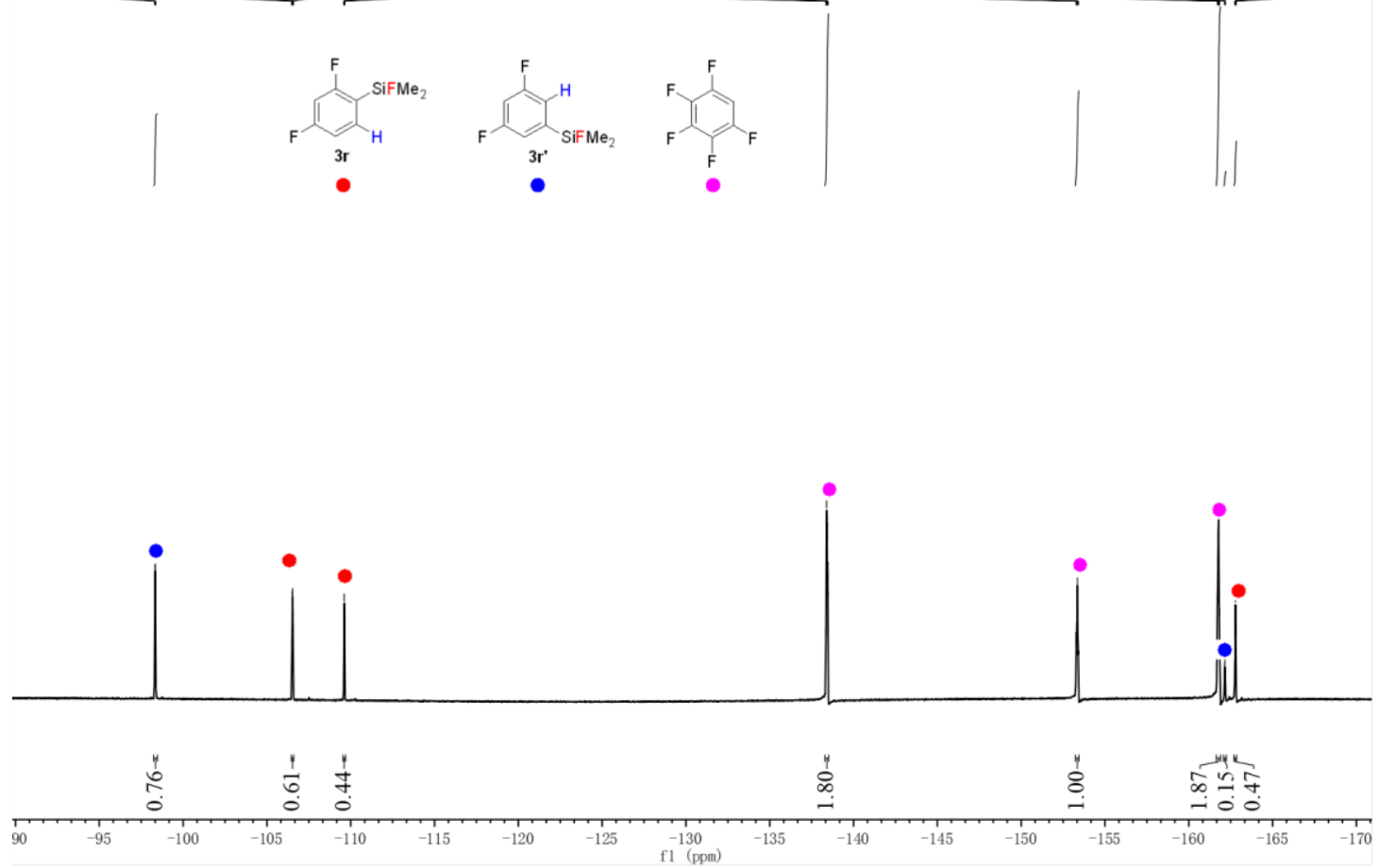

Figure S7. ${ }^{19} \mathrm{~F} \mathrm{NMR}\left(376 \mathrm{MHz}, \mathrm{CDCl}_{3}, 298 \mathrm{~K}\right)$ spectrum of the crude mixture of $\mathbf{3 r}, \mathbf{3 r}$ ', and $\mathrm{C}_{6} \mathrm{~F}_{5} \mathrm{H}$.

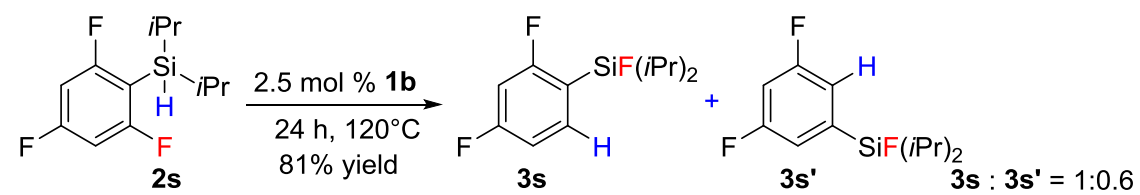

In an argon-filled glovebox, a $5 \mathrm{~mL}$ dried Schlenk tube was charged with Ru complex 1b $(2.7 \mathrm{mg}, 5.0 \mu \mathrm{mol})$ and hydrosilane $2 \mathrm{~s}(49 \mathrm{mg}, 0.20 \mathrm{mmol})$. The tube was sealed tightly with a teflon plug and the mixture was stirred at $120^{\circ} \mathrm{C}$ for $24 \mathrm{~h}$. After the resulting reaction mixture was cooled to room temperature, pentafluorobenzene (17 mg, $0.10 \mathrm{mmol}$ ) was added as an internal standard. The yield and ratio of $\mathbf{3 s}: \mathbf{3} \mathbf{s}^{\prime}$ were determined by ${ }^{19} \mathrm{~F}$ NMR. 3s: ${ }^{19} \mathrm{~F}$ NMR (376 MHz, $\left.\mathrm{CDCl}_{3}\right): \delta-107.1--107.2(\mathrm{~m}),-109.8--109.9(\mathrm{~m}),-187.5(\mathrm{t}, J$ $=6.8 \mathrm{~Hz}) .3 \mathrm{~s}^{\prime}:{ }^{19} \mathrm{~F}$ NMR $\left(376 \mathrm{MHz}, \mathrm{CDCl}_{3}\right): \delta-96.0--96.0(\mathrm{~m}),-186.8--186.8(\mathrm{~m})$. 


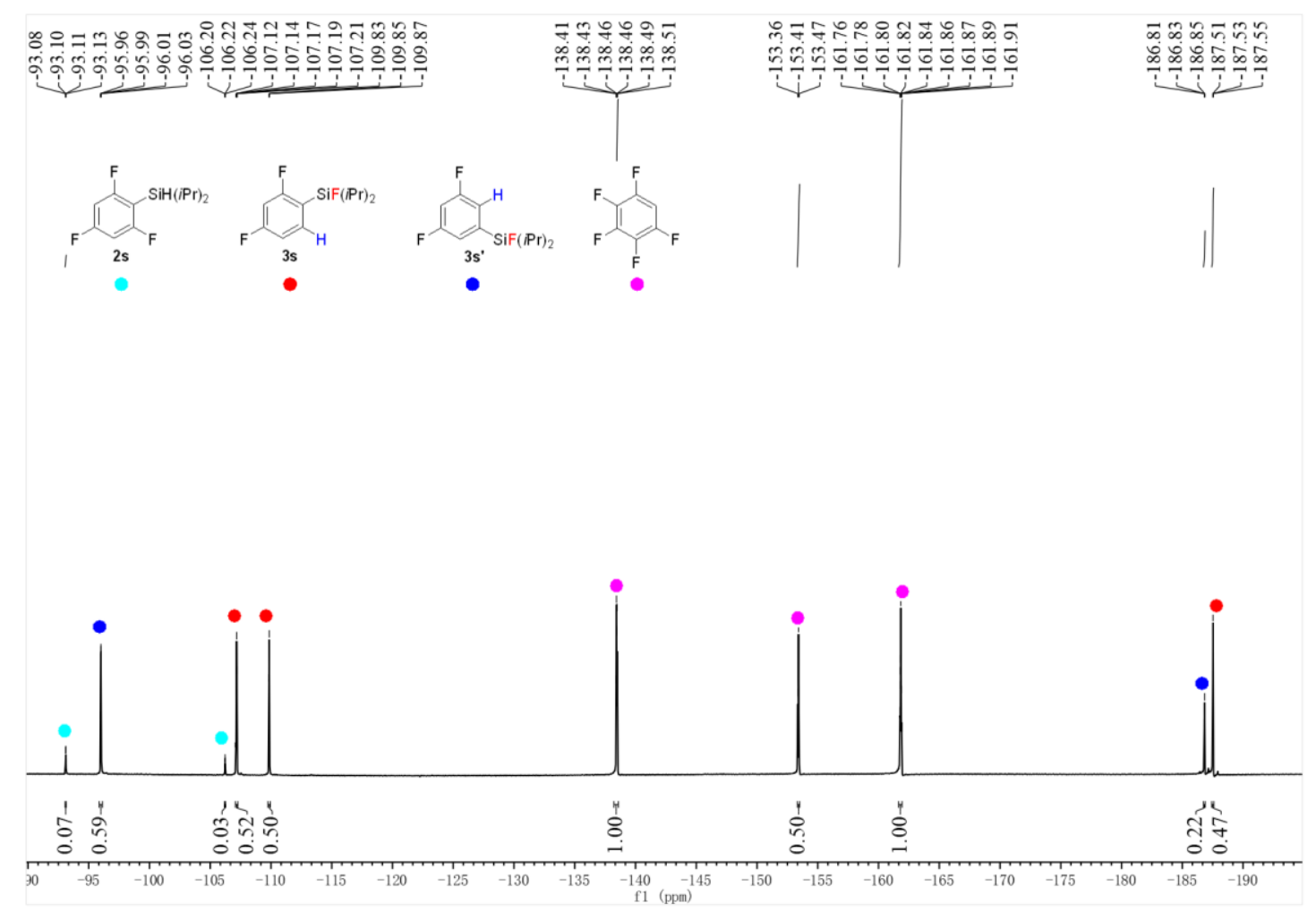

Figure S8. ${ }^{19} \mathrm{~F} \mathrm{NMR}\left(376 \mathrm{MHz}, \mathrm{CDCl}_{3}, 298 \mathrm{~K}\right)$ spectrum of the crude mixture of $3 \mathbf{s}, 3 \mathbf{s}$ ', and $\mathrm{C}_{6} \mathrm{~F}_{5} \mathrm{H}$.
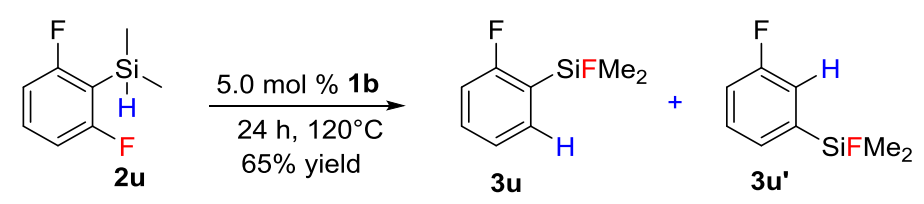

In an argon-filled glovebox, a $5 \mathrm{~mL}$ dried Schlenk tube was charged with Ru complex 1b $(2.7 \mathrm{mg}, 5.0 \mu \mathrm{mol})$ and hydrosilane $2 \mathrm{u}(17.2 \mathrm{mg}, 0.10 \mathrm{mmol})$. The tube was sealed tightly with a teflon plug and the mixture was stirred at $120^{\circ} \mathrm{C}$ for $24 \mathrm{~h}$. After the resulting reaction mixture was cooled to room temperature, pentafluorobenzene $(17 \mathrm{mg}, 0.10 \mathrm{mmol}$ ) was added as an internal standard. The yield and ratio of $3 \mathbf{u}: 3 \mathbf{u}^{\prime}$ were determined by ${ }^{19} \mathrm{~F}$ NMR. 3u: ${ }^{19} \mathrm{~F}$ NMR (376 MHz, $\mathrm{CDCl}_{3}$ ): $\delta-101.4--101.5(\mathrm{~m}),-163.2--163.4(\mathrm{~m}), 3 \mathbf{u}^{\prime}$ : ${ }^{19} \mathrm{~F} \mathrm{NMR}\left(376 \mathrm{MHz}, \mathrm{CDCl}_{3}\right): \delta-113.1$ (td, $\left.J=7.5,3.8 \mathrm{~Hz}\right),-161.9-162.0(\mathrm{~m})$. 

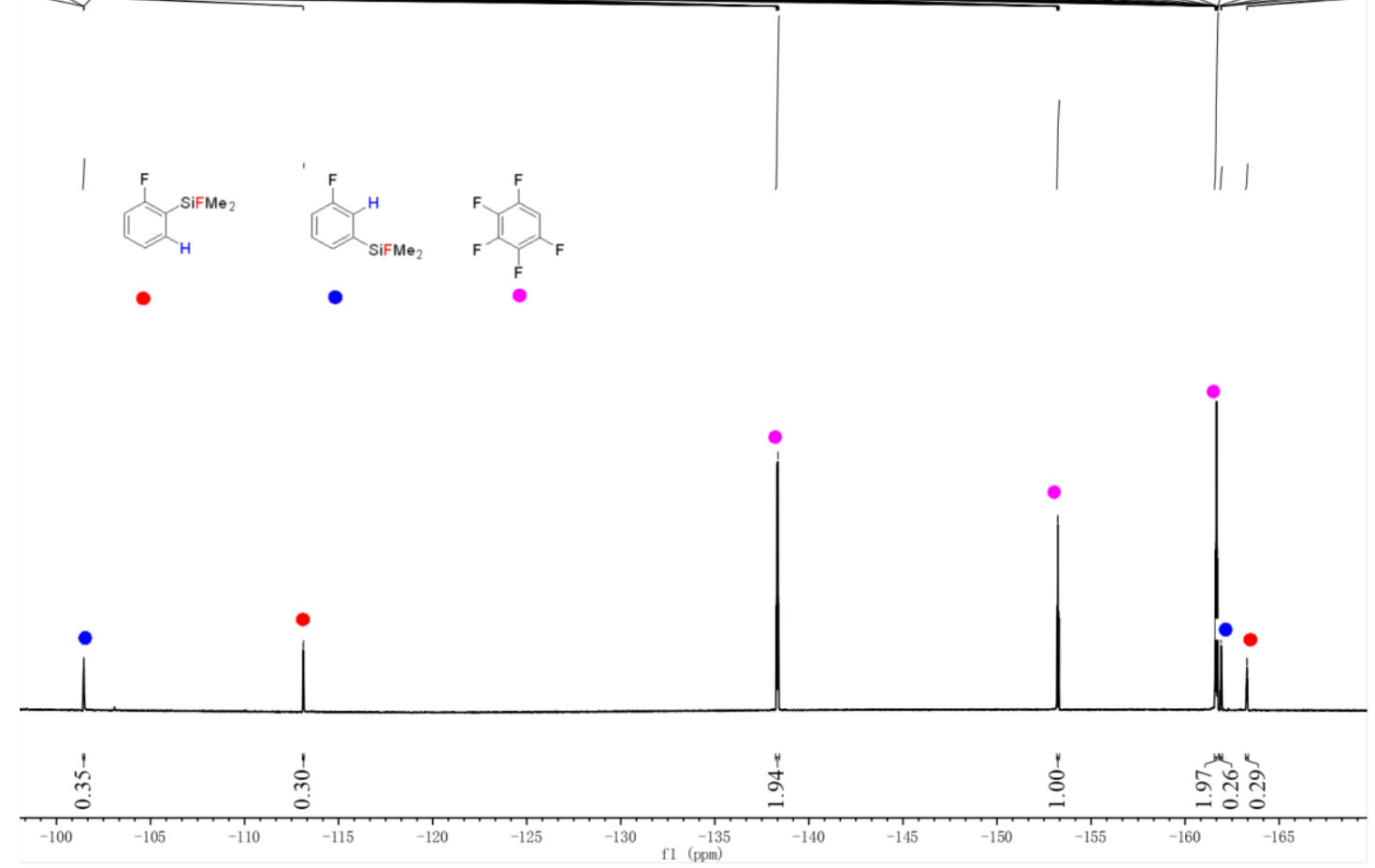

Figure S9. ${ }^{19} \mathrm{~F} \mathrm{NMR}\left(376 \mathrm{MHz}, \mathrm{CDCl}_{3}, 298 \mathrm{~K}\right)$ spectrum of the crude mixture of $\mathbf{3 u}, \mathbf{3} \mathbf{u}^{\text {', }}$ and $\mathrm{C}_{6} \mathrm{~F}_{5} \mathrm{H}$.

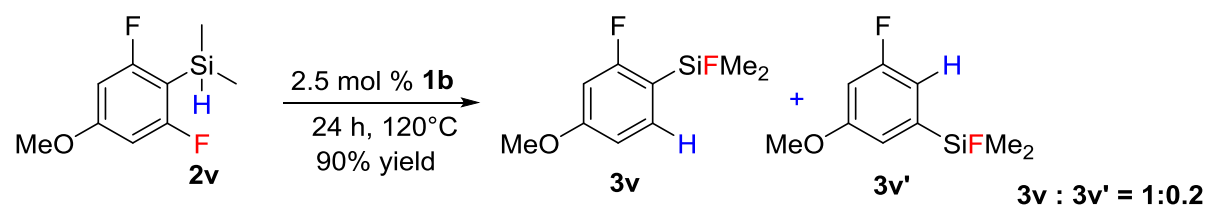

In an argon-filled glovebox, a $5 \mathrm{~mL}$ dried Schlenk tube was charged with Ru complex 1b $(2.7 \mathrm{mg}, 5.0 \mu \mathrm{mol})$ and hydrosilane $2 \mathrm{v}(40.4 \mathrm{mg}, 0.20 \mathrm{mmol})$. The tube was sealed tightly with a teflon plug and the mixture was stirred at $120^{\circ} \mathrm{C}$ for $24 \mathrm{~h}$. After the resulting reaction mixture was cooled to room temperature, pentafluorobenzene (34 $\mathrm{mg}, 0.20 \mathrm{mmol}$ ) was added as an internal standard. The yield and ratio of $3 \mathbf{v}: \mathbf{3} \mathbf{v}$ ' were determined by ${ }^{19} \mathrm{~F}$ NMR. 3v: ${ }^{19} \mathrm{~F}$ NMR (376 MHz, $\mathrm{CDCl}_{3}$ ): $\delta-99.9$ (t, $\left.J=18.8 \mathrm{~Hz}\right),-162.0--162.1(\mathrm{~m}), 3 \mathrm{v}$ ': ${ }^{19} \mathrm{~F} \mathrm{NMR}\left(376 \mathrm{MHz}, \mathrm{CDCl}_{3}\right.$ ): $\delta-111.6$ (dd, $\left.J=11.3,7.5 \mathrm{~Hz}\right),-162.1-162.1(\mathrm{~m})$. 


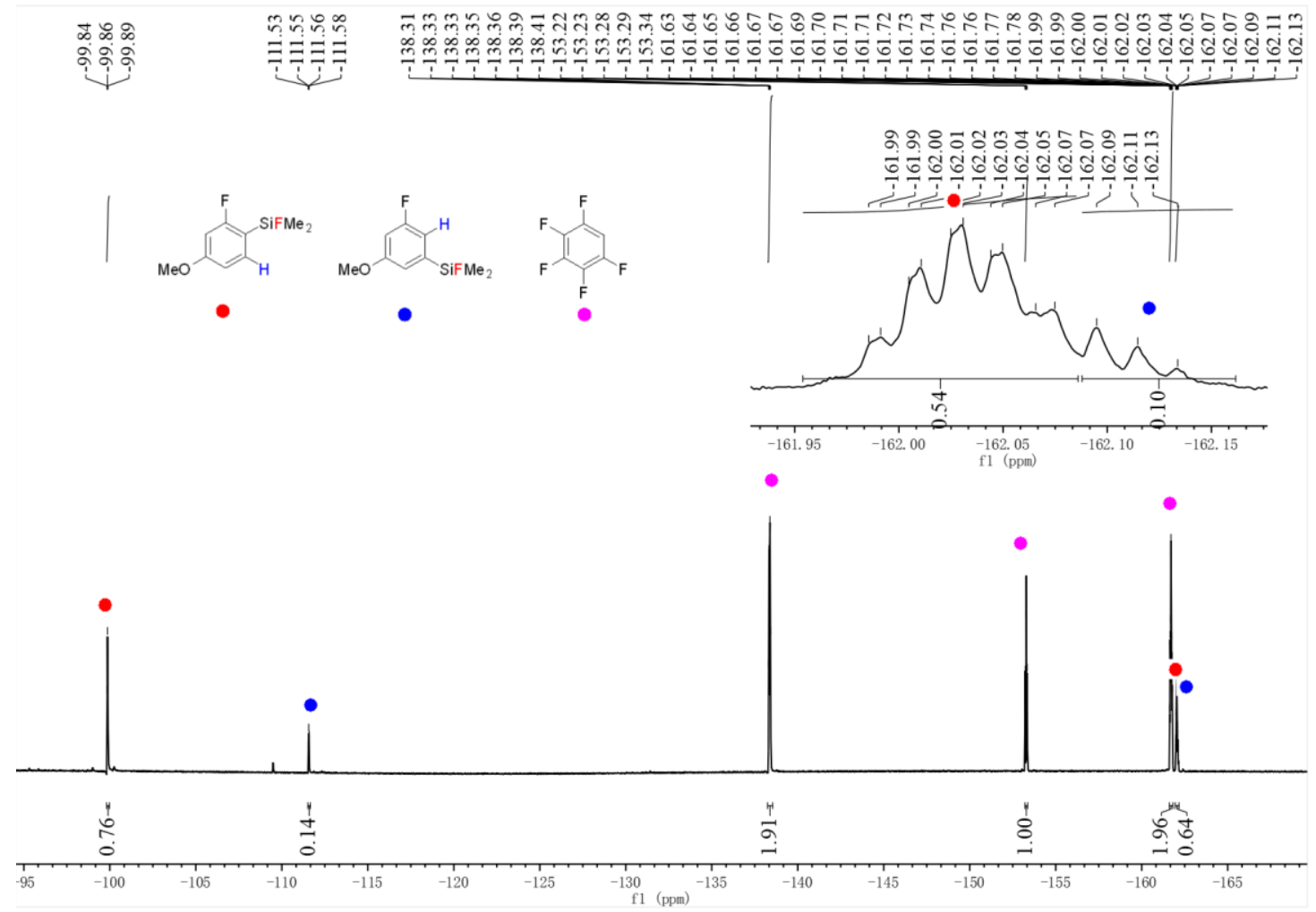

Figure S10. $\left.{ }^{19} \mathrm{~F} \mathrm{NMR} \mathrm{(376} \mathrm{MHz,} \mathrm{CDCl}_{3}, 298 \mathrm{~K}\right)$ spectrum of the crude mixture of $\mathbf{3 v}, \mathbf{3} \mathbf{v}$ ', and $\mathrm{C}_{6} \mathrm{~F}_{5} \mathrm{H}$.

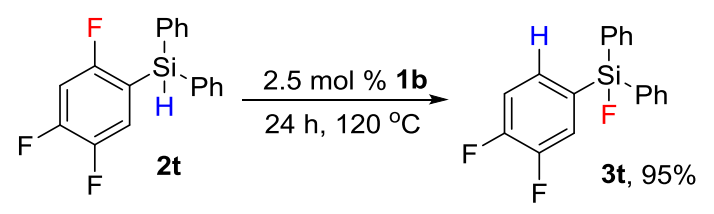

In an argon-filled glovebox, a $5 \mathrm{~mL}$ dried Schlenk tube was charged with Ru complex 1b (3.0 mg, $5.0 \mu \mathrm{mol}, 2.5 \mathrm{~mol} \%)$ and hydrosilane $2 \mathrm{t}(63 \mathrm{mg}, 0.2 \mathrm{mmol})$. The tube was sealed tightly with a teflon plug and the mixture was stirred at $120^{\circ} \mathrm{C}$ for $24 \mathrm{~h}$. After the resulting reaction mixture was cooled to room temperature, the crude mixture was purified by Kugelrohr distillation to obtain the hydrodefluorination product $3 \mathbf{t}$ as colorless liquid (60 mg, 95\% yield). ${ }^{1} \mathrm{H}$ NMR (400 MHz, $\mathrm{CDCl}_{3}$ ): $\delta 7.66$ (d, $J=7.2 \mathrm{~Hz}, 4 \mathrm{H}$ ), 7.54 (t, $J=7.2 \mathrm{~Hz}$, 2H), $7.48-7.43(\mathrm{~m}, 5 \mathrm{H}), 7.39(\mathrm{dd}, J=8.0,5.2 \mathrm{~Hz}, 1 \mathrm{H}), 7.28-7.21(\mathrm{~m}, 1 \mathrm{H}) .{ }^{19} \mathrm{~F}$ NMR $(376$ $\mathrm{MHz}, \mathrm{CDCl}_{3}$ ): $\delta-133.9--134.0(\mathrm{~m}),-137.9$ (ddd, $\left.J=20.7,10.0,7.2 \mathrm{~Hz}\right),-168.8 .{ }^{13} \mathrm{C}$ $\operatorname{NMR}\left(101 \mathrm{MHz}, \mathrm{CDCl}_{3}\right): 152.8\left(\mathrm{dd}, \mathrm{C}-\mathrm{F},{ }^{1} \mathrm{~J}_{\mathrm{C}-\mathrm{F}}=186.5 \mathrm{~Hz},{ }^{2} \mathrm{~J}_{\mathrm{C}-\mathrm{F}}=12.8 \mathrm{~Hz}\right), 150.2(\mathrm{dd}, \mathrm{C}-$ $\left.\mathrm{F},{ }^{1} \mathrm{~J}_{\mathrm{C}-\mathrm{F}}=185.1 \mathrm{~Hz},{ }^{2} \mathrm{~J}_{\mathrm{C}-\mathrm{F}}=12.5 \mathrm{~Hz}\right), 135.0\left(\mathrm{~d}, \mathrm{C}-\mathrm{F},{ }^{4} \mathrm{~J}_{\mathrm{C}-\mathrm{F}}=1.6 \mathrm{~Hz}\right), 131.9-131.8(\mathrm{~m})$, $131.6\left(\mathrm{~d}, \mathrm{C}-\mathrm{F},{ }^{2} \mathrm{~J}_{\mathrm{C}-\mathrm{F}}=17.0 \mathrm{~Hz}\right), 131.3,130.1\left(\mathrm{dm}, \mathrm{C}-\mathrm{F},{ }^{2} J_{\mathrm{C}-\mathrm{F}}=18.3 \mathrm{~Hz}\right), 128.4,123.8(\mathrm{~d}$, $\left.\mathrm{C}-\mathrm{F},{ }^{2} \mathrm{~J}_{\mathrm{C}-\mathrm{F}}=15.5 \mathrm{~Hz}\right), 117.8\left(\mathrm{~d}, \mathrm{C}-\mathrm{F},{ }^{2} \mathrm{~J}_{\mathrm{C}-\mathrm{F}}=16.5 \mathrm{~Hz}\right.$ ). HRMS (El-TOF) calcd. for 
$\left[\mathrm{C}_{18} \mathrm{H}_{13} \mathrm{~F}_{3} \mathrm{Si}\right]\left([\mathrm{M}]^{+}\right): 314.0739$, found: 314.0735 .

$$
\begin{array}{r}
2 a+20 \quad \frac{2.0 \mathrm{~mol} \% \mathbf{1 b}}{\mathrm{THF}, 24 \mathrm{~h}, 120^{\circ} \mathrm{C}} \begin{array}{c}
3 \mathbf{a} \\
90 \%
\end{array} \\
\text { No crossover HDF products observed }
\end{array}
$$

In an argon-filled glovebox, a J. Young NMR tube was charged with Ru complex $\mathbf{1 b}$ (1.2 mg, $2.0 \mu \mathrm{mol}, 2.0 \mathrm{~mol} \%)$, hydrosilane $2 \mathrm{a}(29 \mathrm{mg}, 0.10 \mathrm{mmol}), 20(28 \mathrm{mg}, 0.10 \mathrm{mmol})$, and THF $(0.40 \mathrm{ml})$. The tube was sealed tightly with a teflon plug and the mixture was stirred at $120^{\circ} \mathrm{C}$ for $24 \mathrm{~h}$. After the resulting reaction mixture was cooled to room temperature, pentafluorobenzene $(17 \mathrm{mg}, 0.10 \mathrm{mmol})$ was added as an internal standard and the yields of 3a, 3o, and, 3o' were determined by ${ }^{19} \mathrm{~F}$ NMR.

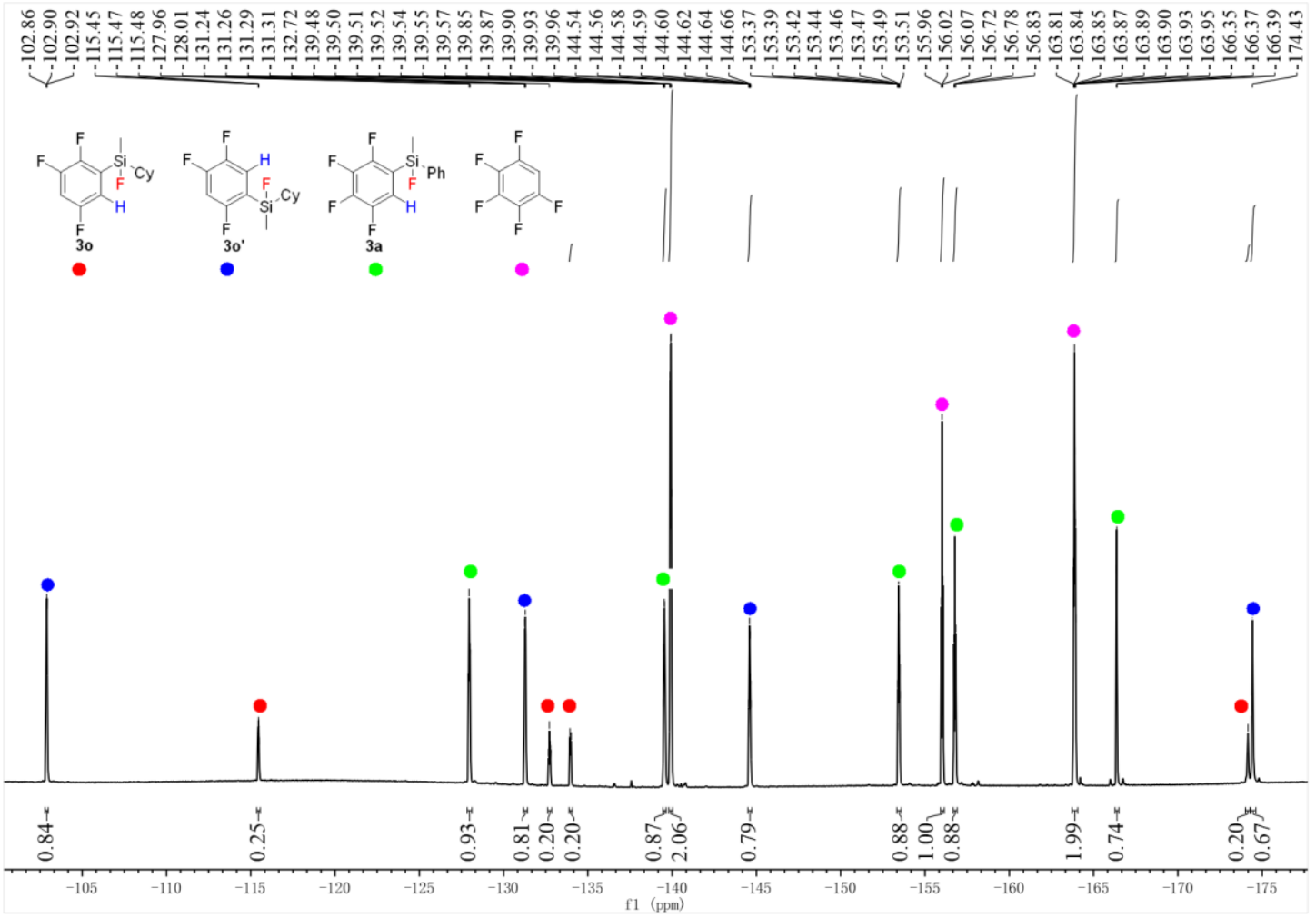

Figure S11. $\left.{ }^{19} \mathrm{~F} \mathrm{NMR} \mathrm{(376} \mathrm{MHz,} \mathrm{CDCl}_{3}, 298 \mathrm{~K}\right)$ spectrum of the crude mixture of $\mathbf{3 a}, \mathbf{3 0}$, 3o', and $\mathrm{C}_{6} \mathrm{~F}_{5} \mathrm{H}$.

The following HDF products $6 \mathbf{a}-\mathbf{6 g}$ could be prepared in high yields by the HDF reactions, but were not included in the table 2 in the manuscript. 
<smiles>Cc1ccccc1[Si](C)(c1ccccc1C)c1cc(F)c(F)c(F)c1F</smiles>

Fluoro(methyl)(2,3,4,5-tetrafluorophenyl)(o-tolyl)silane (6a). The general procedure

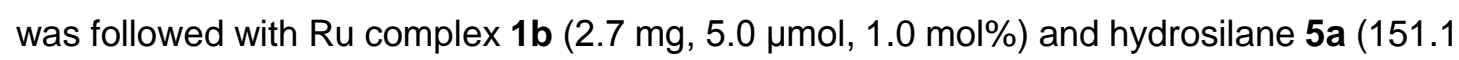
$\mathrm{mg}, 0.50 \mathrm{mmol}$ ) at $120^{\circ} \mathrm{C}$ for $24 \mathrm{~h}$. The hydrodefluorination product was afforded as a colorless liquid (144.4 mg, 96\% yield). ${ }^{1} \mathrm{H}$ NMR (400 MHz, $\left.\mathrm{CDCl}_{3}\right): \delta 7.61$ (d, J = 8.0 Hz, $1 \mathrm{H}), 7.46(\mathrm{t}, J=8.0 \mathrm{~Hz}, 1 \mathrm{H}), 7.33-7.27(\mathrm{~m}, 2 \mathrm{H}), 7.11-7.05(\mathrm{~m}, 1 \mathrm{H}), 2.46(\mathrm{~s}, 3 \mathrm{H}), 0.93$ $(\mathrm{d}, J=8.0 \mathrm{~Hz}, 3 \mathrm{H}) .{ }^{19} \mathrm{~F}$ NMR $\left(376 \mathrm{MHz}, \mathrm{CDCl}_{3}\right): \delta-127.0(\mathrm{t}, J=17.7 \mathrm{~Hz}),-138.0--138.1$ (m), $-151.3--151.4(\mathrm{~m}),-154.8(\mathrm{tm}, J=22.2 \mathrm{~Hz}),-163.1$ (q, $J=1.9 \mathrm{~Hz}) .{ }^{13} \mathrm{C}$ NMR $(101$ $\left.\mathrm{MHz}, \mathrm{CDCl}_{3}\right): \delta 150.8\left(\mathrm{dm}, \mathrm{C}-\mathrm{F},{ }^{1} \mathrm{~J}_{\mathrm{C}-\mathrm{F}}=243.6 \mathrm{~Hz}\right), 147.7\left(\mathrm{ddd}, \mathrm{C}-\mathrm{F},{ }^{1} \mathrm{~J}_{\mathrm{C}-\mathrm{F}}=251.7 \mathrm{~Hz},{ }^{2} \mathrm{~J}_{\mathrm{C}}\right.$ $\left.\mathrm{F}=10.0 \mathrm{~Hz},{ }^{3} \mathrm{~J}_{\mathrm{C}-\mathrm{F}}=3.4 \mathrm{~Hz}\right), 143.9,142.3\left(\mathrm{dm}, \mathrm{C}-\mathrm{F},{ }^{1} \mathrm{~J}_{\mathrm{C}-\mathrm{F}}=258.1 \mathrm{~Hz}\right), 140.5(\mathrm{dm}, \mathrm{C}-\mathrm{F}$, $\left.{ }^{1} J_{\mathrm{C}-\mathrm{F}}=258.7 \mathrm{~Hz}\right), 134.7\left(\mathrm{~d}, \mathrm{C}-\mathrm{F},{ }^{3} \mathrm{~J}_{\mathrm{C}-\mathrm{F}}=4.0 \mathrm{~Hz}\right), 131.7,130.9\left(\mathrm{~d}, \mathrm{C}-\mathrm{F},{ }^{2} \mathrm{~J}_{\mathrm{C}-\mathrm{F}}=14.5 \mathrm{~Hz}\right)$, $130.4,125.5,118.5-118.0(\mathrm{~m}), 115.7-115.3(\mathrm{~m}), 22.6,-1.1$ (dd, C-F, ${ }^{2} \mathrm{~J}_{\mathrm{C}-\mathrm{F}}=14,6 \mathrm{~Hz}$, ${ }^{4} J_{C-F}=1.9 \mathrm{~Hz}$ ). IR (thin film): 1452, 1118, 1009, 871, 782, 766. HRMS (EI-TOF) calcd. for $\left[\mathrm{C}_{14} \mathrm{H}_{11} \mathrm{~F}_{5} \mathrm{Si}\right]\left([\mathrm{M}]^{+}\right):$302.0550, found: 302.0556 .<smiles>C[Si](O)(c1ccc(-c2ccccc2)cc1)c1cc(F)c(F)c(F)c1F</smiles>

[1,1'-Biphenyl]-4-yl(methyl)(2,3,4,5-tetrafluorophenyl)silanol (6b). The general procedure was followed with Ru complex 1b (1.4 mg, $2.5 \mu \mathrm{mol}, 2.5 \mathrm{~mol} \%)$, hydrosilane 5b (36.4 $\mathrm{mg}, 0.10 \mathrm{mmol})$, and THF $(0.20 \mathrm{~mL})$ at $120^{\circ} \mathrm{C}$ for $24 \mathrm{~h}$. The hydrodefluorination product was afforded as a colorless liquid ( $24.8 \mathrm{mg}, 68 \%$ yield). $\mathrm{R}_{f}=0.5$ (petroleum ether / EtOAc $=15: 1) .{ }^{1} \mathrm{H}$ NMR $\left(400 \mathrm{MHz}, \mathrm{CDCl}_{3}\right): \delta 7.69-7.61(\mathrm{~m}, 6 \mathrm{H}), 7.47(\mathrm{t}, J=8.0 \mathrm{~Hz}, 2 \mathrm{H}), 7.39$ (t, $J=8.0 \mathrm{~Hz}, 1 \mathrm{H}), 7.13-7.07(\mathrm{~m}, 1 \mathrm{H}), 2.83(\mathrm{bs}, 1 \mathrm{H}), 0.78$ (s, 3H). ${ }^{19} \mathrm{~F}$ NMR $(376 \mathrm{MHz}$, $\left.\mathrm{CDCl}_{3}\right): \delta-127.5(\mathrm{tm}, J=19.2 \mathrm{~Hz}),-138.4--138.5(\mathrm{~m}),-152.3--152.4(\mathrm{~m}),-155.0--$ $155.2(\mathrm{~m}) .{ }^{13} \mathrm{C}$ NMR $\left(101 \mathrm{MHz}, \mathrm{CDCl}_{3}\right): \delta 150.7$ (dm, C-F, $\left.{ }^{1} \mathrm{~J}_{\mathrm{C}-\mathrm{F}}=241.4 \mathrm{~Hz}\right), 147.5$ (ddd, $\left.\mathrm{C}-\mathrm{F},{ }^{1} \mathrm{~J}_{\mathrm{C}-\mathrm{F}}=251.1 \mathrm{~Hz},{ }^{2} \mathrm{~J}_{\mathrm{C}-\mathrm{F}}=10.0 \mathrm{~Hz},{ }^{3} \mathrm{~J}_{\mathrm{C}-\mathrm{F}}=3.3 \mathrm{~Hz}\right), 143.5,141.6\left(\mathrm{dm}, \mathrm{C}-\mathrm{F},{ }^{1} \mathrm{~J}_{\mathrm{C}-\mathrm{F}}=\right.$ $256.7 \mathrm{~Hz}), 140.7,140.4\left(\mathrm{dm}, \mathrm{C}-\mathrm{F},{ }^{1} J_{\mathrm{C}-\mathrm{F}}=257.9 \mathrm{~Hz}\right), 134.2,133.5,129.0,127.9,127.3$, 
127.0, $120.3-120.0(\mathrm{~m}), 115.8-115.5(\mathrm{~m}),-0.8$. IR (thin film): 3279, 1449, 1323, 1122, 1008, 792, 758. HRMS (El-TOF) calcd. for $\left[\mathrm{C}_{19} \mathrm{H}_{14} \mathrm{~F}_{4} \mathrm{OSi}\right]\left([\mathrm{M}]^{+}\right)$: 362.0750, found: 362.0756 .<smiles>C[Si](O)(c1ccc2ccccc2c1)c1cc(F)c(F)c(F)c1F</smiles>

Methyl(naphthalen-2-yl)(2,3,4,5-tetrafluorophenyl)silanol (6c). The general procedure was followed with Ru complex 1b (1.4 mg, $2.5 \mu \mathrm{mol}, 2.5 \mathrm{~mol} \%)$ and hydrosilane 5c (33.8 $\mathrm{mg}, 0.10 \mathrm{mmol})$, and THF $(0.20 \mathrm{~mL})$ at $120^{\circ} \mathrm{C}$ for $24 \mathrm{~h}$. The hydrodefluorination product was afforded as a colorless liquid $\left(20.1 \mathrm{mg}, 60 \%\right.$ yield). $R_{f}=0.5$ (petroleum ether / EtOAc $=15: 1) .{ }^{1} \mathrm{H}$ NMR $\left(400 \mathrm{MHz}, \mathrm{CDCl}_{3}\right): \delta 8.11(\mathrm{~s}, 1 \mathrm{H}), 7.86(\mathrm{t}, J=8.0 \mathrm{~Hz}, 3 \mathrm{H}), 7.62(\mathrm{~d}, J=$ $8.0 \mathrm{~Hz}, 1 \mathrm{H}), 7.57-7.51(\mathrm{~m}, 2 \mathrm{H}), 7.09-7.03(\mathrm{~m}, 1 \mathrm{H}), 2.89(\mathrm{bs}, 1 \mathrm{H}), 0.82(\mathrm{~s}, 3 \mathrm{H}) .{ }^{19} \mathrm{~F}$ NMR (376 MHz, $\mathrm{CDCl}_{3}$ ): $\delta-132.7--132.9(\mathrm{~m}),-143.7--143.8(\mathrm{~m}),-157.6$ (tdd, $J=19.6,8.3$, $5.6 \mathrm{~Hz}),-160.4--160.5(\mathrm{~m}) .{ }^{13} \mathrm{C}$ NMR $\left(101 \mathrm{MHz}, \mathrm{CDCl}_{3}\right): \delta 150.7$ (dd, C-F, ${ }^{1} \mathrm{~J}_{\mathrm{C}-\mathrm{F}}=240.5$, $8.1 \mathrm{~Hz}), 147.5$ (ddd, C-F, $\left.{ }^{1} \mathrm{~J}_{\mathrm{C}-\mathrm{F}}=251.0 \mathrm{~Hz},{ }^{2} \mathrm{~J}_{\mathrm{C}-\mathrm{F}}=9.9 \mathrm{~Hz},{ }^{3} \mathrm{~J}_{\mathrm{C}-\mathrm{F}}=3.2 \mathrm{~Hz}\right), 141.9(\mathrm{dm}, \mathrm{C}-$ $\mathrm{F},{ }^{1} \mathrm{~J}_{\mathrm{C}-\mathrm{F}}=256.7 \mathrm{~Hz}$ ), $140.3\left(\mathrm{dddd}, \mathrm{C}-\mathrm{F},{ }^{1} \mathrm{~J}_{\mathrm{C}-\mathrm{F}}=258.0 \mathrm{~Hz},{ }^{2} \mathrm{~J}_{\mathrm{C}-\mathrm{F}}=20.5 \mathrm{~Hz},{ }^{3} \mathrm{~J}_{\mathrm{C}-\mathrm{F}}=12.4 \mathrm{~Hz}\right.$, $\left.{ }^{4} J_{C-F}=2.6 \mathrm{~Hz}\right), 135.1,134.5,132.8,132.2,129.1,128.5,127.9,127.8,127.4,126.5,120.2$ $\left(\mathrm{dm}, \mathrm{C}-\mathrm{F},{ }^{2} J_{\mathrm{C}-\mathrm{F}}=29.0 \mathrm{~Hz}\right), 115.9-115.6(\mathrm{~m}),-0.8\left(\mathrm{~d}, \mathrm{C}-\mathrm{F},{ }^{4} J_{\mathrm{C}-\mathrm{F}}=1.4 \mathrm{~Hz}\right)$. IR (thin film): $3284,1514,1448,1321,1088,1007,858,780$. HRMS (El-TOF) calcd. for $\left[\mathrm{C}_{17} \mathrm{H}_{12} \mathrm{~F}_{4} \mathrm{OSi}\right]$ $\left([\mathrm{M}]^{+}\right)$: 336.0594, found: 336.0595 .<smiles>COc1ccc2ccccc2c1[Si](C)(O)c1cc(F)c(F)c(F)c1F</smiles>

(2-Methoxynaphthalen-1-yl)(methyl)(2,3,4,5-tetrafluorophenyl)silanol (6d). The general procedure was followed with Ru complex $1 \mathrm{~b}(2.7 \mathrm{mg}, 5.0 \mu \mathrm{mol}, 2.5 \mathrm{~mol} \%)$, hydrosilane 5d $(73.6 \mathrm{mg}, 0.20 \mathrm{mmol})$, and THF $(0.20 \mathrm{~mL})$ at $120{ }^{\circ} \mathrm{C}$ for $24 \mathrm{~h}$. The hydrodefluorination product was afforded as a colorless liquid $\left(49.8 \mathrm{mg}, 68 \%\right.$ yield). $R_{f}=0.5$ (petroleum ether / EtOAc = $15: 1) .{ }^{1} \mathrm{H}$ NMR $\left(400 \mathrm{MHz}, \mathrm{CDCl}_{3}\right): \delta 8.09(\mathrm{~d}, J=8.0 \mathrm{~Hz}, 1 \mathrm{H}), 7.96(\mathrm{~d}, J=8.0$ $\mathrm{Hz}, 1 \mathrm{H}), 7.80(\mathrm{~d}, J=8.0 \mathrm{~Hz}, 1 \mathrm{H}), 7.41(\mathrm{t}, J=8.0 \mathrm{~Hz}, 1 \mathrm{H}) 7.34(\mathrm{t}, J=8.0 \mathrm{~Hz}, 1 \mathrm{H}), 7.24(\mathrm{~d}$, 
$J=12.0 \mathrm{~Hz}, 1 \mathrm{H}), 6.97-6.92(\mathrm{~m}, 1 \mathrm{H}), 3.87(\mathrm{~s}, 3 \mathrm{H}), 1.24(\mathrm{bs}, 1 \mathrm{H}), 0.91(\mathrm{~s}, 3 \mathrm{H}) .{ }^{19} \mathrm{~F} \mathrm{NMR}$ (376 MHz, $\left.\mathrm{CDCl}_{3}\right): \delta-128.2(\mathrm{t}, J=18.8 \mathrm{~Hz}),-139.2--139.4(\mathrm{~m}),-153.4--153.5(\mathrm{~m}),-$ $155.7(\mathrm{t}, J=21.4 \mathrm{~Hz}) .{ }^{13} \mathrm{C}$ NMR $\left(101 \mathrm{MHz}, \mathrm{CDCl}_{3}\right): \delta 163.4,150.7\left(\mathrm{dm}, \mathrm{C}-\mathrm{F},{ }^{1} \mathrm{~J}_{\mathrm{C}-\mathrm{F}}=242.1\right.$ $\mathrm{Hz}$ ), 147.3 (ddd, C-F, $\left.{ }^{1} \mathrm{~J}_{\mathrm{C}-\mathrm{F}}=250.2 \mathrm{~Hz},{ }^{2} \mathrm{~J}_{\mathrm{C}-\mathrm{F}}=9.9 \mathrm{~Hz},{ }^{3} \mathrm{~J}_{\mathrm{C}-\mathrm{F}}=3.3 \mathrm{~Hz}\right), 141.5,140.2,137.8$, 134.0, 129.6, 129.0, 127.1, 126.7, 124.0, $122.3\left(\mathrm{dm}, \mathrm{C}-\mathrm{F},{ }^{2} \mathrm{~J}_{\mathrm{C}-\mathrm{F}}=28.1 \mathrm{~Hz}\right), 115.6-115.2$ (m), 115.4, 112.8, 56.5, 2.5 (d, C-F, $\left.{ }^{4} J_{C-F}=1.7 \mathrm{~Hz}\right)$. IR (thin film): 3401, 1511, 1450, 1320, 1088, 1007, 785. HRMS (EI-TOF) calcd. for $\left[\mathrm{C}_{18} \mathrm{H}_{14} \mathrm{~F}_{4} \mathrm{O}_{2} \mathrm{Si}\right]\left([\mathrm{M}]^{+}\right)$: 366.0699 , found: 366.0698 .<smiles>C[Si](c1ccc(-n2cccc2)cc1)(c1ccc(-n2cccc2)cc1F)c1cc(F)c(F)c(F)c1F</smiles>

1-(4-(Fluoro(methyl)(2,3,4,5-tetrafluorophenyl)silyl)phenyl)-1 $H$-pyrrole (6e). The general procedure was followed with Ru complex 1b (2.7 mg, $5.0 \mu \mathrm{mol}, 2.5 \mathrm{~mol} \%)$, hydrosilane 5e $(70.6 \mathrm{mg}, 0.20 \mathrm{mmol})$, and THF $(0.20 \mathrm{~mL})$ at $120^{\circ} \mathrm{C}$ for $24 \mathrm{~h}$. The hydrodefluorination product was afforded as a white solid $\left(64.0 \mathrm{mg}, 91 \%\right.$ yield). ${ }^{1} \mathrm{H} \mathrm{NMR}\left(400 \mathrm{MHz}, \mathrm{CDCl}_{3}\right): \delta$ $7.69(\mathrm{~d}, J=8.0 \mathrm{~Hz}, 2 \mathrm{H}), 7.49(\mathrm{~d}, J=8.0 \mathrm{~Hz}, 2 \mathrm{H}), 7.16-7.11(\mathrm{~m}, 3 \mathrm{H}), 6.40(\mathrm{~s}, 2 \mathrm{H}), 0.88$ $(\mathrm{d}, J=8.0 \mathrm{~Hz}, 3 \mathrm{H}) .{ }^{19} \mathrm{~F} \mathrm{NMR}\left(376 \mathrm{MHz}, \mathrm{CDCl}_{3}\right): \delta-127.0(\mathrm{t}, J=18.8 \mathrm{~Hz}),-137.6--137.7$ (m), $-150.7--150.8(\mathrm{~m}),-154.4(\mathrm{t}, J=21.4 \mathrm{~Hz}),-166.1(\mathrm{~d}, J=6.0 \mathrm{~Hz}) .{ }^{13} \mathrm{C}$ NMR $(101$ $\left.\mathrm{MHz}, \mathrm{CDCl}_{3}\right): \delta 150.8\left(\mathrm{dm}, \mathrm{C}-\mathrm{F},{ }^{1} \mathrm{~J}_{\mathrm{C}-\mathrm{F}}=241.5 \mathrm{~Hz}\right), 147.8\left(\mathrm{ddd}, \mathrm{C}-\mathrm{F},{ }^{1} \mathrm{~J}_{\mathrm{C}-\mathrm{F}}=251.7 \mathrm{~Hz},{ }^{2} \mathrm{~J}_{\mathrm{C}}\right.$ $\left.\mathrm{F}=9.7 \mathrm{~Hz},{ }^{3} \mathrm{~J}_{\mathrm{C}-\mathrm{F}}=3.2 \mathrm{~Hz}\right), 143.1,142.5\left(\mathrm{dm}, \mathrm{C}-\mathrm{F},{ }^{1} \mathrm{~J}_{\mathrm{C}-\mathrm{F}}=258.6 \mathrm{~Hz}\right), 140.5\left(\mathrm{dm}, \mathrm{C}-\mathrm{F},{ }^{1} \mathrm{~J}_{\mathrm{C}}\right.$ $\mathrm{F}=261.0 \mathrm{~Hz}), 135.3,128.8\left(\mathrm{~d}, \mathrm{C}-\mathrm{F},{ }^{2} \mathrm{~J}_{\mathrm{C}-\mathrm{F}}=16.8 \mathrm{~Hz}\right), 120.0,119.1,117.8-117.3(\mathrm{~m})$, $115.6-115.3(\mathrm{~m}), 111.3,-1.8\left(\mathrm{dd}, \mathrm{C}-\mathrm{F},{ }^{2} J_{\mathrm{C}-\mathrm{F}}=14.1 \mathrm{~Hz},{ }^{4} \mathrm{~J}_{\mathrm{C}-\mathrm{F}}=1.4 \mathrm{~Hz}\right) . \mathrm{IR}$ (thin film): 1511 , 1448, 1321, 1092, 1004, 868, 781. HRMS (El-TOF) calcd. for $\left[\mathrm{C}_{17} \mathrm{H}_{12} \mathrm{~F}_{5} \mathrm{NSi}\right]\left([\mathrm{M}]^{+}\right)$: 353.0659, found: 353.0663 .<smiles>C[Si](c1ccsc1)(c1ccsc1)c1cc(F)c(F)c(F)c1F</smiles>

Fluoro(methyl)(2,3,4,5-tetrafluorophenyl)(thiophen-3-yl)silane (6f). The general procedure was followed with Ru complex $\mathbf{1 b}(2.1 \mathrm{mg}, 3.9 \mu \mathrm{mol}, 1.0 \mathrm{~mol} \%)$ and hydrosilane $\mathbf{5 f}$ 
(117.6 mg, $0.40 \mathrm{mmol}$ ) at $120^{\circ} \mathrm{C}$ for $24 \mathrm{~h}$. The hydrodefluorination product was afforded as a colorless liquid (105.4 mg, 90\% yield). ${ }^{1} \mathrm{H}$ NMR (400 MHz, $\left.\mathrm{CDCl}_{3}\right): \delta 7.77(\mathrm{~s}, 1 \mathrm{H}), 7.52$ $-7.50(\mathrm{~m}, 1 \mathrm{H}), 7.32(\mathrm{~d}, J=4.0 \mathrm{~Hz}, 1 \mathrm{H}), 7.13-7.07(\mathrm{~m}, 1 \mathrm{H}), 0.87(\mathrm{~d}, J=8.0 \mathrm{~Hz}, 3 \mathrm{H}) .{ }^{19} \mathrm{~F}$ $\operatorname{NMR}\left(376 \mathrm{MHz}, \mathrm{CDCl}_{3}\right): \delta-127.3(\mathrm{t}, J=18.8 \mathrm{~Hz}),-137.8--138.0(\mathrm{~m}),-151.0--151.2$ (m), -154.7 (t, $J=22.2 \mathrm{~Hz}),-163.2--163.2(\mathrm{~m}) .{ }^{13} \mathrm{C} \mathrm{NMR}\left(101 \mathrm{MHz}, \mathrm{CDCl}_{3}\right): \delta 150.8$ (dm, C-F, ${ }^{1} J_{C-F}=243.2 \mathrm{~Hz}$ ), 147.7 (ddd, C-F, ${ }^{1} J_{C-F}=251.6 \mathrm{~Hz},{ }^{2} J_{C-F}=9.8 \mathrm{~Hz},{ }^{3} J_{C-F}=3.3$ $\mathrm{Hz}), 142.4\left(\mathrm{dm}, \mathrm{C}-\mathrm{F},{ }^{1} \mathrm{~J}_{\mathrm{C}-\mathrm{F}}=258.4 \mathrm{~Hz}\right), 140.5\left(\mathrm{dm}, \mathrm{C}-\mathrm{F},{ }^{1} \mathrm{~J}_{\mathrm{C}-\mathrm{F}}=259.3 \mathrm{~Hz}\right), 135.8(\mathrm{~d}, \mathrm{C}-\mathrm{F}$, $\left.{ }^{4} J_{C-F}=2.1 \mathrm{~Hz}\right), 133.1\left(\mathrm{~d}, \mathrm{C}-\mathrm{F},{ }^{2} \mathrm{~J}_{\mathrm{C}-\mathrm{F}}=17.7 \mathrm{~Hz}\right), 130.9,127.1,118.1-117.6(\mathrm{~m}), 115.6-$ $115.2(\mathrm{~m}),-1.2\left(\mathrm{~d}, \mathrm{C}-\mathrm{F},{ }^{2} \mathrm{~J}_{\mathrm{C}-\mathrm{F}}=14.4 \mathrm{~Hz}\right.$ ). IR (thin film): 1515, 1451, 1324, 1008, 971, 773. HRMS (El-TOF) calcd. for [ $\left.\mathrm{C}_{11} \mathrm{H}_{7} \mathrm{~F}_{5} \mathrm{SSi}\right]\left([\mathrm{M}]^{+}\right): 293.9958$, found: 293.9955.<smiles>C[Si](c1ccc2sccc2c1)(c1cccs1)c1cc(F)c(F)c(F)c1F</smiles>

Benzo[b]thiophen-5-ylfluoro(methyl)(2,3,4,5-tetrafluorophenyl)silane $(6 \mathrm{~g})$. The general procedure was followed with Ru complex 1b (2.7 mg, $5.0 \mu \mathrm{mol}, 2.5 \mathrm{~mol} \%)$, hydrosilane $5 \mathrm{~g}(68.8 \mathrm{mg}, 0.30 \mathrm{mmol})$, and THF $(0.20 \mathrm{~mL})$ at $120^{\circ} \mathrm{C}$ for $24 \mathrm{~h}$. The hydrodefluorination product was afforded as a colorless liquid (60.2 mg, $88 \%$ yield). ${ }^{1} \mathrm{H} \mathrm{NMR}\left(400 \mathrm{MHz}, \mathrm{CDCl}_{3}\right)$ : $\delta 8.11(\mathrm{~s}, 1 \mathrm{H}), 7.98(\mathrm{~d}, J=8.0 \mathrm{~Hz}, 1 \mathrm{H}), 7.54(\mathrm{~d}, J=8.0 \mathrm{~Hz}, 1 \mathrm{H}), 7.50(\mathrm{~d}, J=4.0 \mathrm{~Hz}, 1 \mathrm{H})$, $7.38(\mathrm{~d}, J=4.0 \mathrm{~Hz}, 1 \mathrm{H}), 7.13-7.07(\mathrm{~m}, 1 \mathrm{H}), 0.91(\mathrm{~d}, J=8.0 \mathrm{~Hz}, 3 \mathrm{H}) .{ }^{19} \mathrm{~F}$ NMR $(376 \mathrm{MHz}$, $\mathrm{CDCl}_{3}$ ): $\delta-126.9$ (t, $\left.J=18.4 \mathrm{~Hz}\right),-137.8--137.9(\mathrm{~m}),-151.0(\mathrm{tt}, J=19.2,7.1 \mathrm{~Hz}),-154.6$ (t, $J=21.1 \mathrm{~Hz}),-165.7$ (q, J=6.8 Hz). ${ }^{13} \mathrm{C} \mathrm{NMR}\left(101 \mathrm{MHz}, \mathrm{CDCl}_{3}\right): \delta 150.6\left(\mathrm{dm}, \mathrm{C}-\mathrm{F},{ }^{1} \mathrm{~J}_{\mathrm{C}}\right.$ $\mathrm{F}=241.0 \mathrm{~Hz}), 147.5\left(\mathrm{ddd}, \mathrm{C}-\mathrm{F},{ }^{1} \mathrm{~J}_{\mathrm{C}-\mathrm{F}}=251.8 \mathrm{~Hz},{ }^{2} \mathrm{~J}_{\mathrm{C}-\mathrm{F}}=10.0 \mathrm{~Hz},{ }^{3} \mathrm{~J}_{\mathrm{C}-\mathrm{F}}=3.3 \mathrm{~Hz}\right), 142.7$, $142.2\left(\mathrm{dm}, \mathrm{C}-\mathrm{F},{ }^{1} \mathrm{~J}_{\mathrm{C}-\mathrm{F}}=258.1 \mathrm{~Hz}\right), 140.3\left(\mathrm{dm}, \mathrm{C}-\mathrm{F},{ }^{1} \mathrm{~J}_{\mathrm{C}-\mathrm{F}}=257.7 \mathrm{~Hz}\right), 139.2,129.4,127.9$, $127.1\left(\mathrm{~d}, \mathrm{C}-\mathrm{F},{ }^{2} \mathrm{~J}_{\mathrm{C}-\mathrm{F}}=16.3 \mathrm{~Hz}\right), 127.0,123.8,122.6,117.9-117.4(\mathrm{~m}), 115.5-115.2(\mathrm{~m})$, $-1.9\left(\mathrm{dd}, \mathrm{C}-\mathrm{F},{ }^{2} J_{\mathrm{C}-\mathrm{F}}=14.1 \mathrm{~Hz},{ }^{4} J_{\mathrm{C}-\mathrm{F}}=1.7 \mathrm{~Hz}\right)$. IR (thin film): $1451,1323,1090,1008,868$, 784. HRMS (El-TOF) calcd. for [ $\left.\mathrm{C}_{15} \mathrm{H}_{9} \mathrm{~F}_{5} \mathrm{SS}\right]$ ([M] $\left.]^{+}\right)$: 344.0114, found: 344.0120. 


\section{Elaboration of the HDF Product 3n}

\subsection{Pd-catalyzed Hiyama-Denmark cross-coupling of $3 \mathrm{n}$ with iodobenzenes}

\section{General procedure}

In an argon-filled glovebox, a $5 \mathrm{~mL}$ dried Schlenk tube was charged with $\mathrm{Pd}\left(\mathrm{PPh}_{3}\right)_{4}$ (12 mg, $10 \mu \mathrm{mol}, 10 \mathrm{~mol} \%), \mathrm{Ag}_{2} \mathrm{O}$ (35 mg, $0.15 \mathrm{mmol}$ ), and THF (1.0 mL). Then, $3 \mathrm{n}(23$ $\mathrm{mg}, 0.10 \mathrm{mmol})$ and Ar-I $(0.10 \mathrm{mmol})$ were added. The tube was sealed tightly with a teflon plug under Ar atmosphere and the mixture was stirred at room temperature for $2 \mathrm{~h}$, and heated at $50{ }^{\circ} \mathrm{C}$ for $24 \mathrm{~h}$. Then the reaction mixture was cooled to room temperature, and filtered through a Celite ${ }^{\circledR}$ plug. The filtrate was collected and the volatiles were removed under reduced pressure. $\mathrm{C}_{6} \mathrm{~F}_{5} \mathrm{H}$ (1.0 equiv) was added as an internal standard and the yield was determined by ${ }^{1} \mathrm{H}$ NMR. The crude mixture was purified by flash column chromatography (silica gel, petroleum ether) to obtain the desired product.

\section{Spectral data for the products of Hiyama-Denmark cross-coupling}<smiles>Fc1cc(-c2ccccc2)c(F)c(F)c1F</smiles>

2,3,4,5-Tetrafluoro-1,1'-biphenyl (4a). The general procedure was followed with $3 \mathrm{n}$ (23 $\mathrm{mg}, 0.10 \mathrm{mmol})$ with iodobenzene $(11 \mu \mathrm{L}, 0.10 \mathrm{mmol})$. The cross-coupling product $4 \mathrm{a}$ was obtained as a white solid ( $21 \mathrm{mg}, 91 \%$ yield). $\mathrm{R}_{f}=0.8$ (petroleum ether). ${ }^{1} \mathrm{H}$ NMR (400 $\left.\mathrm{MHz}, \mathrm{CDCl}_{3}\right): \delta 7.49-7.42(\mathrm{~m}, 5 \mathrm{H}), 7.10-7.03(\mathrm{~m}, 1 \mathrm{H}) .{ }^{19} \mathrm{~F} \mathrm{NMR}\left(376 \mathrm{MHz}, \mathrm{CDCl}_{3}\right): \delta-$ $139.6--139.7(\mathrm{~m}),-143.8--143.8(\mathrm{~m}),-155.2--155.3(\mathrm{~m}),-157.1--157.2(\mathrm{~m}) .{ }^{13} \mathrm{C}$ $\operatorname{NMR}\left(101 \mathrm{MHz}, \mathrm{CDCl}_{3}\right): \delta 147.2\left(\mathrm{ddt}, \mathrm{C}-\mathrm{F},{ }^{1} \mathrm{~J}_{\mathrm{C}-\mathrm{F}}=247.0 \mathrm{~Hz},{ }^{2} \mathrm{~J}_{\mathrm{C}-\mathrm{F}}=9.7 \mathrm{~Hz},{ }^{3} J_{\mathrm{C}-\mathrm{F}}=3.3\right.$ $\mathrm{Hz}$ ), 145.0 (ddd, C-F, $\left.{ }^{1} \mathrm{~J}_{\mathrm{C}-\mathrm{F}}=249.3 \mathrm{~Hz},{ }^{2} \mathrm{~J}_{\mathrm{C}-\mathrm{F}}=10.7 \mathrm{~Hz},{ }^{3} \mathrm{~J}_{\mathrm{C}-\mathrm{F}}=2.4 \mathrm{~Hz}\right), 141.3(\mathrm{dm}, \mathrm{C}-\mathrm{F}$, $\left.{ }^{1} J_{\mathrm{C}-\mathrm{F}}=253.6 \mathrm{~Hz}\right), 139.8\left(\mathrm{dm}, \mathrm{C}-\mathrm{F},{ }^{1} \mathrm{~J}_{\mathrm{C}-\mathrm{F}}=254.4 \mathrm{~Hz}\right), 133.2,129.0,128.9,128.9,125.6-$ $125.4(\mathrm{~m}), 111.5\left(\mathrm{dm}, \mathrm{C}-\mathrm{F},{ }^{2} \mathrm{~J}_{\mathrm{C}-\mathrm{F}}=19.7 \mathrm{~Hz}\right)$. Spectral data is in agreement with published data. $^{4}$ 
<smiles>Cc1ccc(-c2cc(F)c(F)c(F)c2F)cc1</smiles>

2,3,4,5-Tetrafluoro-4'-methyl-1,1'-biphenyl (4b). The general procedure was followed with $3 \mathrm{n}(23 \mathrm{mg}, 0.10 \mathrm{mmol})$ with 4-iodotoluene (22 $\mathrm{mg}, 0.10 \mathrm{mmol})$. The cross-coupling product $\mathbf{4 b}$ was obtained as a colorless liquid (16 mg, $67 \%$ yield). $R_{f}=0.8$ (petroleum ether). ${ }^{1} \mathrm{H}$ NMR (400 MHz, $\mathrm{CDCl}_{3}$ ): $\delta 7.38$ (d, J=7.2 Hz, 2H), 7.28 (d, J=8.0 Hz, 2H), $7.07-7.00$ $(\mathrm{m}, 1 \mathrm{H}), 2.41(\mathrm{~s}, 3 \mathrm{H}) .{ }^{19} \mathrm{~F} \mathrm{NMR}\left(376 \mathrm{MHz}, \mathrm{CDCl}_{3}\right): \delta-139.8--139.9(\mathrm{~m}),-143.9--143.9$ (m), -155.4--155.5 (m), -157.6--157.8 (m). $\left.{ }^{13} \mathrm{C} \mathrm{NMR} \mathrm{(101} \mathrm{MHz,} \mathrm{CDCl}\right)$ ) $\delta 147.2$ (ddt, $\left.\mathrm{C}-\mathrm{F},{ }^{1} \mathrm{~J}_{\mathrm{C}-\mathrm{F}}=247.7,{ }^{2} \mathrm{~J}_{\mathrm{C}-\mathrm{F}}=9.1,{ }^{3} \mathrm{~J}_{\mathrm{C}-\mathrm{F}}=3.8 \mathrm{~Hz}\right), 145.0\left(\mathrm{ddd}, \mathrm{C}-\mathrm{F},{ }^{1} J_{\mathrm{C}-\mathrm{F}}=249.9,{ }^{2} \mathrm{~J}_{\mathrm{C}-\mathrm{F}}=\right.$ 11.6, $\left.{ }^{3} \mathrm{~J}_{\mathrm{C}-\mathrm{F}}=3.3 \mathrm{~Hz}\right), 141.3\left(\mathrm{dm}, \mathrm{C}-\mathrm{F},{ }^{1} \mathrm{~J}_{\mathrm{C}-\mathrm{F}}=253.5 \mathrm{~Hz}\right), 139.6\left(\mathrm{dm}, \mathrm{C}-\mathrm{F},{ }^{1} \mathrm{~J}_{\mathrm{C}-\mathrm{F}}=254.2\right.$ $\mathrm{Hz}), 138.9,129.7,128.8,128.7,125.6-125.4(\mathrm{~m}), 111.3\left(\mathrm{dt}, \mathrm{C}-\mathrm{F},{ }^{2} \mathrm{~J}_{\mathrm{C}-\mathrm{F}}=19.6,{ }^{3} \mathrm{~J}_{\mathrm{C}-\mathrm{F}}=3.1\right.$ $\mathrm{Hz}), 21.4$. Spectral data is in agreement with published data. ${ }^{5}$<smiles>Cc1cccc(-c2cc(F)c(F)c(F)c2F)c1</smiles>

2,3,4,5-Tetrafluoro-3'-methyl-1,1'-biphenyl (4c). The general procedure was followed with $3 \mathrm{n}(23 \mathrm{mg}, 0.10 \mathrm{mmol})$ with 3 -iodotoluene $(13 \mu \mathrm{L}, 0.10 \mathrm{mmol})$. The cross-coupling product $4 \mathrm{c}$ was obtained as a colorless liquid ( $13 \mathrm{mg}, 54 \%$ yield). $R_{f}=0.8$ (petroleum ether). ${ }^{1} \mathrm{H}$ NMR $\left(400 \mathrm{MHz}, \mathrm{CDCl}_{3}\right)$ : $\delta 7.35$ (t, $\left.J=8.0 \mathrm{~Hz}, 1 \mathrm{H}\right), 7.28-7.23(\mathrm{~m}, 3 \mathrm{H}), 7.07-7.02(\mathrm{~m}$, 1H), $2.42(\mathrm{~s}, 3 \mathrm{H}) .{ }^{19} \mathrm{~F} \mathrm{NMR}\left(376 \mathrm{MHz}, \mathrm{CDCl}_{3}\right): \delta-139.8--139.9(\mathrm{~m}),-143.6--143.7(\mathrm{~m})$, $-155.3--155.4(\mathrm{~m}),-157.4--157.4(\mathrm{~m}) .{ }^{13} \mathrm{C}$ NMR (101 MHz, $\left.\mathrm{CDCl}_{3}\right)$ : $\delta 147.1$ (dm, C$\left.\mathrm{F},{ }^{1} \mathrm{~J}_{\mathrm{C}-\mathrm{F}}=248.9 \mathrm{~Hz}\right), 145.0\left(\mathrm{dm}, \mathrm{C}-\mathrm{F},{ }^{1} \mathrm{~J}_{\mathrm{C}-\mathrm{F}}=246.6 \mathrm{~Hz}\right), 141.3\left(\mathrm{dm}, \mathrm{C}-\mathrm{F},{ }^{1} \mathrm{~J}_{\mathrm{C}-\mathrm{F}}=253.4 \mathrm{~Hz}\right)$, $139.7\left(\mathrm{dm}, \mathrm{C}-\mathrm{F},{ }^{1} \mathrm{~J}_{\mathrm{C}-\mathrm{F}}=254.3 \mathrm{~Hz}\right), 138.7,133.2,129.6,129.6,128.8,126.0$ (d, C-F, ${ }^{4} \mathrm{~J}_{\mathrm{C}}$ $\mathrm{F}=2.0 \mathrm{~Hz}), 125.8-125.6(\mathrm{~m}), 111.5\left(\mathrm{dt}, \mathrm{C}-\mathrm{F},{ }^{2} J_{\mathrm{C}-\mathrm{F}}=19.6 \mathrm{~Hz},{ }^{3} J_{\mathrm{C}-\mathrm{F}}=3.1 \mathrm{~Hz}\right), 21.6 . \mathrm{IR}$ (thin film): 2925, 1525, 1482, 1083, 1015, 788, 710. HRMS (El-TOF) calcd. for $\left[\mathrm{C}_{13} \mathrm{H}_{8} \mathrm{~F}_{4}\right]$ $\left([\mathrm{M}]^{+}\right): 240.0562$, found: 240.0564 . 
<smiles>CSc1ccc(-c2cc(F)c(F)c(F)c2F)cc1</smiles>

Methyl(2',3',4',5'-tetrafluoro-[1,1'-biphenyl]-4-yl)sulfane (4d). The general procedure was followed with $3 \mathrm{n}$ (23 $\mathrm{mg}, 0.10 \mathrm{mmol})$ with 4-iodothioanisole (25 mg, $0.10 \mathrm{mmol})$. The cross-coupling product $\mathbf{4 d}$ was obtained as a white solid $\left(21 \mathrm{mg}, 77 \%\right.$ yield). $\mathrm{R}_{f}=0.8$ (petroleum ether). ${ }^{1} \mathrm{H}$ NMR $\left(400 \mathrm{MHz}, \mathrm{CDCl}_{3}\right): \delta 7.40$ (d, $\left.J=8.0 \mathrm{~Hz}, 2 \mathrm{H}\right), 7.32$ (d, $J=8.0 \mathrm{~Hz}$, 2H), $7.07-7.00(\mathrm{~m}, 1 \mathrm{H}), 2.53(\mathrm{~s}, 3 \mathrm{H}) .{ }^{19} \mathrm{~F} \mathrm{NMR}\left(376 \mathrm{MHz}, \mathrm{CDCl}_{3}\right): \delta-139.5--139.6(\mathrm{~m})$, $-143.7--143.8(\mathrm{~m}),-155.1--155.2(\mathrm{~m}),-157.2--157.3(\mathrm{~m}) .{ }^{13} \mathrm{C}$ NMR $(101 \mathrm{MHz}$, $\left.\mathrm{CDCl}_{3}\right): \delta 147.2\left(\mathrm{dm}, \mathrm{C}-\mathrm{F},{ }^{1} \mathrm{~J}_{\mathrm{C}-\mathrm{F}}=247.4 \mathrm{~Hz}\right), 145.0\left(\mathrm{dm}, \mathrm{C}-\mathrm{F},{ }^{1} \mathrm{~J}_{\mathrm{C}-\mathrm{F}}=250.5 \mathrm{~Hz}\right), 141.4$ $\left(\mathrm{dm}, \mathrm{C}-\mathrm{F},{ }^{1} \mathrm{~J}_{\mathrm{C}-\mathrm{F}}=253.5 \mathrm{~Hz}\right), 139.7\left(\mathrm{dm}, \mathrm{C}-\mathrm{F},{ }^{1} \mathrm{~J}_{\mathrm{C}-\mathrm{F}}=255.0 \mathrm{~Hz}\right), 140.1,129.6,129.2(\mathrm{~d}$, $\left.\mathrm{C}-\mathrm{F},{ }^{3} \mathrm{~J}_{\mathrm{C}-\mathrm{F}}=3.0 \mathrm{~Hz}\right), 126.5,125.1-124.8(\mathrm{~m}), 111.2\left(\mathrm{dt}, \mathrm{C}-\mathrm{F},{ }^{2} \mathrm{~J}_{\mathrm{C}-\mathrm{F}}=20.2 \mathrm{~Hz},{ }^{3} \mathrm{~J}_{\mathrm{C}-\mathrm{F}}=3.0\right.$ $\mathrm{Hz}$ ), 15.6. IR (thin film): 2922, 1470, 1070, 989, 846, 814, 721. HRMS (EI-TOF) calcd. for $\left[\mathrm{C}_{13} \mathrm{H}_{8} \mathrm{~F}_{4} \mathrm{~S}\right]\left([\mathrm{M}]^{+}\right): 272.0283$, found: 272.0284 .<smiles>Fc1cc(-c2ccc(Cl)cc2)c(F)c(F)c1F</smiles>

4'-Chloro-2,3,4,5-tetrafluoro-1,1'-biphenyl (4e). The general procedure was followed with $3 \mathrm{n}(23 \mathrm{mg}, 0.10 \mathrm{mmol})$ with 1-chloro-4-iodobenzene $(26 \mathrm{mg}, 0.10 \mathrm{mmol})$. The crosscoupling product $4 \mathrm{e}$ was obtained as a white solid (17 mg, $65 \%$ yield). $R_{f}=0.8$ (petroleum ether). ${ }^{1} \mathrm{H}$ NMR $\left(400 \mathrm{MHz}, \mathrm{CDCl}_{3}\right): \delta 7.49-7.40(\mathrm{~m}, 4 \mathrm{H}), 7.06-7.00(\mathrm{~m}, 1 \mathrm{H}) .{ }^{19} \mathrm{~F}$ NMR $\left(376 \mathrm{MHz}, \mathrm{CDCl}_{3}\right): \delta-139.1--139.2(\mathrm{~m}),-143.5--143.5(\mathrm{~m}),-154.7--154.8(\mathrm{~m}),-$ $156.2--156.4(\mathrm{~m}) .{ }^{13} \mathrm{C}$ NMR $\left(101 \mathrm{MHz}, \mathrm{CDCl}_{3}\right): \delta 147.3\left(\mathrm{dm}, \mathrm{C}-\mathrm{F},{ }^{1} \mathrm{~J}_{\mathrm{C}-\mathrm{F}}=233.3 \mathrm{~Hz}\right)$, $144.9\left(\mathrm{dm}, \mathrm{C}-\mathrm{F},{ }^{1} \mathrm{~J}_{\mathrm{C}-\mathrm{F}}=230.3 \mathrm{~Hz}\right), 141.4\left(\mathrm{dm}, \mathrm{C}-\mathrm{F},{ }^{1} \mathrm{~J}_{\mathrm{C}-\mathrm{F}}=253.5 \mathrm{~Hz}\right), 139.5$ (dm, C-F, $\left.{ }^{1} J_{C-F}=255.5 \mathrm{~Hz}\right), 135.2,131.6,130.2\left(\mathrm{~d}, \mathrm{C}-\mathrm{F},{ }^{3} J_{\mathrm{C}-\mathrm{F}}=3.0 \mathrm{~Hz}\right), 129.3,124.4-124.2(\mathrm{~m})$, 111.3 (dt, C-F, ${ }^{2} J_{C-F}=20.2 \mathrm{~Hz},{ }^{3} J_{C-F}=3.0 \mathrm{~Hz}$ ). IR (thin film): 1527, 1482, 1082, 994, 822. HRMS (El-TOF) calcd. for $\left[\mathrm{C}_{12} \mathrm{H}_{5} \mathrm{CIF}_{4}\right]\left([\mathrm{M}]^{+}\right): 260.0016$, found: 260.0011 . 
<smiles>O=[N+]([O-])c1ccc(-c2cc(F)c(F)c(F)c2F)cc1</smiles>

2,3,4,5-Tetrafluoro-4'-nitro-1,1'-biphenyl (4f). The general procedure was followed with 3n (23 mg, $0.10 \mathrm{mmol})$ with 4-iodonitrobenzene (25 mg, $0.10 \mathrm{mmol})$. The cross-coupling product $4 \mathrm{f}$ was obtained as a white solid ( $23 \mathrm{mg}, 85 \%$ yield). $R_{f}=0.8$ (petroleum ether / EtOAc $=100: 1) .{ }^{1} \mathrm{H}$ NMR $\left(400 \mathrm{MHz}, \mathrm{CDCl}_{3}\right): \delta 8.33(\mathrm{~d}, J=8.0 \mathrm{~Hz}, 2 \mathrm{H}), 7.67(\mathrm{~d}, J=8.0$ $\mathrm{Hz}, 2 \mathrm{H}), 7.14-7.08(\mathrm{~m}, 1 \mathrm{H}) .{ }^{19} \mathrm{~F}$ NMR $\left(376 \mathrm{MHz}, \mathrm{CDCl}_{3}\right): \delta-138.0--138.2(\mathrm{~m}),-142.5$ $--142.6(\mathrm{~m}),-153.6--153.98(\mathrm{~m}),-153.9--154.0(\mathrm{~m}) .{ }^{13} \mathrm{C} \mathrm{NMR}\left(101 \mathrm{MHz}, \mathrm{CDCl}_{3}\right): \delta$ 147.9, $147.3\left(\mathrm{dm}, \mathrm{C}-\mathrm{F},{ }^{1} \mathrm{~J}_{\mathrm{C}-\mathrm{F}}=249.9 \mathrm{~Hz}\right), 145.0\left(\mathrm{dm}, \mathrm{C}-\mathrm{F},{ }^{1} \mathrm{~J}_{\mathrm{C}-\mathrm{F}}=250.0 \mathrm{~Hz}\right), 141.4(\mathrm{dm}$, $\left.\mathrm{C}-\mathrm{F},{ }^{1} \mathrm{~J}_{\mathrm{C}-\mathrm{F}}=256.9 \mathrm{~Hz}\right), 140.7\left(\mathrm{dm}, \mathrm{C}-\mathrm{F},{ }^{1} \mathrm{~J}_{\mathrm{C}-\mathrm{F}}=258.3 \mathrm{~Hz}\right), 139.3,129.8\left(\mathrm{~d}, \mathrm{C}-\mathrm{F},{ }^{3} \mathrm{~J}_{\mathrm{C}-\mathrm{F}}=\right.$ 3.0 Hz), 124.0, $123.1-122.9(\mathrm{~m}), 111.4\left(\mathrm{dt}, \mathrm{C}-\mathrm{F},{ }^{2} \mathrm{~J}_{\mathrm{C}-\mathrm{F}}=19.2 \mathrm{~Hz},{ }^{3} \mathrm{~J}_{\mathrm{C}-\mathrm{F}}=3.0 \mathrm{~Hz}\right)$. IR (thin film): 2922, 1521, 1474, 1345, 1079, 1000, 839, 706. HRMS (El-TOF) calcd. for $\left[\mathrm{C}_{12} \mathrm{H}_{5} \mathrm{~F}_{4} \mathrm{NO}_{2}\right]\left([\mathrm{M}]^{+}\right): 271.0256$, found: 271.0256 .<smiles>COc1cc(OC)cc(-c2cc(F)c(F)c(F)c2F)c1</smiles>

2,3,4,5-Tetrafluoro-3',5'-dimethoxy-1,1'-biphenyl (4g). The general procedure was followed with $3 \mathrm{n}$ (23 mg, $0.10 \mathrm{mmol})$ with 1-iodo-3,5-dimethoxybenzene (26 mg, $0.10 \mathrm{mmol})$. The cross-coupling product $\mathbf{4 g}$ was obtained as a white solid ( $23 \mathrm{mg}, 79 \%$ yield). $\mathrm{R}_{f}=0.8$ (petroleum ether / EtOAc = $100: 1) .{ }^{1} \mathrm{H}$ NMR $\left(400 \mathrm{MHz}, \mathrm{CDCl}_{3}\right): \delta 7.09-7.03(\mathrm{~m}, 1 \mathrm{H})$, $6.60(\mathrm{~s}, 2 \mathrm{H}), 6.52(\mathrm{~s}, 1 \mathrm{H}), 3.83(\mathrm{~s}, 6 \mathrm{H}) .{ }^{19} \mathrm{~F} \mathrm{NMR}\left(376 \mathrm{MHz}, \mathrm{CDCl}_{3}\right): \delta-139.6--139.7(\mathrm{~m})$, $-143.0--143.0(\mathrm{~m}),-155.1--155.2(\mathrm{~m}),-156.9--157.0(\mathrm{~m}) .{ }^{13} \mathrm{C}$ NMR $(101 \mathrm{MHz}$, $\left.\mathrm{CDCl}_{3}\right): \delta 161.1,147.1\left(\mathrm{dm}, \mathrm{C}-\mathrm{F},{ }^{1} \mathrm{~J}_{\mathrm{C}-\mathrm{F}}=258.6 \mathrm{~Hz}\right), 145.0\left(\mathrm{dm}, \mathrm{C}-\mathrm{F},{ }^{1} \mathrm{~J}_{\mathrm{C}-\mathrm{F}}=249.5 \mathrm{~Hz}\right)$, $141.3\left(\mathrm{dm}, \mathrm{C}-\mathrm{F},{ }^{1} \mathrm{~J}_{\mathrm{C}-\mathrm{F}}=253.5 \mathrm{~Hz}\right), 139.9\left(\mathrm{dm}, \mathrm{C}-\mathrm{F},{ }^{1} \mathrm{~J}_{\mathrm{C}-\mathrm{F}}=254.5 \mathrm{~Hz}\right), 135.0,125.6-125.4$ (m), $111.5\left(\mathrm{dt}, \mathrm{C}-\mathrm{F},{ }^{2} \mathrm{~J}_{\mathrm{C}-\mathrm{F}}=19.2 \mathrm{~Hz},{ }^{3} \mathrm{~J}_{\mathrm{C}-\mathrm{F}}=3.0 \mathrm{~Hz}\right), 107.2\left(\mathrm{~d}, \mathrm{C}-\mathrm{F},{ }^{3} \mathrm{~J}_{\mathrm{C}-\mathrm{F}}=3.0 \mathrm{~Hz}\right), 100.8$, 55.6. IR (thin film): 2924, 1591, 1451, 1201, 1152, 1058, 922, 831, 687. HRMS (El-TOF) calcd. for $\left[\mathrm{C}_{14} \mathrm{H}_{10} \mathrm{~F}_{4} \mathrm{O}_{2}\right]\left([\mathrm{M}]^{+}\right): 286.0617$, found: 286.0622 . 
<smiles>Fc1cc(-c2cccc3ccccc23)c(F)c(F)c1F</smiles>

1-(2,3,4,5-Tetrafluorophenyl)naphthalene $(4 \mathrm{~h})$. The general procedure was followed with $3 \mathrm{n}(23 \mathrm{mg}, 0.10 \mathrm{mmol})$ with 1-iodonaphthalene (15 $\mu \mathrm{L}, 0.10 \mathrm{mmol})$. The cross-coupling product $4 \mathrm{~h}$ was obtained as a white solid ( $19 \mathrm{mg}, 67 \%$ yield). $\mathrm{R}_{f}=0.8$ (petroleum ether). ${ }^{1} \mathrm{H}$ NMR (400 MHz, $\left.\mathrm{CDCl}_{3}\right): \delta 7.95$ (t, $\left.J=8.0 \mathrm{~Hz}, 2 \mathrm{H}\right), 7.61-7.48(\mathrm{~m}, 4 \mathrm{H}), 7.41$ (d, $J=8.0 \mathrm{~Hz}, 1 \mathrm{H}), 7.09-7.02(\mathrm{~m}, 1 \mathrm{H}) .{ }^{19} \mathrm{~F}$ NMR $\left(376 \mathrm{MHz}, \mathrm{CDCl}_{3}\right)$ : $\delta-138.9--139.0(\mathrm{~m})$, $-139.6--139.7(\mathrm{~m}),-155.1--155.2(\mathrm{~m}),-156.3--156.4(\mathrm{~m}) .{ }^{13} \mathrm{C}$ NMR $(101 \mathrm{MHz}$, $\left.\mathrm{CDCl}_{3}\right): \delta 146.8\left(\mathrm{dm}, \mathrm{C}-\mathrm{F},{ }^{1} \mathrm{~J}_{\mathrm{C}-\mathrm{F}}=249.2 \mathrm{~Hz}\right), 145.2\left(\mathrm{dm}, \mathrm{C}-\mathrm{F},{ }^{1} \mathrm{~J}_{\mathrm{C}-\mathrm{F}}=248.2 \mathrm{~Hz}\right), 140.9$ $\left(\mathrm{dm}, \mathrm{C}-\mathrm{F},{ }^{1} \mathrm{~J}_{\mathrm{C}-\mathrm{F}}=254.6 \mathrm{~Hz}\right), 140.1\left(\mathrm{dm}, \mathrm{C}-\mathrm{F},{ }^{1} \mathrm{~J}_{\mathrm{C}-\mathrm{F}}=254.5 \mathrm{~Hz}\right), 133.6,131.3,130.7,129.4$, $128.5,127.9,126.8,126.3,125.2,124.9,124.3-124.1(\mathrm{~m}), 113.2\left(\mathrm{dt}, \mathrm{C}-\mathrm{F},{ }^{2} J_{\mathrm{C}-\mathrm{F}}=19.2\right.$ $\mathrm{Hz},{ }^{3} \mathrm{~J}_{\mathrm{C}-\mathrm{F}}=3.0 \mathrm{~Hz}$ ). IR (thin film): 3059, 1521, 1476, 1405, 976, 872, 775. HRMS (El-TOF) calcd. for $\left[\mathrm{C}_{16} \mathrm{H}_{8} \mathrm{~F}_{4}\right]\left([\mathrm{M}]^{+}\right)$: 276.0562, found: 276.0556 .<smiles>Fc1cc(-c2cccs2)c(F)c(F)c1F</smiles>

2-(2,3,4,5-Tetrafluorophenyl)thiophene (4i). The general procedure was followed with 3n (23 mg, $0.10 \mathrm{mmol})$ with 2-iodothiophene $(11 \mu \mathrm{L}, 0.10 \mathrm{mmol})$. The cross-coupling product $4 \mathbf{i}$ was obtained as a white solid $\left(12 \mathrm{mg}, 52 \%\right.$ yield). $\mathrm{R}_{f}=0.8$ (petroleum ether). ${ }^{1} \mathrm{H}$ $\operatorname{NMR}\left(400 \mathrm{MHz}, \mathrm{CDCl}_{3}\right): \delta 7.44(\mathrm{~d}, J=4.0 \mathrm{~Hz}, 2 \mathrm{H}), 7.24-7.19(\mathrm{~m}, 1 \mathrm{H}), 7.14(\mathrm{t}, J=4.0 \mathrm{~Hz}$, 1H). ${ }^{19} \mathrm{~F}$ NMR (376 MHz, $\left.\mathrm{CDCl}_{3}\right): \delta-139.2--139.3(\mathrm{~m}),-140.2--140.2(\mathrm{~m}),-154.9--$ $155.0(\mathrm{~m}),-157.1--157.2(\mathrm{~m}) .{ }^{13} \mathrm{C} \mathrm{NMR}\left(101 \mathrm{MHz}, \mathrm{CDCl}_{3}\right): \delta 147.1\left(\mathrm{dm}, \mathrm{C}-\mathrm{F},{ }^{1} \mathrm{~J}_{\mathrm{C}-\mathrm{F}}=\right.$ 247.2 Hz), $144.4\left(\mathrm{dm}, \mathrm{C}-\mathrm{F},{ }^{1} \mathrm{~J}_{\mathrm{C}-\mathrm{F}}=251.5 \mathrm{~Hz}\right), 141.4\left(\mathrm{dm}, \mathrm{C}-\mathrm{F},{ }^{1} \mathrm{~J}_{\mathrm{C}-\mathrm{F}}=253.7 \mathrm{~Hz}\right), 139.4$ $\left(\mathrm{dm}, \mathrm{C}-\mathrm{F},{ }^{1} \mathrm{~J}_{\mathrm{C}-\mathrm{F}}=255.7 \mathrm{~Hz}\right), 134.1,128.0,127.4\left(\mathrm{~d}, \mathrm{C}-\mathrm{F},{ }^{3} \mathrm{~J}_{\mathrm{C}-\mathrm{F}}=6.2 \mathrm{~Hz}\right), 127.0(\mathrm{~d}, \mathrm{C}-\mathrm{F}$, $\left.{ }^{3} J_{C-F}=3.7 \mathrm{~Hz}\right), 119.0-118.7(\mathrm{~m}), 109.5\left(\mathrm{dt}, \mathrm{C}-\mathrm{F},{ }^{2} \mathrm{~J}_{\mathrm{C}-\mathrm{F}}=20.6 \mathrm{~Hz},{ }^{3} \mathrm{~J}_{\mathrm{C}-\mathrm{F}}=3.2 \mathrm{~Hz}\right) . \mathrm{IR}$ (thin film): 2923, 1611, 1474, 1075, 975, 829, 703. HRMS (El-TOF) calcd. for $\left[\mathrm{C}_{10} \mathrm{H}_{4} \mathrm{~F}_{4} \mathrm{~S}\right]\left([\mathrm{M}]^{+}\right)$: 231.9970, found: 231.9961. 


\subsection{TBAF-promoted desilylprotonation of $3 n$}

In an argon-filled glovebox, a $5 \mathrm{~mL}$ dried Schlenk tube was charged with $3 \mathrm{n}$ (34 mg, $0.15 \mathrm{mmol})$ and $\mathrm{CH}_{3} \mathrm{CN}(1.5 \mathrm{~mL})$. Then, TBAF $(0.45 \mathrm{~mL}, 0.45 \mathrm{mmol})$ was added. The tube was sealed tightly with a teflon plug under Ar atmosphere and the mixture was stirred at room temperature for $2 \mathrm{~h}$. After that, $\mathrm{C}_{6} \mathrm{H}_{5} \mathrm{~F}$ ( 0.50 equiv) was added as an internal standard and a quantitative yield of 1,2,3,4-tetrafluorobenzene was determined by ${ }^{19} \mathrm{~F}$ NMR. ${ }^{19} \mathrm{~F}$ NMR $\left(376 \mathrm{MHz}, \mathrm{CDCl}_{3}\right): \delta-140.3,-157.4$. The ${ }^{19} \mathrm{~F}$ NMR was consistent with the spectra of 1,2,3,4-trifluorobenzene reported in the literature. ${ }^{6}$ 


\section{References}

(1) Zhang, Y.; Fang, H.; Yao, W.; Leng, X.; Huang, Z., Synthesis of Pincer Hydrido Ruthenium Olefin Complexes for Catalytic Alkane Dehydrogenation. Organometallics 2016, 35, 181-188.

(2) Moulton, C. J.; Shaw, B. L., Transition Metal-Carbon Bonds .42. Complexes of Nickel, Palladium, Platinum, Rhodium and Iridium with Tridentate Ligand 2,6-Bis (di-tertbutylphosphino)methyl Phenyl. J. Chem. Soc., Dalton Trans. 1976, 1020-1024.

(3) Kumar, A.; Zhou, T.; Emge, T. J.; Mironov, O.; Saxton, R. J.; Krogh-Jespersen, K.; Goldman, A. S., Dehydrogenation of $n$-Alkanes by Solid-Phase Molecular Pincer-Iridium Catalysts. High Yields of alpha-Olefin Product. J. Am. Chem. Soc. 2015, 137, 9894-9911.

(4) Wei, Y.; Kan, J.; Wang, M.; Su, W.; Hong, M., Palladium-Catalyzed Direct Arylation of Electron-Deficient Polyfluoroarenes with Arylboronic Acids. Org. Lett. 2009, 11, 3346-3349.

(5) Mao, S.; Shi, X.; Soulé, J.-F.; Doucet, H., Pd/C as Heterogeneous Catalyst for the Direct Arylation of (Poly)fluorobenzenes. Chem. - Eur. J. 2019, 25, 9504-9513.

(6) Allen, K. J.; Bolton, R.; Williams, G. H., Homolytic Reactions of Polyfluoroaromatic Compounds. Part 16. Competitive Phenylation of Polyfluorobenzenes. J. Chem. Soc., Perkin Trans. 2 1983, 691-695.

(7) Kikushima, K.; Grellier, M.; Ohashi, M.; Ogoshi, S., Transition-Metal-Free Catalytic Hydrodefluorination of Polyfluoroarenes by Concerted Nucleophilic Aromatic Substitution with a Hydrosilicate. Angew. Chem. Int. Ed. 2017, 56, 16191-16196. 


\section{NMR Spectra}

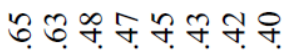

isirivis

ำ은 은

- $\infty \circ \infty$

nininis

이어웅

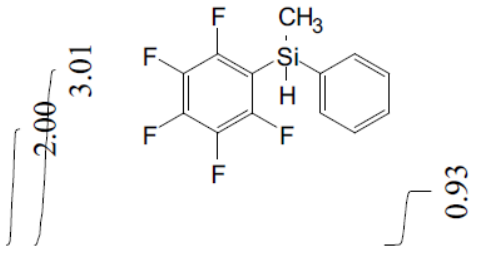

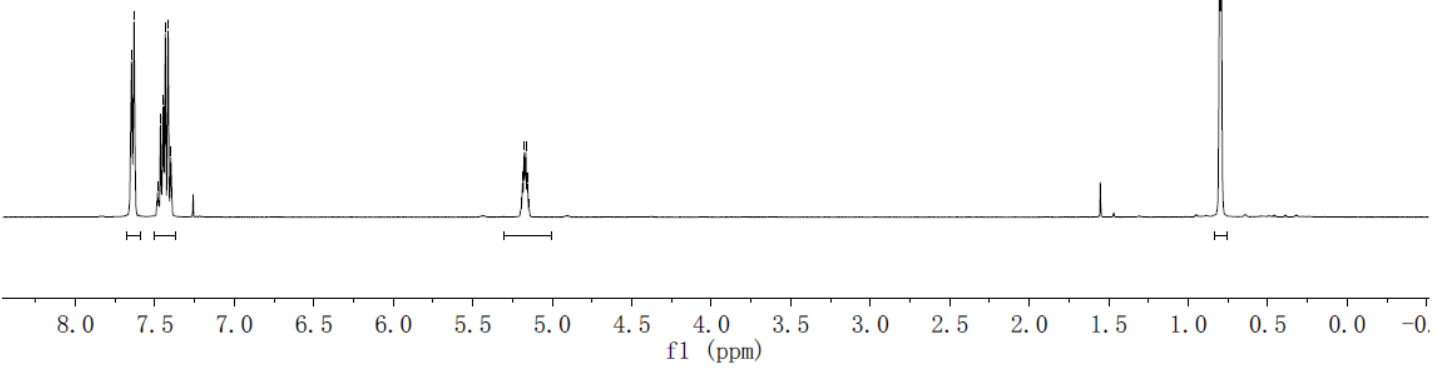

${ }^{1} \mathrm{H}$ NMR (400 MHz, $\mathrm{CDCl}_{3}$ ) of $\mathbf{2 a}$

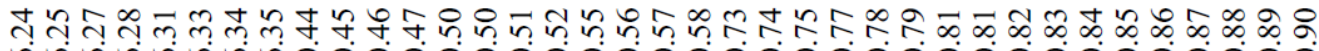
눤원원워<smiles>C[Si](c1ccccc1)c1c(F)c(F)c(F)c(F)c1F</smiles>

ปุ

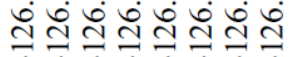

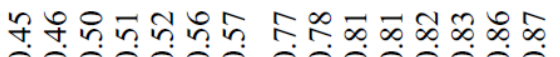

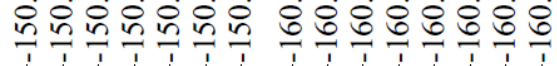

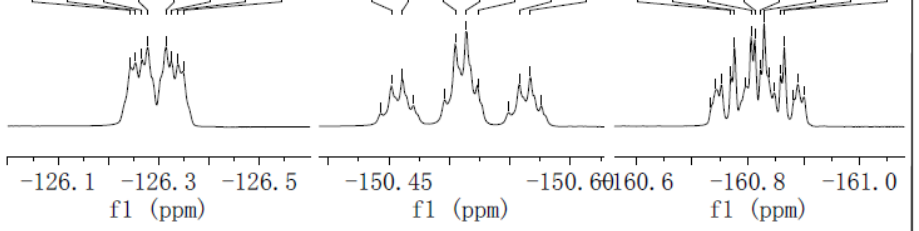

f1 (ppm)

f1 (ppm)

f1 (ppm)

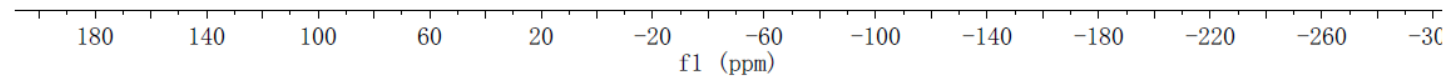

$\left.{ }^{19} \mathrm{~F} \mathrm{NMR}\left(376 \mathrm{MHz}^{\mathrm{CDCl}}\right)_{3}\right)$ of $\mathbf{2 a}$ 
焦<smiles>C[Si](c1ccccc1)c1c(F)c(F)c(F)c(F)c1F</smiles>

\section{우윰ำ

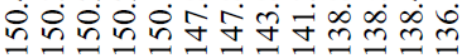

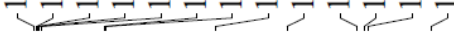

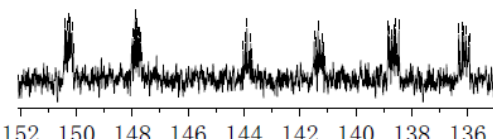

$\begin{array}{lllllllll}152 & 150 & 148 & 146 & 144 & 142 & 140 & 138 & 136\end{array}$

$\mathrm{f} 1(\mathrm{ppm})$

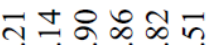

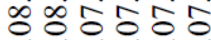

$1=0$
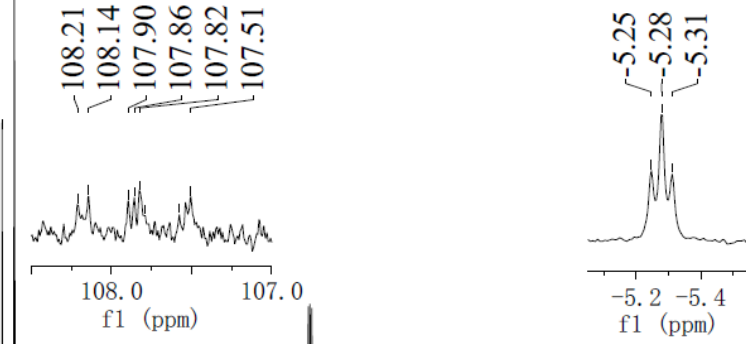

f1 (ppm)

$\begin{array}{lllllllllllllllllllllllllll}230 & 220 & 210 & 200 & 190 & 180 & 170 & 160 & 150 & 140 & 130 & 120 & 110 & 100 & 90 & 80 & 70 & 60 & 50 & 40 & 30 & 20 & 10 & 0 & -10\end{array}$

${ }^{13} \mathrm{C} \mathrm{NMR}\left(101 \mathrm{MHz}, \mathrm{CDCl}_{3}\right)$ of $\mathbf{2 a}$ 


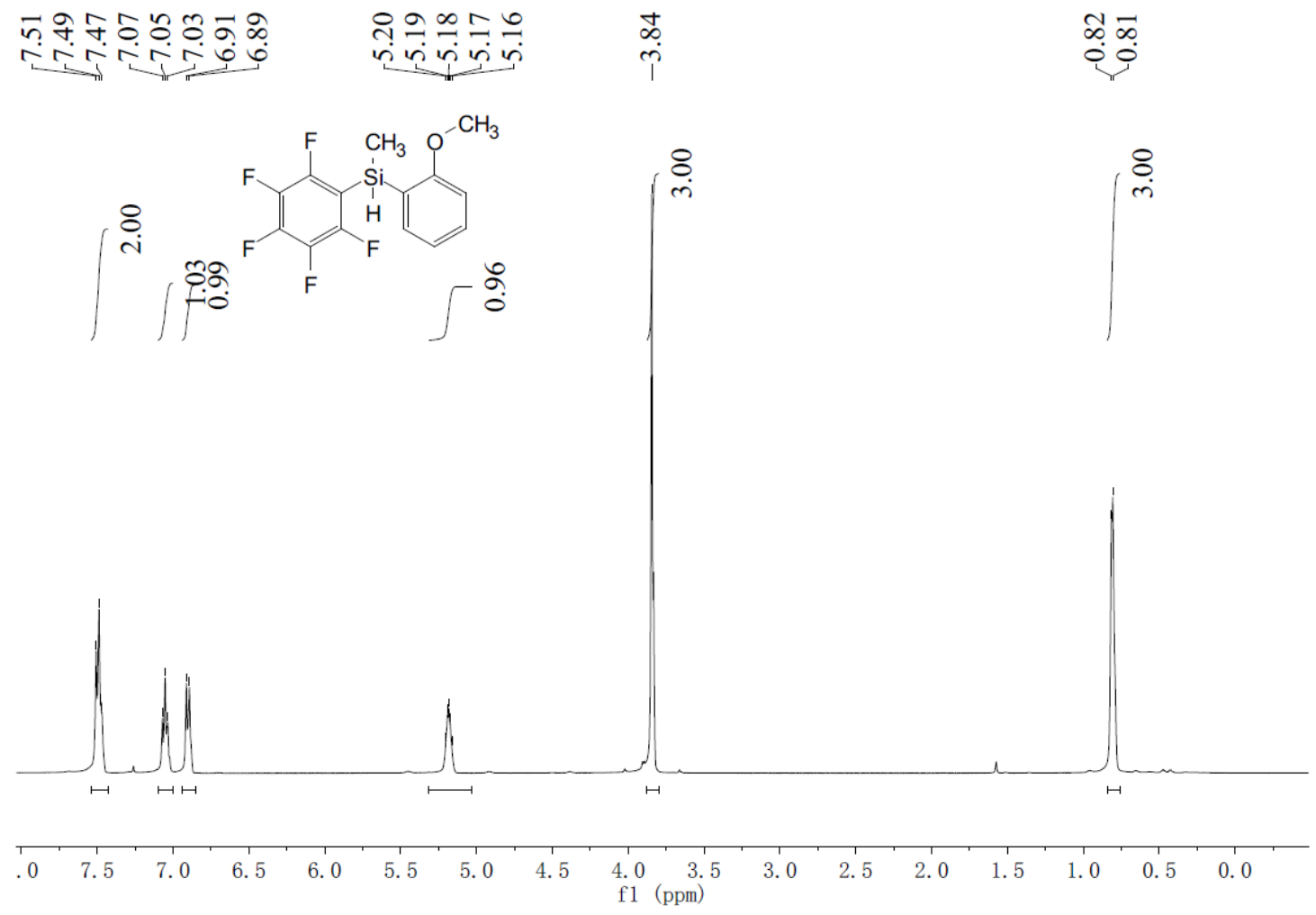

${ }^{1} \mathrm{H} \mathrm{NMR}\left(400 \mathrm{MHz}, \mathrm{CDCl}_{3}\right)$ of $\mathbf{2} \mathbf{b}$
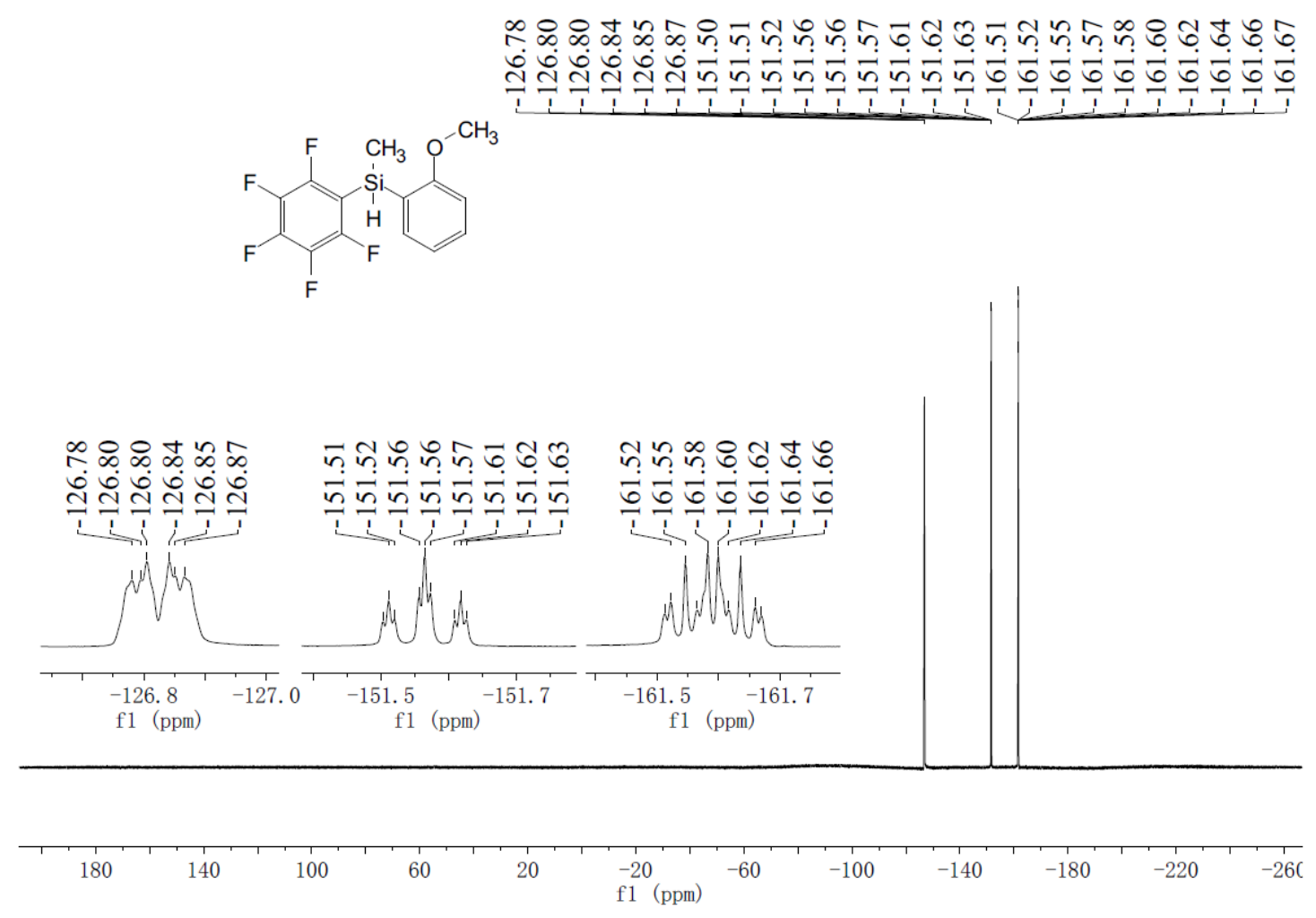

${ }^{19} \mathrm{~F} \mathrm{NMR} \mathrm{(376} \mathrm{MHz,} \mathrm{CDCl}_{3}$ ) of $\mathbf{2 b}$ 


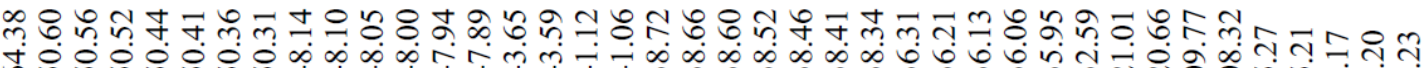

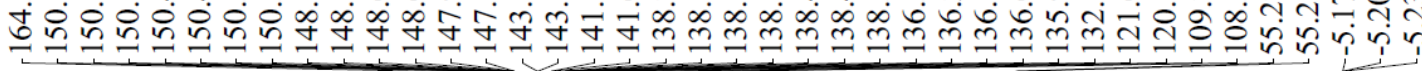<smiles>COc1ccccc1[Si](C)(C)c1c(F)c(F)c(F)c(F)c1F</smiles>

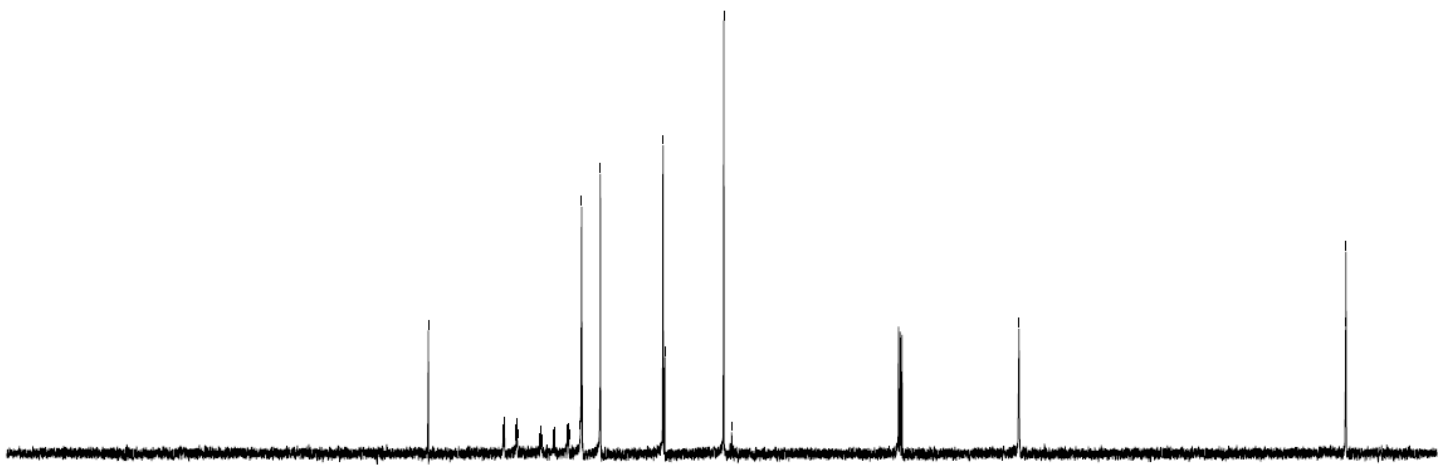

$240 \begin{array}{llllllllllllllllllllllllll}230 & 220 & 210 & 200 & 190 & 180 & 170 & 160 & 150 & 140 & 130 & 120 & 110 & 100 & 90 & 80 & 70 & 60 & 50 & 40 & 30 & 20 & 10 & 0 & -10 & -2\end{array}$ f1 (ppm)

${ }^{13} \mathrm{C}$ NMR $\left(101 \mathrm{MHz}, \mathrm{CDCl}_{3}\right)$ of $\mathbf{2 b}$ 


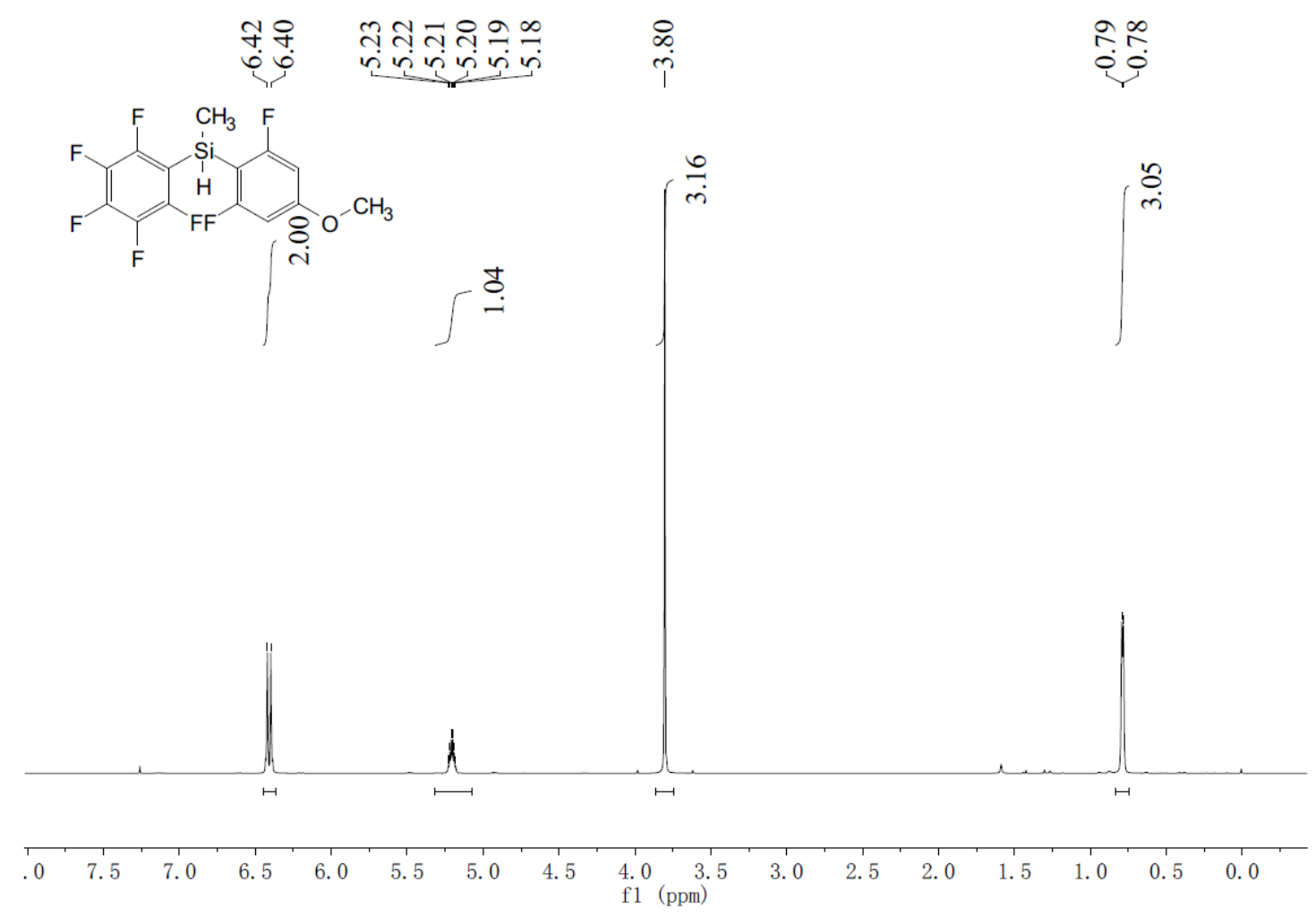

${ }^{1} \mathrm{H} \mathrm{NMR}\left(400 \mathrm{MHz}, \mathrm{CDCl}_{3}\right)$ of $2 \mathrm{c}$
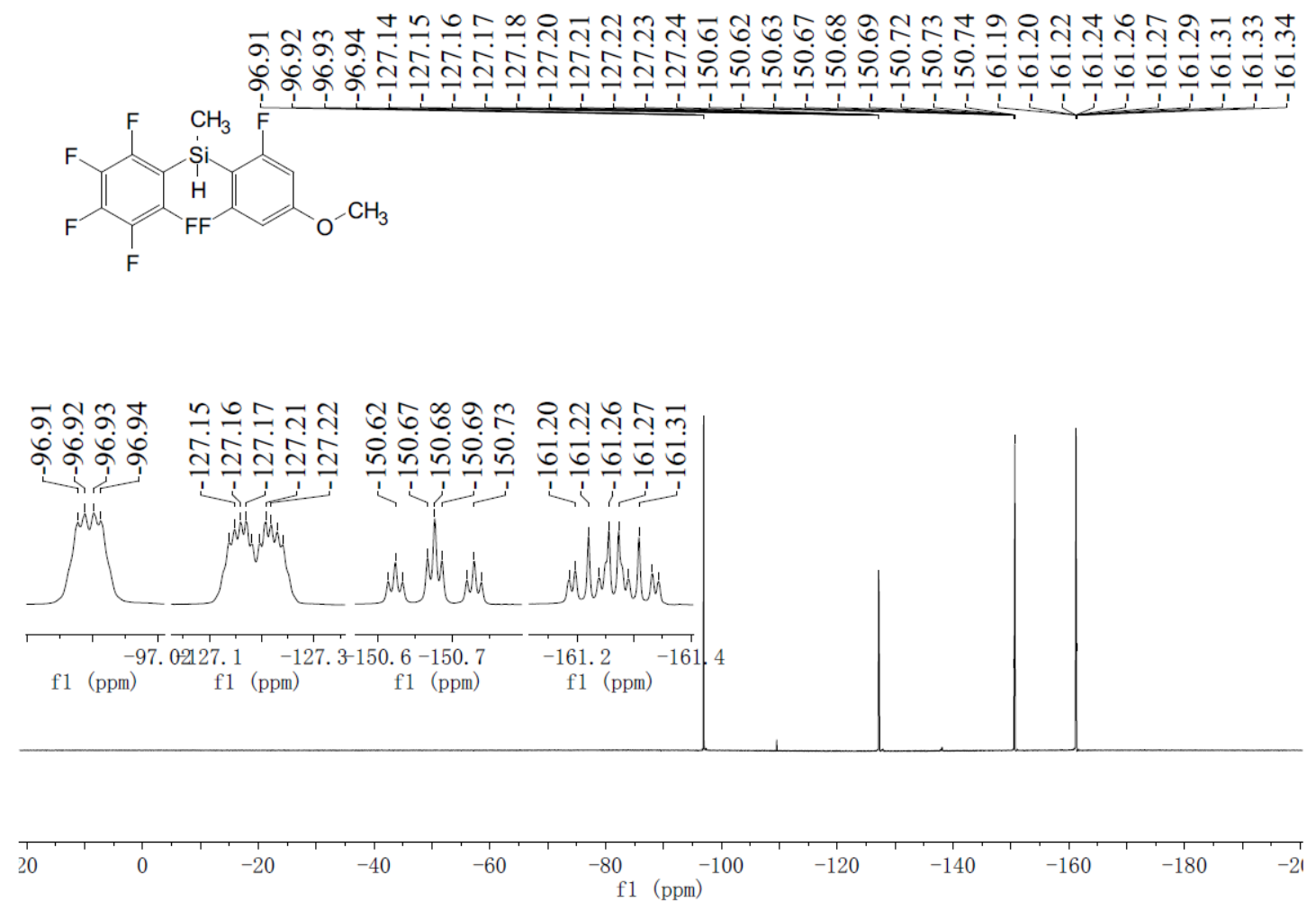

$\left.{ }^{19} \mathrm{~F} \mathrm{NMR} \mathrm{(376} \mathrm{MHz,} \mathrm{CDCl}_{3}\right)$ of $\mathbf{2 c}$ 


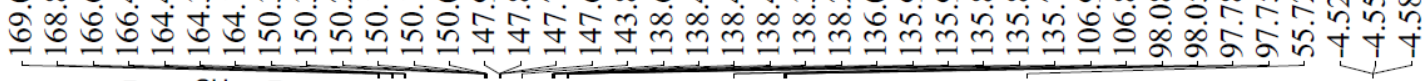<smiles></smiles>

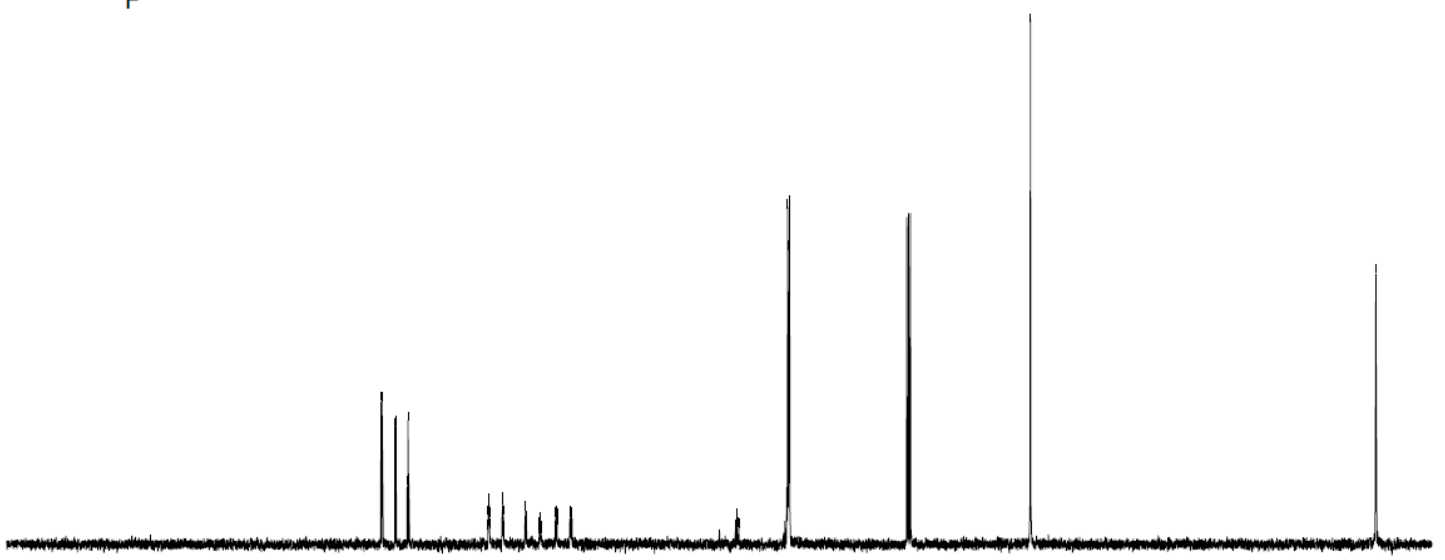

$\begin{array}{lllllllllllllllllllllllllllll}230 & 220 & 210 & 200 & 190 & 180 & 170 & 160 & 150 & 140 & 130 & 120 & 110 & 100 & 90 & 80 & 70 & 60 & 50 & 40 & 30 & 20 & 10 & 0 & -10\end{array}$ f1 (ppm)

$\left.{ }^{13} \mathrm{C} \mathrm{NMR} \mathrm{(101} \mathrm{MHz,} \mathrm{CDCl}_{3}\right)$ of $2 \mathrm{c}$ 

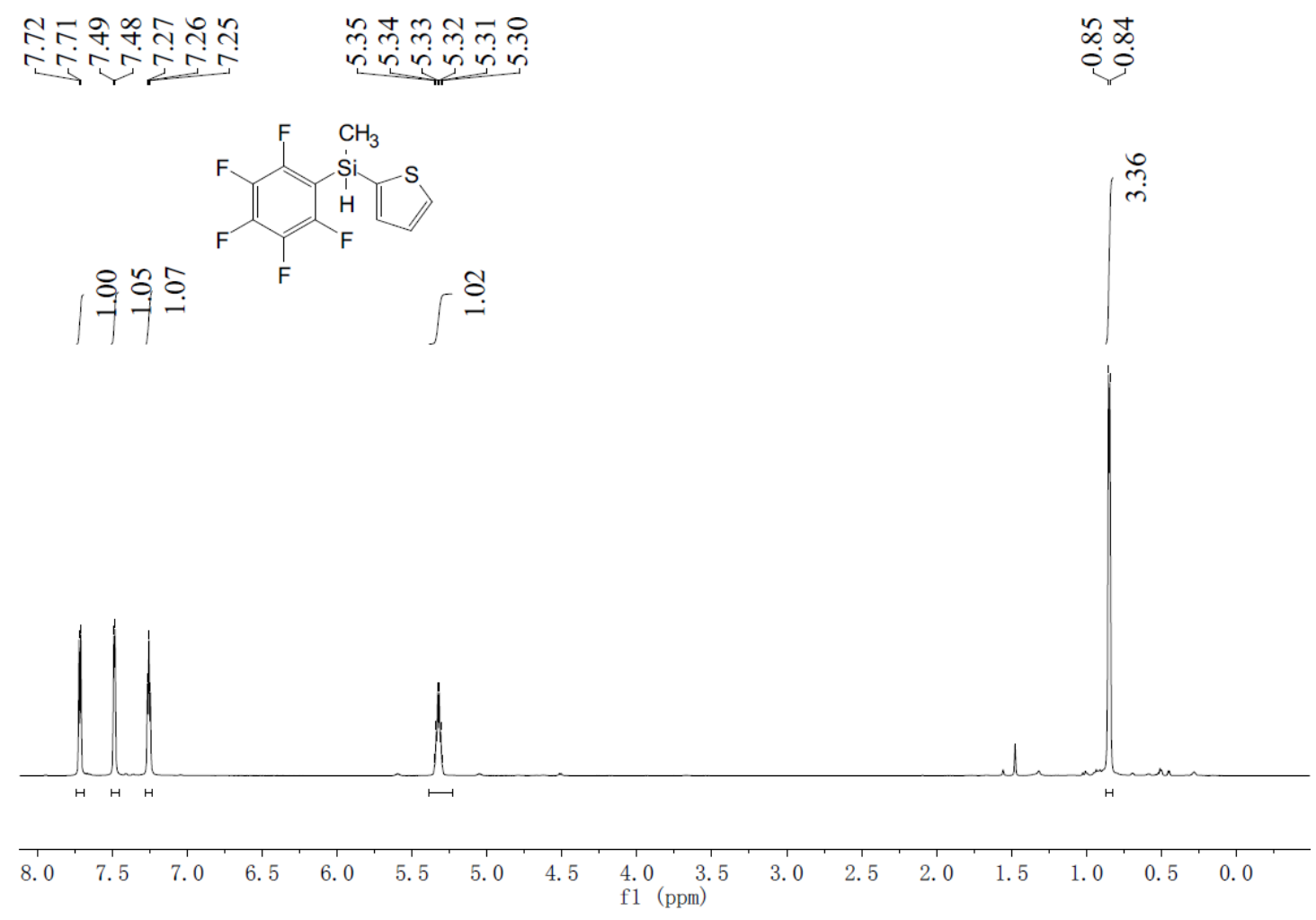

${ }^{1} \mathrm{H}$ NMR $\left(400 \mathrm{MHz}, \mathrm{CDCl}_{3}\right)$ of $\mathbf{2 d}$

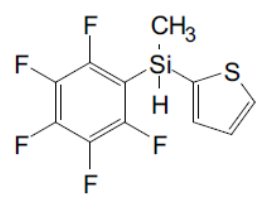

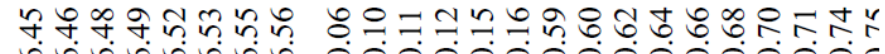

군

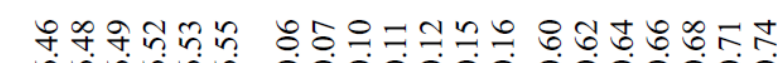

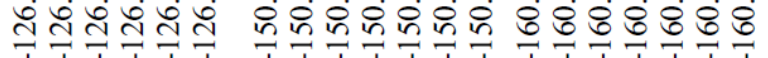

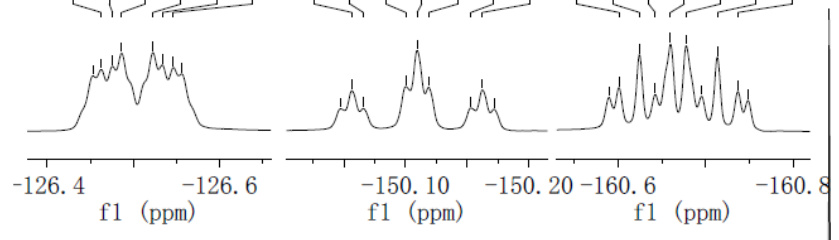

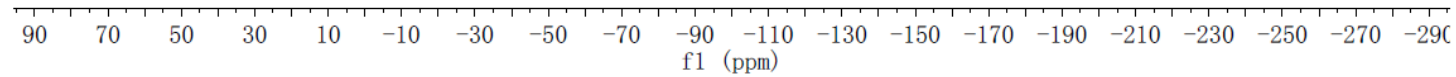

$\left.{ }^{19} \mathrm{~F} \mathrm{NMR} \mathrm{(376} \mathrm{MHz,} \mathrm{CDCl}_{3}\right)$ of $\mathbf{2 d}$ 


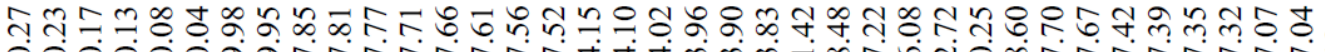

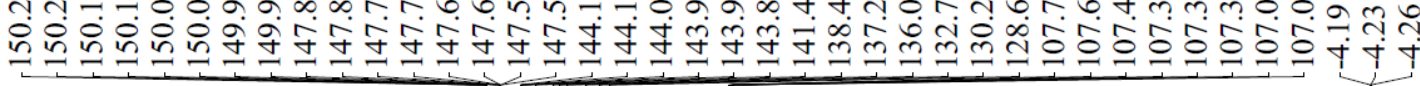<smiles>Cc1c(F)c(F)c(F)c(F)c1C1CC2CCC(C2)C1</smiles>

$\begin{array}{rlllllllllllllllllllllllll}230 & 220 & 210 & 200 & 190 & 180 & 170 & 160 & 150 & 140 & 130 & 120 & 110 & 100 & 90 & 80 & 70 & 60 & 50 & 40 & 30 & 20 & 10 & 0 & -10\end{array}$

$\left.{ }^{13} \mathrm{C} \mathrm{NMR} \mathrm{(101} \mathrm{MHz,} \mathrm{CDCl}_{3}\right)$ of $\mathbf{2 d}$ 


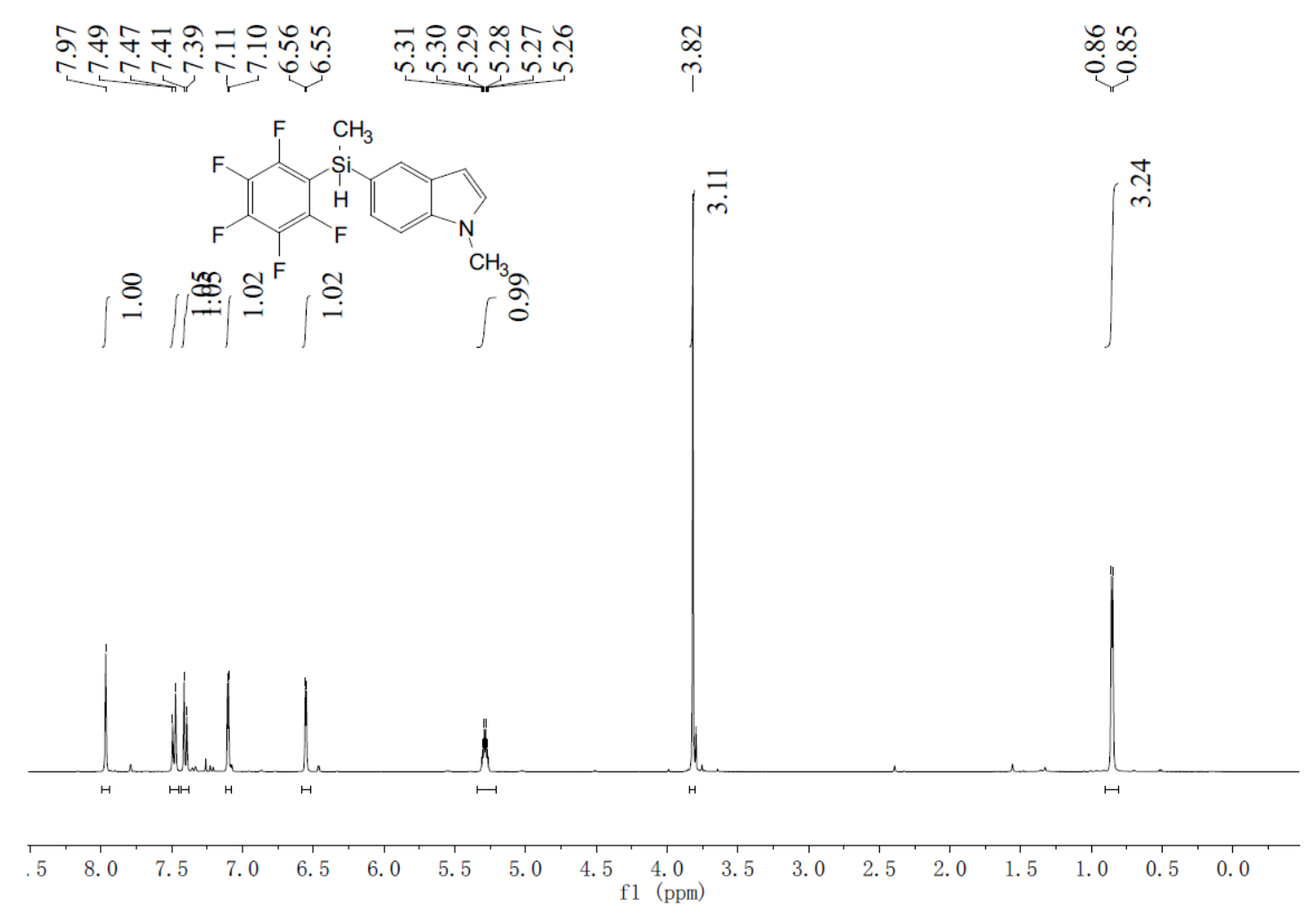

${ }^{1} \mathrm{H}$ NMR $\left(400 \mathrm{MHz}, \mathrm{CDCl}_{3}\right)$ of $\mathbf{2 e}$

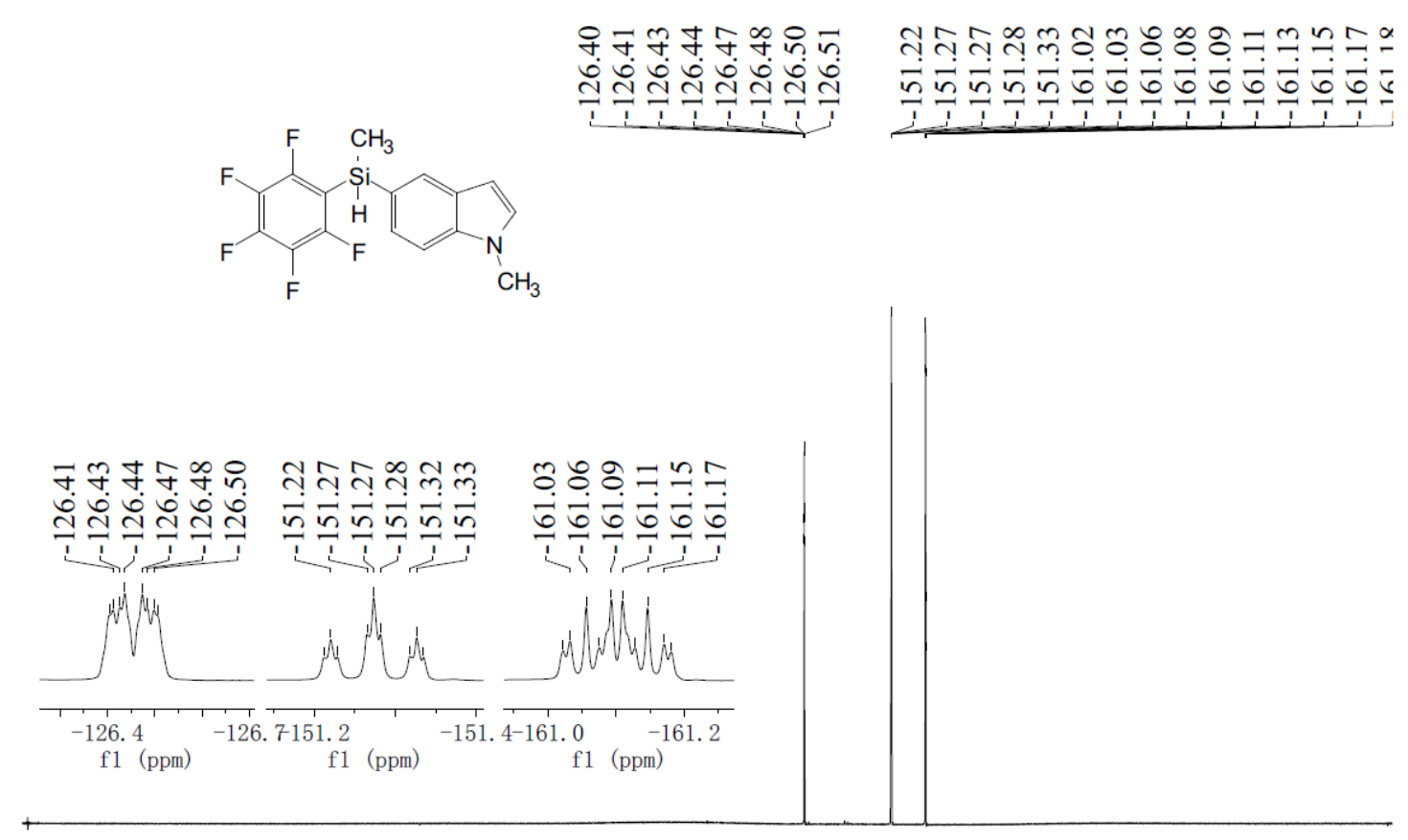

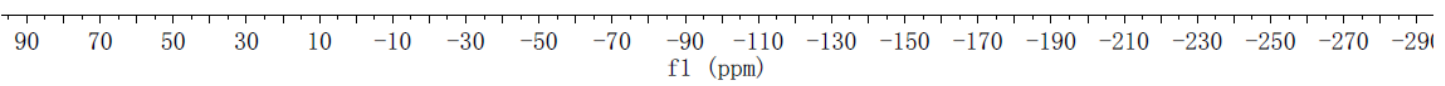

$\left.{ }^{19} \mathrm{~F} \mathrm{NMR} \mathrm{(376} \mathrm{MHz,} \mathrm{CDCl}_{3}\right)$ of $\mathbf{2 e}$ 
<smiles>Cn1ccc2cc(Sc3c(F)c(F)c(F)c(F)c3F)ccc21</smiles>

${ }^{13} \mathrm{C} \mathrm{NMR}\left(101 \mathrm{MHz}, \mathrm{CDCl}_{3}\right)$ of $\mathbf{2 e}$ 


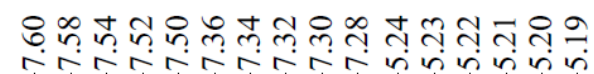

$\int_{0}^{2}$

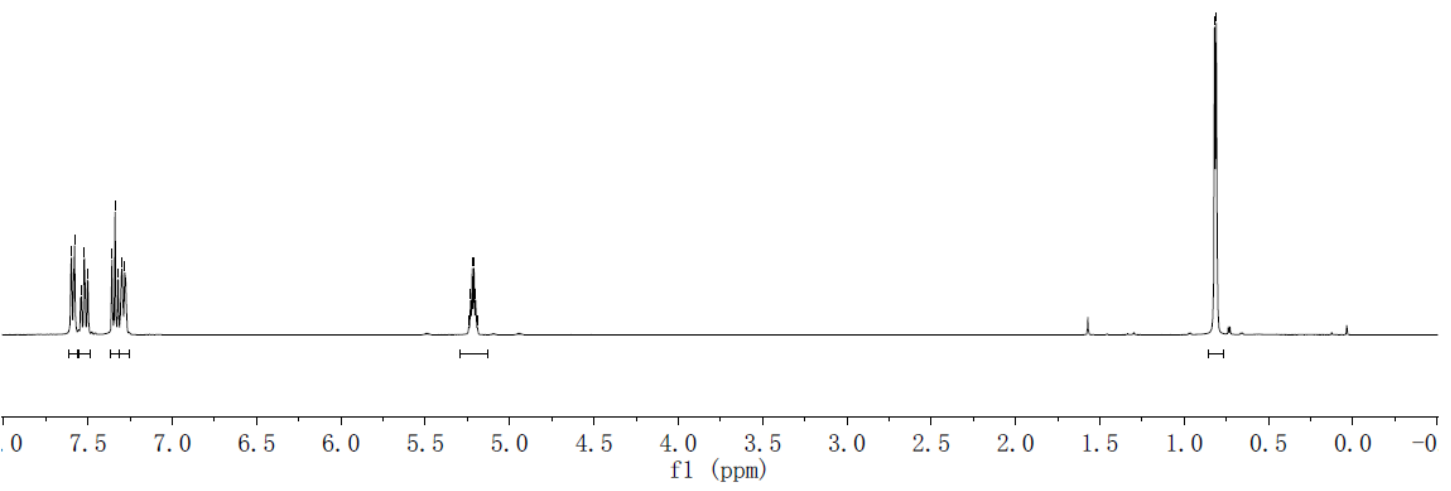

${ }^{1} \mathrm{H}$ NMR $\left(400 \mathrm{MHz}, \mathrm{CDCl}_{3}\right)$ of $\mathbf{2 f}$
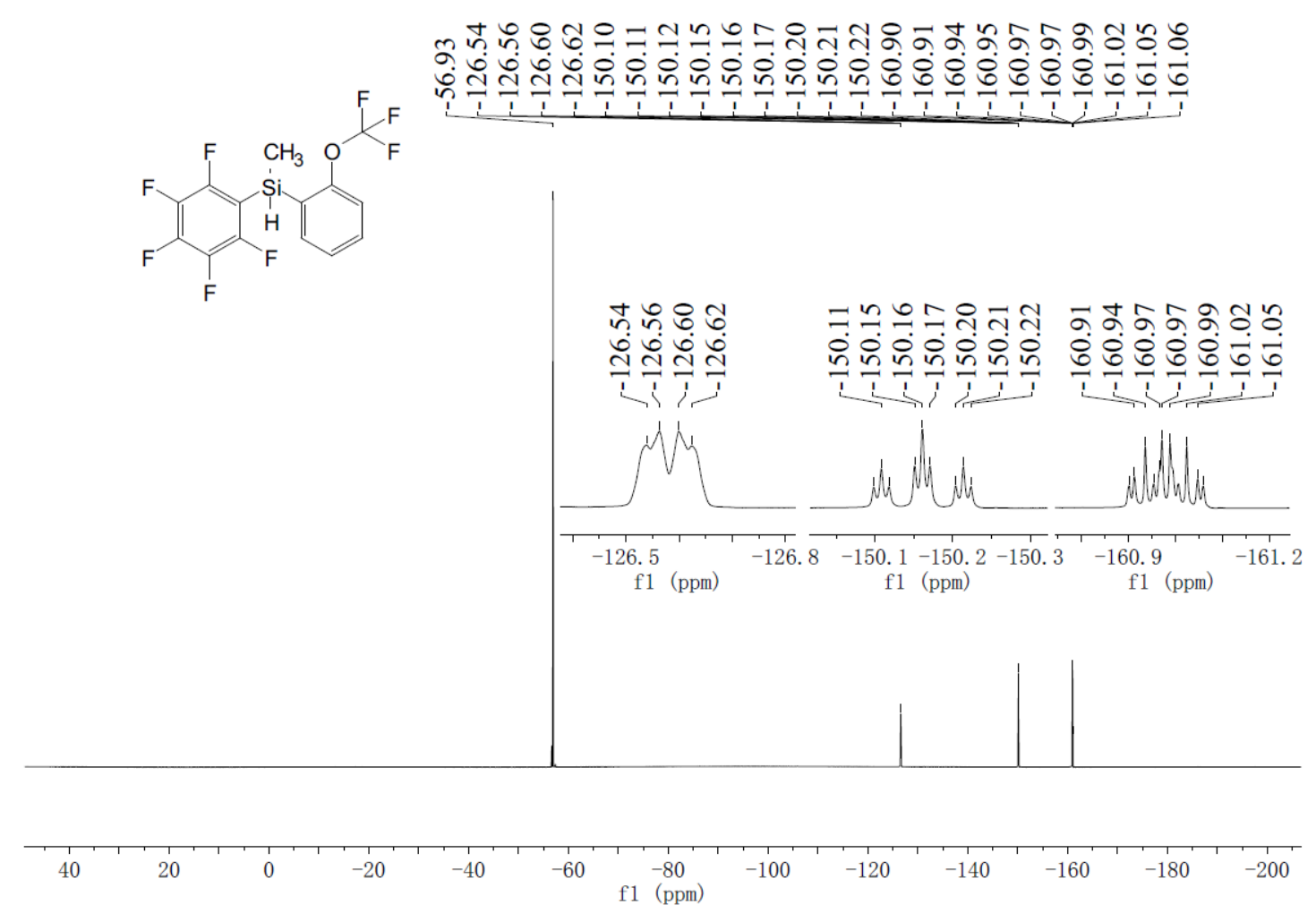

${ }^{19} \mathrm{~F} \mathrm{NMR} \mathrm{(376} \mathrm{MHz,} \mathrm{CDCl}_{3}$ ) of $\mathbf{2 f}$ 
<smiles>CC(OC(F)(F)F)c1ccccc1[Si](C)(C)c1c(F)c(F)c(F)c(F)c1F</smiles>

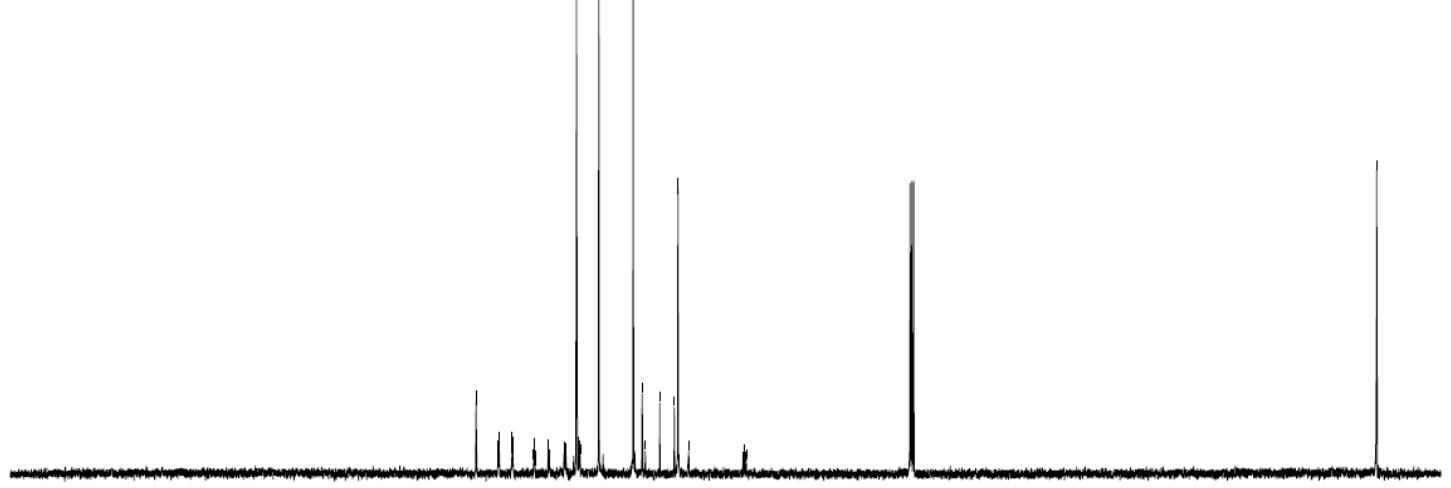

$\begin{array}{lllllllllllllllllllllllll}230 & 220 & 210 & 200 & 190 & 180 & 170 & 160 & 150 & 140 & 130 & 120 & 110 & 100 & 90 & 80 & 70 & 60 & 50 & 40 & 30 & 20 & 10 & 0 & -10\end{array}$ f1 (ppm)

${ }^{13} \mathrm{C}$ NMR $\left(101 \mathrm{MHz}, \mathrm{CDCl}_{3}\right)$ of $\mathbf{2 f}$ 

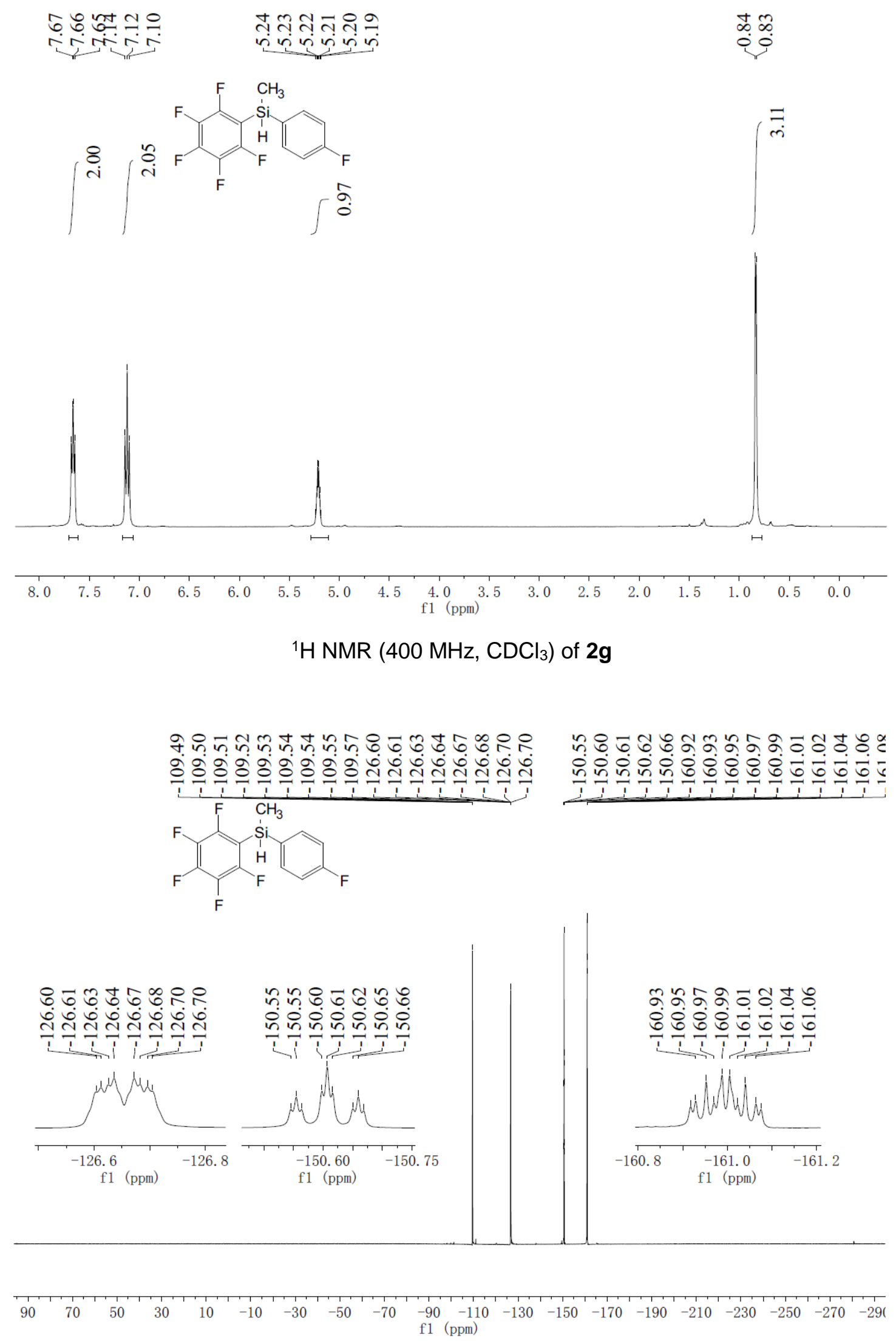

${ }^{19} \mathrm{~F} \mathrm{NMR}\left(376 \mathrm{MHz}, \mathrm{CDCl}_{3}\right)$ of $\mathbf{2 g}$ 


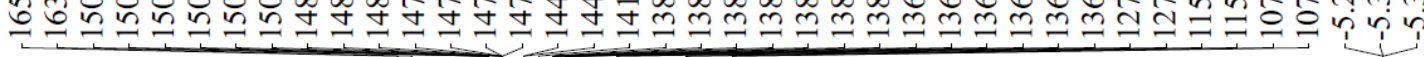<smiles>C[SH](c1ccc(F)cc1)c1c(F)c(F)c(F)c(F)c1C(C)(C)C</smiles>

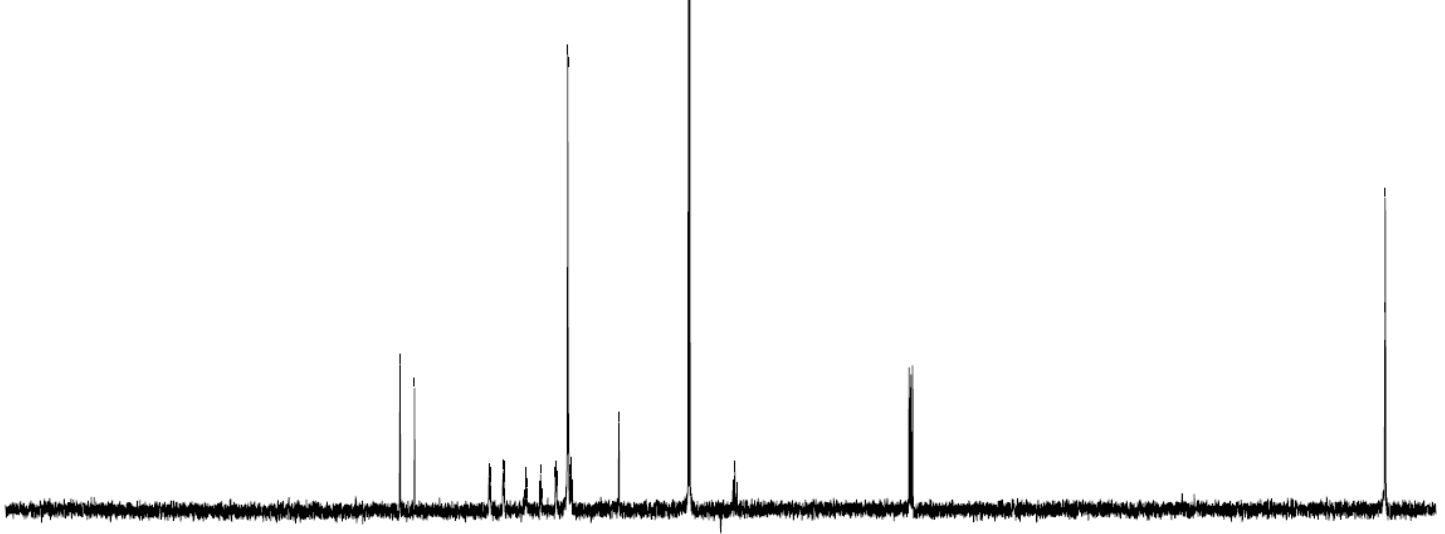

$\begin{array}{llllllllllllllllllllllllll}230 & 220 & 210 & 200 & 190 & 180 & 170 & 160 & 150 & 140 & 130 & 120 & 110 & 100 & 90 & 80 & 70 & 60 & 50 & 40 & 30 & 20 & 10 & 0 & -10\end{array}$

${ }^{13} \mathrm{C} \mathrm{NMR}\left(101 \mathrm{MHz}, \mathrm{CDCl}_{3}\right)$ of $\mathbf{2 g}$ 


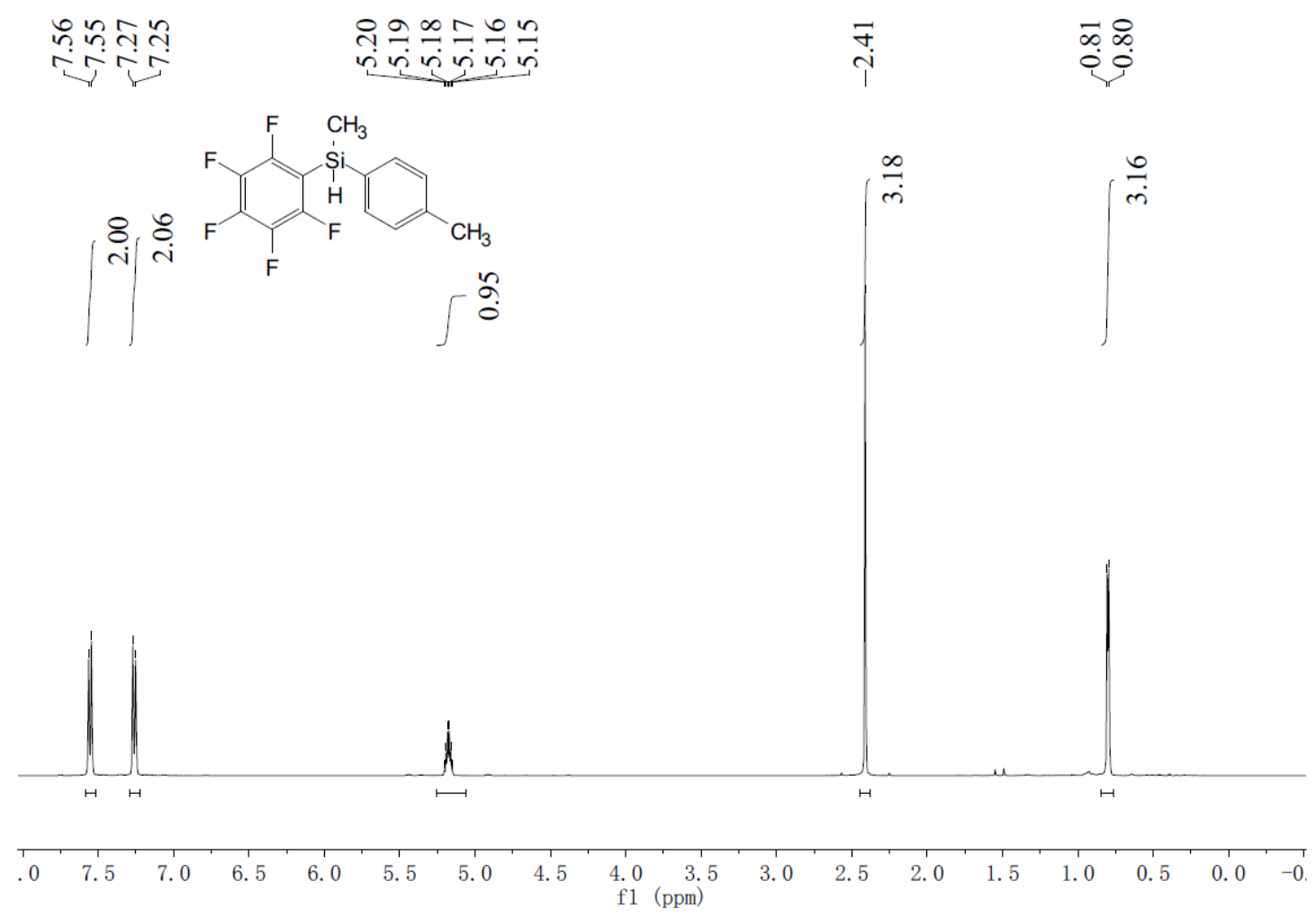

${ }^{1} \mathrm{H}$ NMR $\left(400 \mathrm{MHz}, \mathrm{CDCl}_{3}\right)$ of $\mathbf{2 h}$

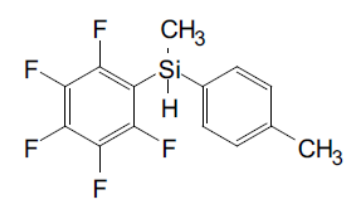

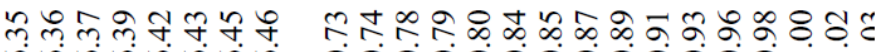

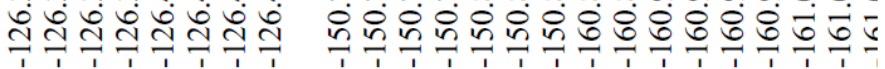

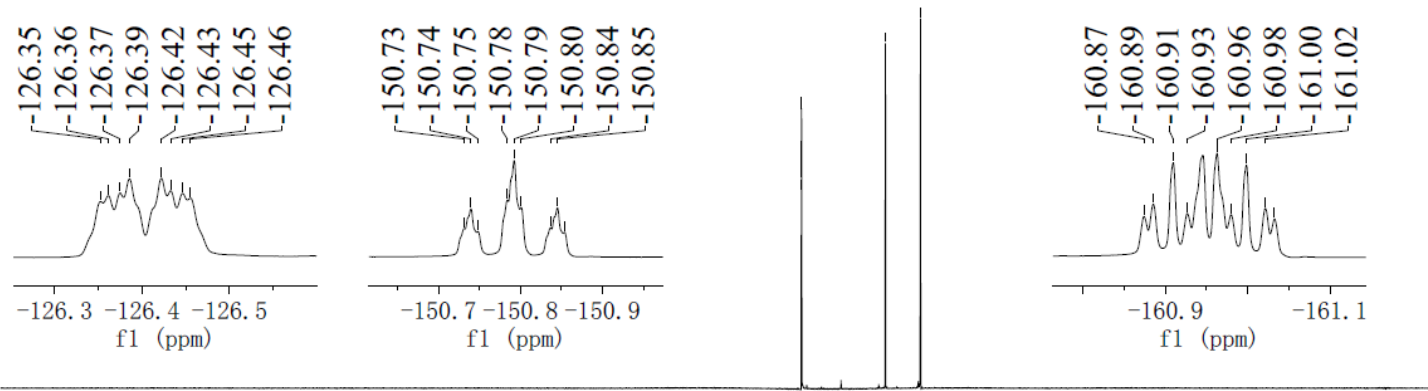

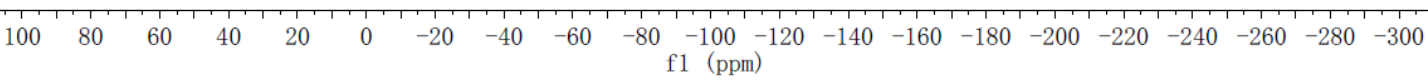

${ }^{19} \mathrm{~F} \mathrm{NMR}\left(376 \mathrm{MHz}, \mathrm{CDCl}_{3}\right)$ of $\mathbf{2 h}$ 
<smiles>Cc1ccc(Sc2c(F)c(F)c(F)c(F)c2F)cc1</smiles>

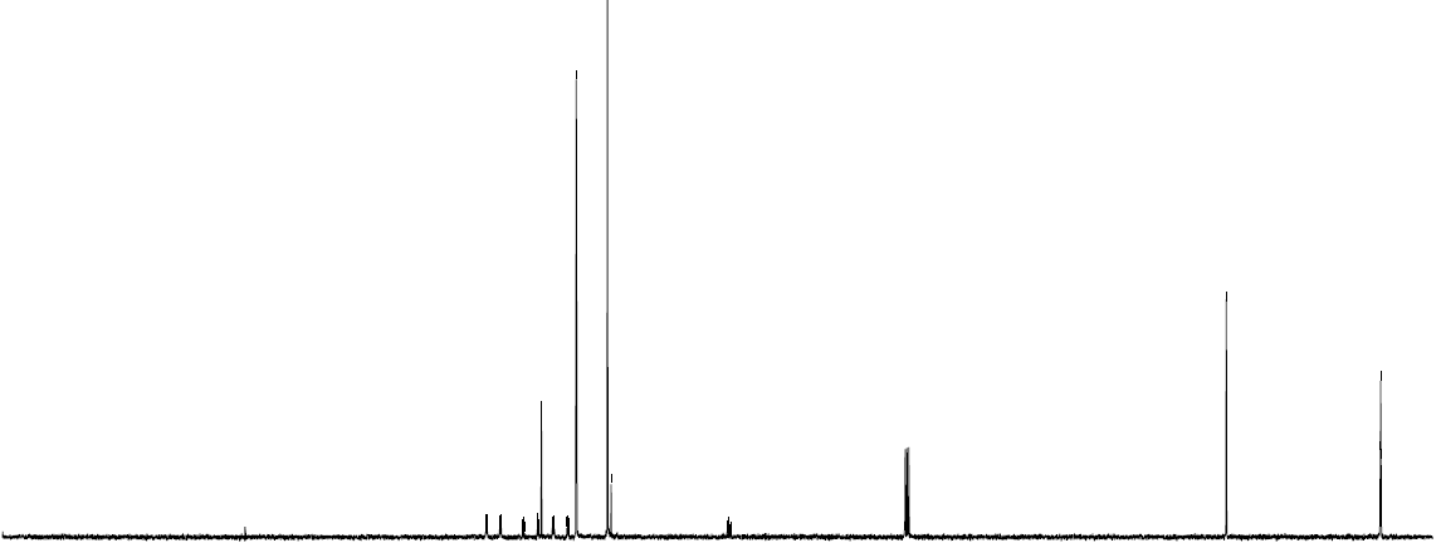

$\begin{array}{lllllllllllllllllllllllll}230 & 220 & 210 & 200 & 190 & 180 & 170 & 160 & 150 & 140 & 130 & 120 & 110 & 100 & 90 & 80 & 70 & 60 & 50 & 40 & 30 & 20 & 10 & 0 & -10\end{array}$ f1 (ppm)

${ }^{13} \mathrm{C} \mathrm{NMR}\left(101 \mathrm{MHz}, \mathrm{CDCl}_{3}\right)$ of $\mathbf{2 h}$ 


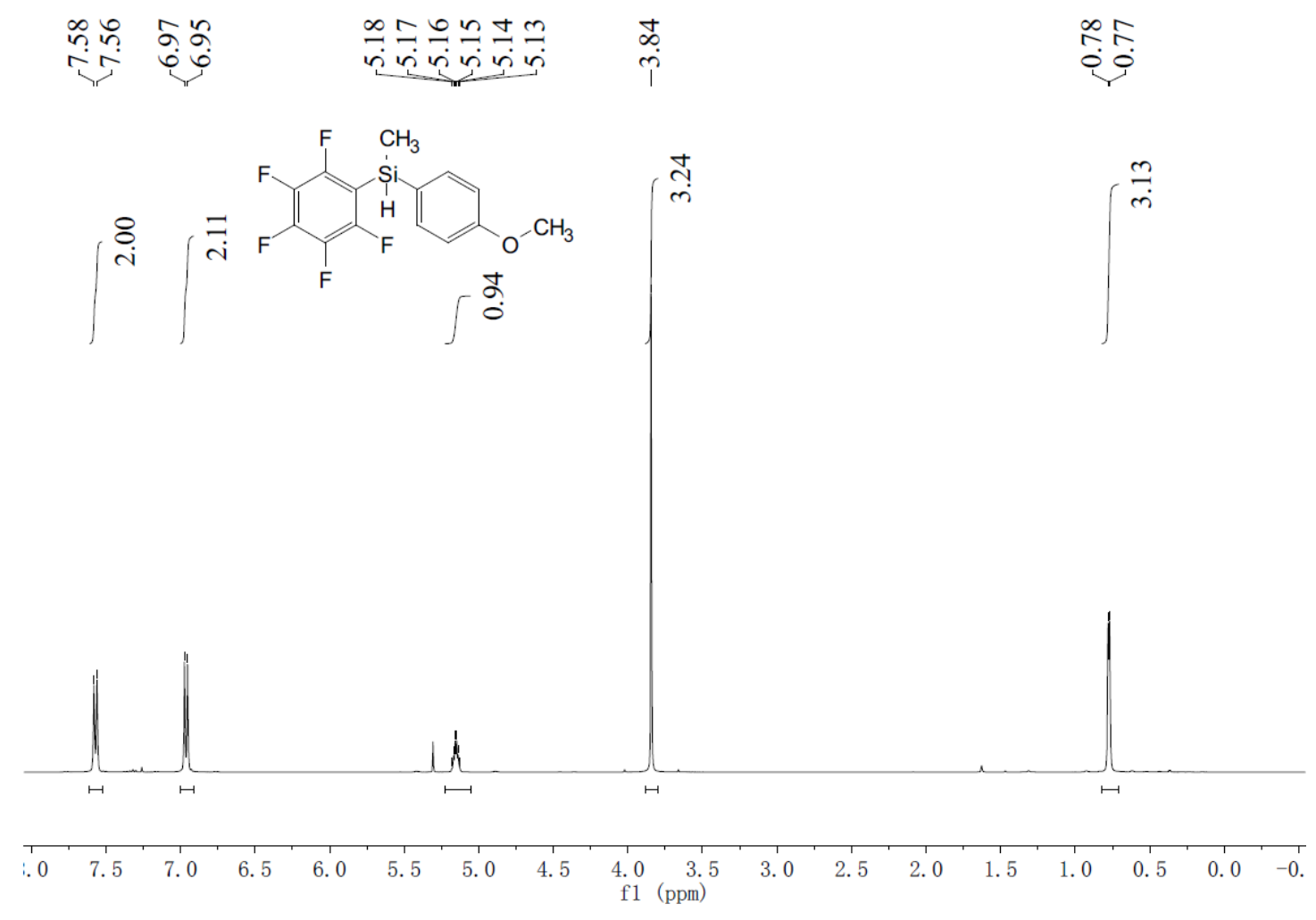

${ }^{1} \mathrm{H}$ NMR $\left(400 \mathrm{MHz}, \mathrm{CDCl}_{3}\right)$ of $\mathbf{2} \mathbf{i}$

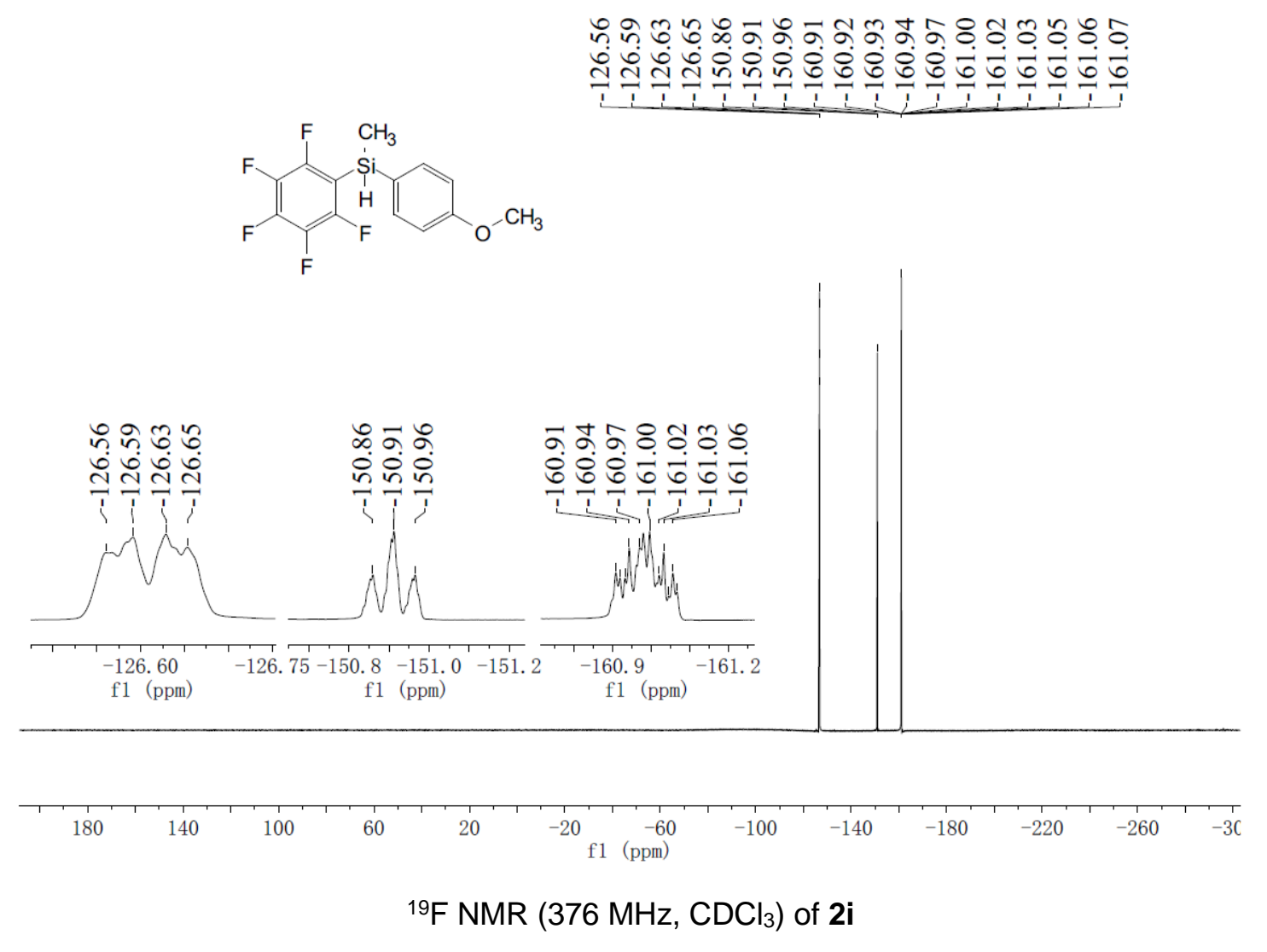




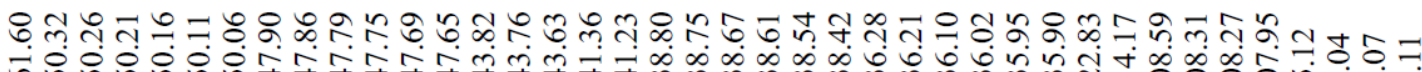

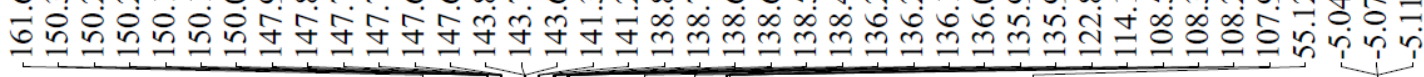<smiles>COc1ccc([Si](C)(C)c2c(F)c(F)c(F)c(F)c2F)cc1</smiles>

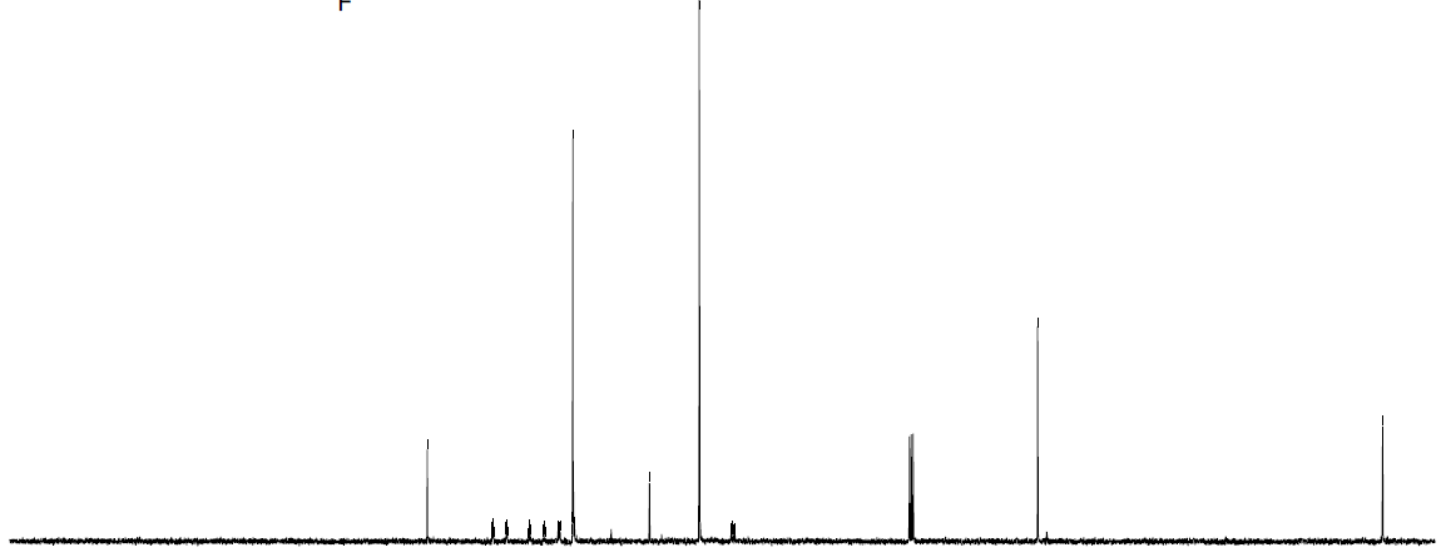

$\begin{array}{lllllllllllllllllllllllllll}230 & 220 & 210 & 200 & 190 & 180 & 170 & 160 & 150 & 140 & 130 & 120 & 110 & 100 & 90 & 80 & 70 & 60 & 50 & 40 & 30 & 20 & 10 & 0 & -10\end{array}$ ${ }^{13} \mathrm{C}$ NMR $\left(101 \mathrm{MHz}, \mathrm{CDCl}_{3}\right)$ of $\mathbf{2} \mathbf{i}$ 


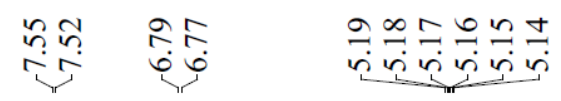

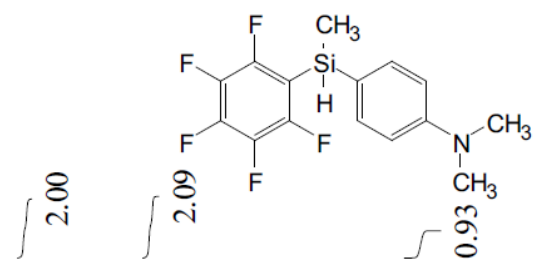

iิ

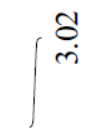

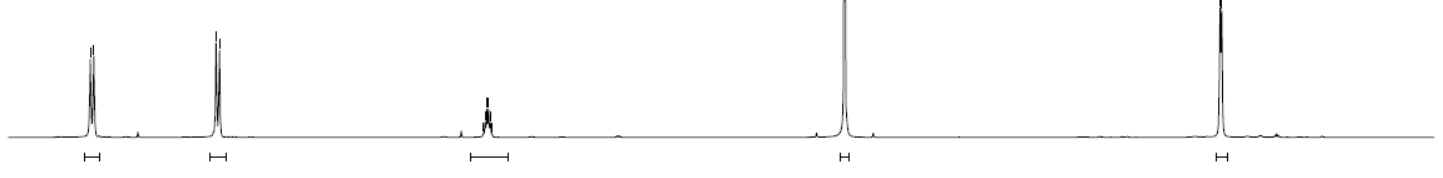

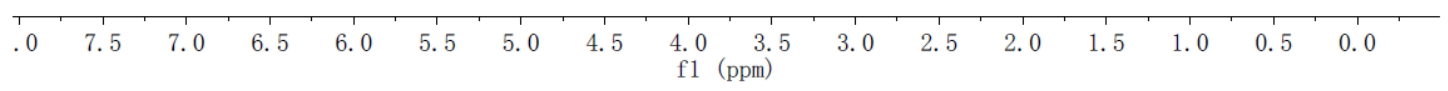

${ }^{1} \mathrm{H}$ NMR $\left(400 \mathrm{MHz}, \mathrm{CDCl}_{3}\right)$ of $\mathbf{2 j}$<smiles>CN(C)c1ccc([Si](C)(C)c2c(F)c(F)c(F)c(F)c2F)cc1</smiles>
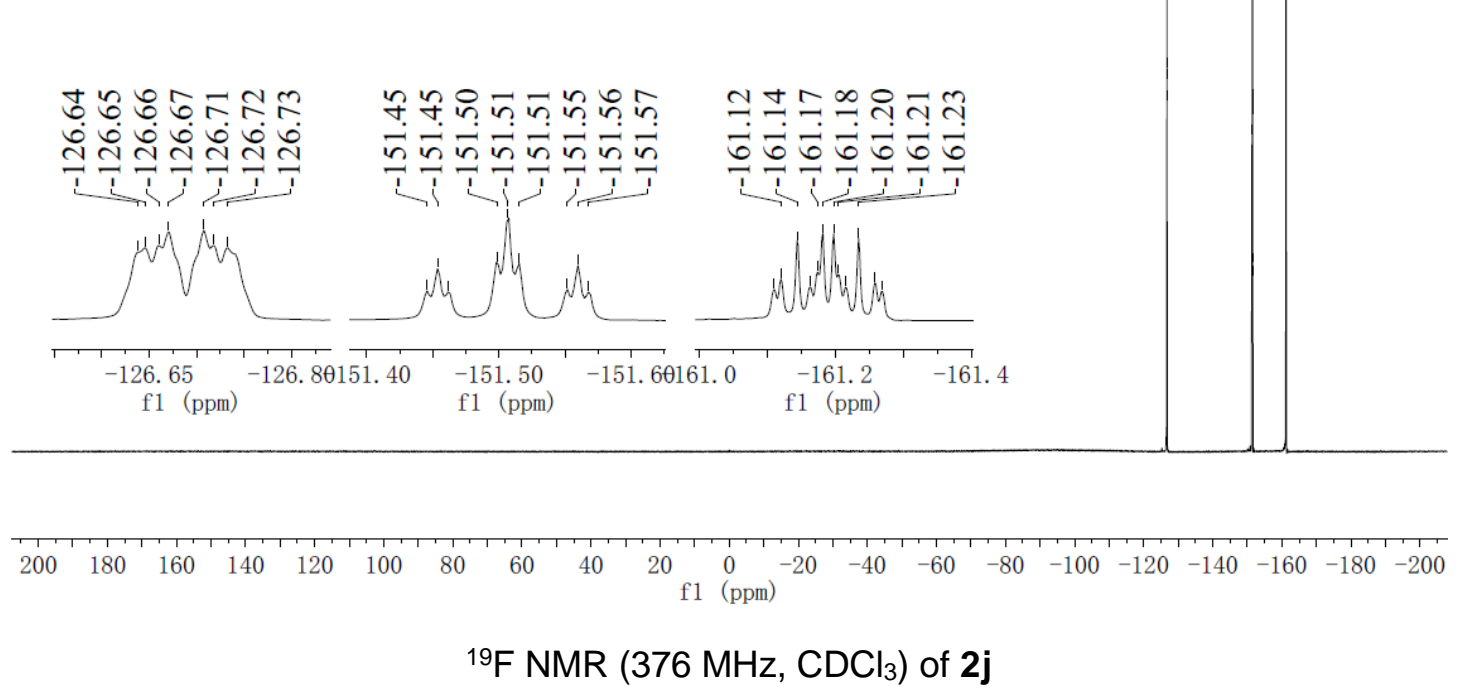

S67 


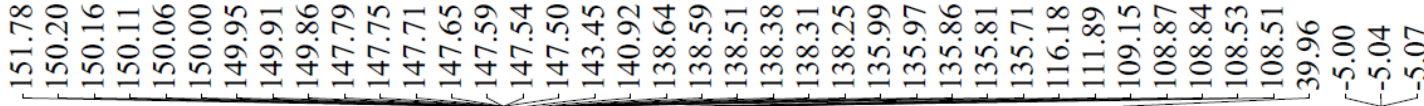<smiles>CN(C)c1ccc([SiH](C)c2c(F)c(F)c(F)c(F)c2F)cc1</smiles>

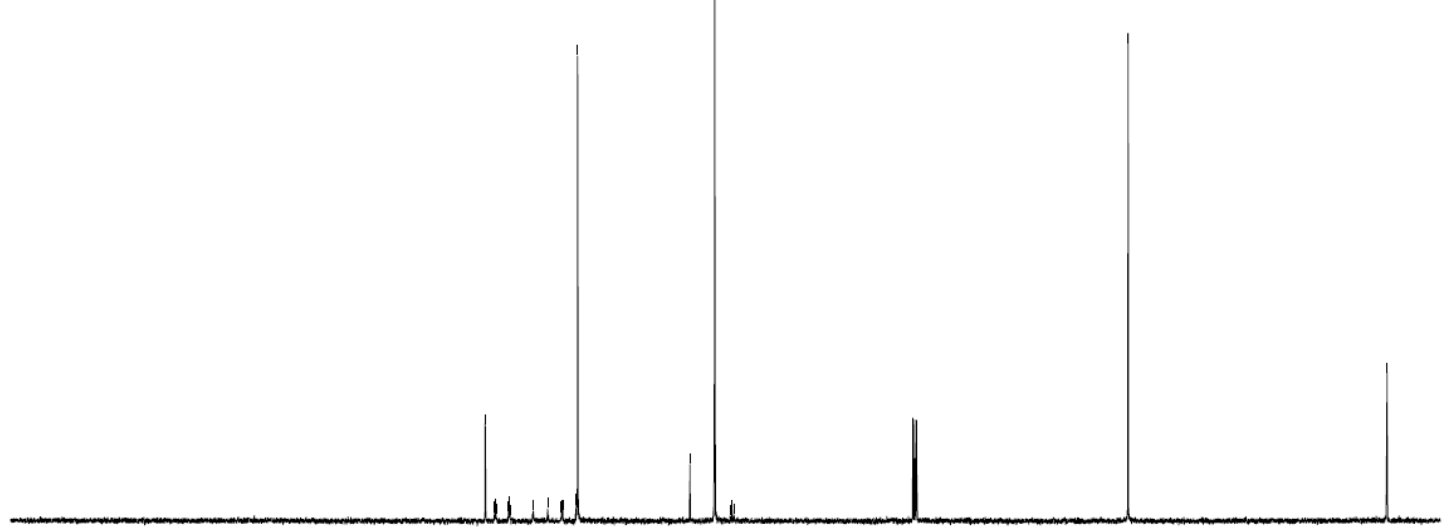

$\begin{array}{llllllllllllllllllllllllllll}230 & 220 & 210 & 200 & 190 & 180 & 170 & 160 & 150 & 140 & 130 & 120 & 110 & 100 & 90 & 80 & 70 & 60 & 50 & 40 & 30 & 20 & 10 & 0 & -10\end{array}$ ${ }^{13} \mathrm{C}$ NMR (101 MHz, $\mathrm{CDCl}_{3}$ ) of $\mathbf{2 j}$ 


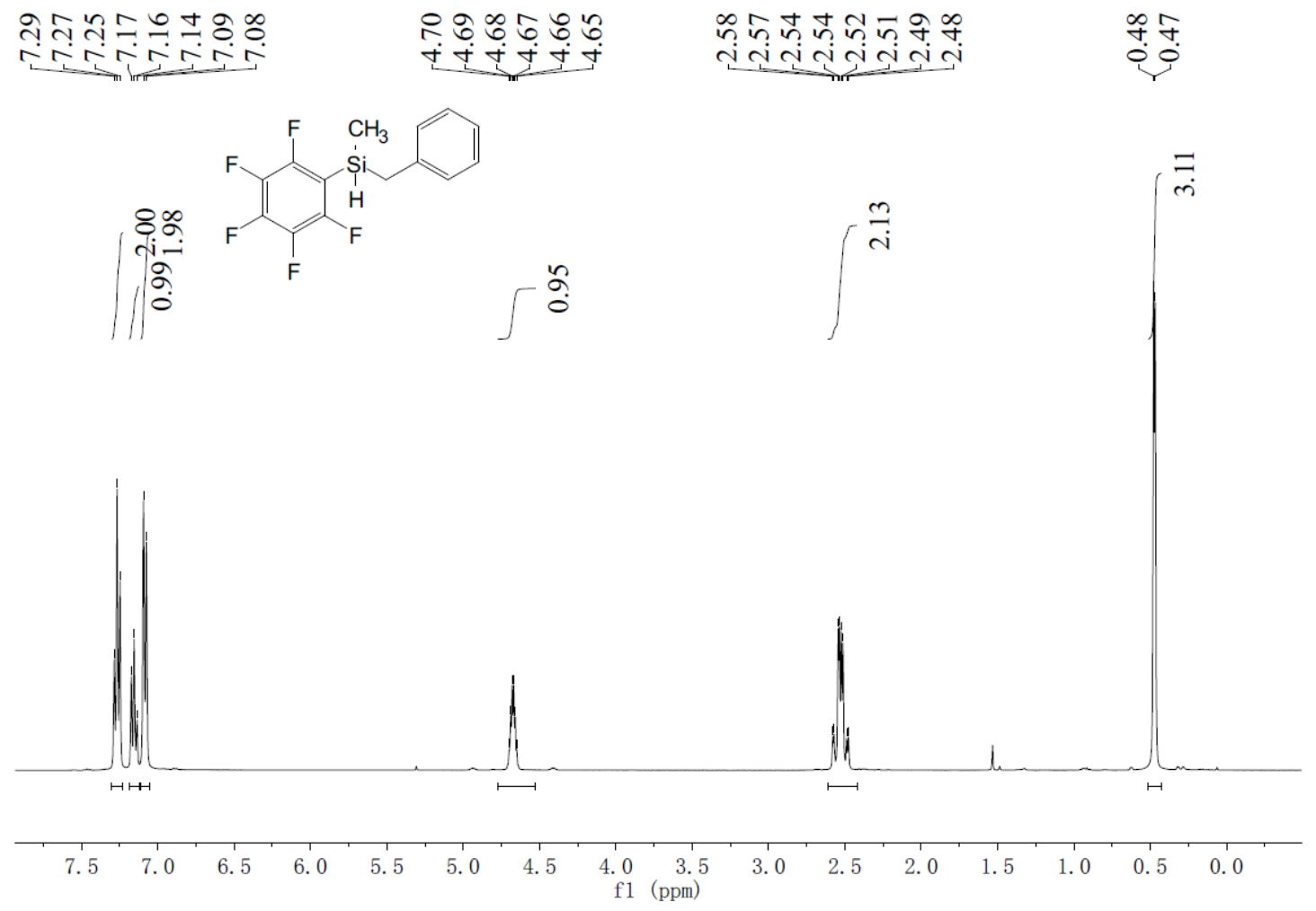

${ }^{1} \mathrm{H}$ NMR $\left(400 \mathrm{MHz}, \mathrm{CDCl}_{3}\right)$ of $\mathbf{2} \mathbf{k}$

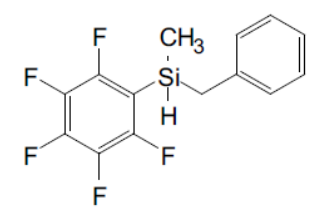

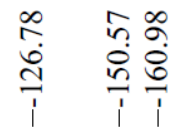

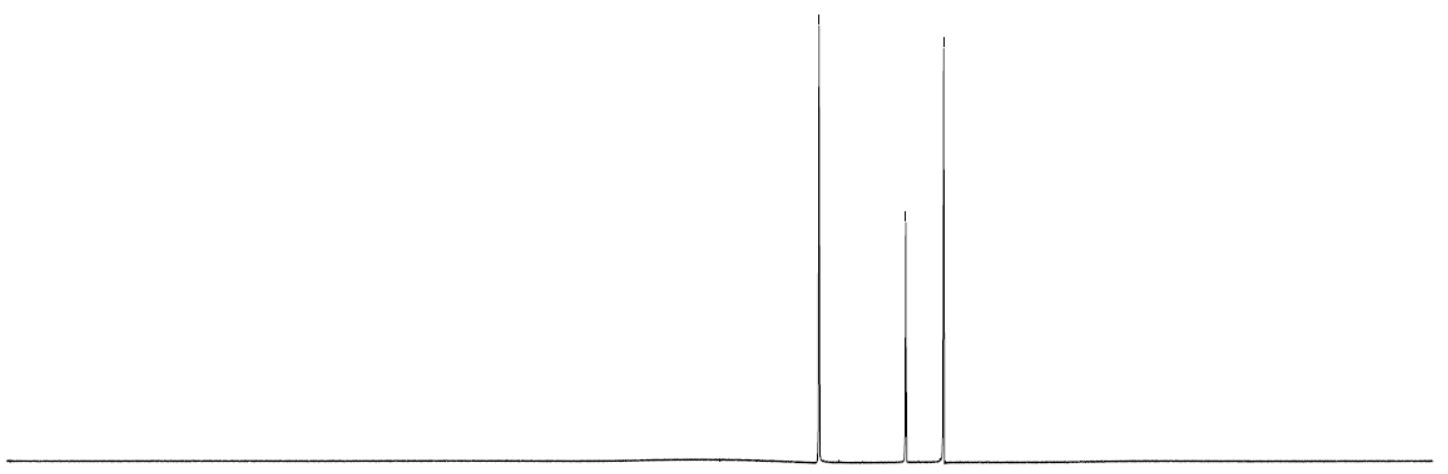

\begin{tabular}{llllllllllllllllllll}
\hline 90 & 70 & 50 & 30 & 10 & -10 & -30 & -50 & -70 & -90 & -110 & -130 & -150 & -170 & -190 & -210 & -230 & -250 & -270 & -29(
\end{tabular} $\left.{ }^{19} \mathrm{~F} \mathrm{NMR} \mathrm{(376} \mathrm{MHz,} \mathrm{CDCl}_{3}\right)$ of $\mathbf{2 k}$ 


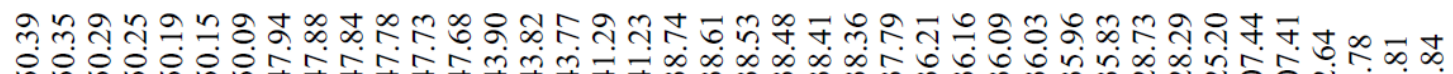

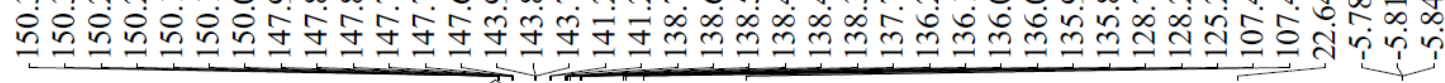<smiles>C[Si](Cc1ccccc1)c1c(F)c(F)c(F)c(F)c1F</smiles>

$\begin{array}{lllllllllllllllllllllllllll}230 & 220 & 210 & 200 & 190 & 180 & 170 & 160 & 150 & 140 & 130 & 120 & 110 & 100 & 90 & 80 & 70 & 60 & 50 & 40 & 30 & 20 & 10 & 0 & -10 \\ \mathrm{f} 1 & (\mathrm{ppm}) & & \end{array}$ $\left.{ }^{13} \mathrm{C} \mathrm{NMR} \mathrm{(101} \mathrm{MHz,} \mathrm{CDCl}_{3}\right)$ of $\mathbf{2 k}$ 


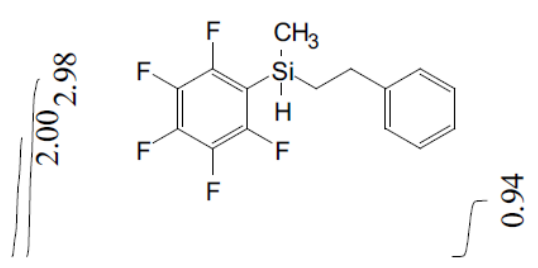
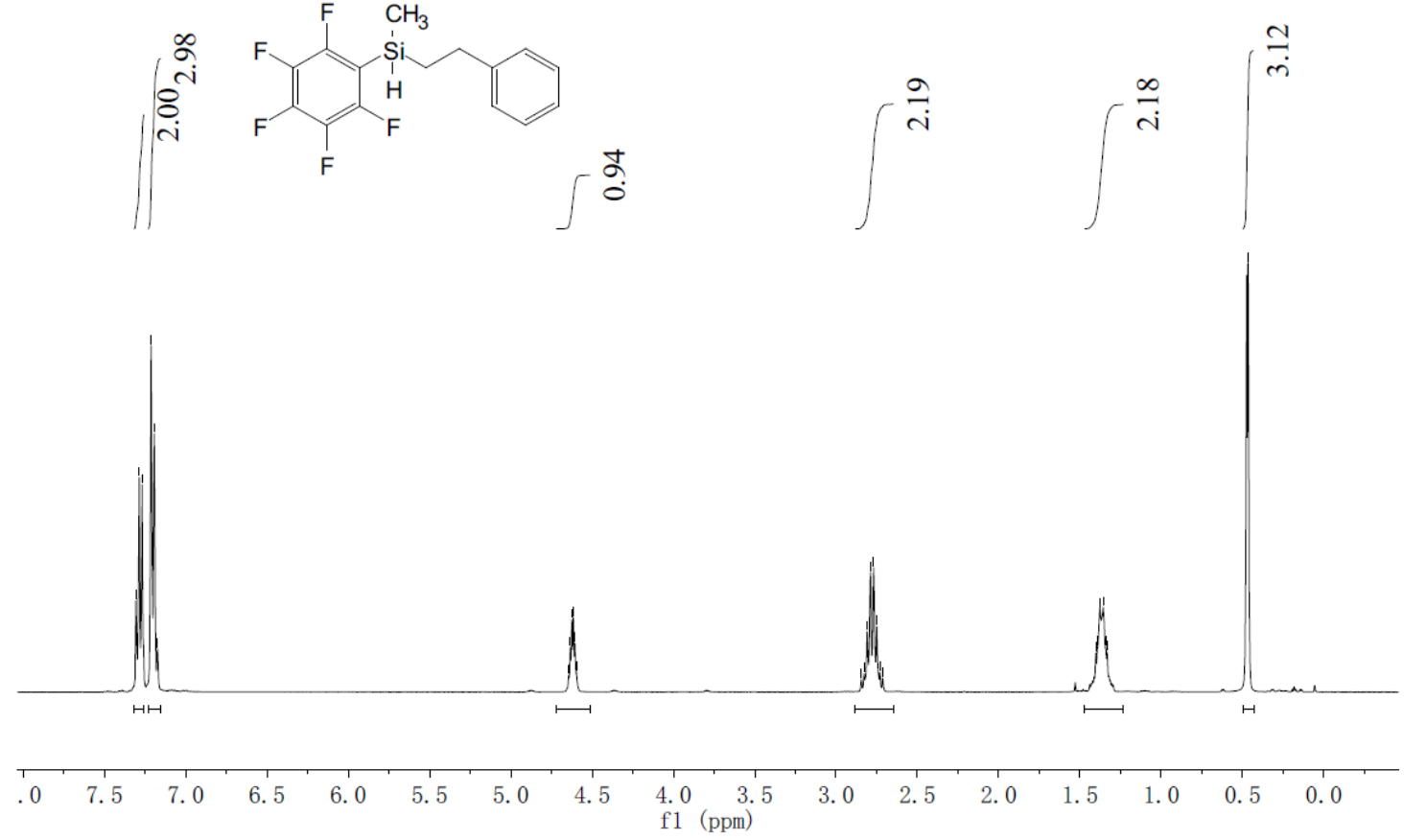

${ }^{1} \mathrm{H} \mathrm{NMR}\left(400 \mathrm{MHz}, \mathrm{CDCl}_{3}\right)$ of $\mathbf{2 l}$

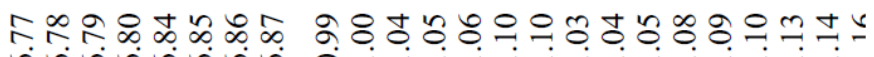

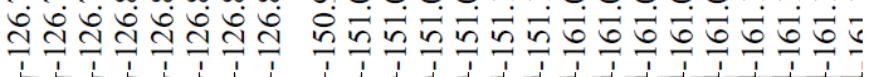<smiles>C[Si](CCc1ccccc1)c1c(F)c(F)c(F)c(F)c1F</smiles>

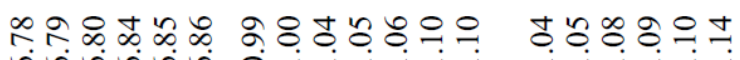
원워

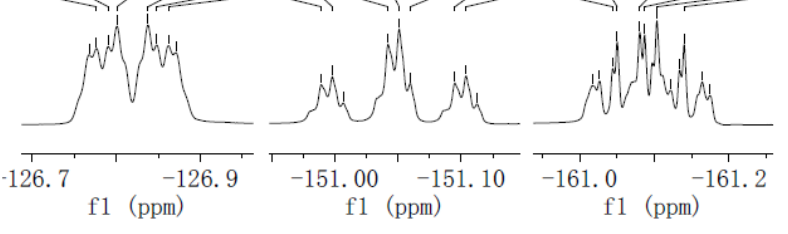

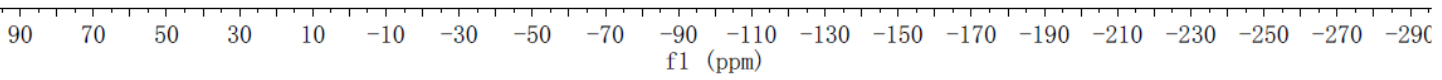
$\left.{ }^{19} \mathrm{~F} \mathrm{NMR} \mathrm{(376} \mathrm{MHz,} \mathrm{CDCl}_{3}\right)$ of $\mathbf{2 l}$ 


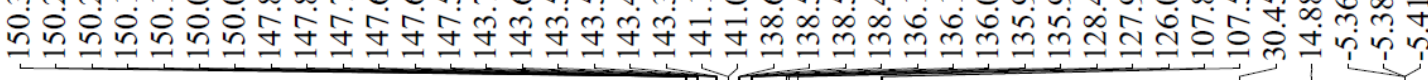<smiles>C[Si](CCc1ccccc1)c1c(F)c(F)c(F)c(F)c1F</smiles>
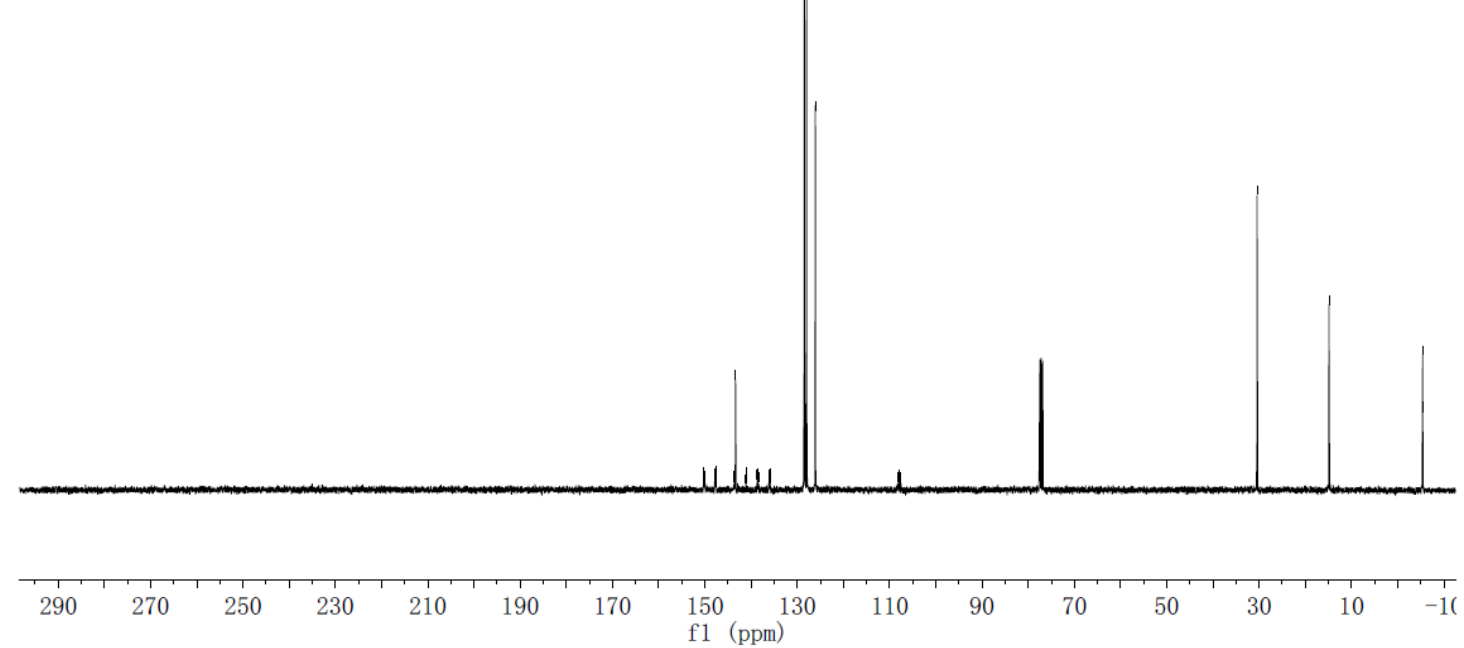

${ }^{13} \mathrm{C}$ NMR $\left(101 \mathrm{MHz}, \mathrm{CDCl}_{3}\right)$ of $\mathbf{2 l}$ 


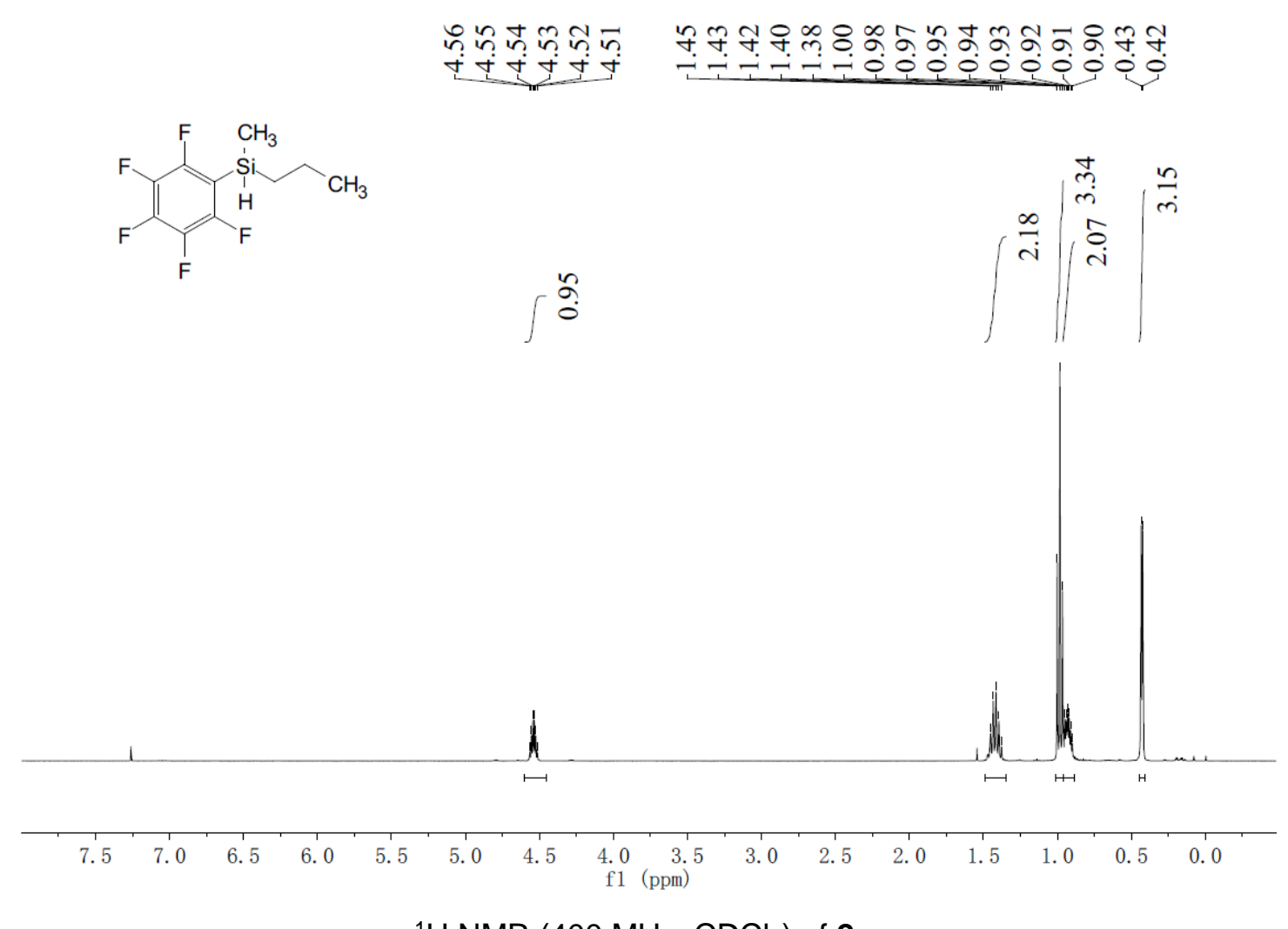

${ }^{1} \mathrm{H}$ NMR (400 MHz, $\left.\mathrm{CDCl}_{3}\right)$ of $\mathbf{2 m}$

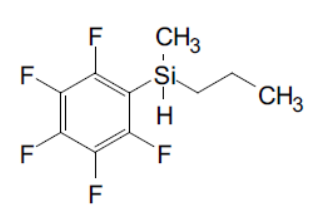

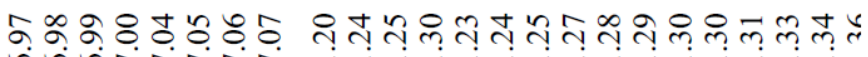

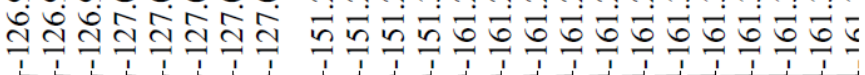

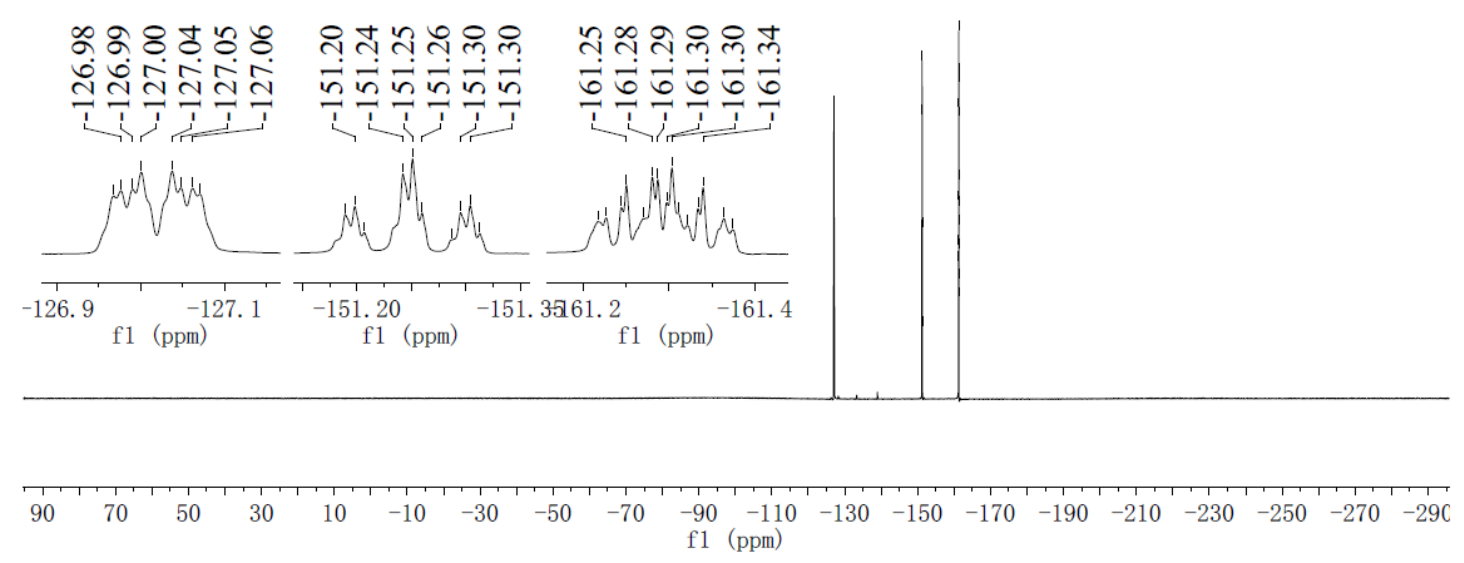

$\left.{ }^{19} \mathrm{~F} \mathrm{NMR} \mathrm{(376} \mathrm{MHz,} \mathrm{CDCl}_{3}\right)$ of $\mathbf{2 m}$ 


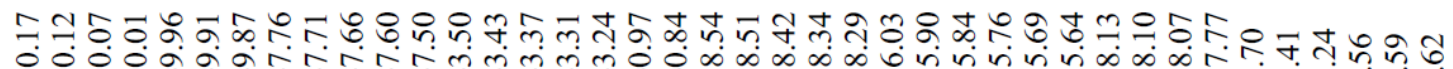

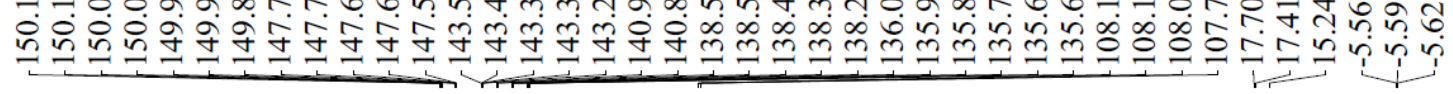<smiles>CCC[SiH](C)c1c(F)c(F)c(F)c(F)c1F</smiles>

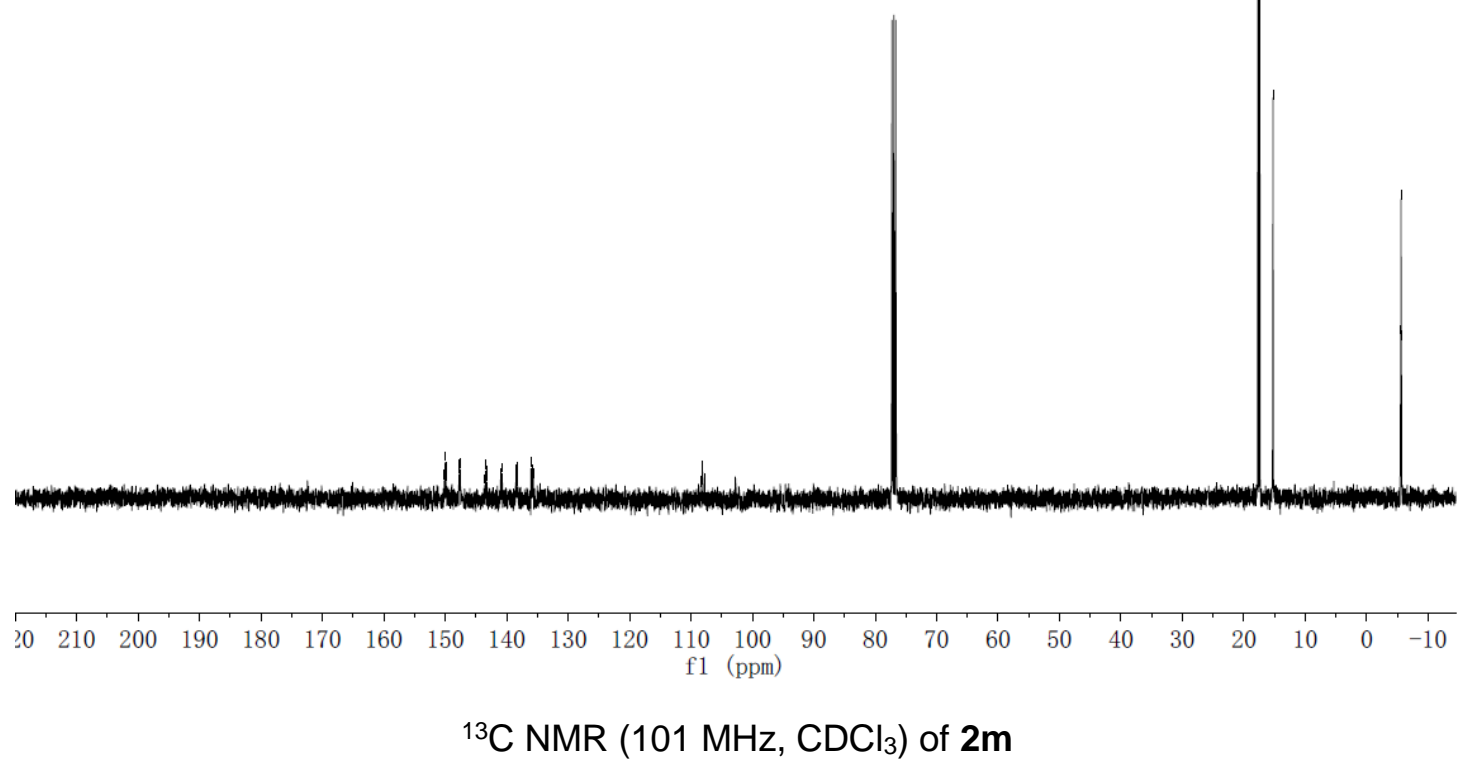


<smiles>C[Si](C)c1c(F)c(F)c(F)c(F)c1F</smiles>

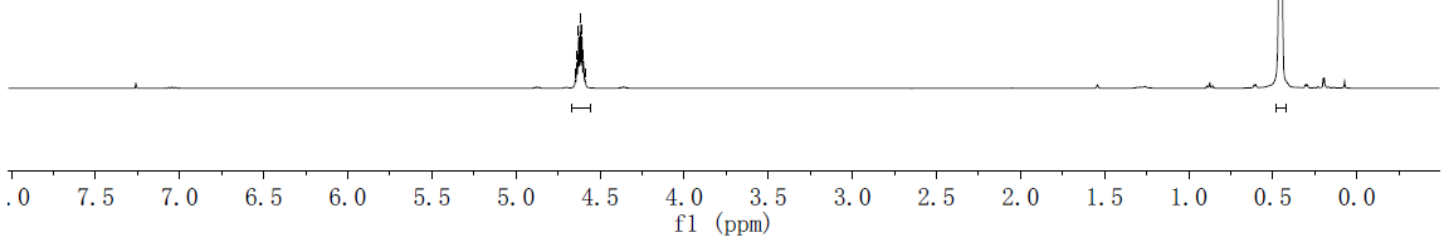

${ }^{1} \mathrm{H} \mathrm{NMR}\left(400 \mathrm{MHz}, \mathrm{CDCl}_{3}\right)$ of $\mathbf{2 n}$<smiles>C[Si](C)c1c(F)c(F)c(F)c(F)c1F</smiles>

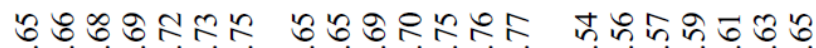

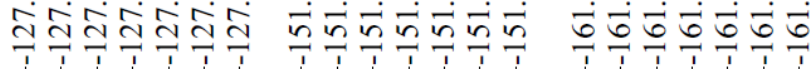
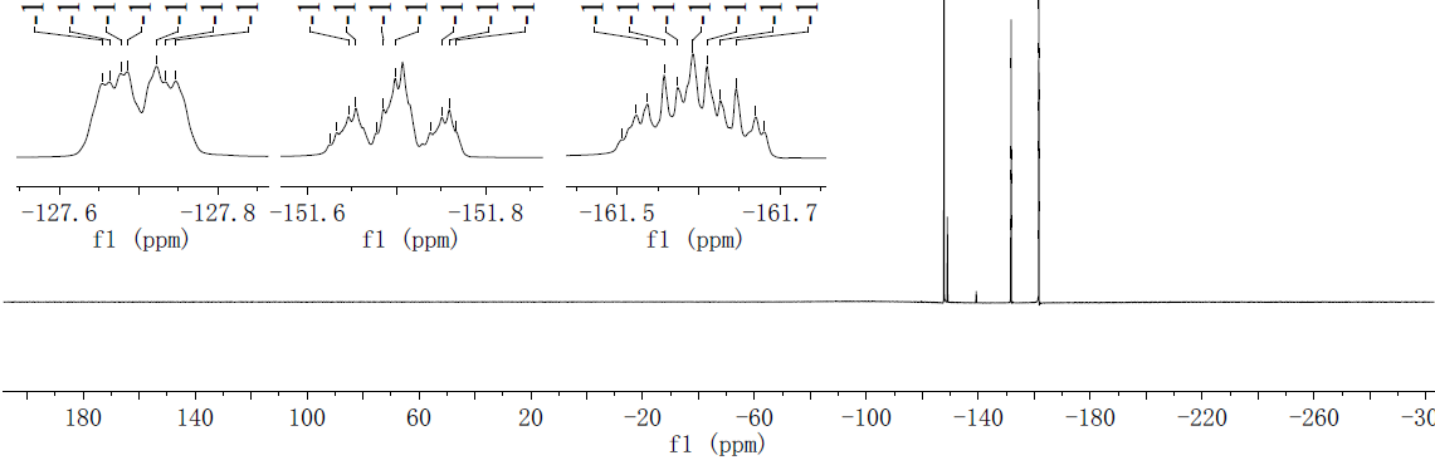

${ }^{19} \mathrm{~F} \mathrm{NMR}\left(376 \mathrm{MHz}, \mathrm{CDCl}_{3}\right)$ of $\mathbf{2 n}$ 


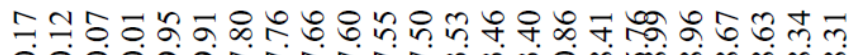

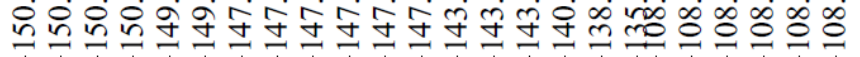<smiles>C[SiH](C)c1c(F)c(F)c(F)c(F)c1F</smiles>

${ }^{13} \mathrm{C}$ NMR $\left(101 \mathrm{MHz}, \mathrm{CDCl}_{3}\right)$ of $\mathbf{2 n}$ 


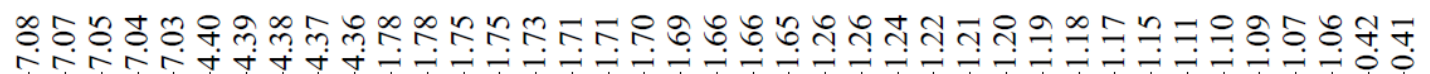

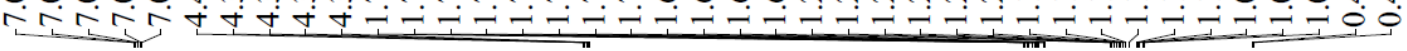<smiles>CCc1cc(F)c(F)c([Si](C)(C2CCCCC2)C2CCCCC2)c1F</smiles>

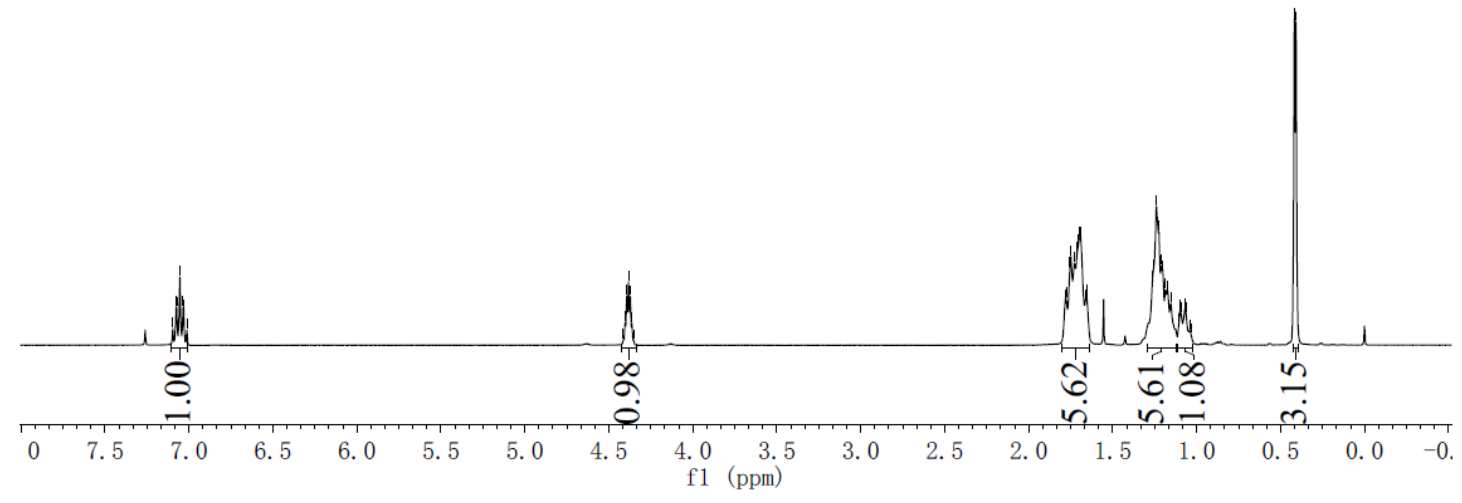

${ }^{1} \mathrm{H}$ NMR $\left(400 \mathrm{MHz}, \mathrm{CDCl}_{3}\right)$ of $\mathbf{2 0}$<smiles>C[Si](c1c(F)c(F)cc(F)c1F)C1CCCCC1</smiles>

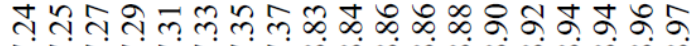

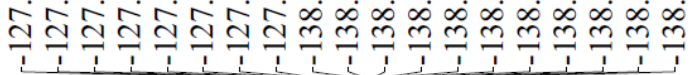

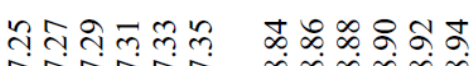

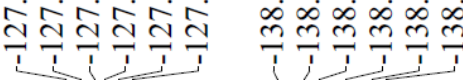

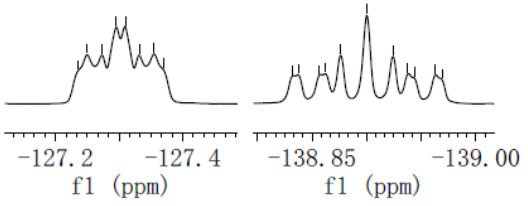

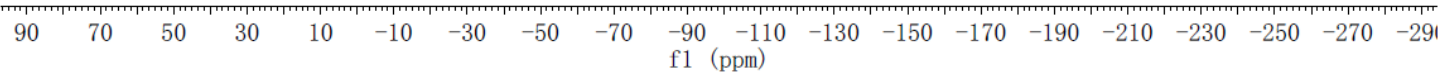

${ }^{19} \mathrm{~F} \mathrm{NMR}\left(376 \mathrm{MHz}, \mathrm{CDCl}_{3}\right)$ of 20 


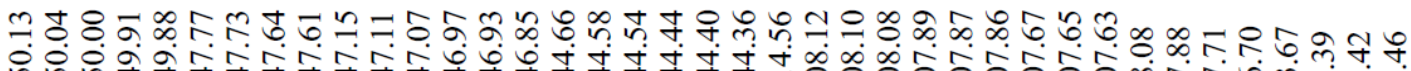

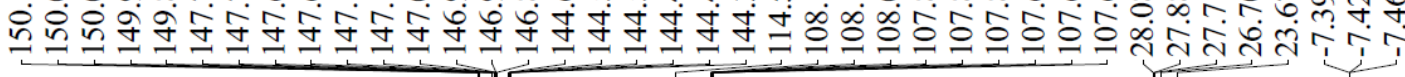<smiles>C[Si](c1c(F)c(F)cc(F)c1F)C1CCCCC1</smiles>

${ }^{13} \mathrm{C} \mathrm{NMR}\left(101 \mathrm{MHz}, \mathrm{CDCl}_{3}\right)$ of 20 
<smiles>C[Si](C)c1c(F)c(F)cc(F)c1F</smiles>

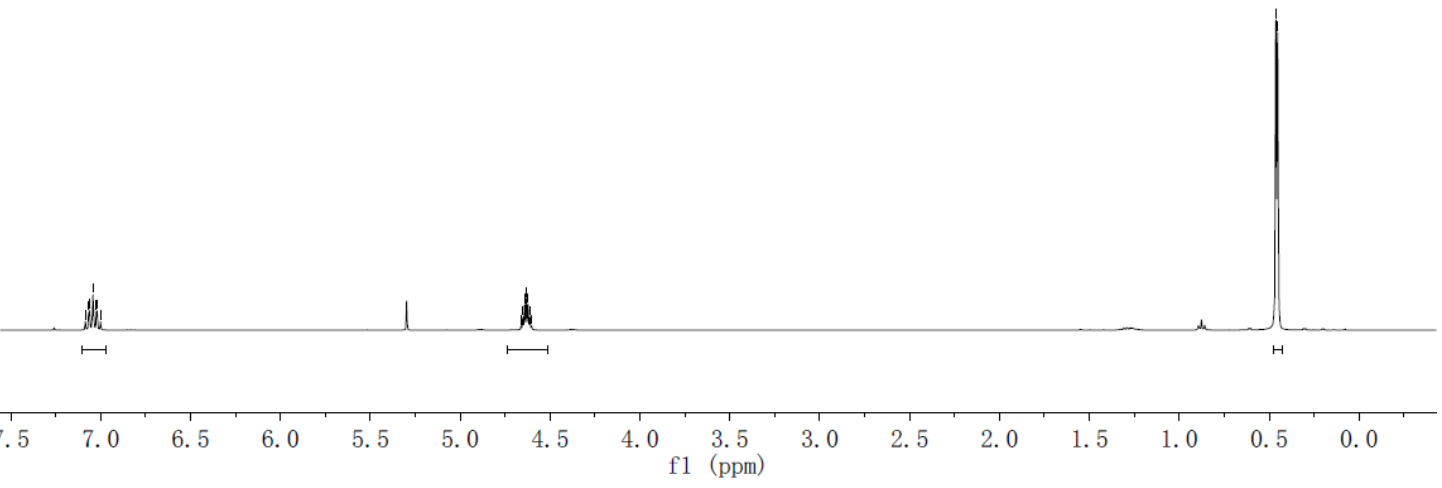

${ }^{1} \mathrm{H} \mathrm{NMR}\left(400 \mathrm{MHz}, \mathrm{CDCl}_{3}\right)$ of $\mathbf{2 p}$

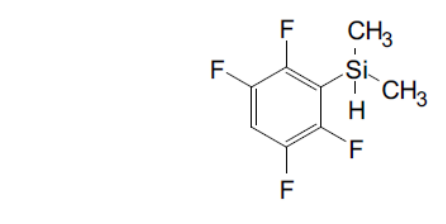

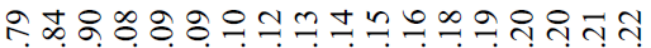

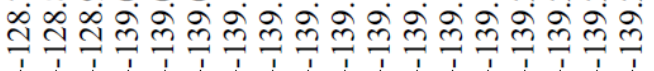

용ㅇ.

$\stackrel{i}{i} \stackrel{i}{i}$
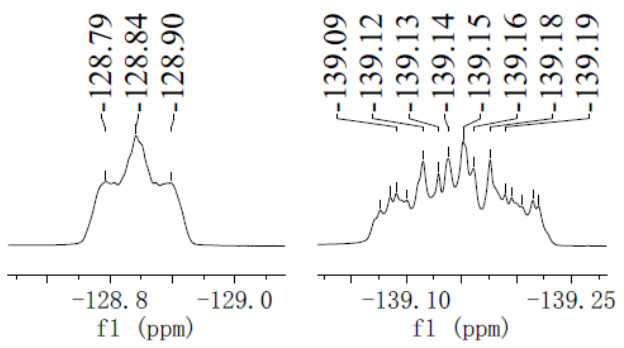

$\begin{array}{llllllllllllllllllllllllllllll}90 & 70 & 50 & 30 & 10 & -10 & -30 & -50 & -70 & -90 & -110 & -130 & -150 & -170 & -190 & -210 & -230 & -250 & -270 & -290 & -31\end{array}$

$\left.{ }^{19} \mathrm{~F} \mathrm{NMR} \mathrm{(376} \mathrm{MHz,} \mathrm{CDCl}_{3}\right)$ of $2 p$ 


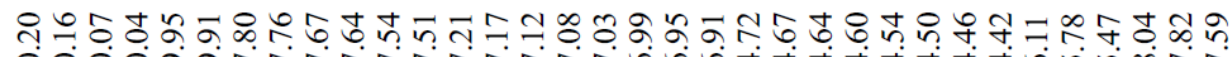

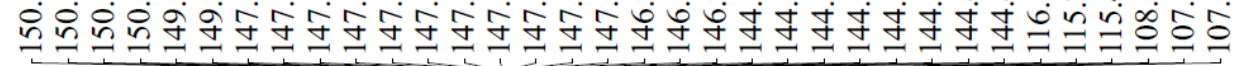<smiles>C[SiH](C)c1c(F)c(F)cc(F)c1F</smiles>

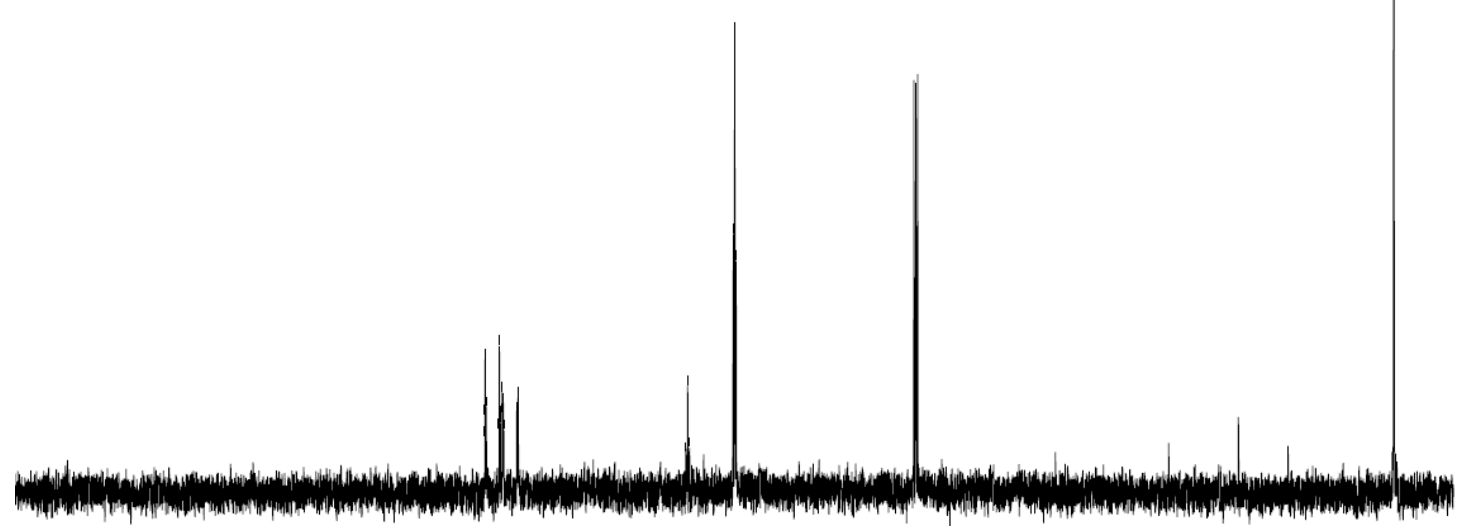

$\begin{array}{llllllllllllllllllllllll}220 & 210 & 200 & 190 & 180 & 170 & 160 & 150 & 140 & 130 & 120 & 110 & 100 & 90 & 80 & 70 & 60 & 50 & 40 & 30 & 20 & 10 & 0 & -10\end{array}$ ${ }^{13} \mathrm{C}$ NMR $\left(101 \mathrm{MHz}, \mathrm{CDCl}_{3}\right)$ of $\mathbf{2 p}$ 


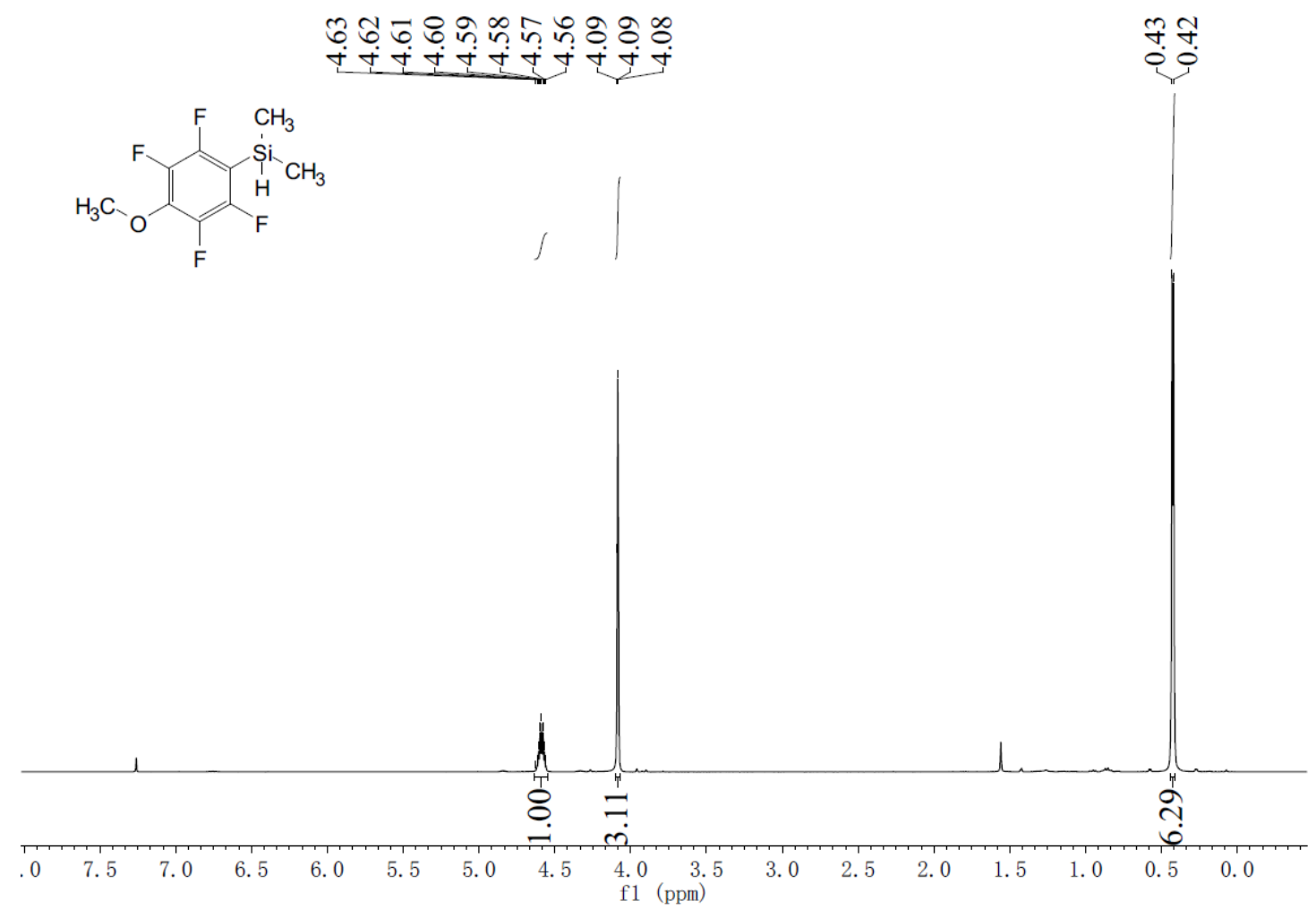

${ }^{1} \mathrm{H} \mathrm{NMR}\left(400 \mathrm{MHz}, \mathrm{CDCl}_{3}\right)$ of $\mathbf{2 q}$<smiles>COC1C(F)=C(F)C(C)=C(F)C1C</smiles>

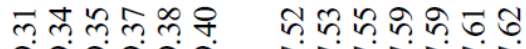

จิำำ

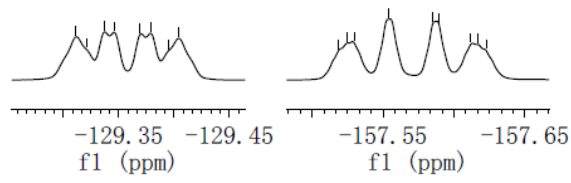

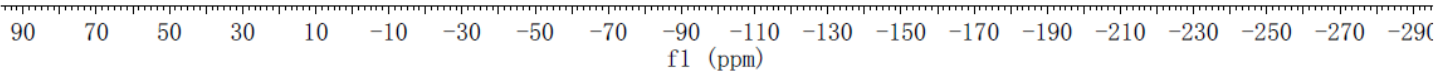

${ }^{19} \mathrm{~F} \mathrm{NMR}\left(376 \mathrm{MHz}, \mathrm{CDCl}_{3}\right)$ of $\mathbf{2 q}$ 


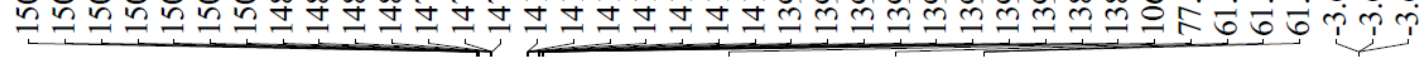<smiles>COc1c(F)c(F)c([Si](C)(C)C)c(F)c1F</smiles>

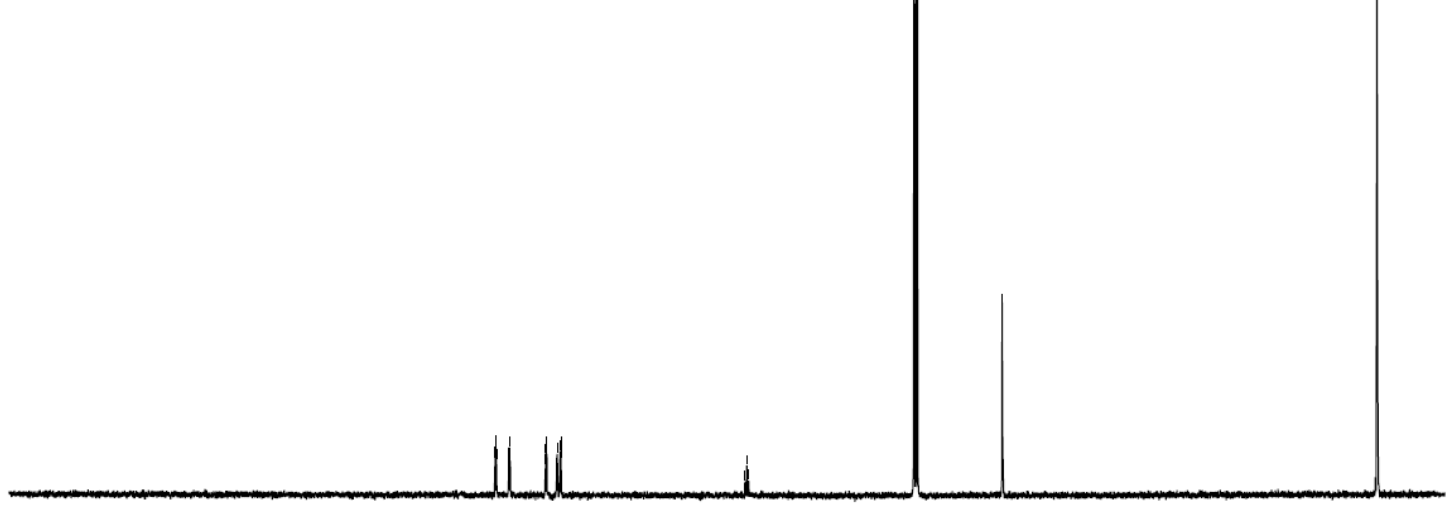

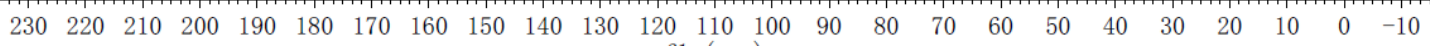
f1 (ppm)

${ }^{13} \mathrm{C} \mathrm{NMR}\left(101 \mathrm{MHz}, \mathrm{CDCl}_{3}\right)$ of $\mathbf{2 q}$ 


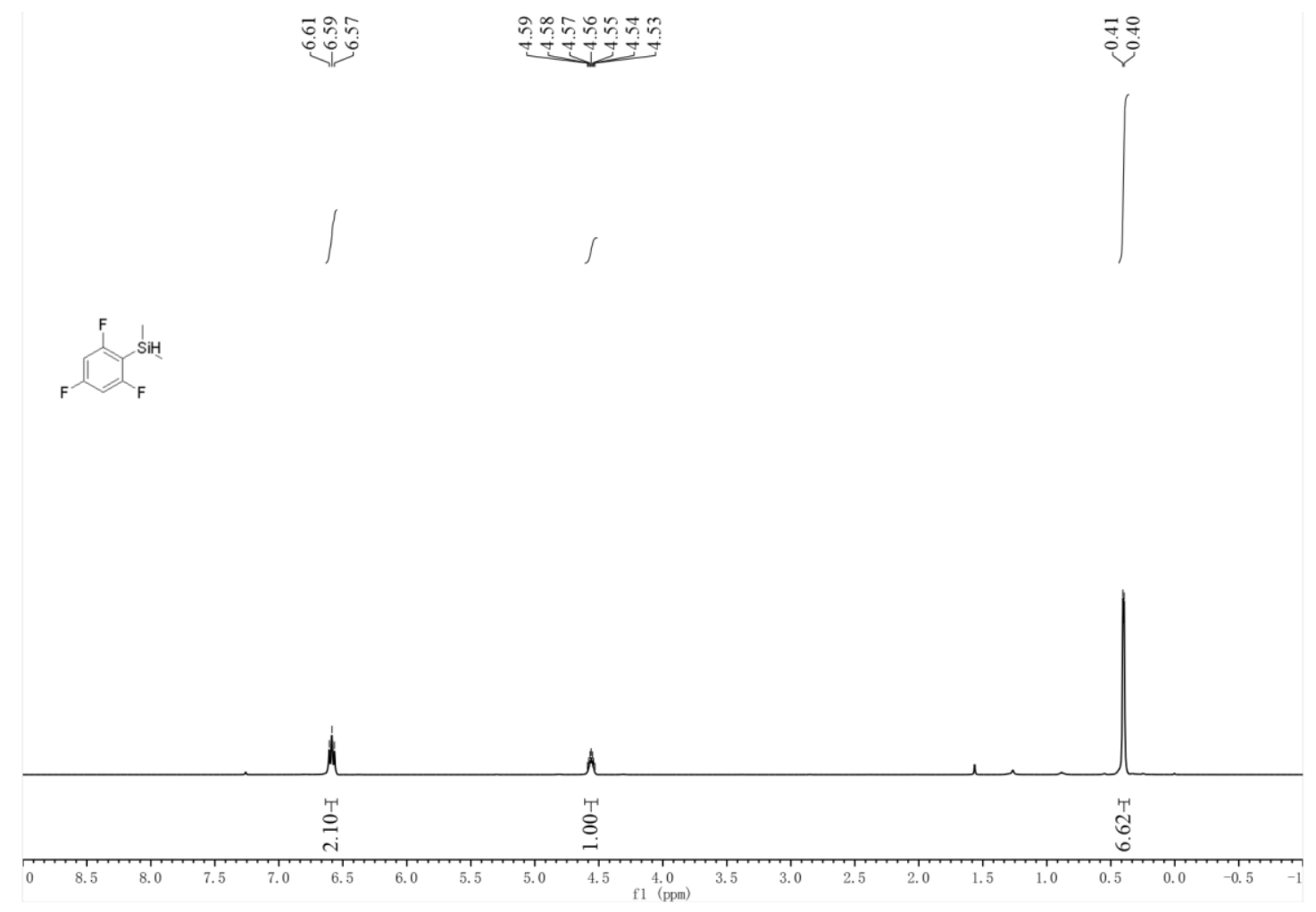

${ }^{1} \mathrm{H}$ NMR $\left(400 \mathrm{MHz}, \mathrm{CDCl}_{3}\right)$ of $\mathbf{2 r}$

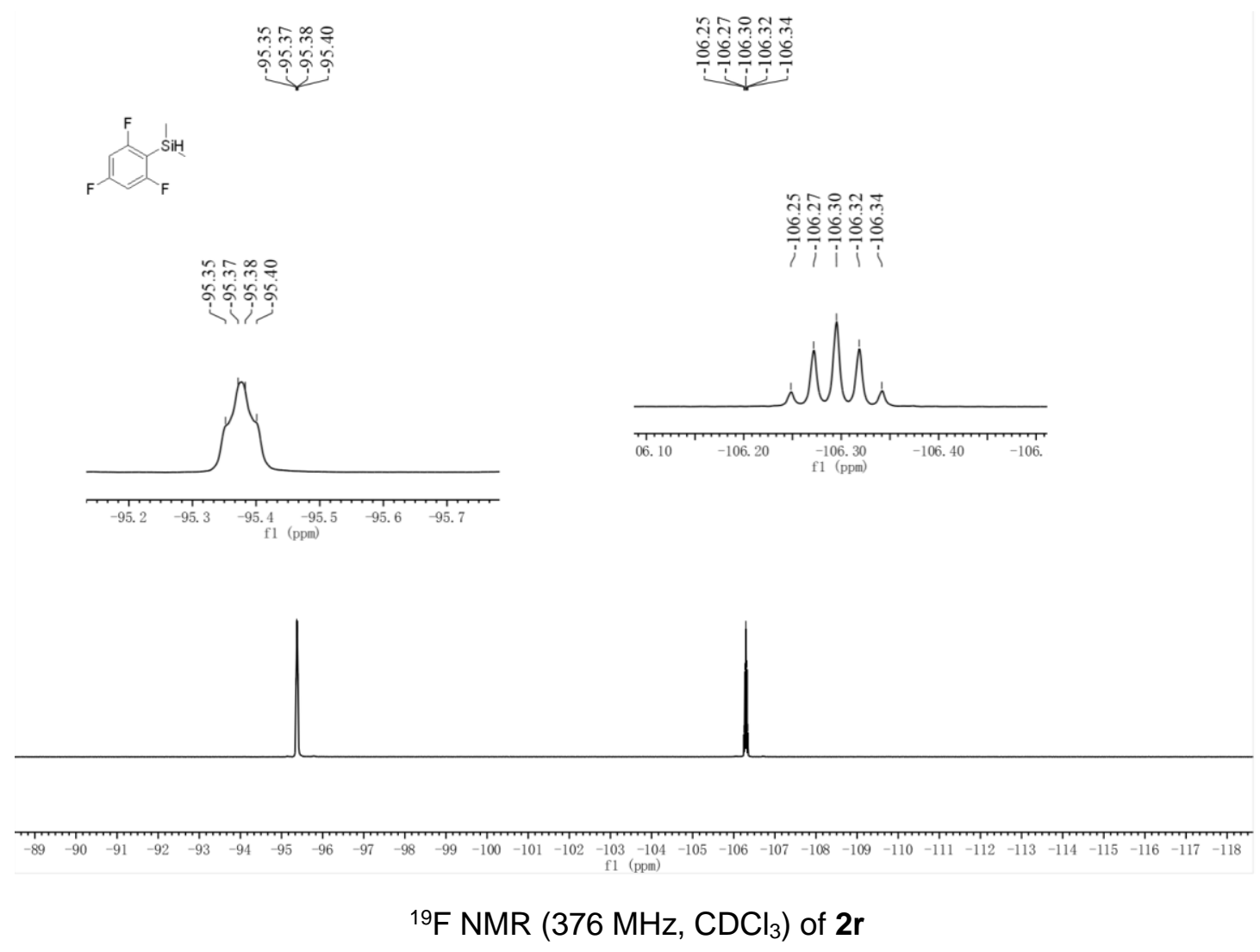


Sit

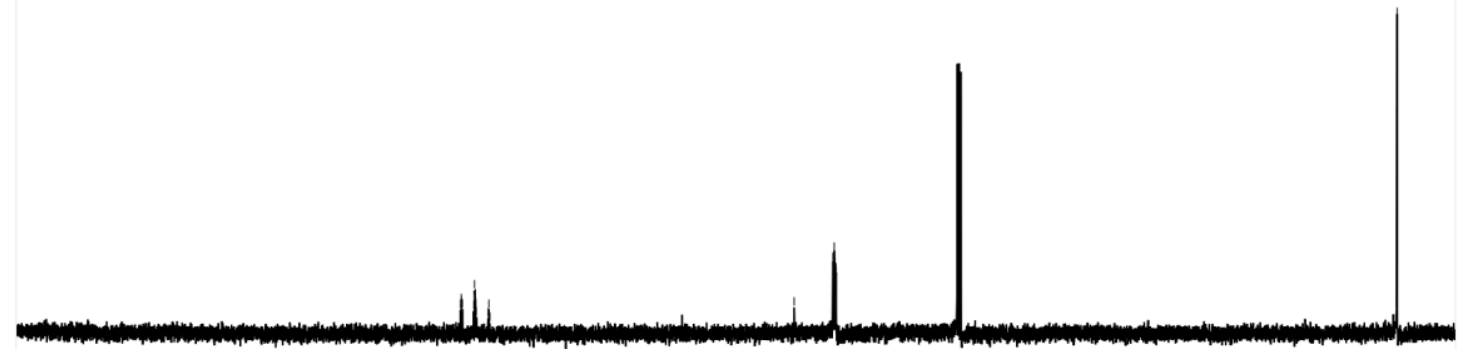

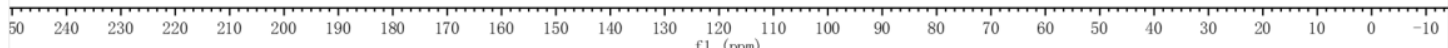

${ }^{13} \mathrm{C}$ NMR $\left(101 \mathrm{MHz} \mathrm{CDCl}_{3}\right)$ of $\mathbf{2 r}$ 

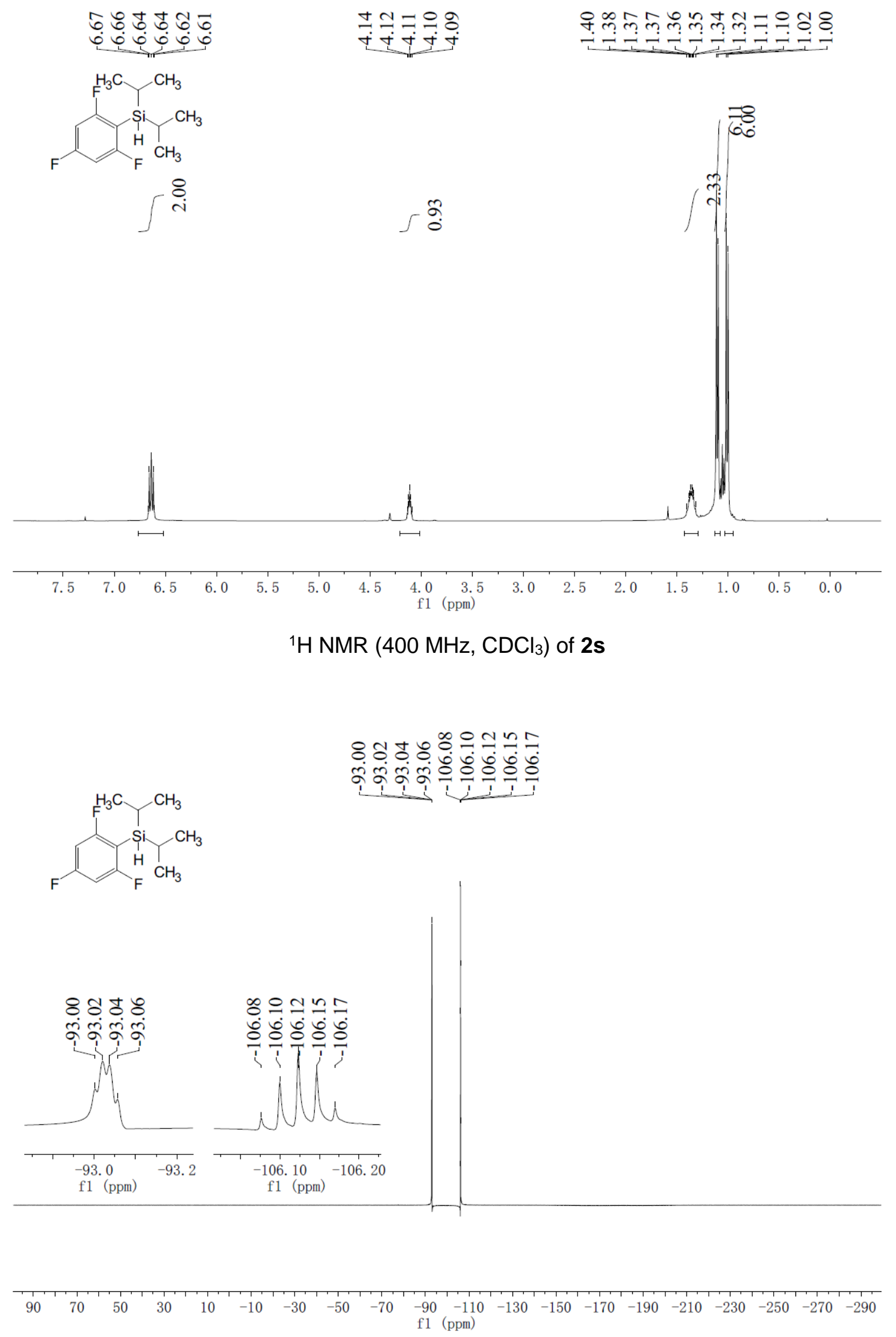

$\left.{ }^{19} \mathrm{~F} \mathrm{NMR} \mathrm{(376} \mathrm{MHz,} \mathrm{CDCl}_{3}\right)$ of 2 s 


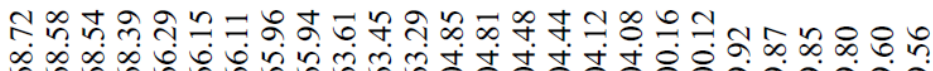

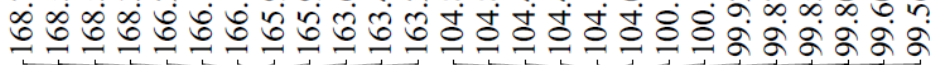

б.

$\infty \infty 00$

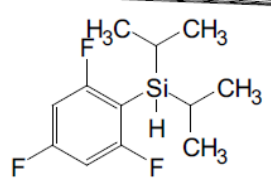

$\begin{array}{llllllllllllllllllllllllllllll}230 & 220 & 210 & 200 & 190 & 180 & 170 & 160 & 150 & 140 & 130 & 120 & 110 & 100 & 90 & 80 & 70 & 60 & 50 & 40 & 30 & 20 & 10 & 0 & -10\end{array}$ f1 (ppm)

${ }^{13} \mathrm{C} \mathrm{NMR}\left(101 \mathrm{MHz}, \mathrm{CDCl}_{3}\right)$ of $\mathbf{2 s}$ 


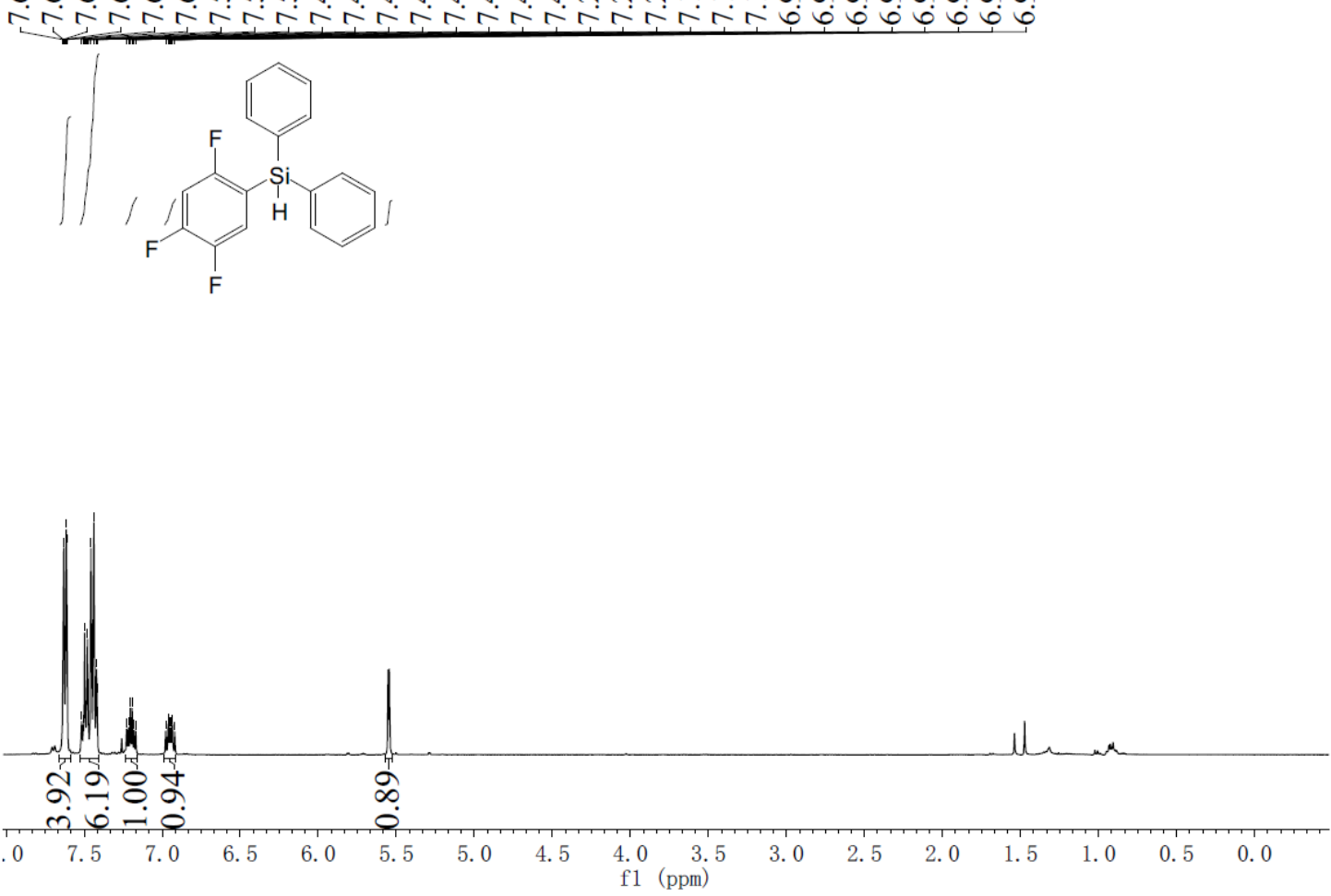

${ }^{1} \mathrm{H}$ NMR $\left(400 \mathrm{MHz}, \mathrm{CDCl}_{3}\right)$ of $\mathbf{2 t}$

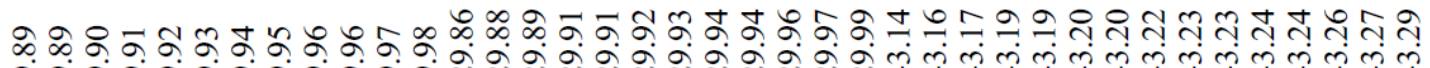

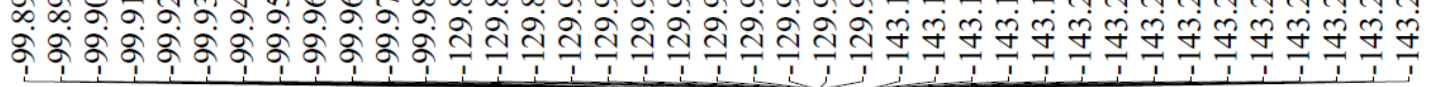<smiles>Fc1cc(F)c([SiH](c2ccccc2)c2ccccc2)cc1F</smiles>

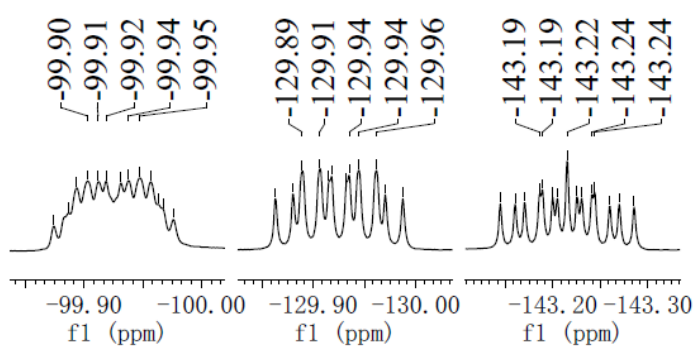

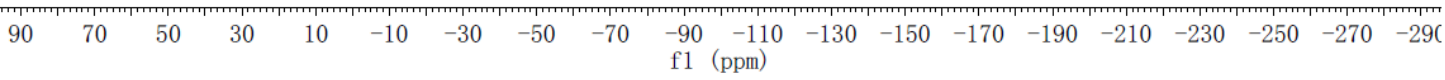
${ }^{19} \mathrm{~F} \mathrm{NMR}\left(376 \mathrm{MHz}, \mathrm{CDCl}_{3}\right)$ of $\mathbf{2 t}$ 


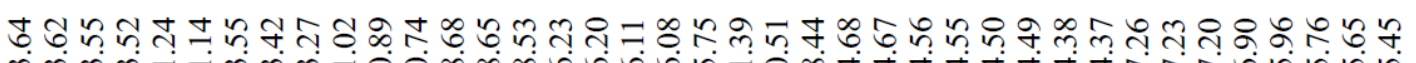

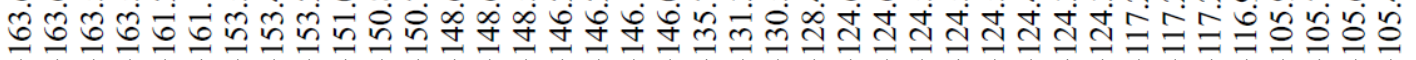

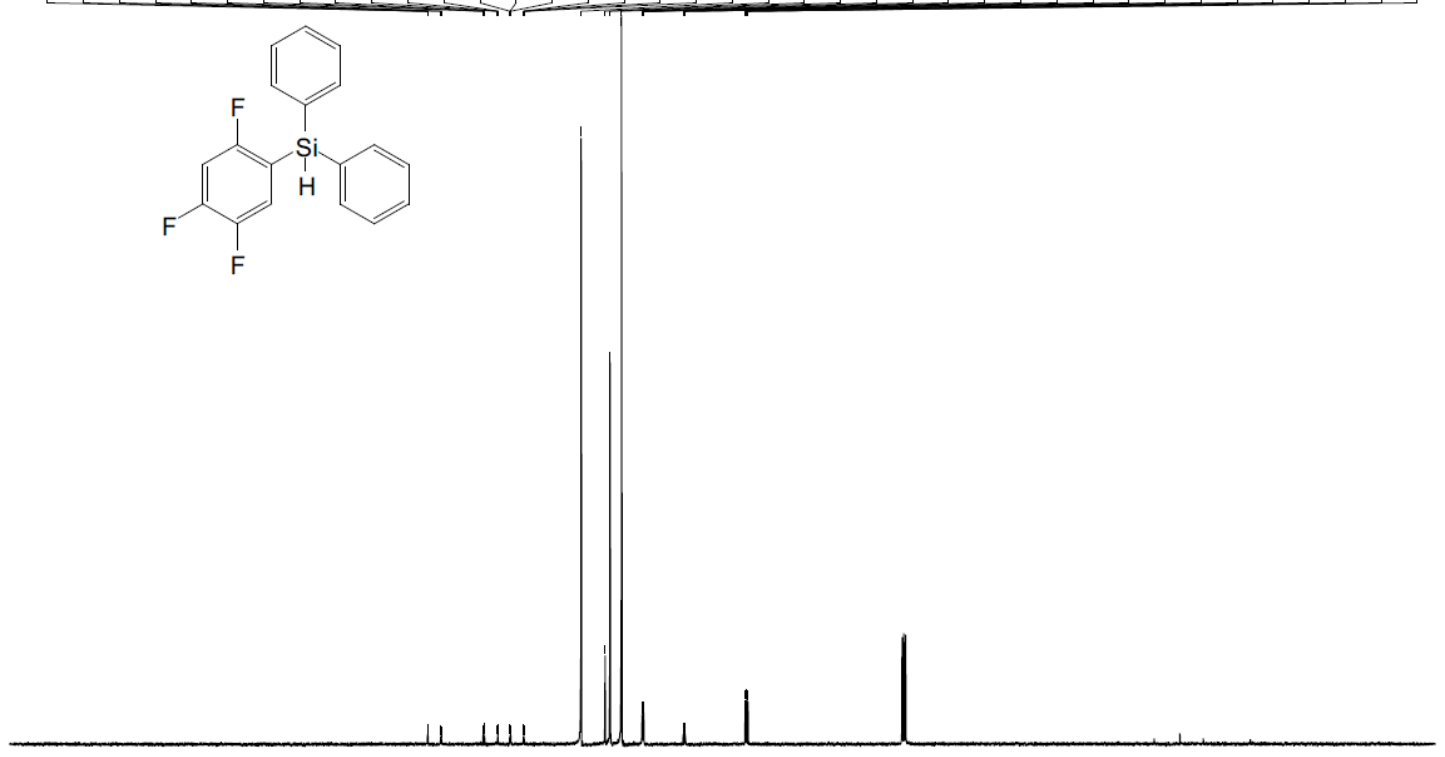

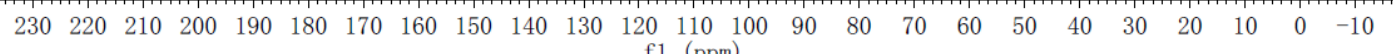
f1 (ppm)

${ }^{13} \mathrm{C}$ NMR $\left(101 \mathrm{MHz}, \mathrm{CDCl}_{3}\right)$ of $\mathbf{2 t}$ 


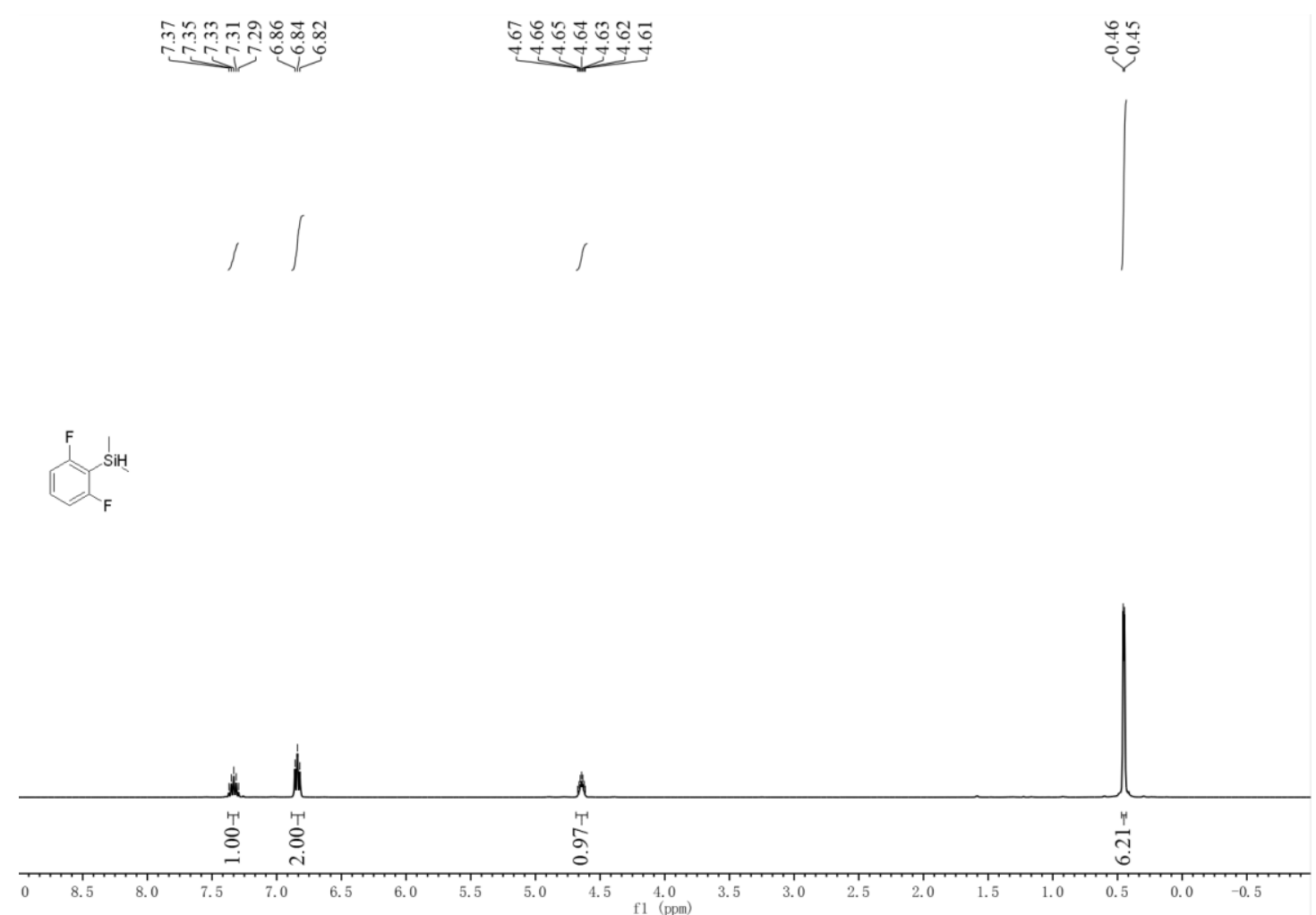

${ }^{1} \mathrm{H}$ NMR $\left(400 \mathrm{MHz}, \mathrm{CDCl}_{3}\right)$ of $\mathbf{2 u}$

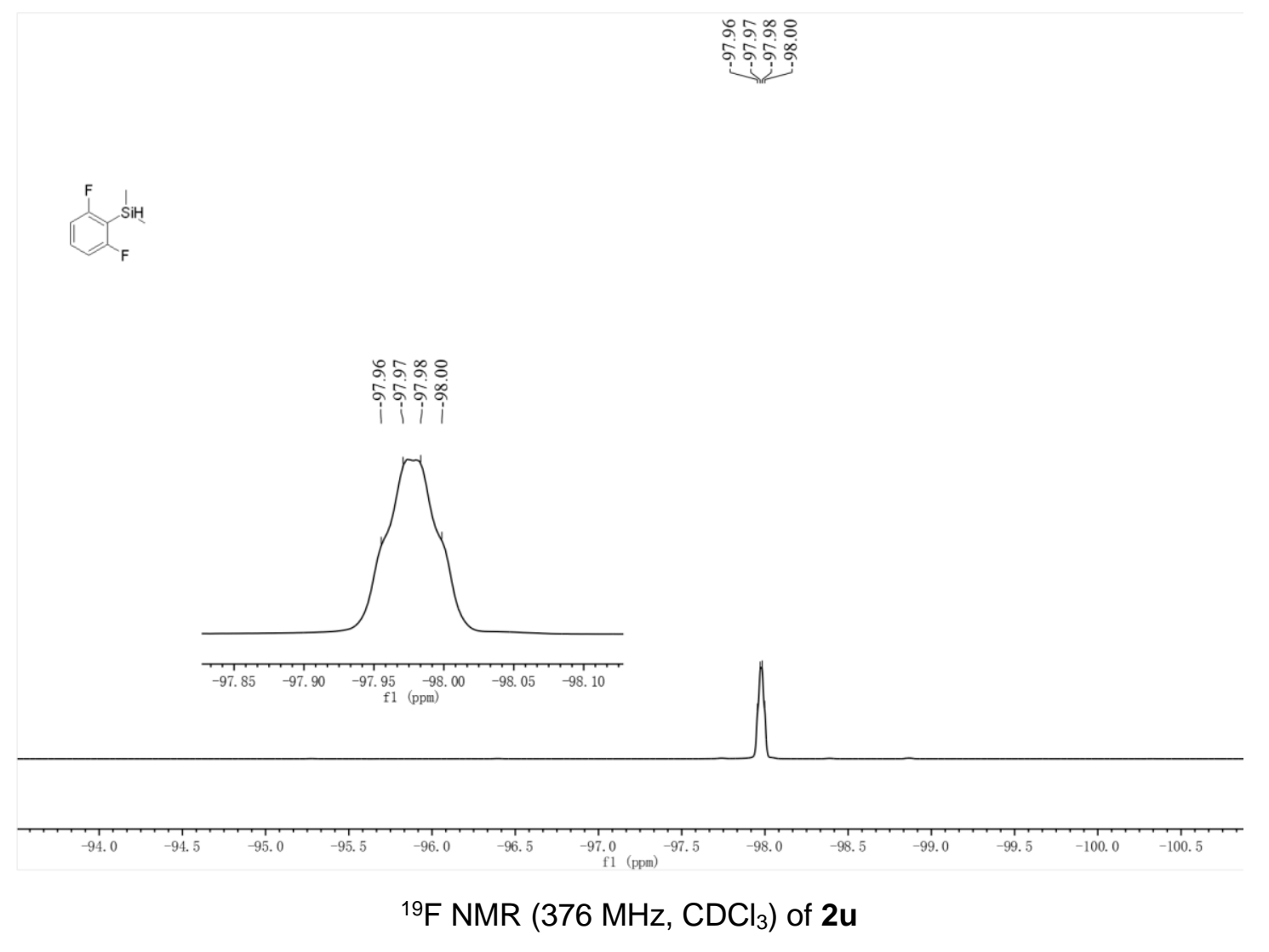




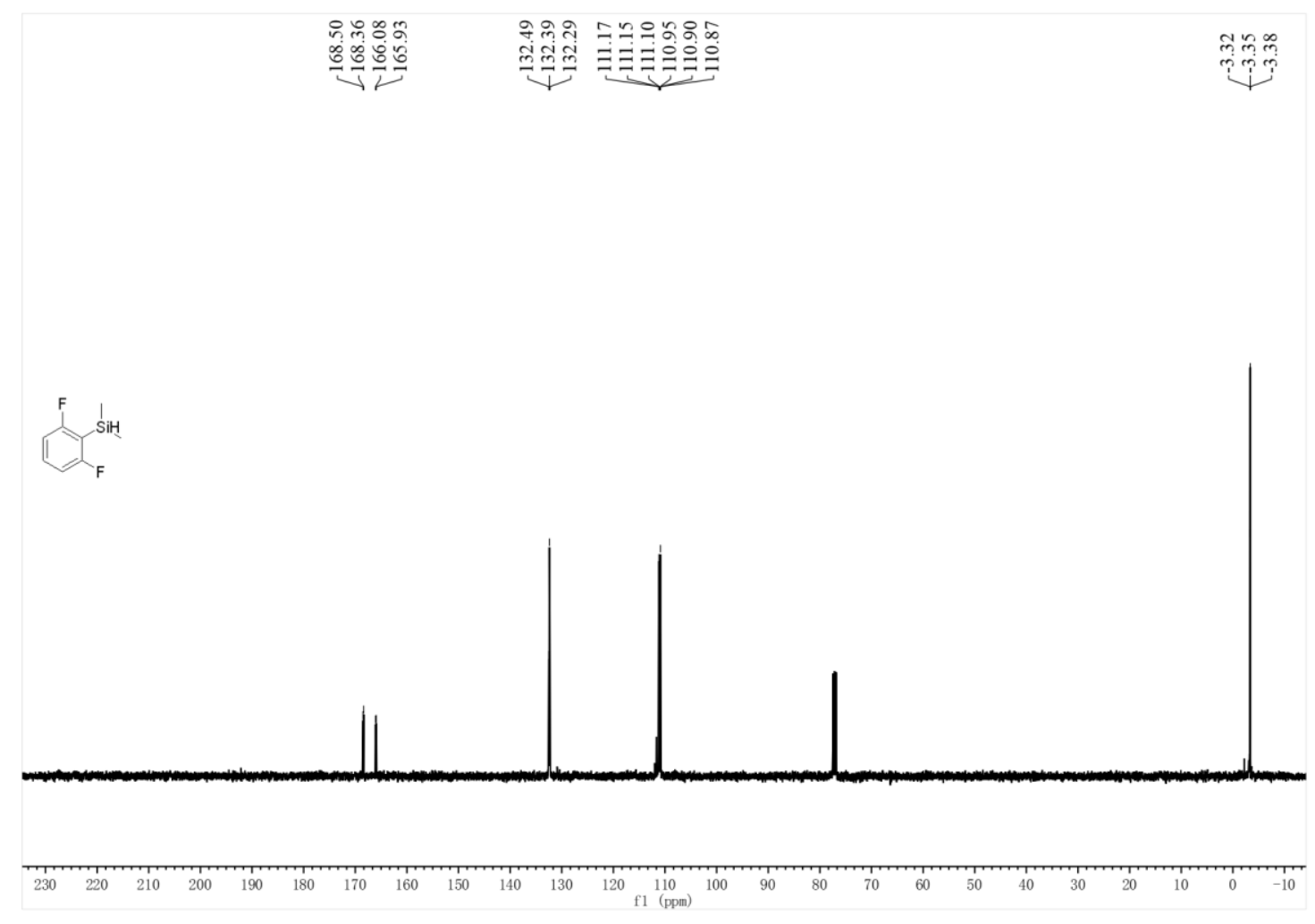

${ }^{13} \mathrm{C} \mathrm{NMR}\left(101 \mathrm{MHz}, \mathrm{CDCl}_{3}\right)$ of $\mathbf{2 u}$ 


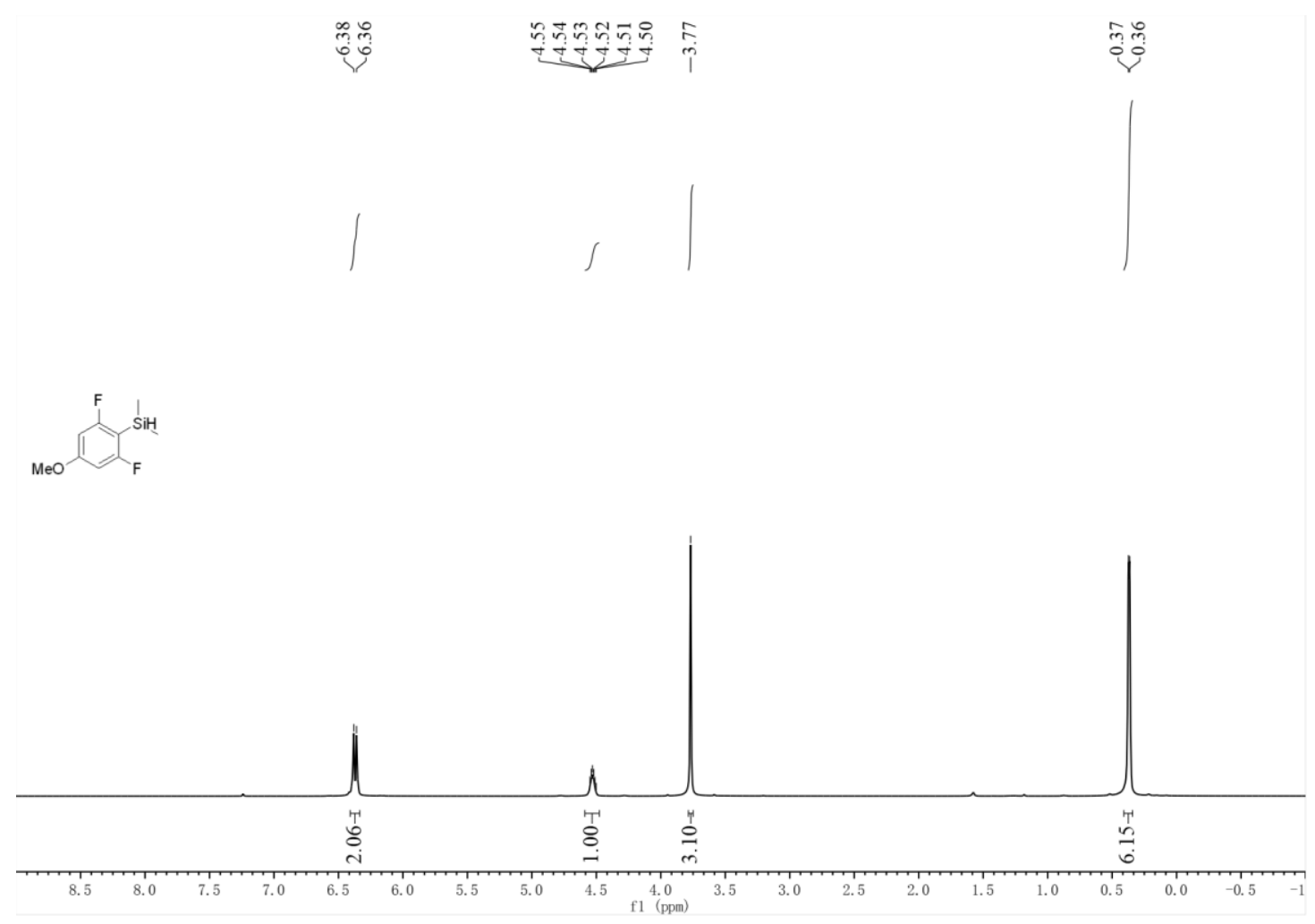

${ }^{1} \mathrm{H}$ NMR $\left(400 \mathrm{MHz}, \mathrm{CDCl}_{3}\right)$ of $\mathbf{2} \mathbf{v}$

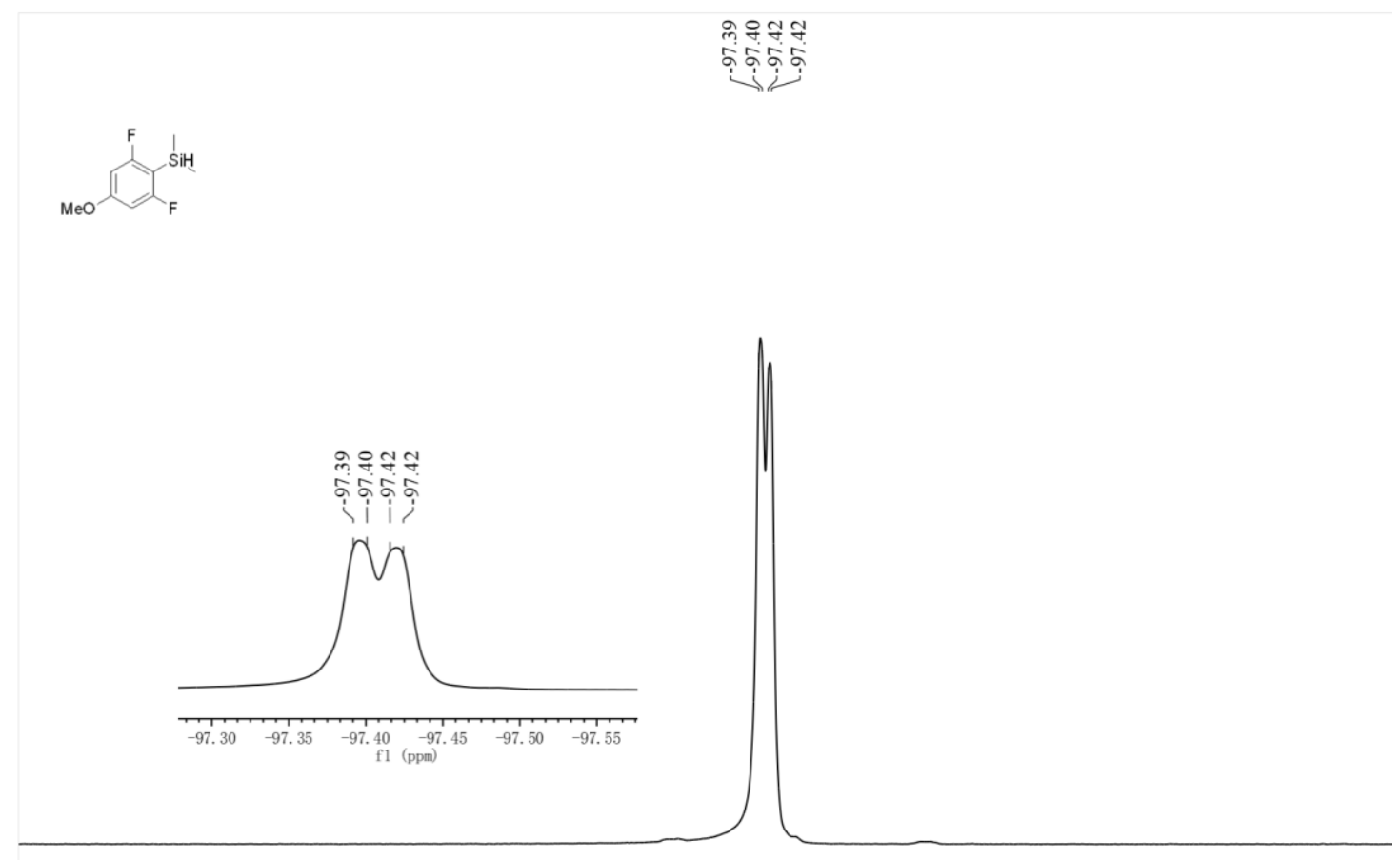

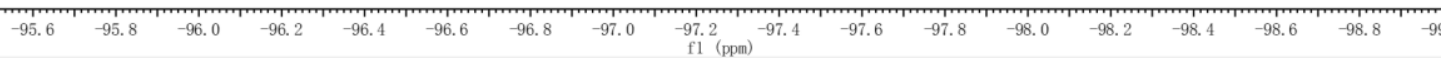

${ }^{19} \mathrm{~F} \mathrm{NMR}\left(376 \mathrm{MHz}, \mathrm{CDCl}_{3}\right)$ of $\mathbf{2 v}$ 

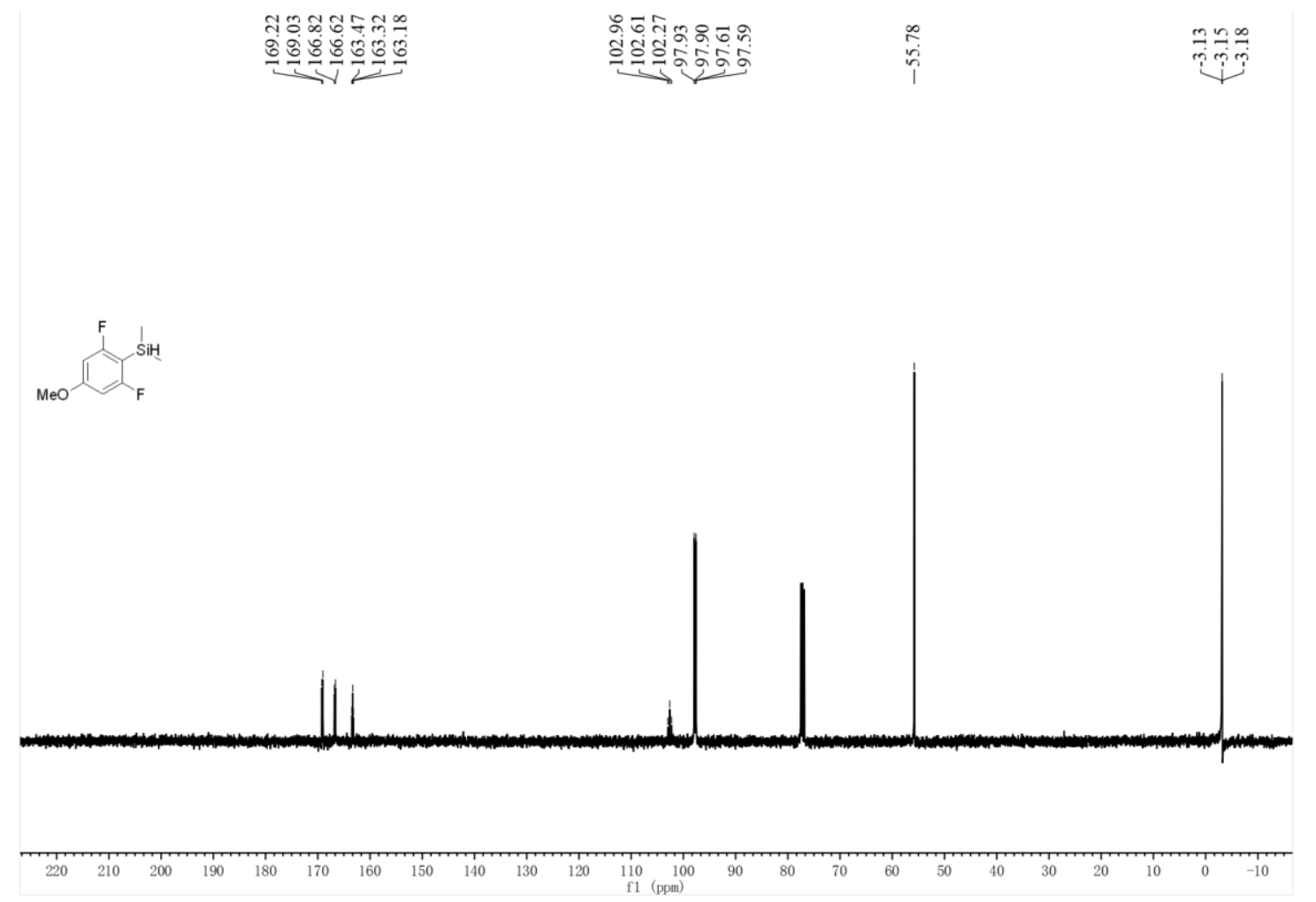

$\left.{ }^{13} \mathrm{C} \mathrm{NMR} \mathrm{(101} \mathrm{MHz,} \mathrm{CDCl}_{3}\right)$ of $\mathbf{2 v}$ 


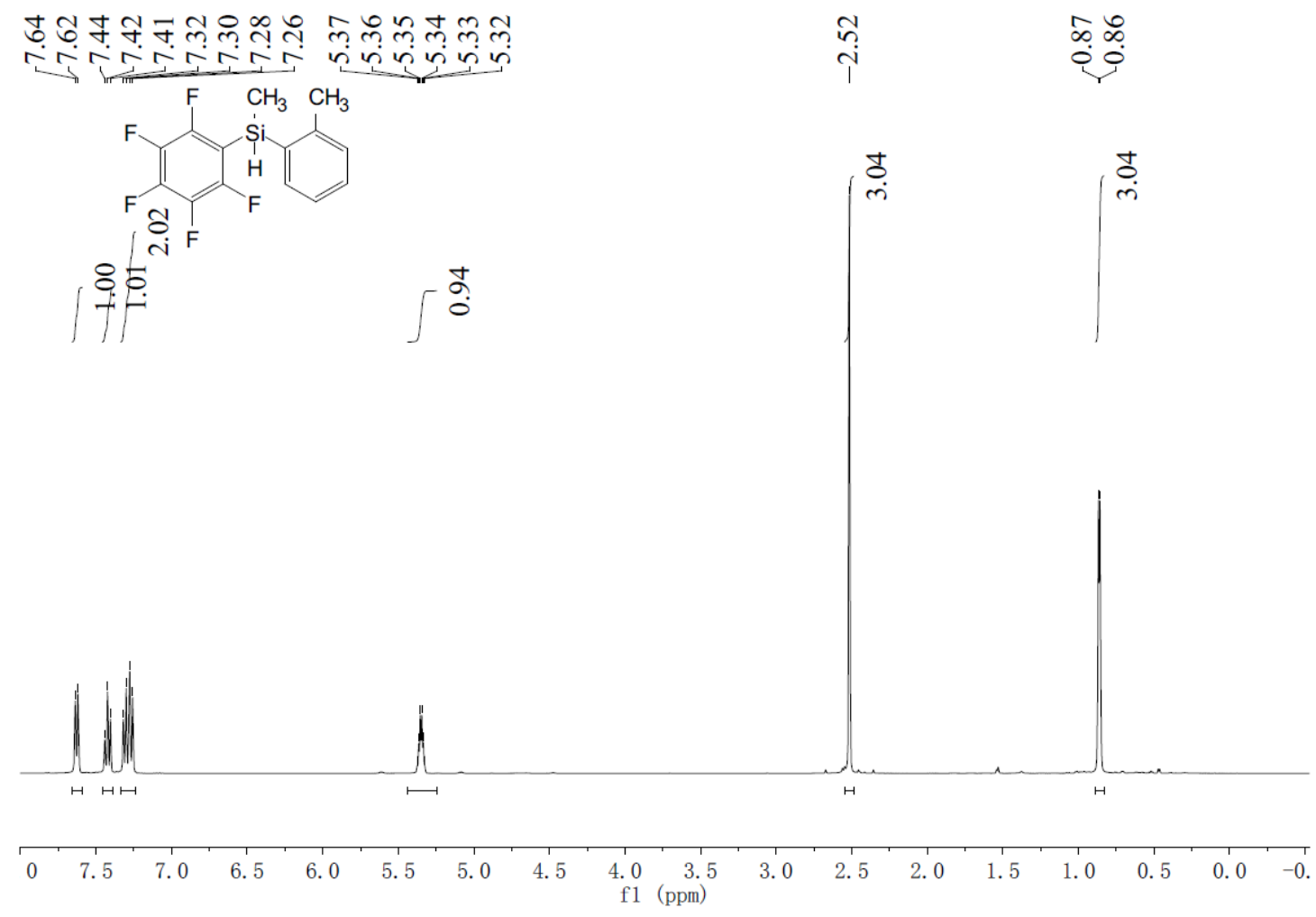

${ }^{1} \mathrm{H}$ NMR $\left(400 \mathrm{MHz}, \mathrm{CDCl}_{3}\right)$ of $5 \mathbf{a}$<smiles>Cc1ccccc1[Si](C)c1c(F)c(F)c(F)c(F)c1F</smiles>

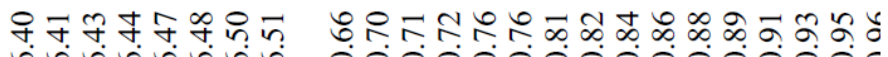

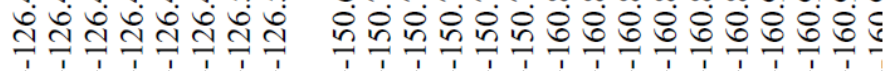

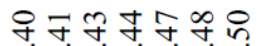

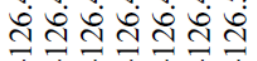
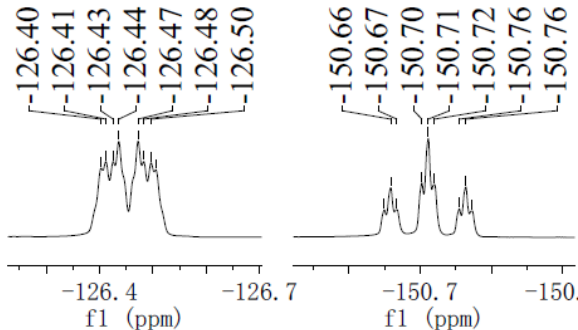

\section{은}

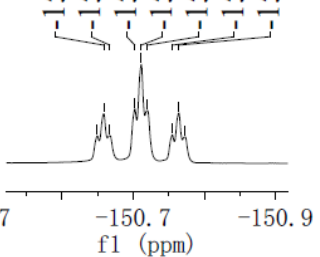

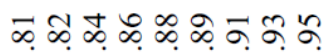

888888880
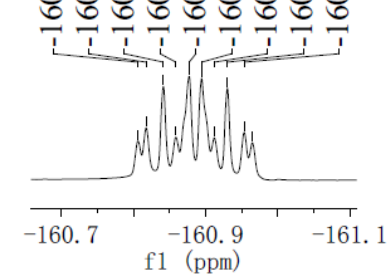

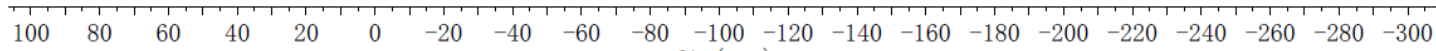
f1 (ppm)

${ }^{19} \mathrm{~F} \mathrm{NMR}\left(376 \mathrm{MHz}, \mathrm{CDCl}_{3}\right)$ of $\mathbf{5 a}$ 


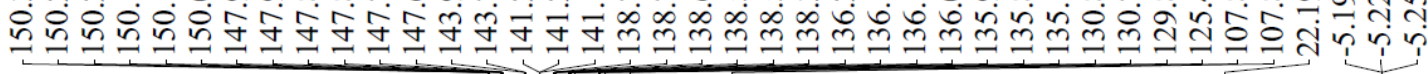<smiles>Cc1ccccc1C(C)c1c(F)c(F)c(F)c(F)c1F</smiles>

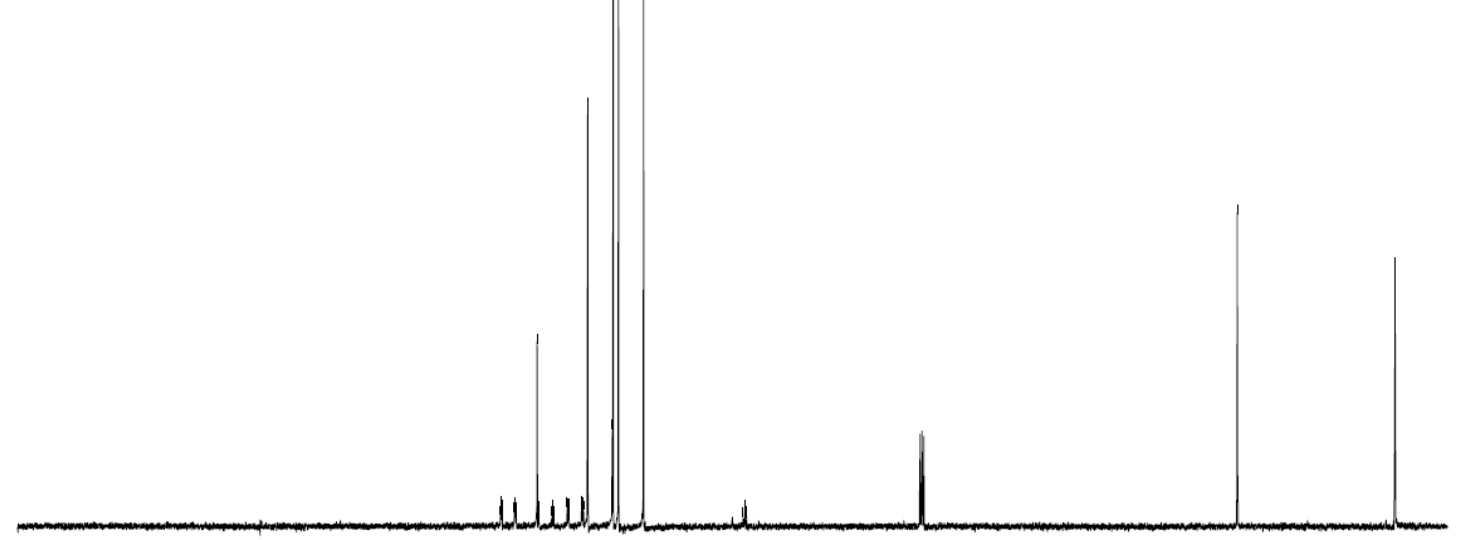

$\begin{array}{lllllllllllllllllllllllllllll}230 & 220 & 210 & 200 & 190 & 180 & 170 & 160 & 150 & 140 & 130 & 120 & 110 & 100 & 90 & 80 & 70 & 60 & 50 & 40 & 30 & 20 & 10 & 0 & -10\end{array}$

${ }^{13} \mathrm{C} \mathrm{NMR}\left(101 \mathrm{MHz}, \mathrm{CDCl}_{3}\right)$ of $\mathbf{5 a}$ 


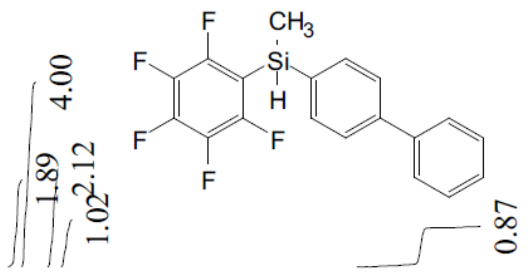
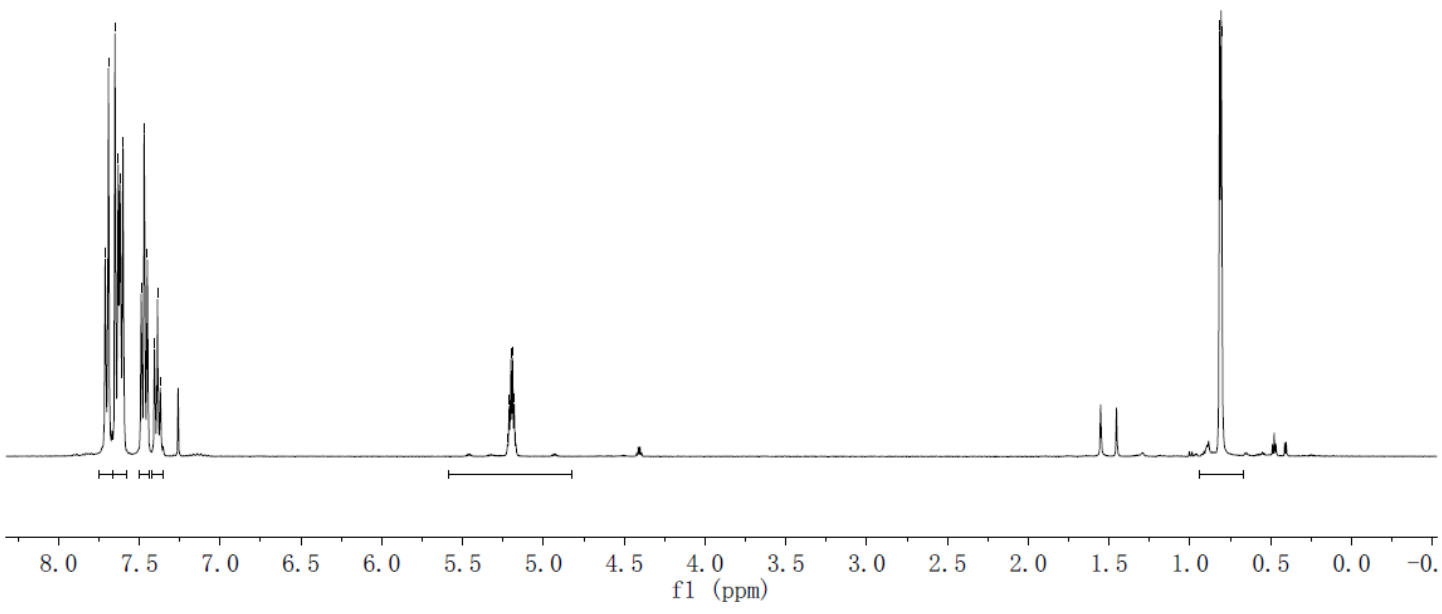

${ }^{1} \mathrm{H} \mathrm{NMR}\left(400 \mathrm{MHz}, \mathrm{CDCl}_{3}\right)$ of $\mathbf{5 b}$
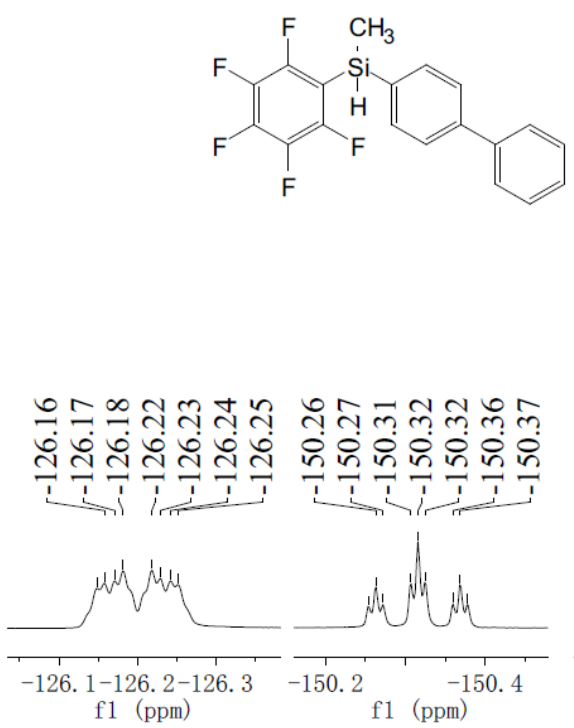

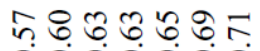
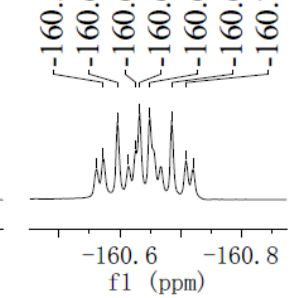

$\begin{array}{lllllll}140 & 120 & 100 & 80 & 60 & 40 & 20\end{array}$

${ }^{19} \mathrm{~F} \mathrm{NMR}\left(376 \mathrm{MHz}, \mathrm{CDCl}_{3}\right)$ of $\mathbf{5 b}$ 
ஏ

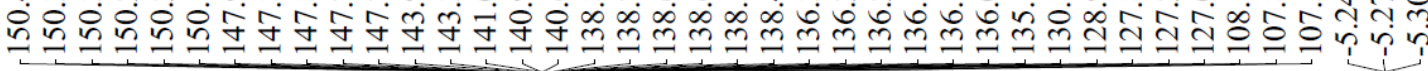<smiles>CC(C)CCSc1c(F)c(F)c(F)c(F)c1Sc1ccc(-c2ccccc2)cc1</smiles>

$\begin{array}{llllllllllllll}230 & 220 & 210 & 200 & 190 & 180 & 170 & 160 & 150 & 140 & 130 & 120 & 110 & 100\end{array}$ f1 (ppm)

${ }^{13} \mathrm{C} \mathrm{NMR}\left(101 \mathrm{MHz}, \mathrm{CDCl}_{3}\right)$ of $\mathbf{5 b}$ 


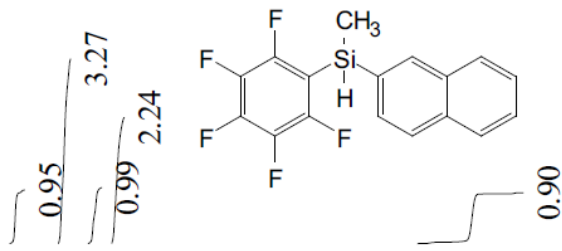
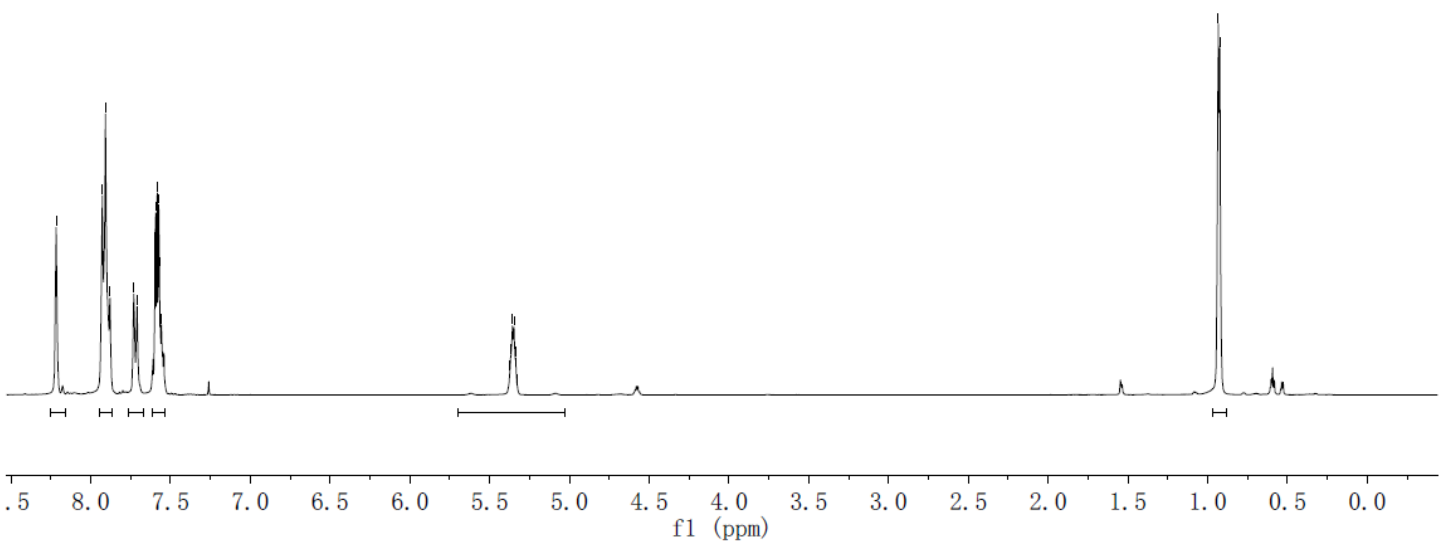

${ }^{1} \mathrm{H}$ NMR $\left(400 \mathrm{MHz}, \mathrm{CDCl}_{3}\right)$ of $\mathbf{5 c}$

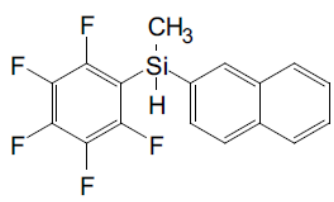

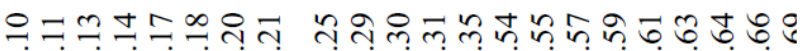
원ำㄴำ

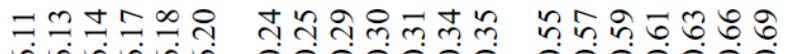

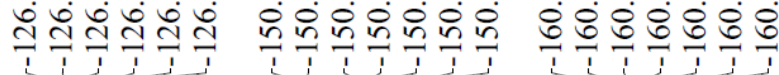

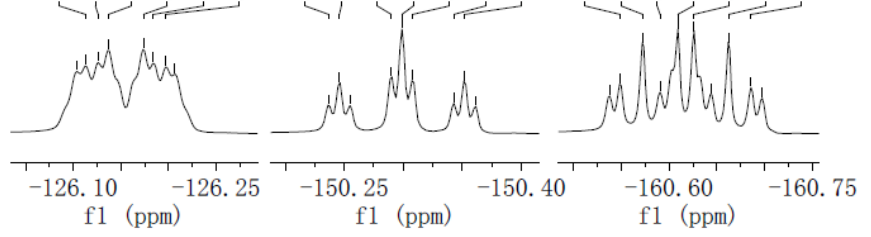

$\begin{array}{lllllllllllllllllllllllllllll}140 & 120 & 100 & 80 & 60 & 40 & 20 & 0 & -20 & -40 & -60 & -80 & -100 & -120 & -140 & -160 & -180 & -200 & -220 & -240 & -260 & -280\end{array}$ ${ }^{19} \mathrm{~F} \mathrm{NMR}\left(376 \mathrm{MHz}^{\mathrm{CDCl}} \mathrm{CD}_{3}\right)$ of $5 \mathrm{c}$ 


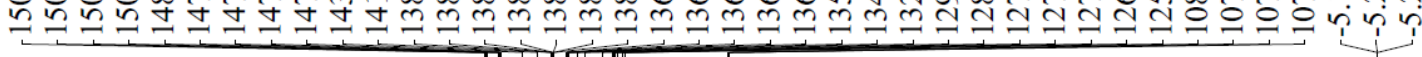<smiles>C[Si](c1ccc2ccccc2c1)c1c(F)c(F)c(F)c(F)c1F</smiles>

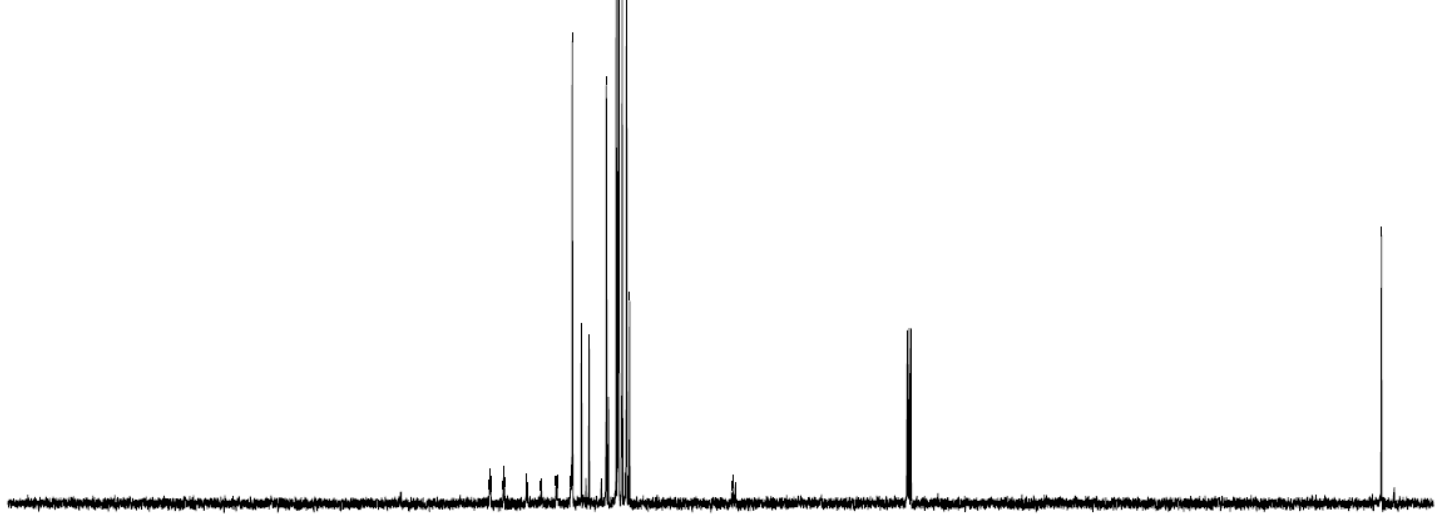

$\begin{array}{lllllllllllllllllllllllll}230 & 220 & 210 & 200 & 190 & 180 & 170 & 160 & 150 & 140 & 130 & 120 & 110 & 100 & 90 & 80 & 70 & 60 & 50 & 40 & 30 & 20 & 10 & 0 & -10\end{array}$

f1 (ppm)

${ }^{13} \mathrm{C} \mathrm{NMR}\left(101 \mathrm{MHz}, \mathrm{CDCl}_{3}\right)$ of $5 \mathrm{c}$ 


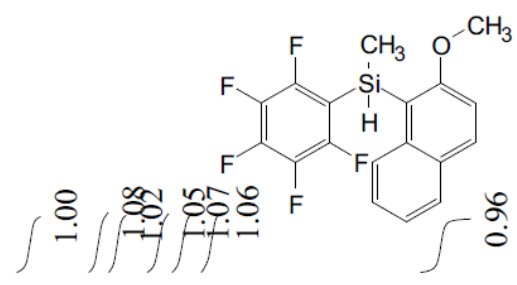

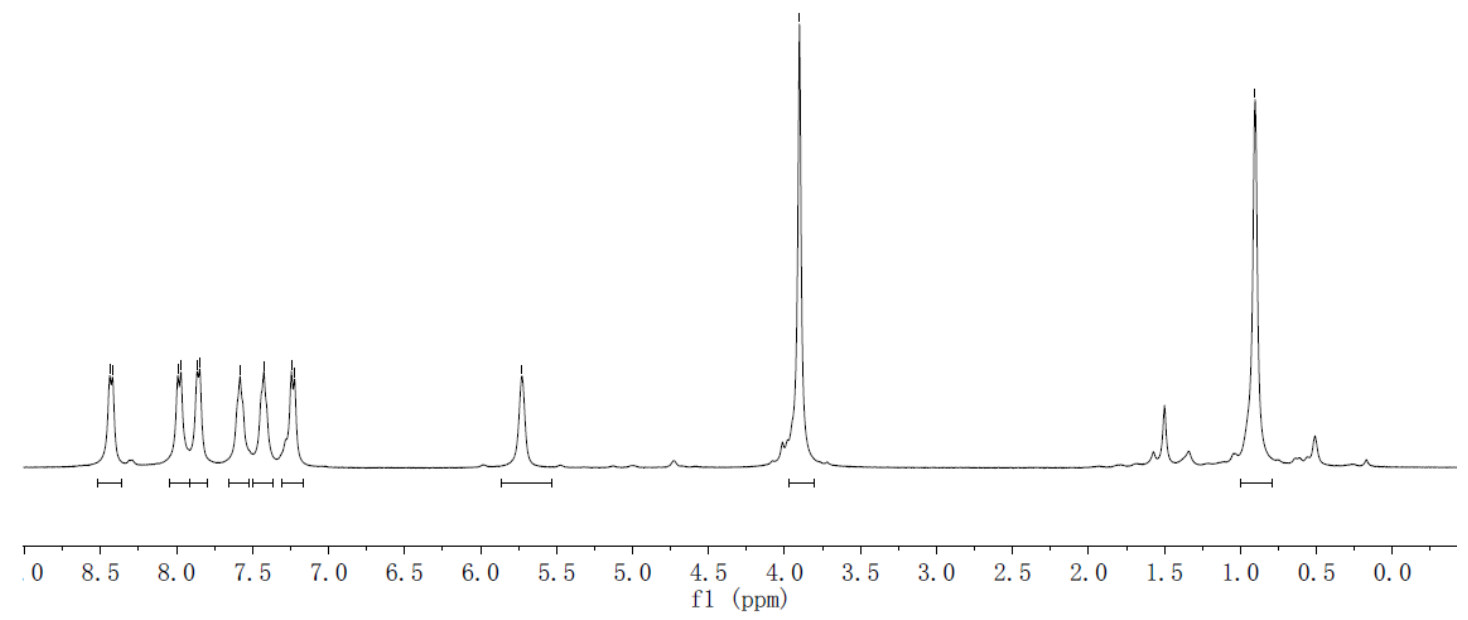

${ }^{1} \mathrm{H}$ NMR $\left(400 \mathrm{MHz}, \mathrm{CDCl}_{3}\right)$ of $\mathbf{5 d}$<smiles>COc1ccc2cccc3c2c1[Si](C)c1c(F)c(F)c(F)c(F)c1-3</smiles>

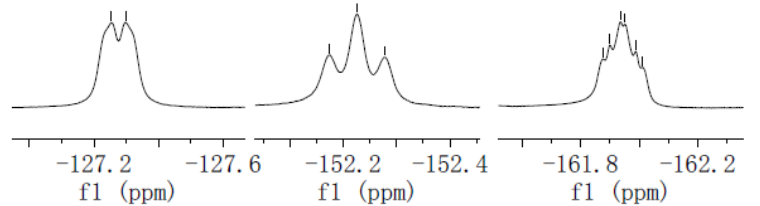


<smiles>COc1ccc2cccc3c2c1[Si](C)(C)c1c(F)c(F)c(F)c(F)c1-3</smiles>

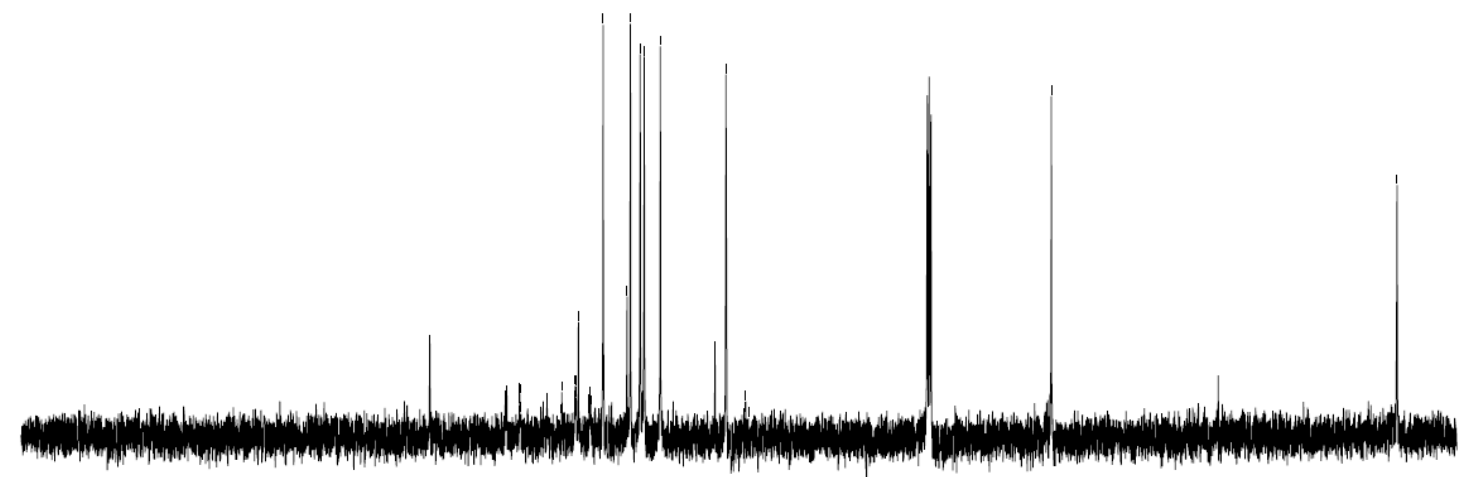

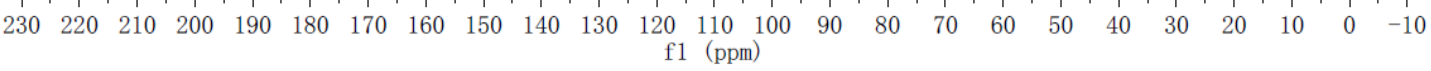
${ }^{13} \mathrm{C} \mathrm{NMR}\left(101 \mathrm{MHz}, \mathrm{CDCl}_{3}\right)$ of $\mathbf{5 d}$ 

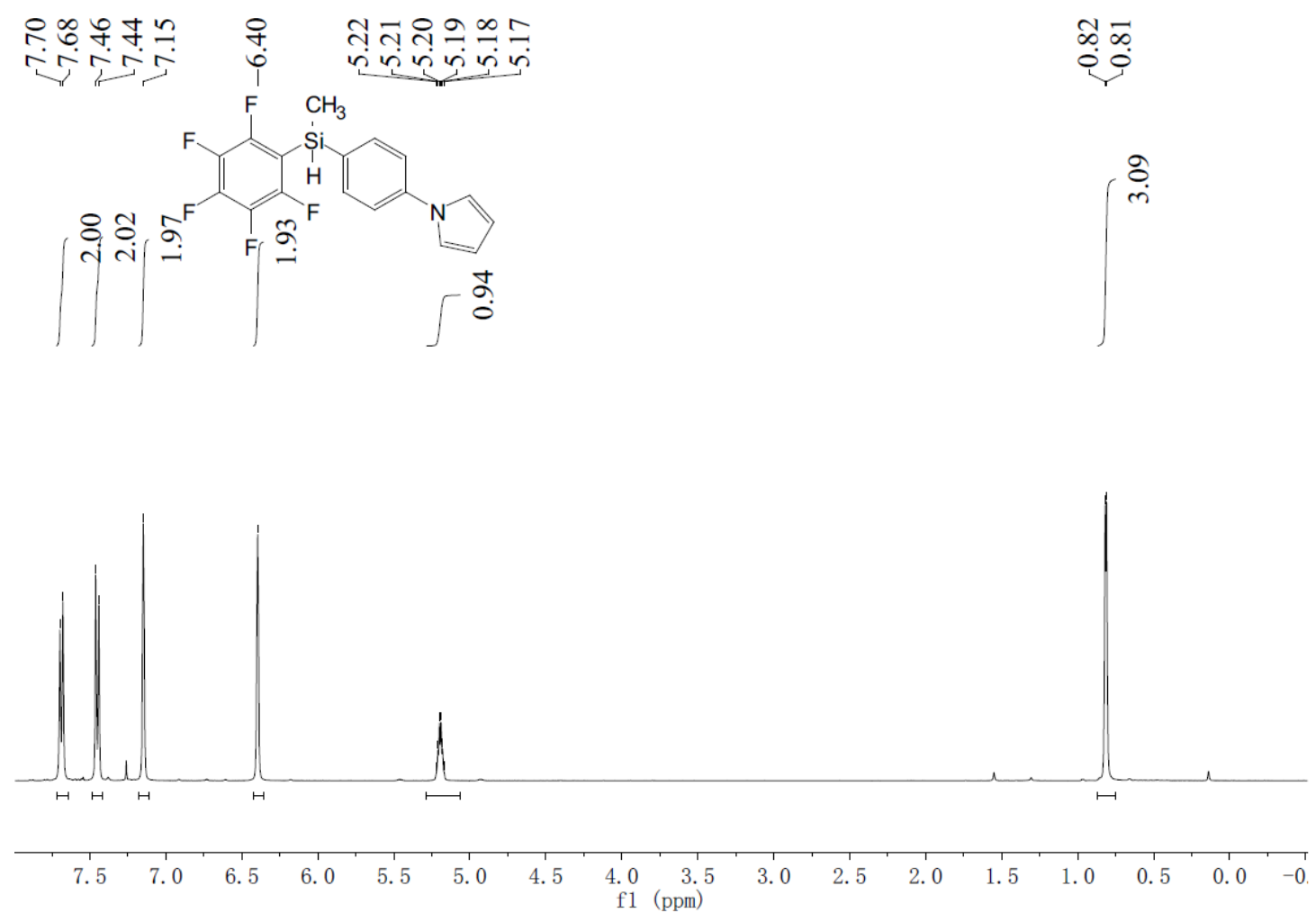

${ }^{1} \mathrm{H}$ NMR $\left(400 \mathrm{MHz}, \mathrm{CDCl}_{3}\right)$ of $5 e$

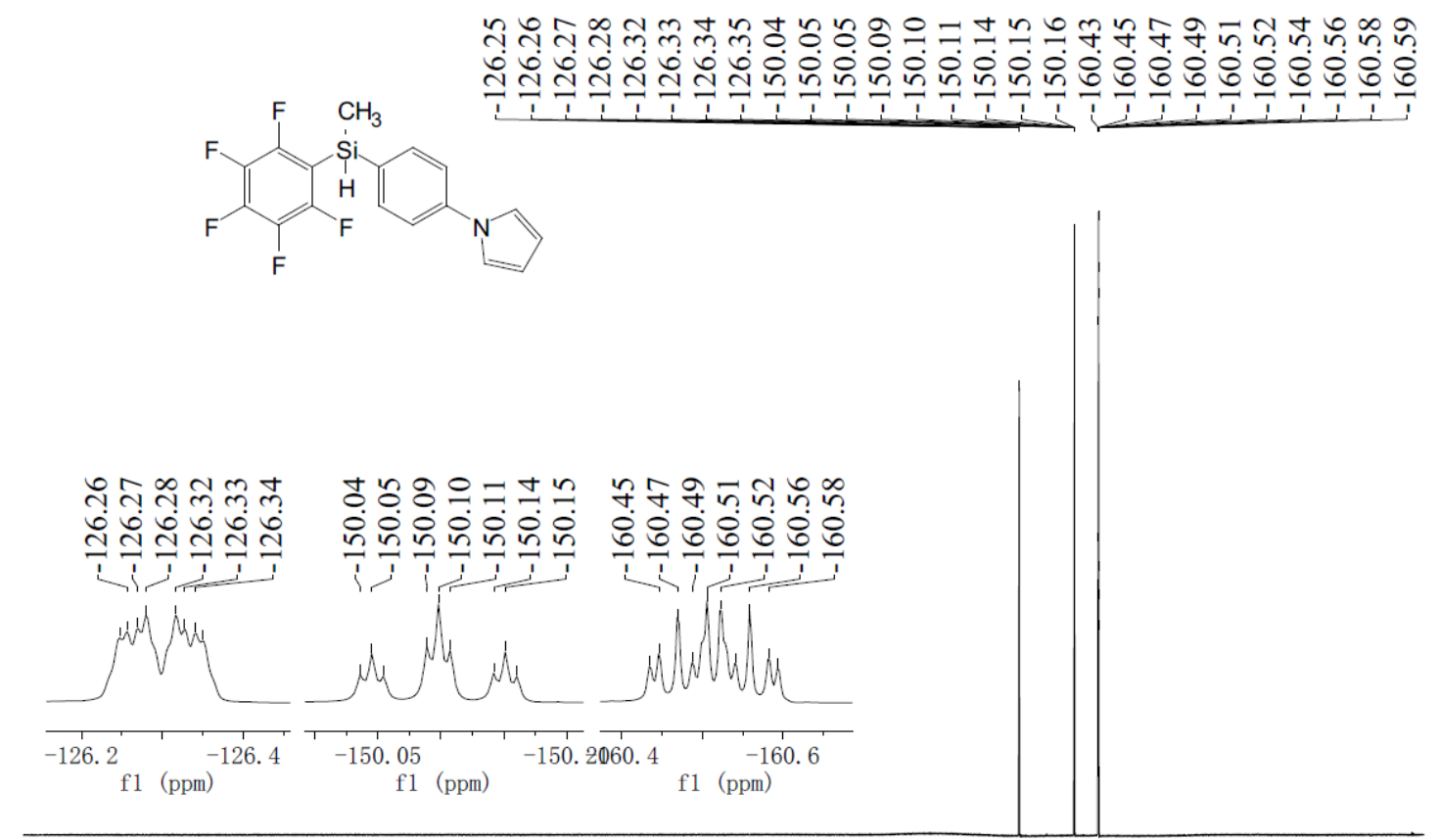

$\stackrel{7}{00}$

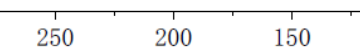

100

50

f1 (ppm)

${ }^{19} \mathrm{~F} \mathrm{NMR}\left(376 \mathrm{MHz}, \mathrm{CDCl}_{3}\right)$ of $5 \mathrm{e}$ 


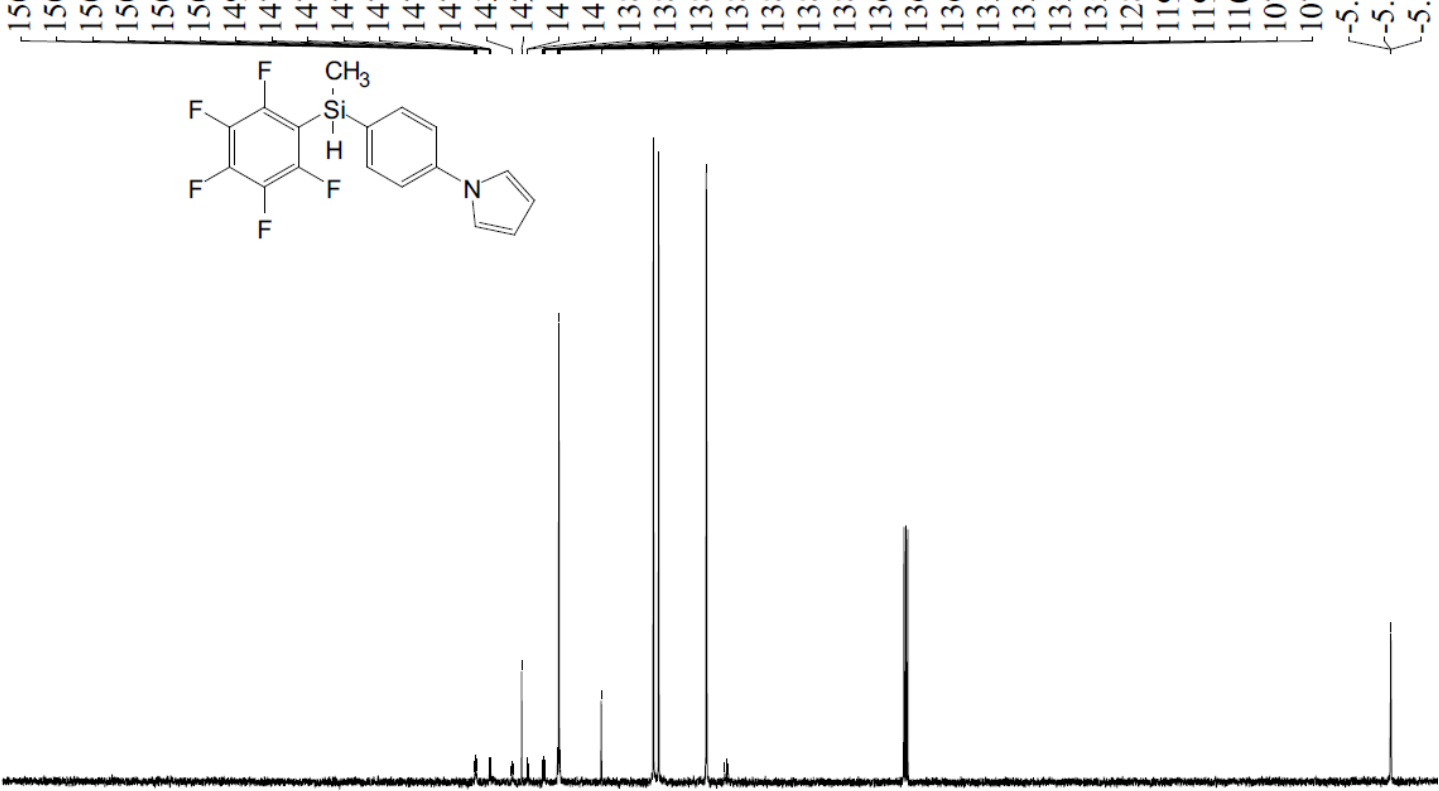

$\begin{array}{llllllllllllllllllllllllll}30 & 220 & 210 & 200 & 190 & 180 & 170 & 160 & 150 & 140 & 130 & 120 & 110 & 100 & 90 & 80 & 70 & 60 & 50 & 40 & 30 & 20 & 10 & 0 & -10\end{array}$

${ }^{13} \mathrm{C} \mathrm{NMR}\left(101 \mathrm{MHz}, \mathrm{CDCl}_{3}\right)$ of $\mathbf{5 e}$ 


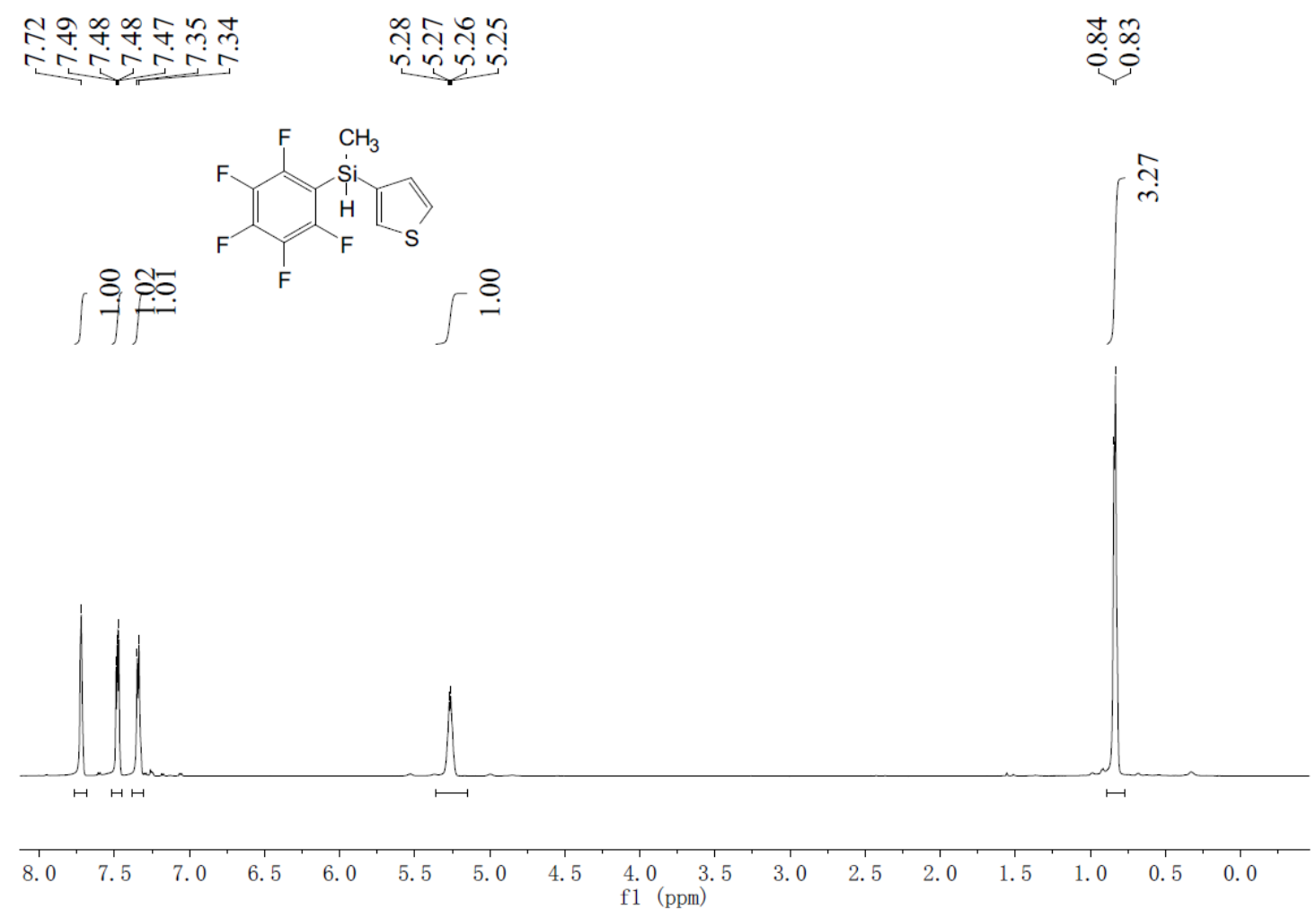

${ }^{1} \mathrm{H}$ NMR $\left(400 \mathrm{MHz}, \mathrm{CDCl}_{3}\right)$ of $\mathbf{5 f}$

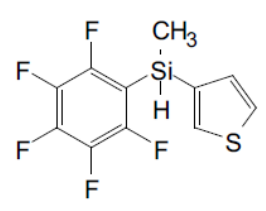

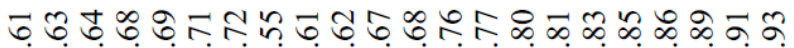

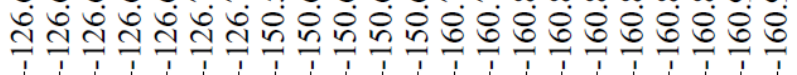

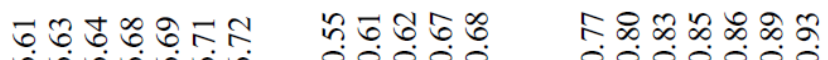

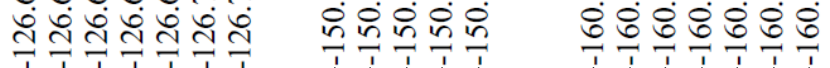

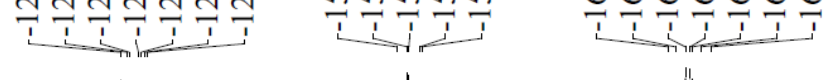

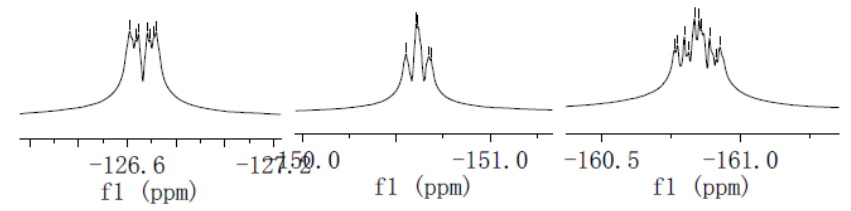

\begin{tabular}{|c|c|c|c|c|c|c|c|c|c|c|}
\hline 250 & 200 & 150 & 100 & 50 & $\mathrm{f}{ }^{-50}(\mathrm{ppm})^{-50}$ & -100 & -150 & -200 & -250 & -300 \\
\hline
\end{tabular}

${ }^{19} \mathrm{~F} \mathrm{NMR}\left(376 \mathrm{MHz}, \mathrm{CDCl}_{3}\right)$ of $\mathbf{5 f}$ 


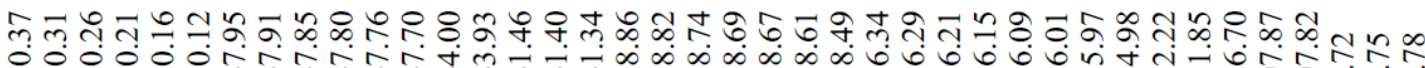

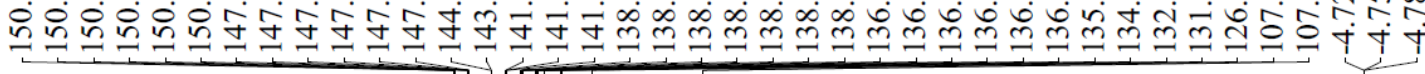<smiles>C[Si](c1ccsc1)c1c(F)c(F)c(F)c(F)c1F</smiles>

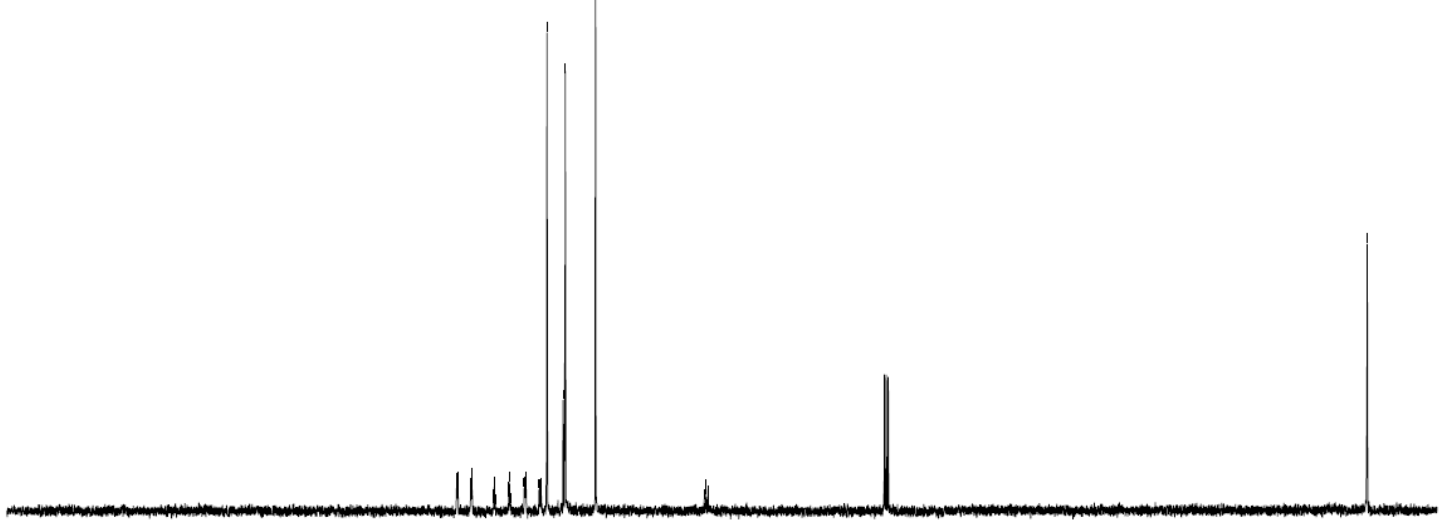

$\begin{array}{llllllllllllllllllllllll}220 & 210 & 200 & 190 & 180 & 170 & 160 & 150 & 140 & 130 & 120 & 110 & 100 & 90 & 80 & 70 & 60 & 50 & 40 & 30 & 20 & 10 & 0 & -10\end{array}$ f1 (ppm)

${ }^{13} \mathrm{C}$ NMR $\left(101 \mathrm{MHz}, \mathrm{CDCl}_{3}\right)$ of $\mathbf{5 f}$ 

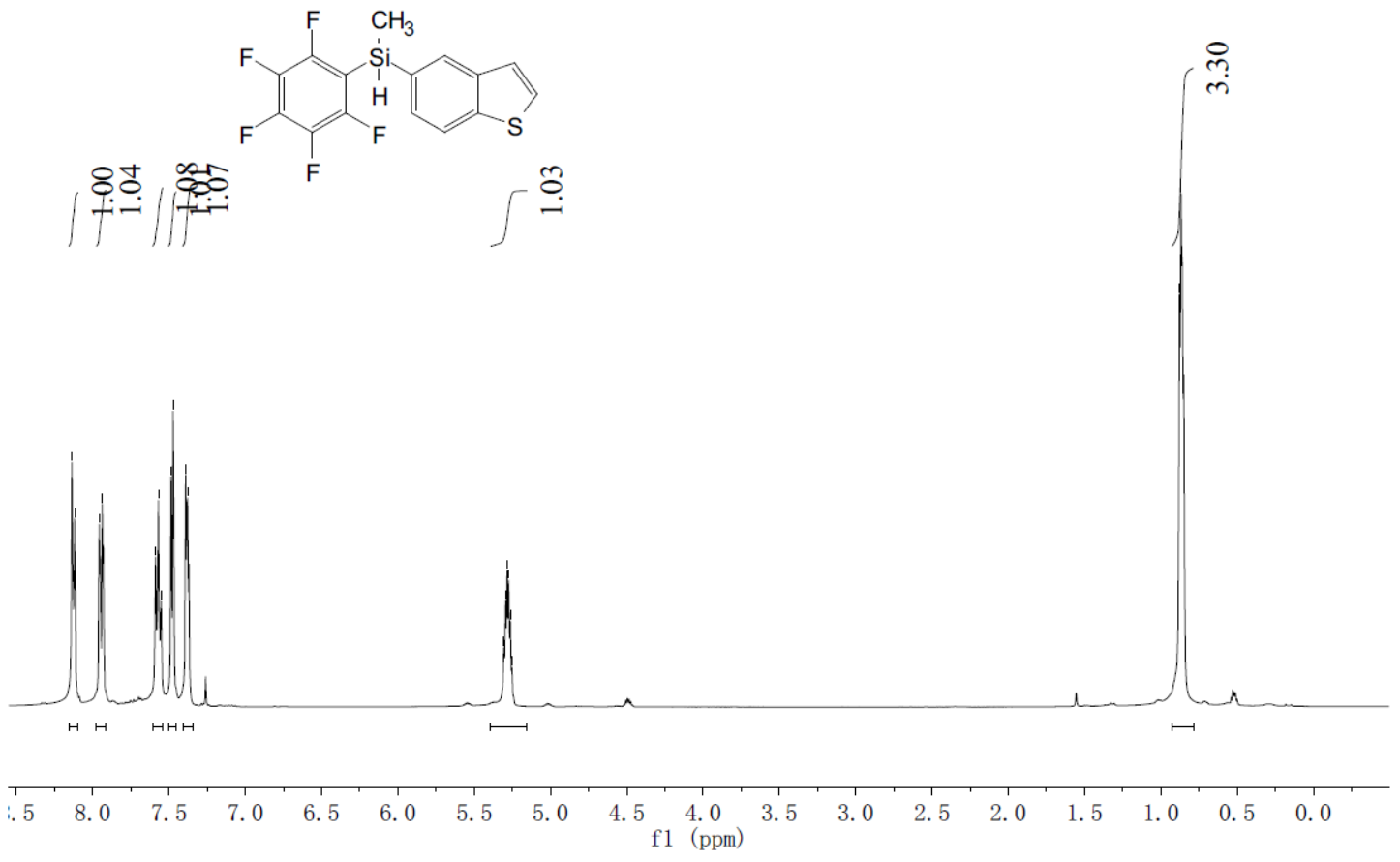

${ }^{1} \mathrm{H}$ NMR $\left(400 \mathrm{MHz}, \mathrm{CDCl}_{3}\right)$ of $\mathbf{5 g}$

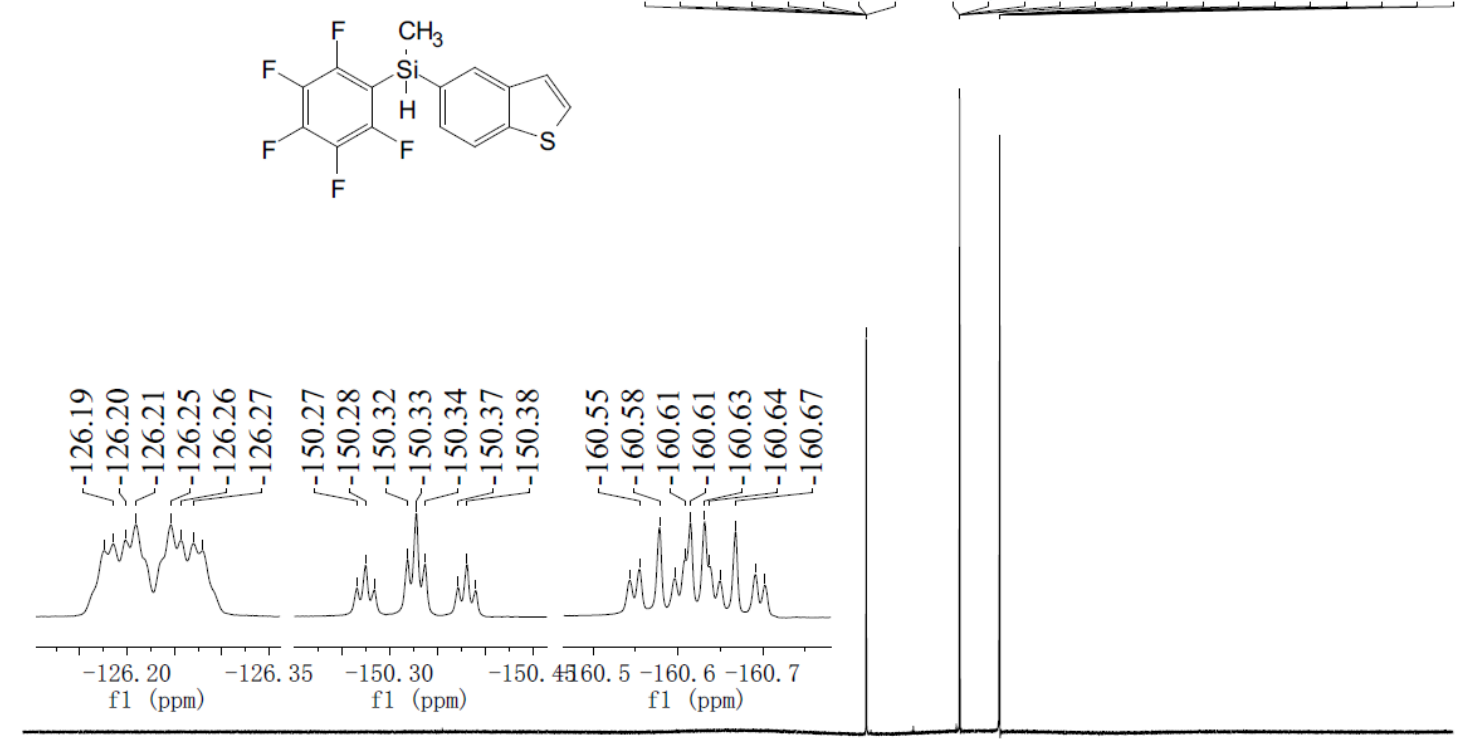

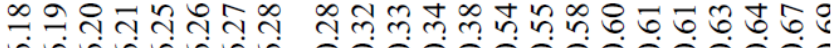

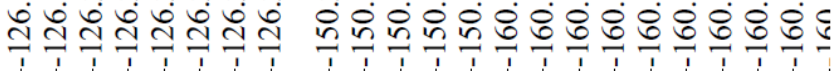

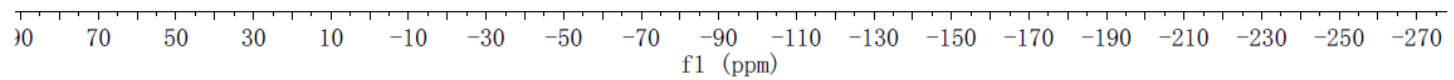
${ }^{19} \mathrm{~F} \mathrm{NMR}\left(376 \mathrm{MHz}, \mathrm{CDCl}_{3}\right)$ of $\mathbf{5 g}$ 


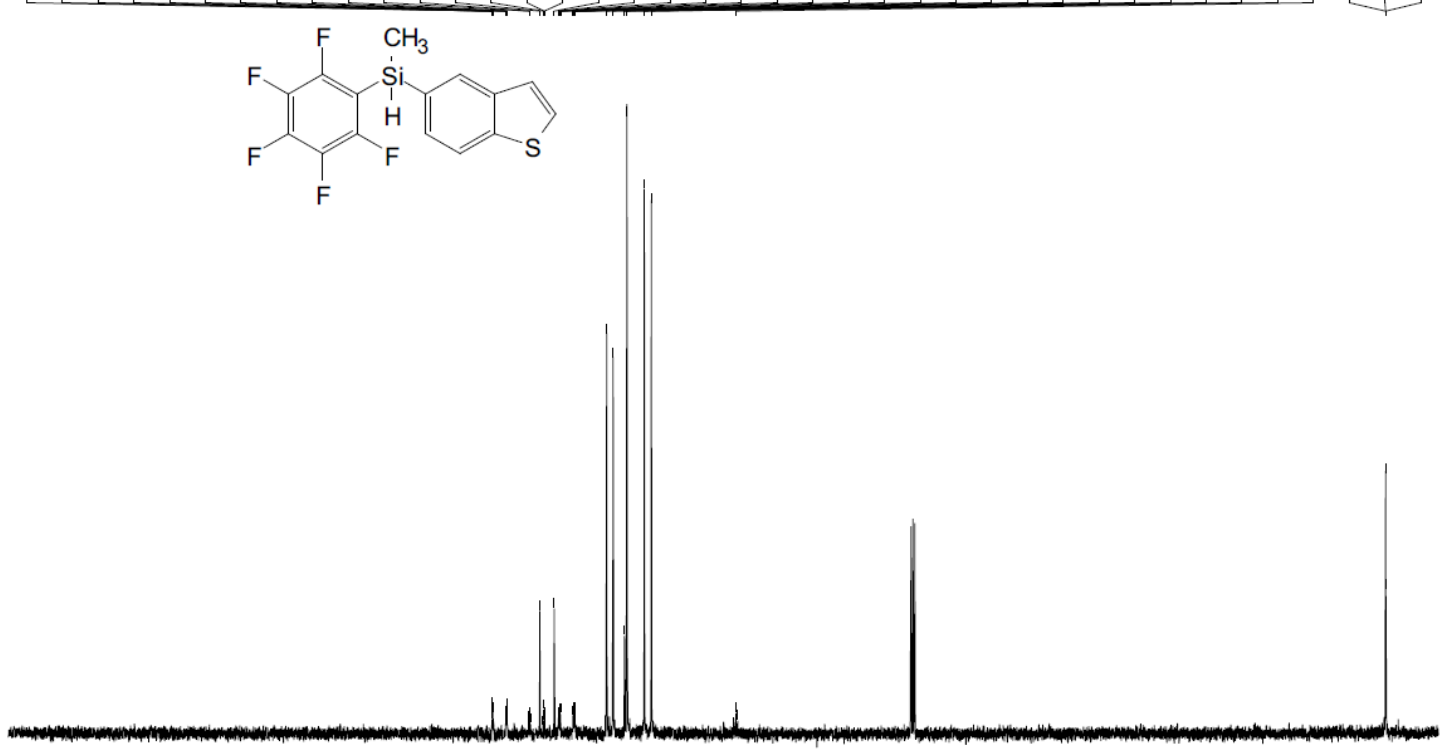

$\begin{array}{lllllllllllllllllllllllll}230 & 220 & 210 & 200 & 190 & 180 & 170 & 160 & 150 & 140 & 130 & 120 & 110 & 100 & 90 & 80 & 70 & 60 & 50 & 40 & 30 & 20 & 10 & 0 & -10\end{array}$

${ }^{13} \mathrm{C} \mathrm{NMR}\left(101 \mathrm{MHz}, \mathrm{CDCl}_{3}\right)$ of $\mathbf{5 g}$ 


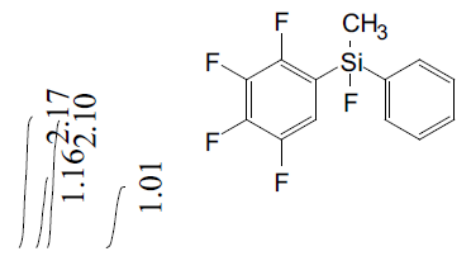

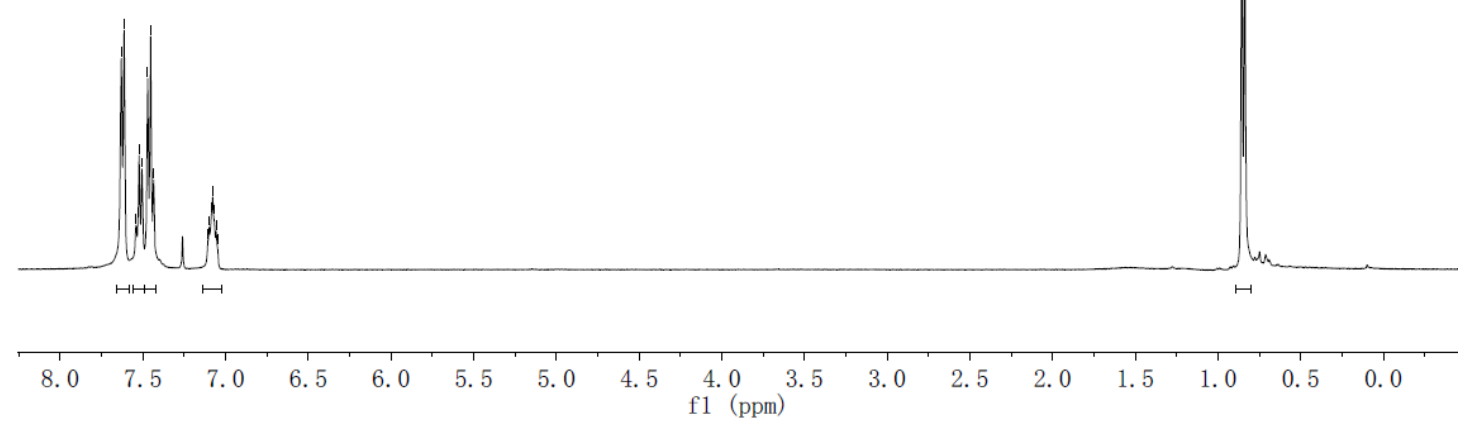

${ }^{1} \mathrm{H} \mathrm{NMR}\left(400 \mathrm{MHz}, \mathrm{CDCl}_{3}\right)$ of $\mathbf{3 a}$

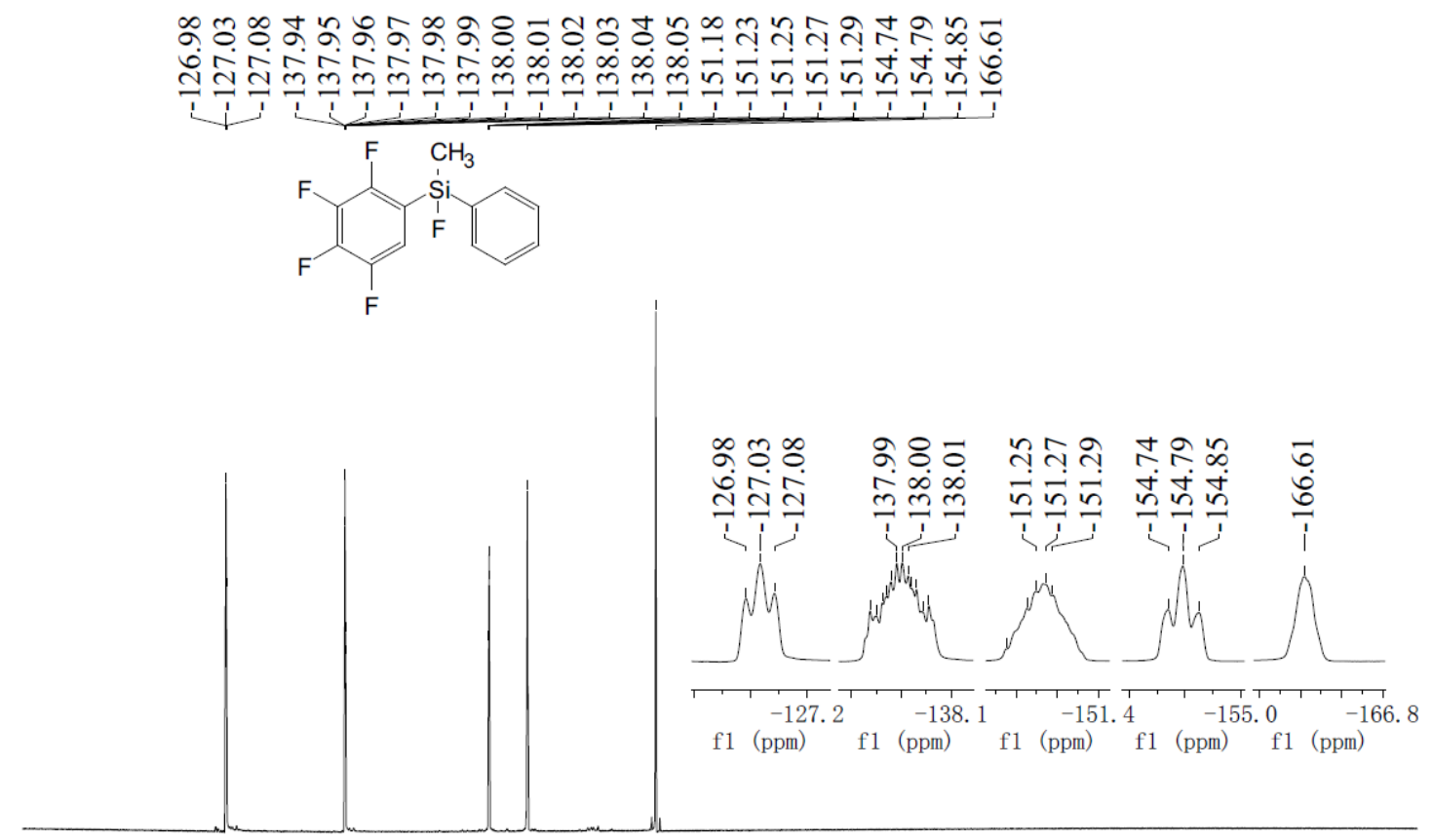

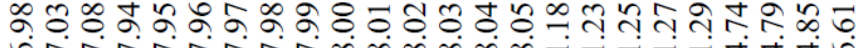

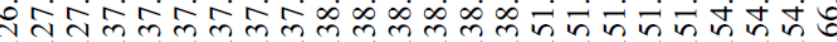

\begin{tabular}{|c|c|c|c|c|c|c|c|c|c|c|c|}
\hline-115 & -125 & -135 & -145 & -155 & -165 & $\begin{array}{l}-175 \\
(\mathrm{ppm})\end{array}$ & -185 & -195 & -205 & -215 & -225 \\
\hline
\end{tabular}

${ }^{19} \mathrm{~F} \mathrm{NMR}\left(376 \mathrm{MHz}, \mathrm{CDCl}_{3}\right)$ of $\mathbf{3 a}$ 
<smiles>C[Si](C)(c1ccccc1F)c1cccc(F)c1F</smiles>

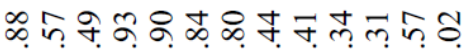

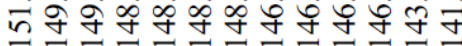

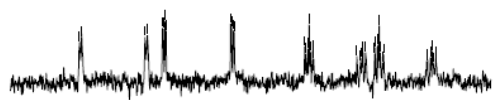

$\begin{array}{lllllllll}154 & 152 & 150 & 148 & 146 & 144 & 142 & 140 & 138\end{array}$ f1 (ppm)

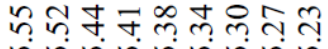

ำดลำ
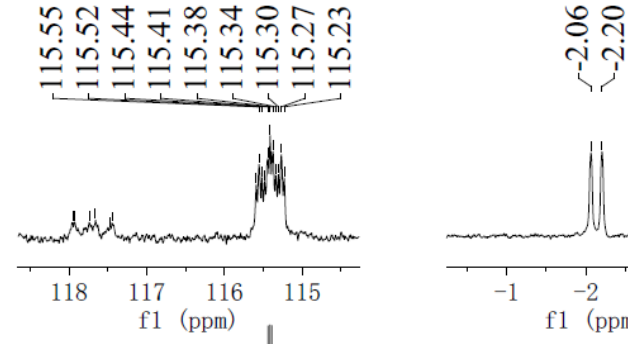

Uلب
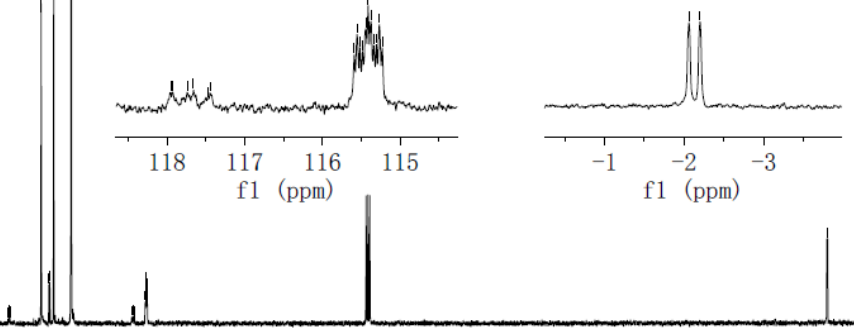

$\begin{array}{llllllllllllllllllllllllll}230 & 220 & 210 & 200 & 190 & 180 & 170 & 160 & 150 & 140 & 130 & 120 & 110 & 100 & 90 & 80 & 70 & 60 & 50 & 40 & 30 & 20 & 10 & 0 & -10\end{array}$

${ }^{13} \mathrm{C} \mathrm{NMR}\left(101 \mathrm{MHz}, \mathrm{CDCl}_{3}\right)$ of $\mathbf{3 a}$ 
<smiles>COc1ccccc1[Si](C)(c1ccccc1)c1cc(F)c(F)c(F)c1F</smiles>
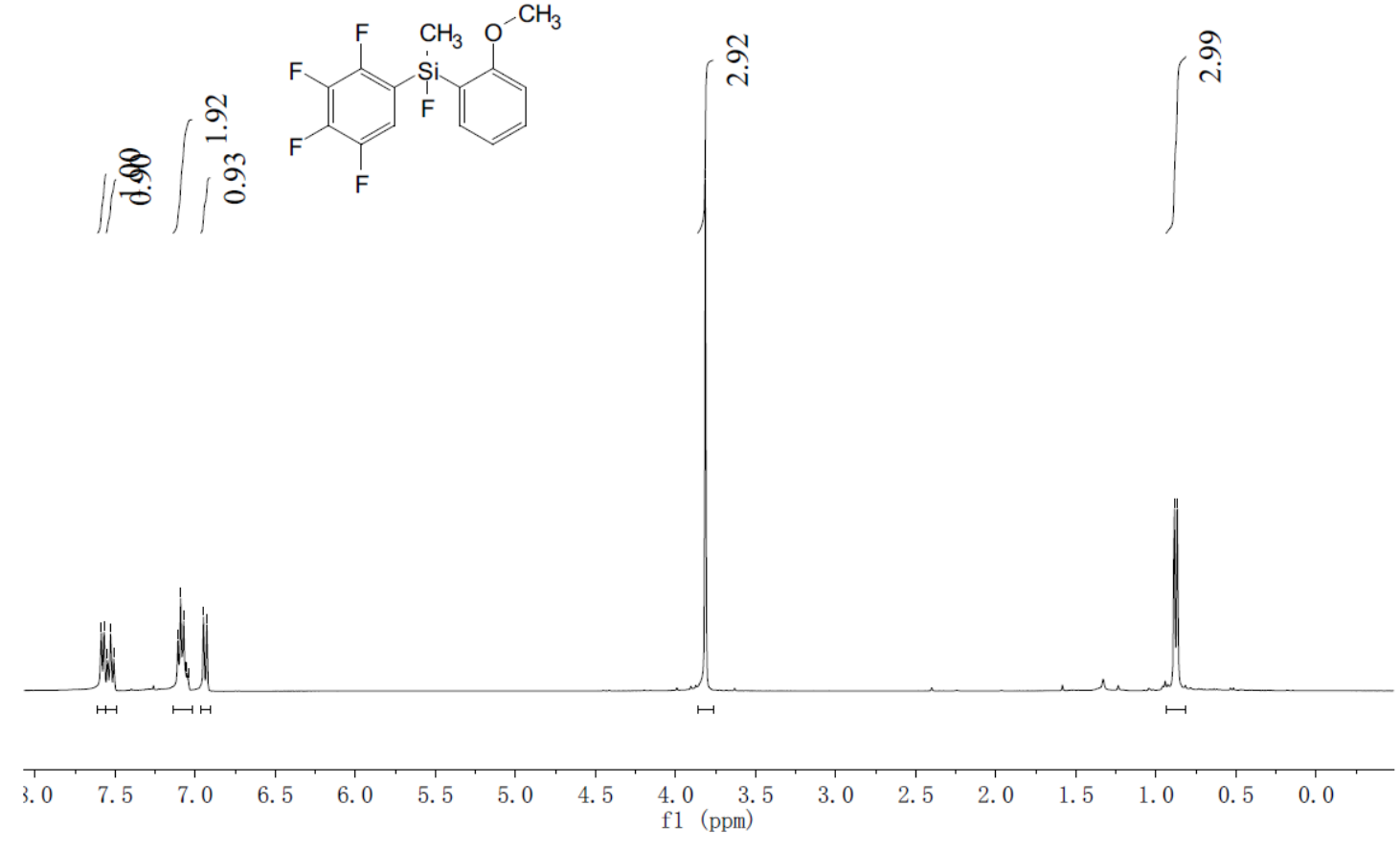

${ }^{1} \mathrm{H} \mathrm{NMR}\left(400 \mathrm{MHz}, \mathrm{CDCl}_{3}\right)$ of $\mathbf{3 b}$

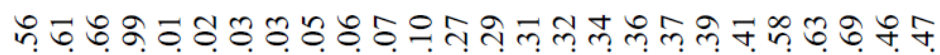

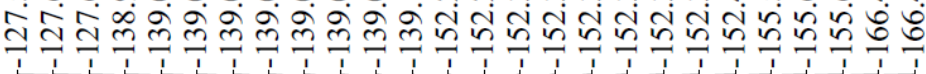<smiles>COc1ccccc1[Si](C)(C)c1cc(F)c(F)c(F)c1F</smiles>

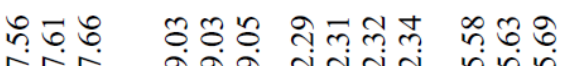

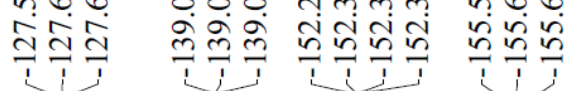

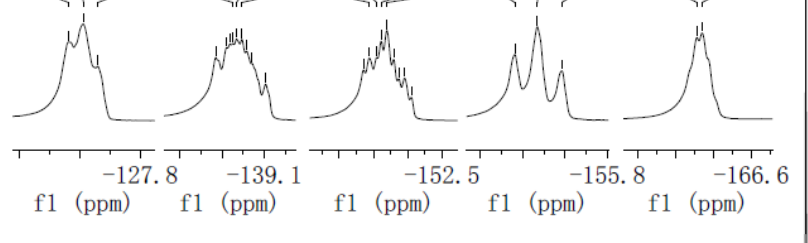

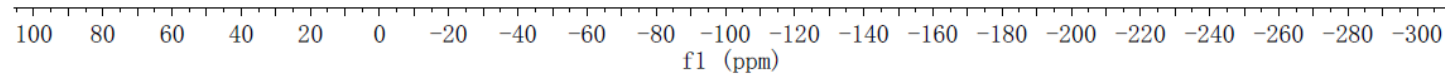
${ }^{19} \mathrm{~F} \mathrm{NMR}\left(376 \mathrm{MHz}, \mathrm{CDCl}_{3}\right)$ of $\mathbf{3 b}$ 


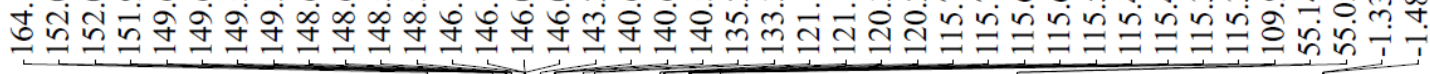<smiles>COc1ccccc1[Si](C)(c1ccccc1)c1cc(F)c(F)c(F)c1F</smiles>

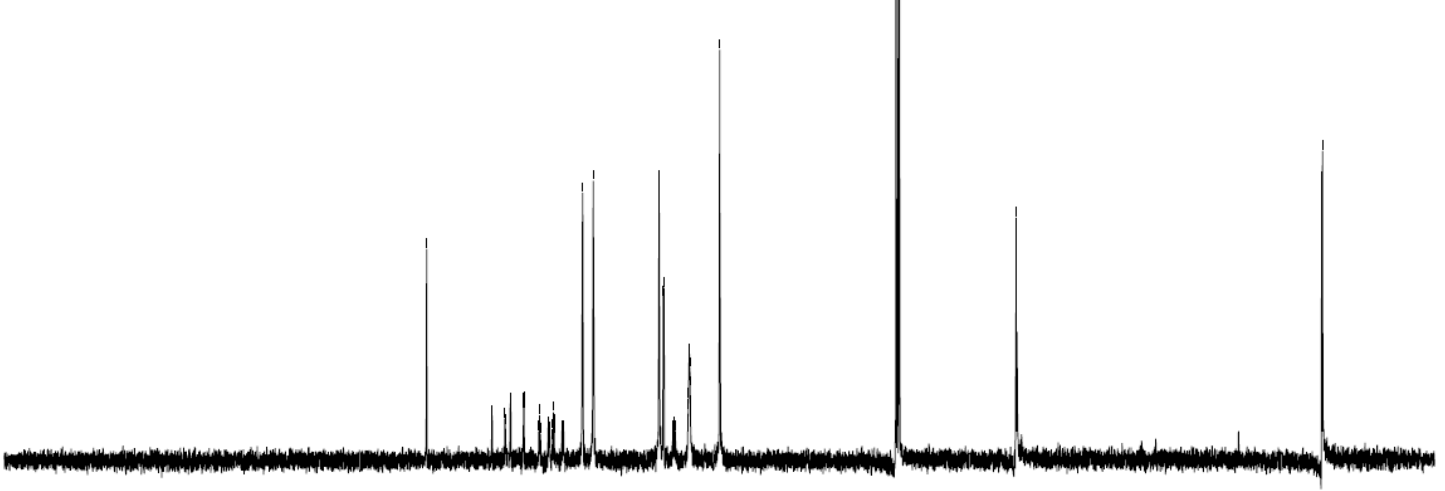

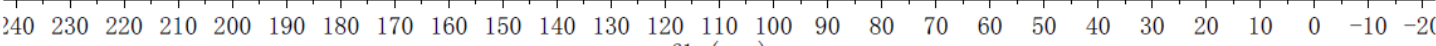
f1 (ppm)

$\left.{ }^{13} \mathrm{C} \mathrm{NMR} \mathrm{(101} \mathrm{MHz,} \mathrm{CDCl}_{3}\right)$ of $\mathbf{3 b}$ 

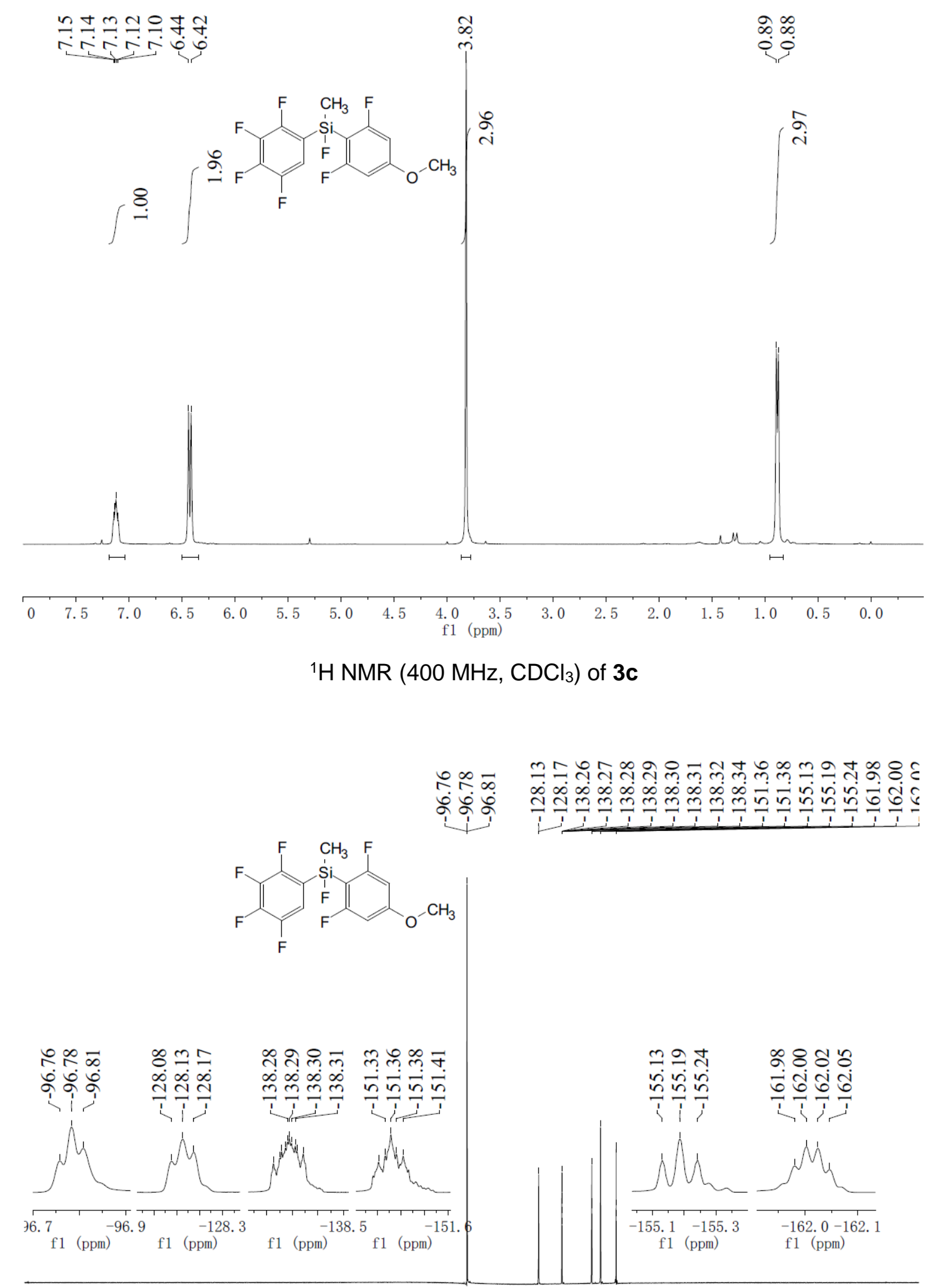

\begin{tabular}{llllllllllllllllllllll}
\hline 90 & 70 & 50 & 30 & 10 & -10 & -30 & -50 & -70 & -90 & -110 & -130 & -150 & -170 & -190 & -210 & -230 & -250 & -270 & -291 \\
\hline
\end{tabular} ${ }^{19} \mathrm{~F} \mathrm{NMR}\left(376 \mathrm{MHz}, \mathrm{CDCl}_{3}\right)$ of $\mathbf{3 c}$ 


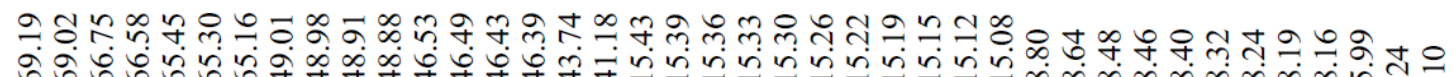

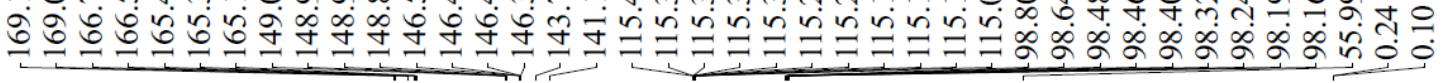<smiles>COc1cc(F)c([Si](C)(C)c2cc(F)c(F)c(F)c2F)c(F)c1</smiles>

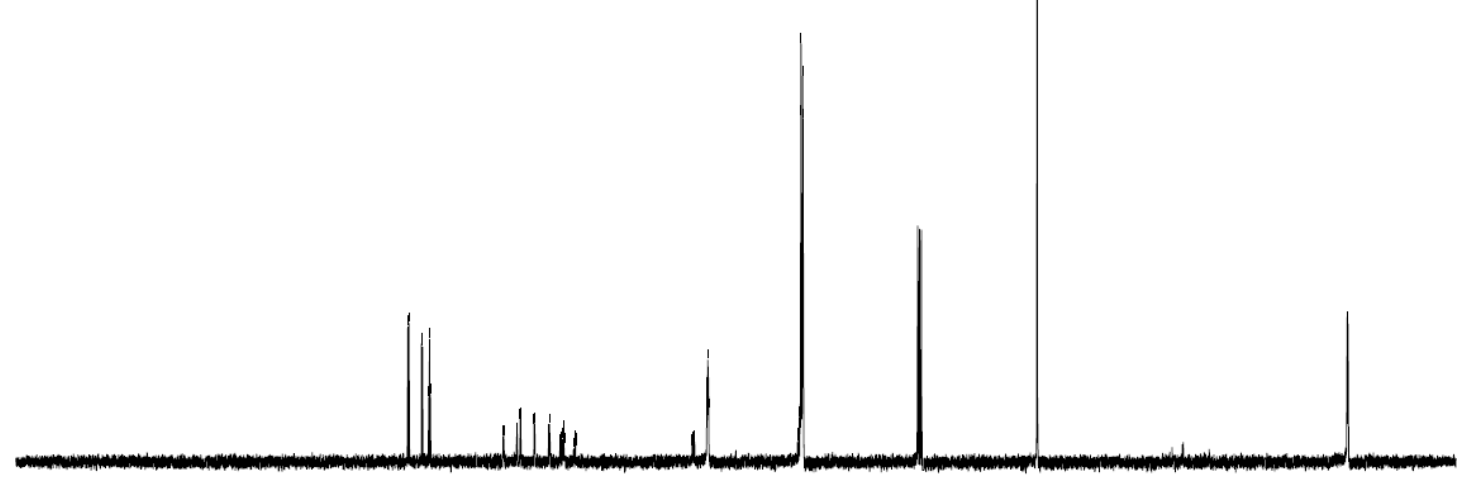

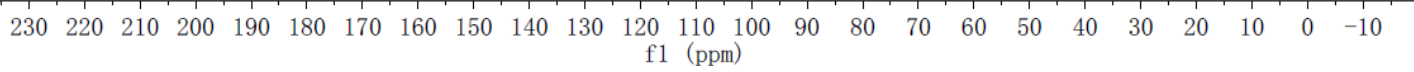
${ }^{13} \mathrm{C} \mathrm{NMR}\left(101 \mathrm{MHz}, \mathrm{CDCl}_{3}\right)$ of $3 \mathrm{c}$ 


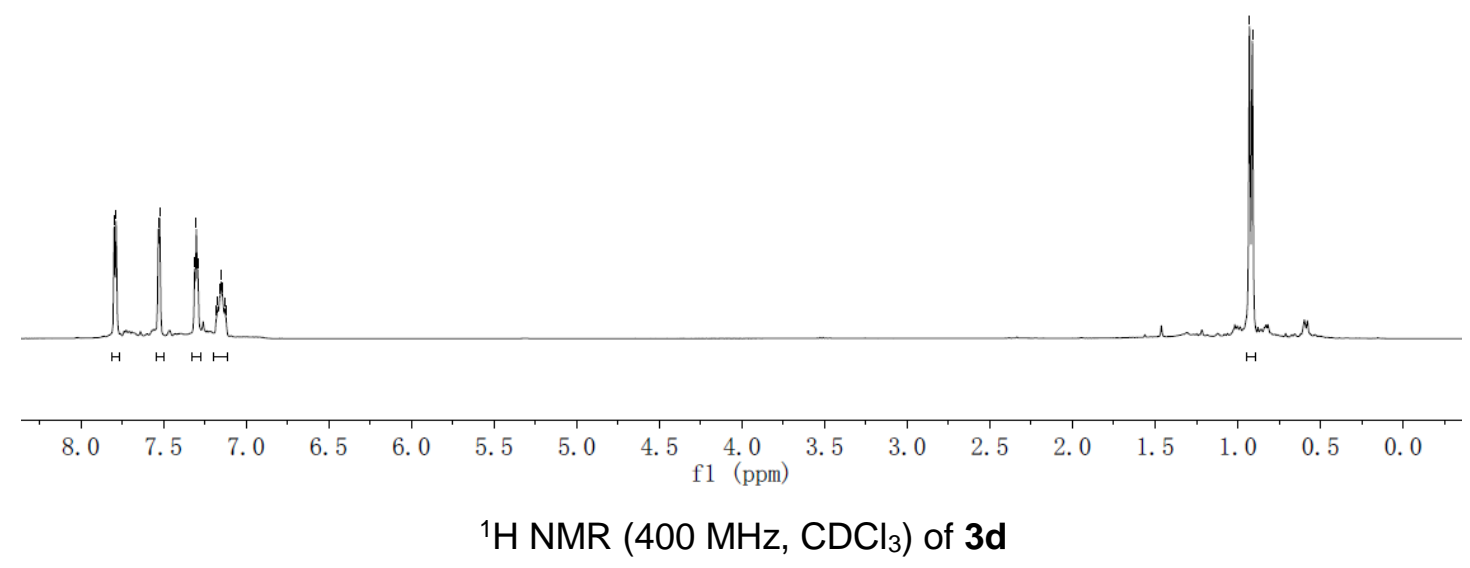<smiles>C[Si](c1cccs1)(c1cccs1)c1ccc(F)c(F)c1F</smiles>

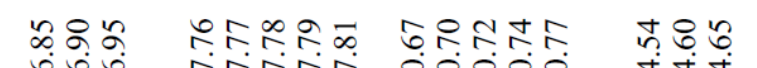

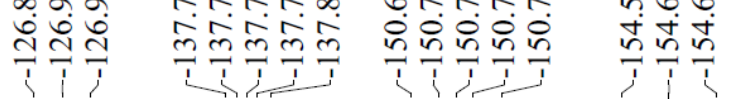
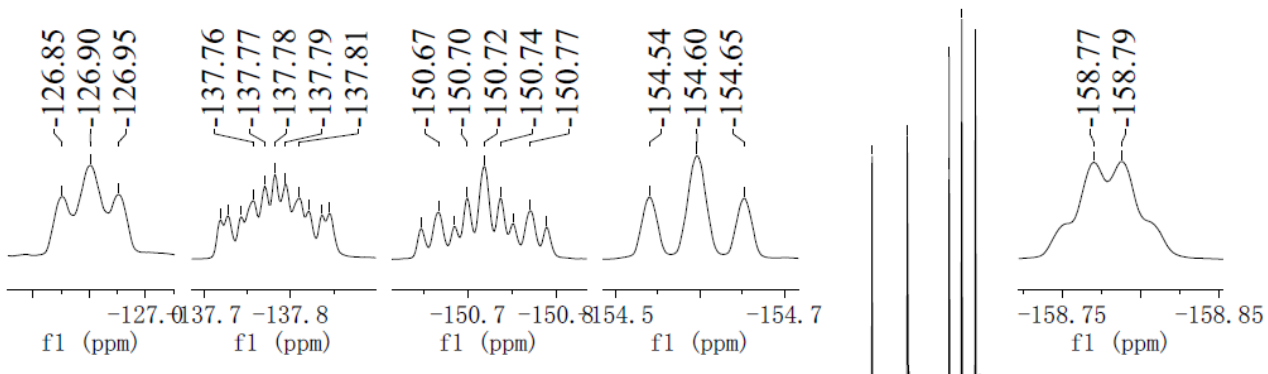

$\begin{array}{rrrrrrr}140 & 120 & 100 & 80 & 60 & 40 & 20\end{array}$

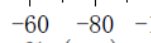
f1 (ppm)

$\left.{ }^{19} \mathrm{~F} \mathrm{NMR} \mathrm{(376} \mathrm{MHz,} \mathrm{CDCl}_{3}\right)$ of $\mathbf{3 d}$ 
<smiles>Cc1ccc(-c2cccs2)cc1F</smiles>

${ }^{13} \mathrm{C} \mathrm{NMR}\left(101 \mathrm{MHz}, \mathrm{CDCl}_{3}\right)$ of $\mathbf{3 d}$ 

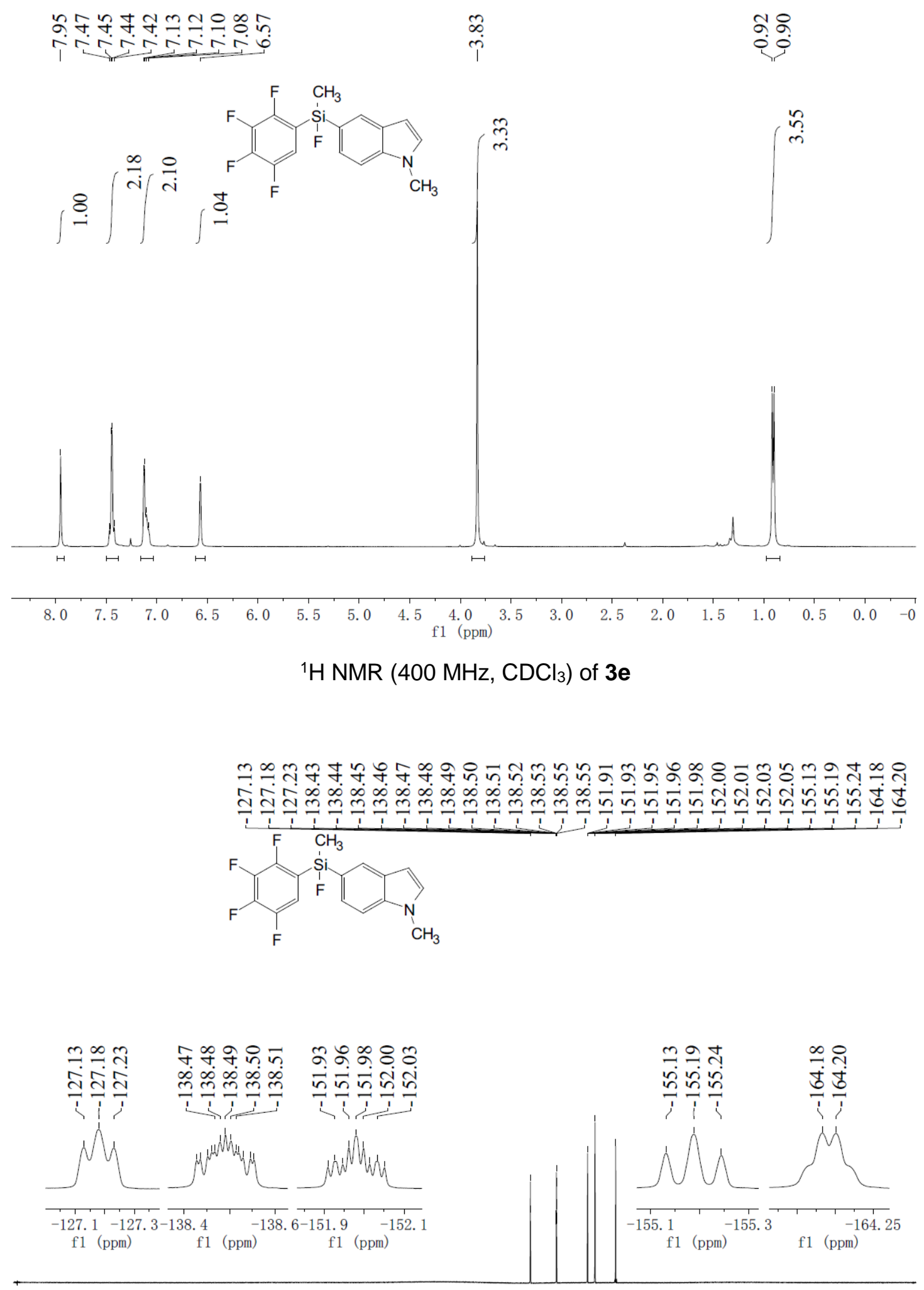

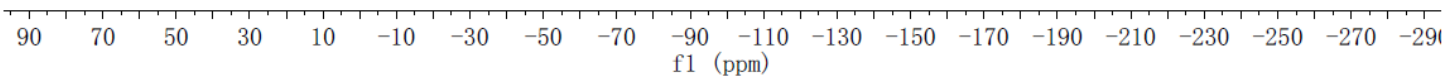
${ }^{19} \mathrm{~F} \mathrm{NMR}\left(376 \mathrm{MHz}, \mathrm{CDCl}_{3}\right)$ of $\mathbf{3 e}$ 


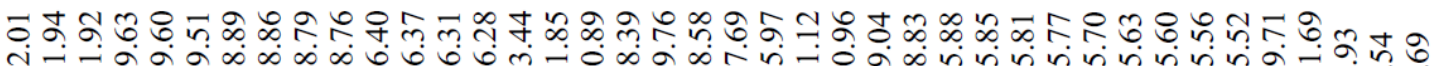

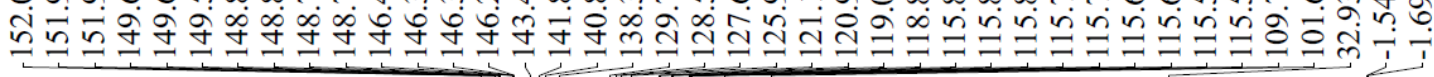

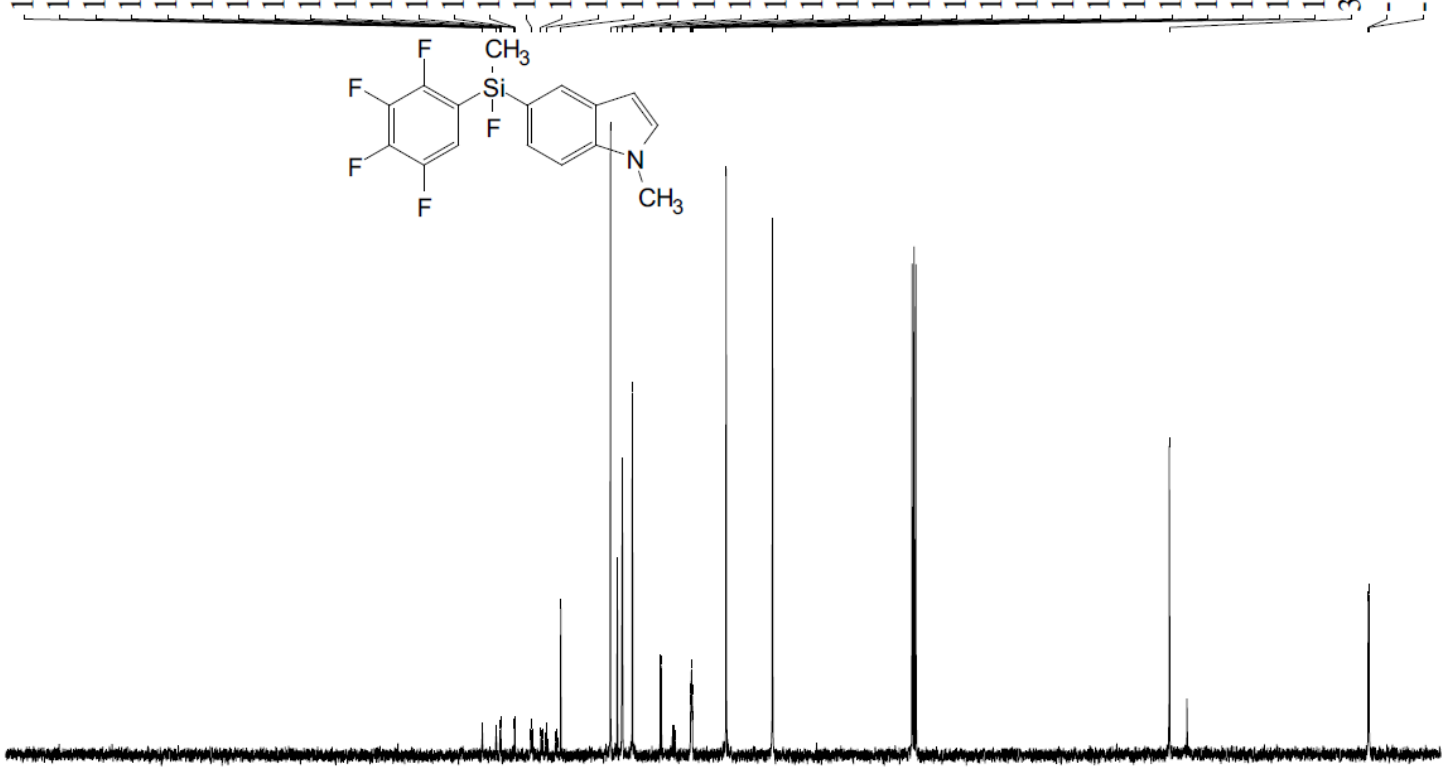

$\begin{array}{lllllllllllllllllllllllllllll}230 & 220 & 210 & 200 & 190 & 180 & 170 & 160 & 150 & 140 & 130 & 120 & 110 & 100 & 90 & 80 & 70 & 60 & 50 & 40 & 30 & 20 & 10 & 0 & -10\end{array}$

${ }^{13} \mathrm{C} \mathrm{NMR}\left(101 \mathrm{MHz}, \mathrm{CDCl}_{3}\right)$ of $3 e$ 


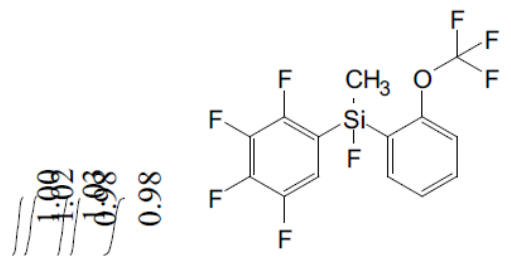

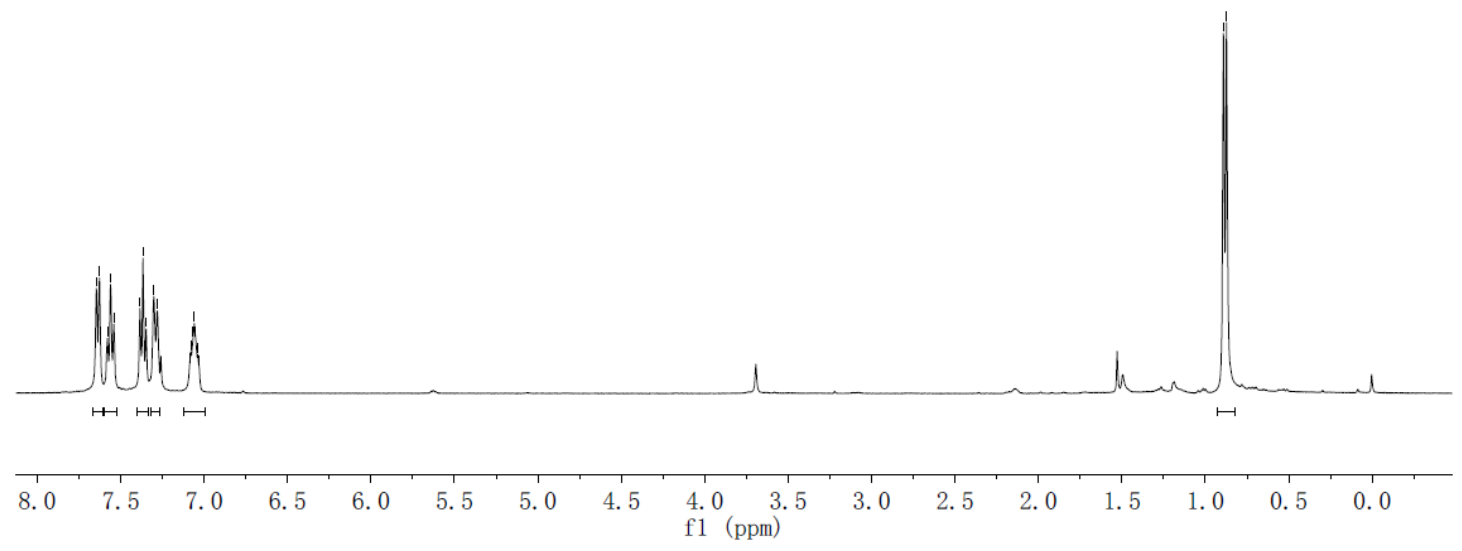

${ }^{1} \mathrm{H} \mathrm{NMR}\left(400 \mathrm{MHz}, \mathrm{CDCl}_{3}\right)$ of $\mathbf{3 f}$

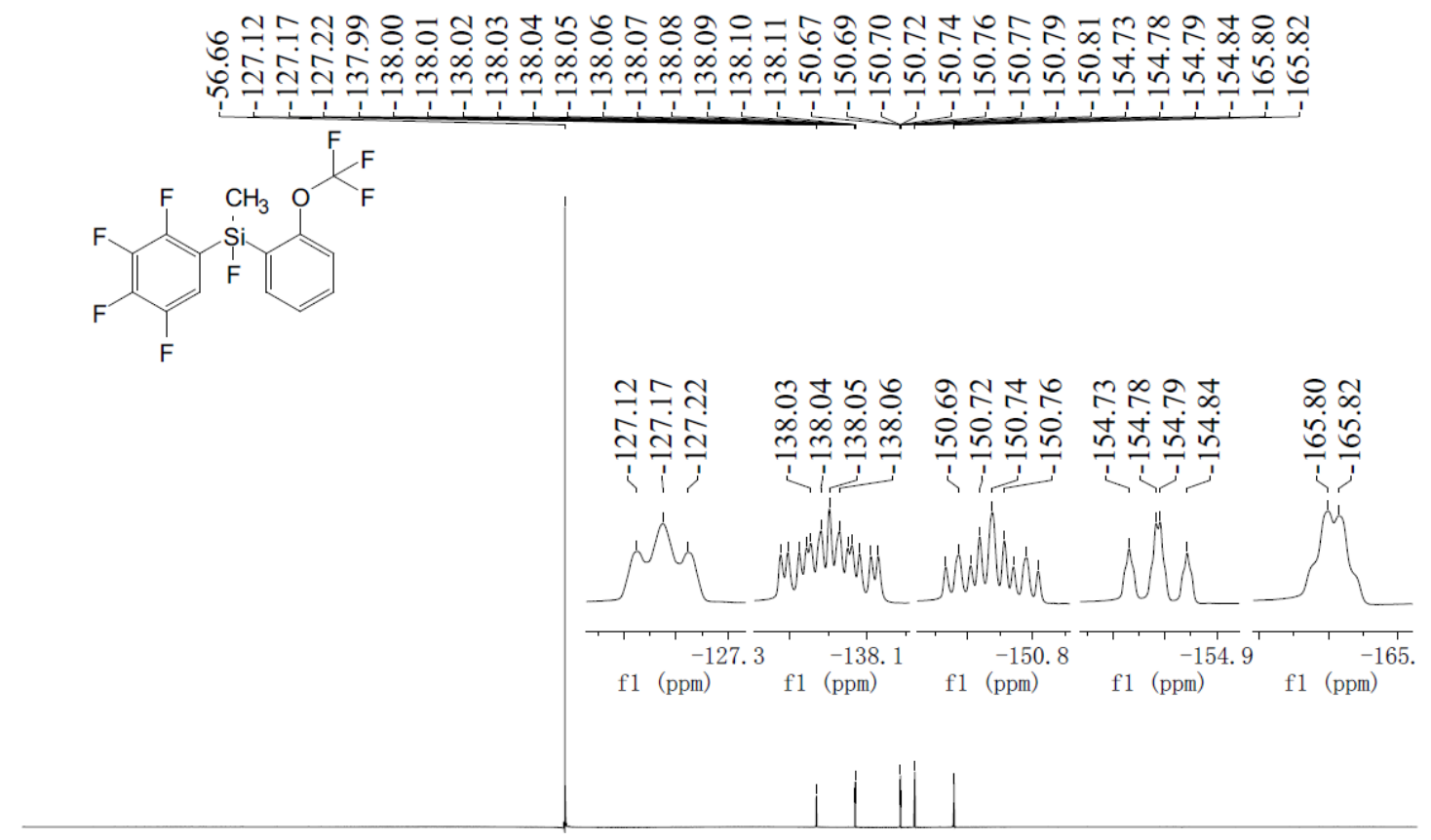

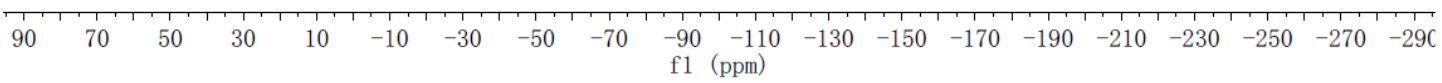

${ }^{19} \mathrm{~F} \mathrm{NMR}\left(376 \mathrm{MHz}, \mathrm{CDCl}_{3}\right)$ of $\mathbf{3 f}$ 


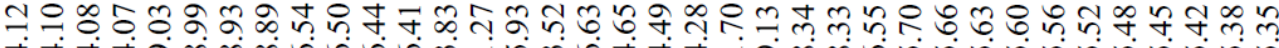

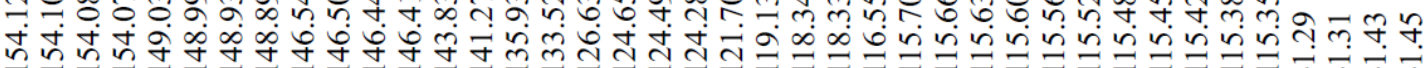

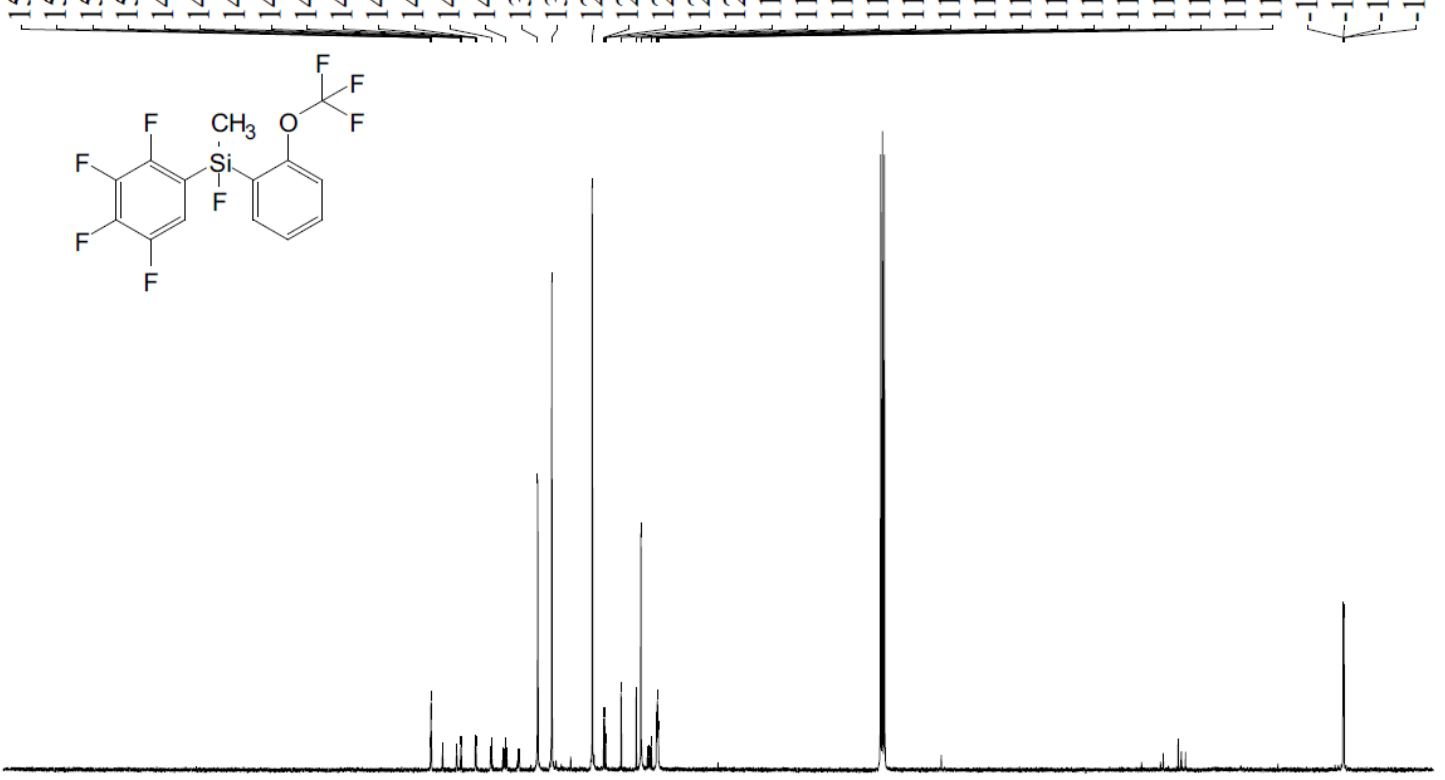

$\begin{array}{llllllllllllllllllllllll}220 & 210 & 200 & 190 & 180 & 170 & 160 & 150 & 140 & 130 & 120 & 110 & 100 & 90 & 80 & 70 & 60 & 50 & 40 & 30 & 20 & 10 & 0 & -10\end{array}$ f1 (ppm)

${ }^{13} \mathrm{C} \mathrm{NMR}\left(101 \mathrm{MHz}, \mathrm{CDCl}_{3}\right)$ of $\mathbf{3 f}$ 

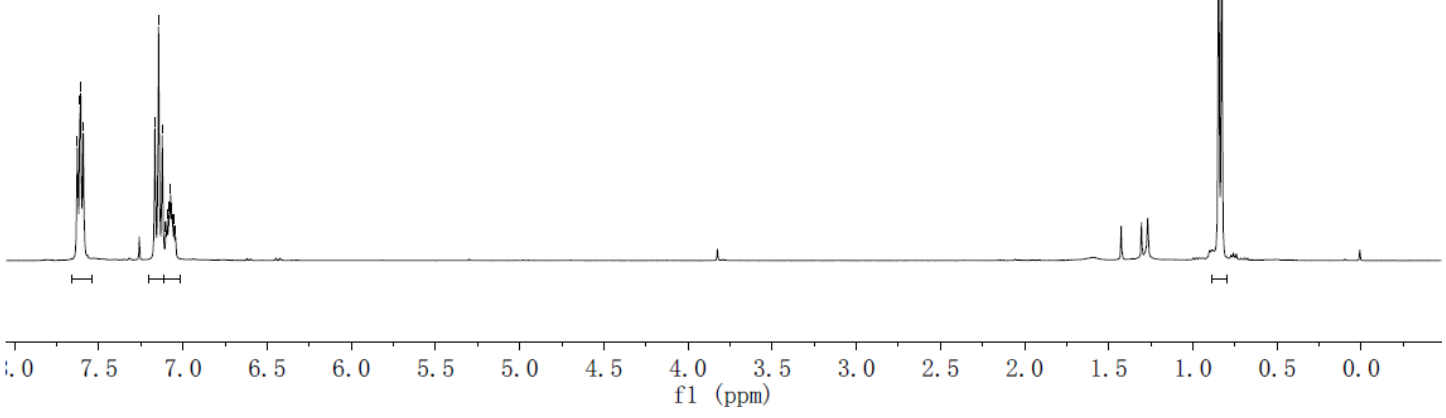

${ }^{1} \mathrm{H}$ NMR $\left(400 \mathrm{MHz}, \mathrm{CDCl}_{3}\right)$ of $\mathbf{3 g}$

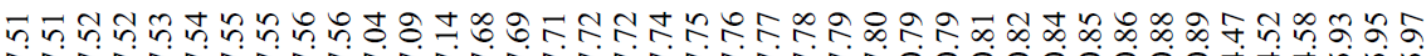

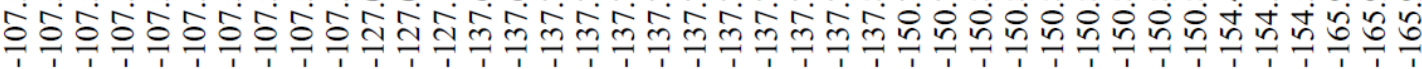<smiles>C[Si](c1ccc(F)cc1)(c1ccc(F)c(F)c1)c1cc(F)c(F)c(F)c1F</smiles>

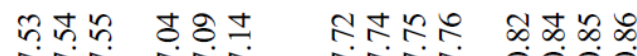

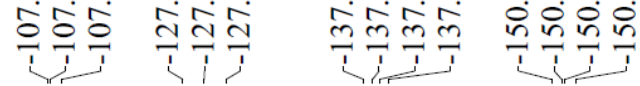
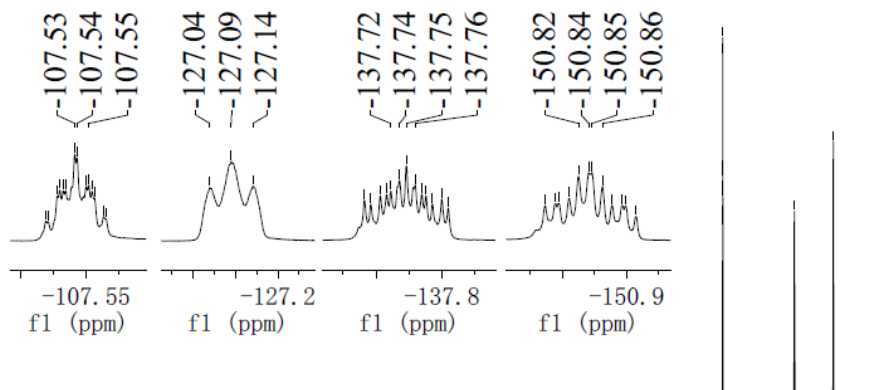

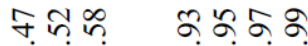

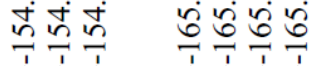

i? ?

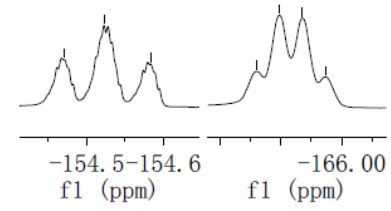

$\mathrm{fl}(\mathrm{ppm}) \quad \mathrm{f} 1(\mathrm{ppm}) \quad \mathrm{f} 1(\mathrm{ppm}) \quad \mathrm{f} 1(\mathrm{ppm})$

f1 (ppm)

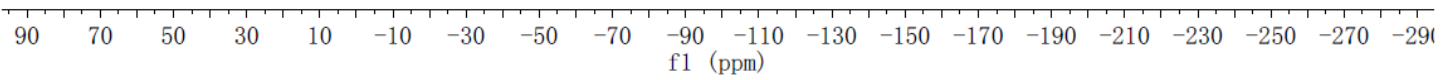

$\left.{ }^{19} \mathrm{~F} \mathrm{NMR} \mathrm{(376} \mathrm{MHz,} \mathrm{CDCl}_{3}\right)$ of $\mathbf{3 g}$ 


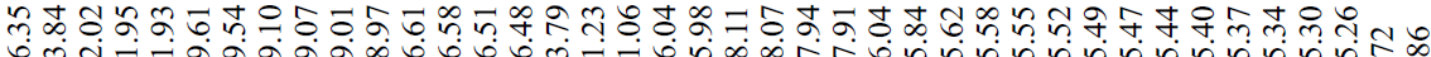
은<smiles>C[Si](c1ccc(F)cc1)(c1ccccc1F)c1ccc(F)c(F)c1F</smiles>

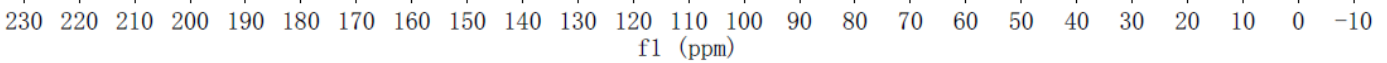
${ }^{13} \mathrm{C}$ NMR $\left(101 \mathrm{MHz}, \mathrm{CDCl}_{3}\right)$ of $\mathbf{3 g}$ 
<smiles>Cc1ccc([Si](C)(F)c2cc(F)c(F)c(F)c2F)cc1</smiles>
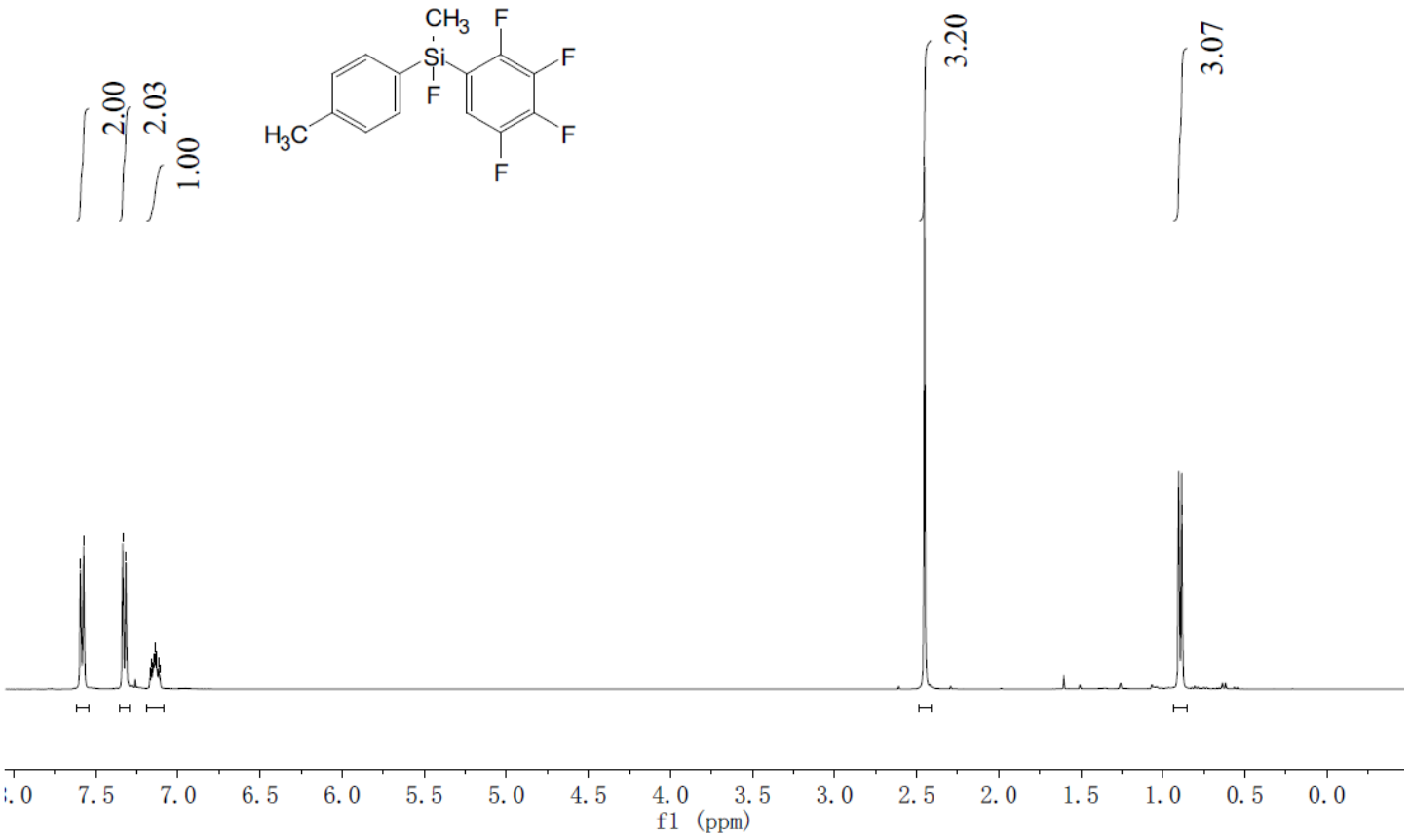

${ }^{1} \mathrm{H}$ NMR $\left(400 \mathrm{MHz}, \mathrm{CDCl}_{3}\right)$ of $\mathbf{3 h}$

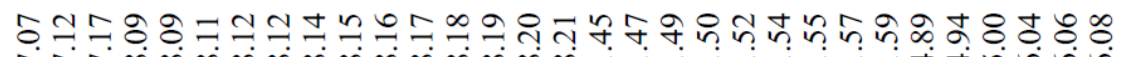

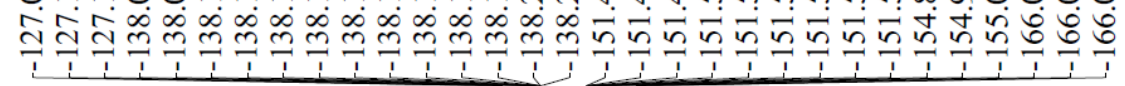<smiles>Cc1ccc([Si](C)(F)c2cc(F)c(F)c(F)c2F)cc1</smiles>

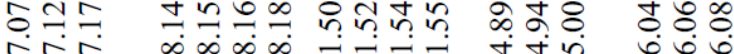

तิ수
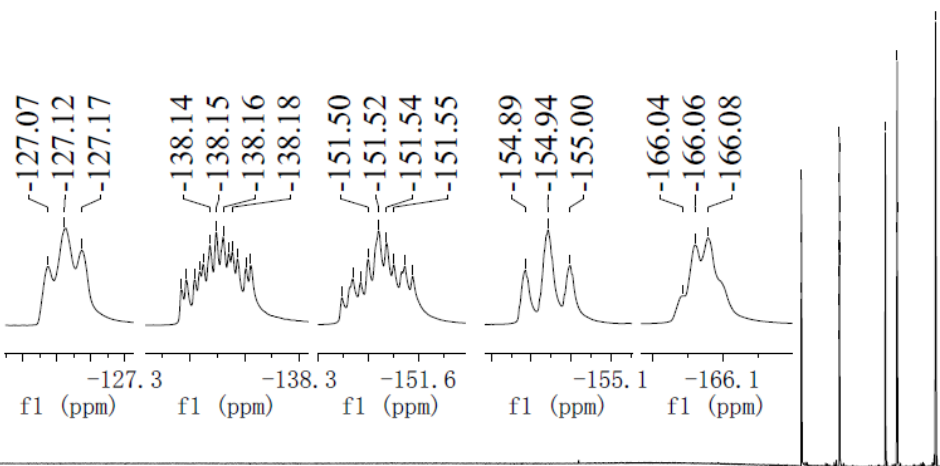

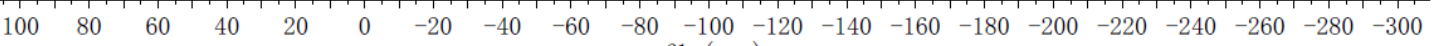

${ }^{19} \mathrm{~F} \mathrm{NMR}\left(376 \mathrm{MHz}, \mathrm{CDCl}_{3}\right)$ of $\mathbf{3 h}$ 


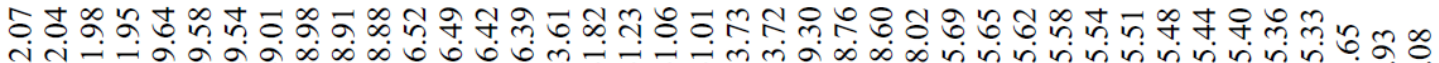
nู่

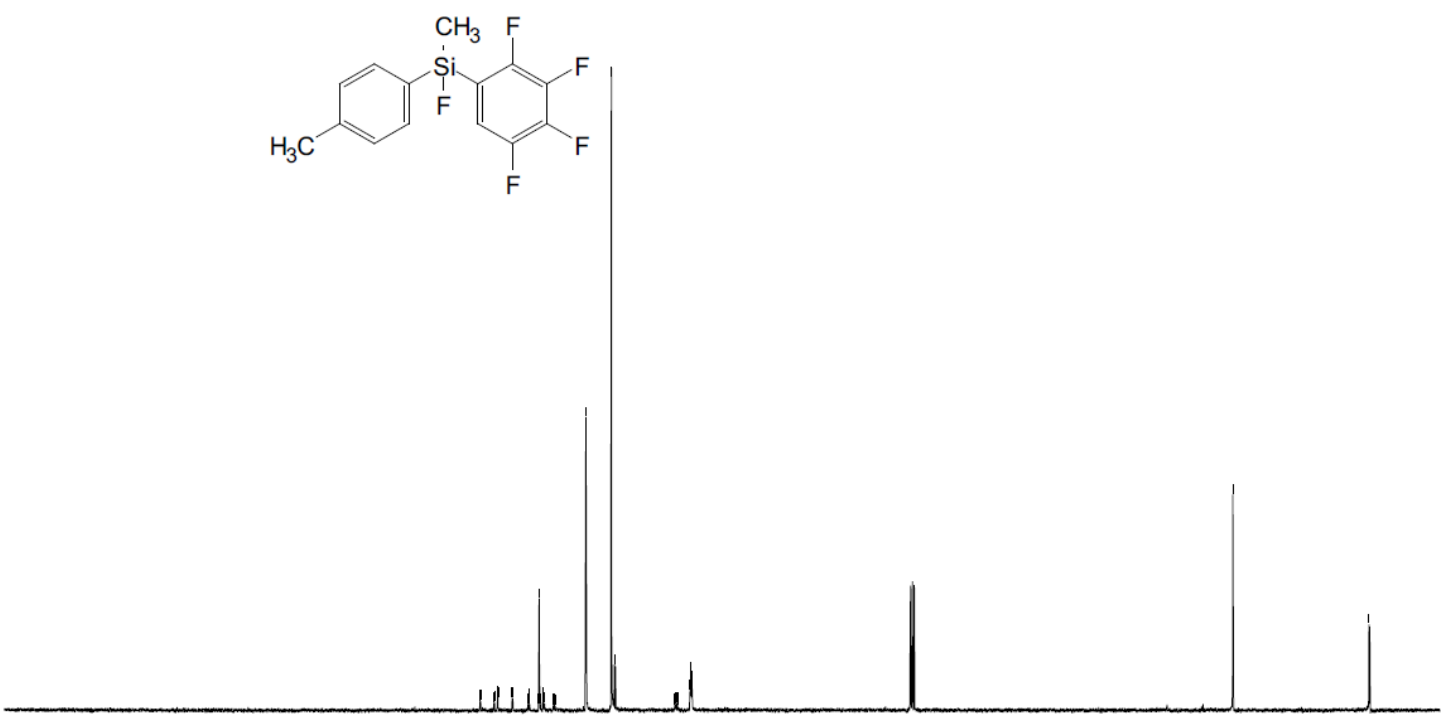

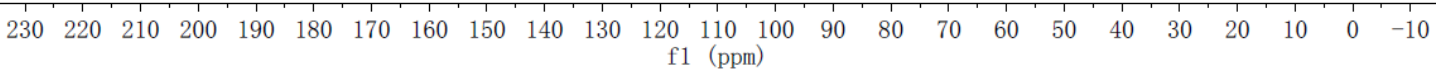

${ }^{13} \mathrm{C} \mathrm{NMR}\left(101 \mathrm{MHz}, \mathrm{CDCl}_{3}\right)$ of $\mathbf{3 h}$ 


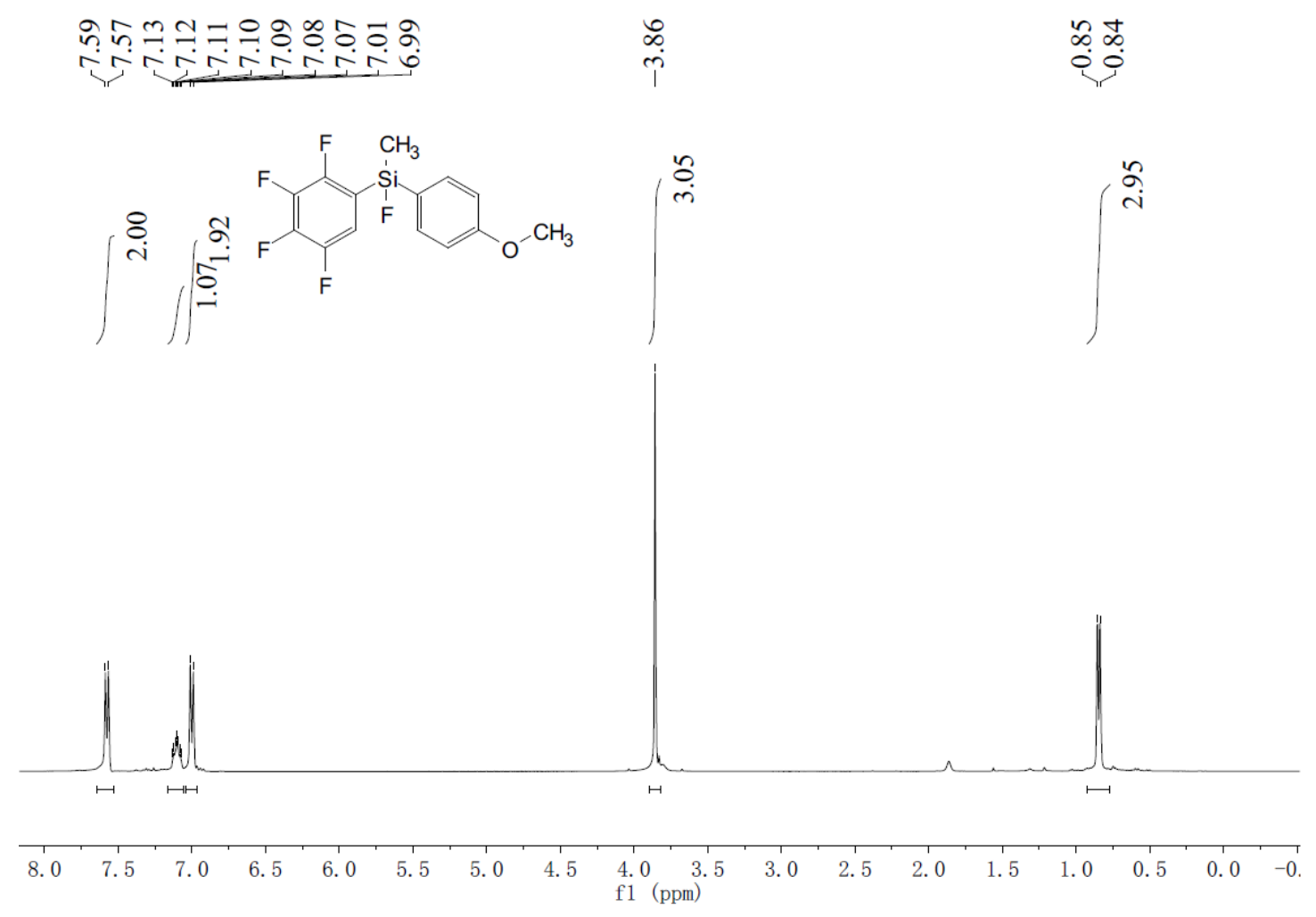

${ }^{1} \mathrm{H}$ NMR $\left(400 \mathrm{MHz}, \mathrm{CDCl}_{3}\right)$ of $\mathbf{3 i}$

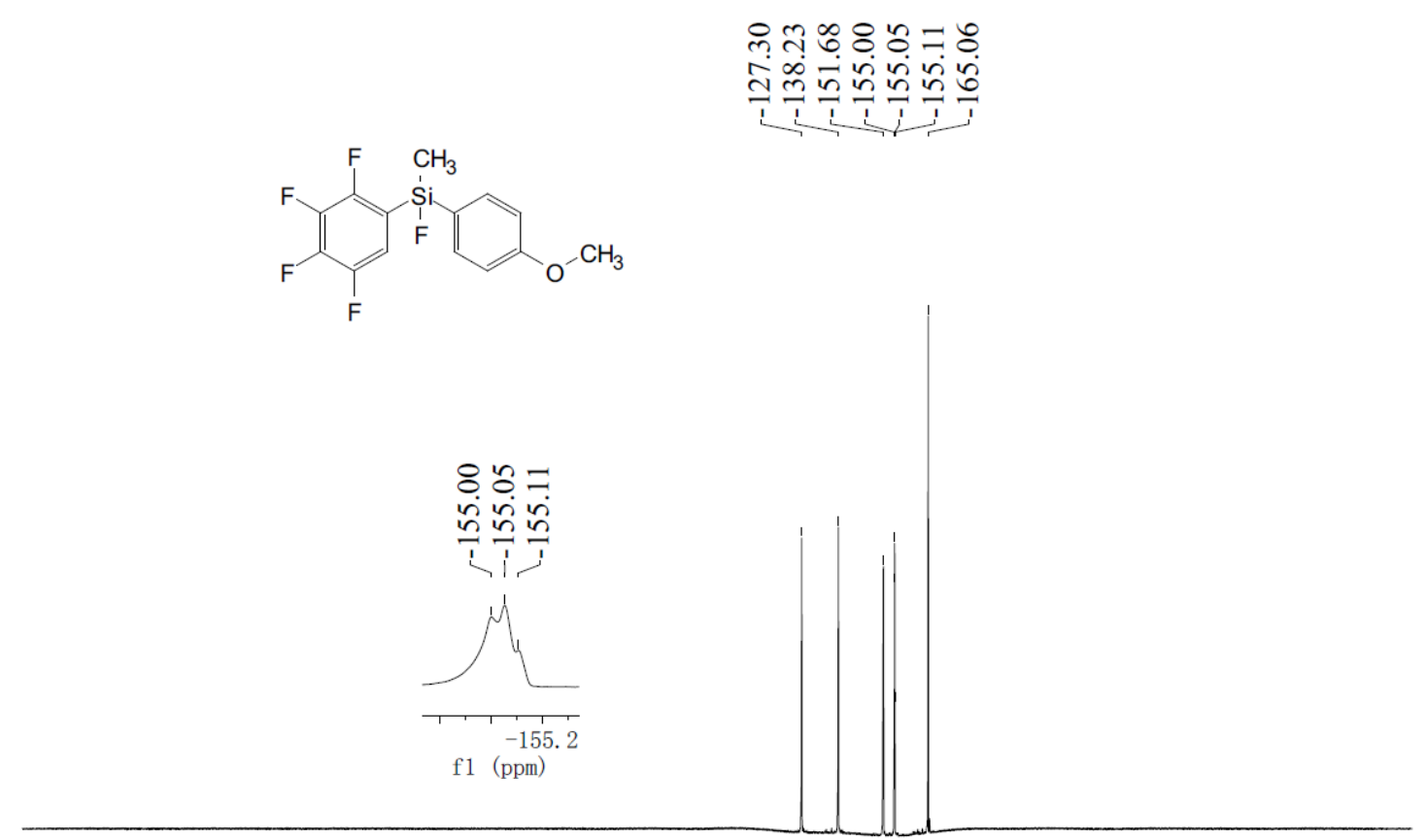

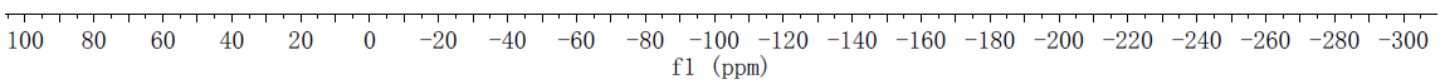

${ }^{19} \mathrm{~F} \mathrm{NMR} \mathrm{(376} \mathrm{MHz,} \mathrm{CDCl}_{3}$ ) of $\mathbf{3 i}$ 


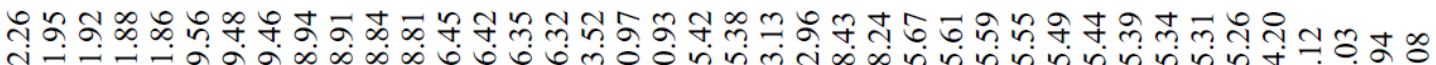

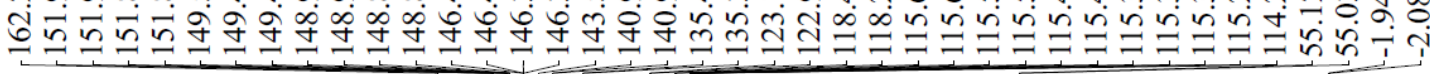<smiles>COc1ccc([Si](C)(F)c2cc(F)c(F)c(F)c2F)cc1</smiles>

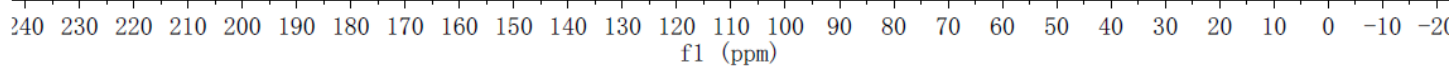
${ }^{13} \mathrm{C}$ NMR $\left(101 \mathrm{MHz}, \mathrm{CDCl}_{3}\right)$ of $\mathbf{3 i}$ 
<smiles>CN(C)c1ccc([Si](C)(C)c2cc(F)c(F)c(F)c2F)cc1</smiles>
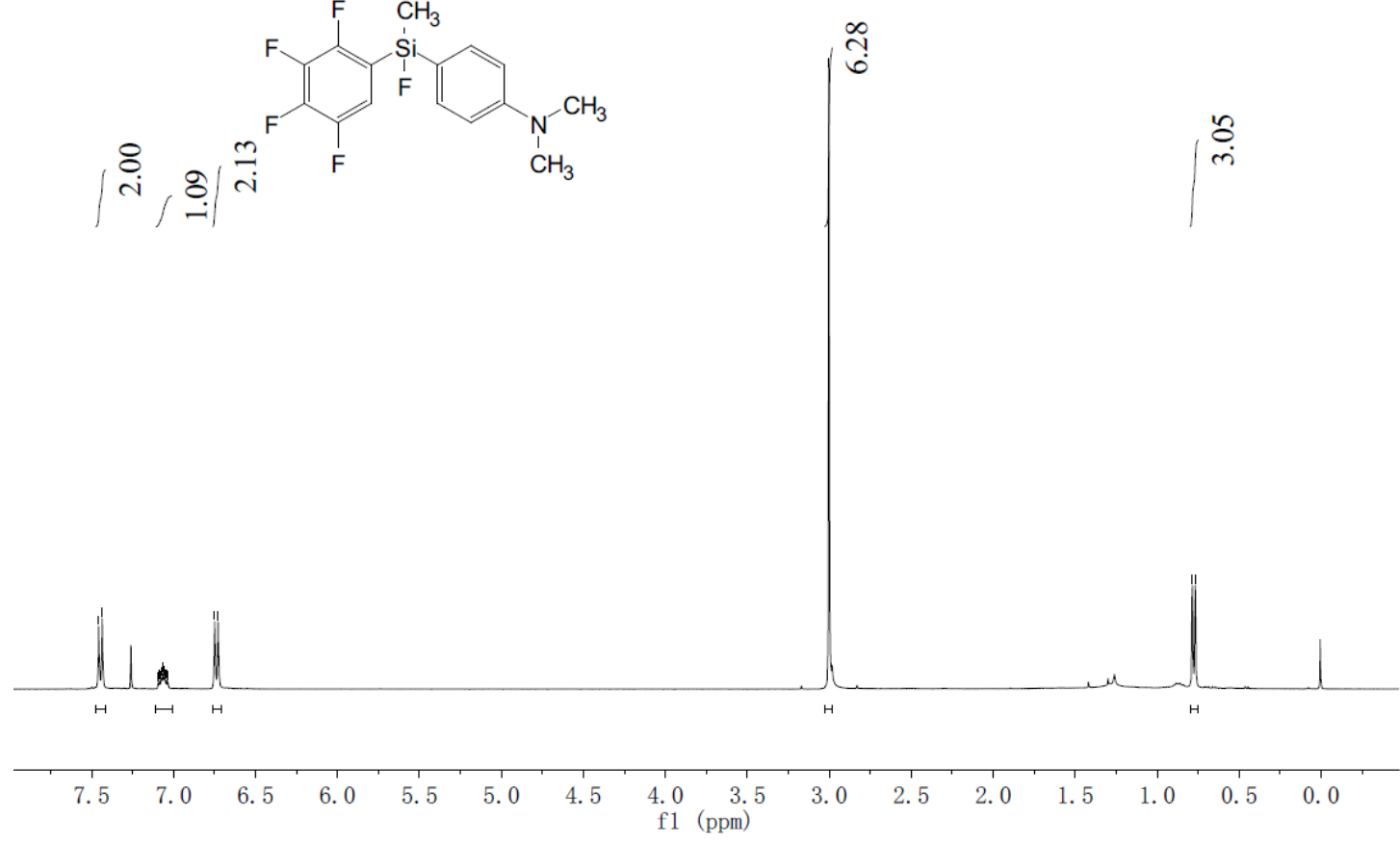

${ }^{1} \mathrm{H} \mathrm{NMR}\left(400 \mathrm{MHz}, \mathrm{CDCl}_{3}\right)$ of $\mathbf{3 j}$

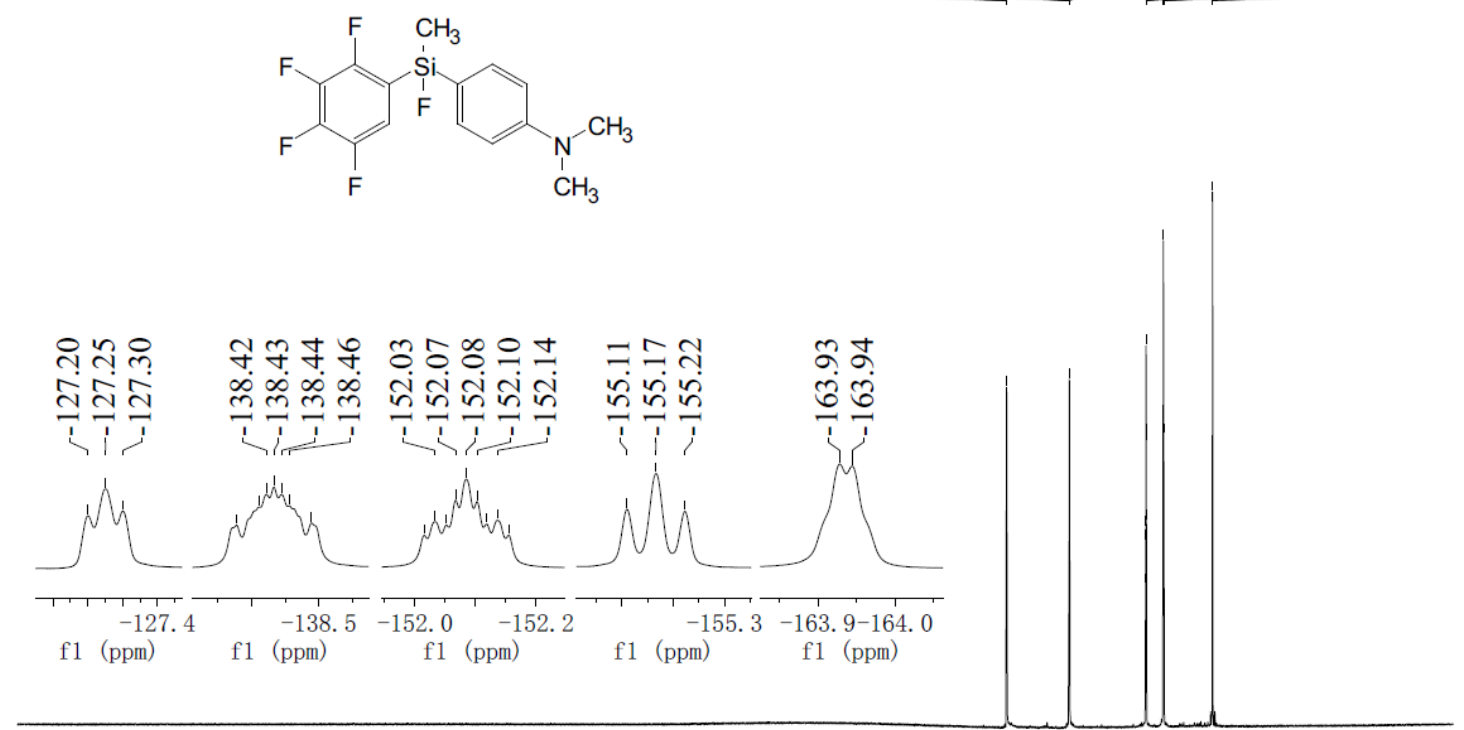

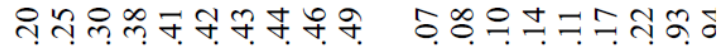

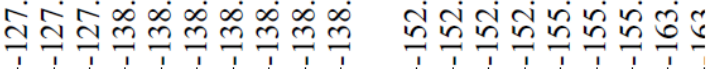

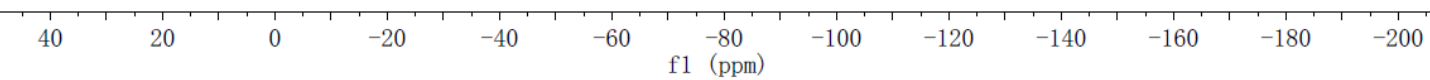

${ }^{19} \mathrm{~F} \mathrm{NMR}\left(376 \mathrm{MHz}, \mathrm{CDCl}_{3}\right)$ of $\mathbf{3 j}$ 


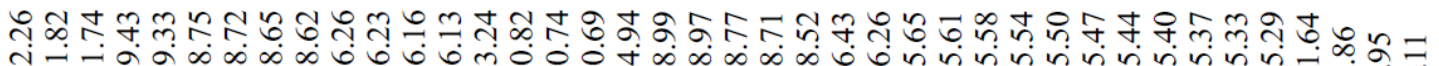

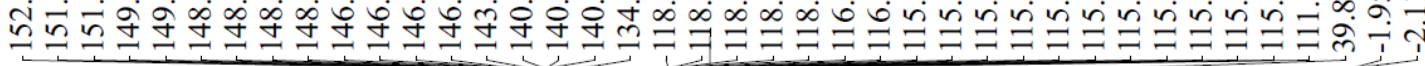

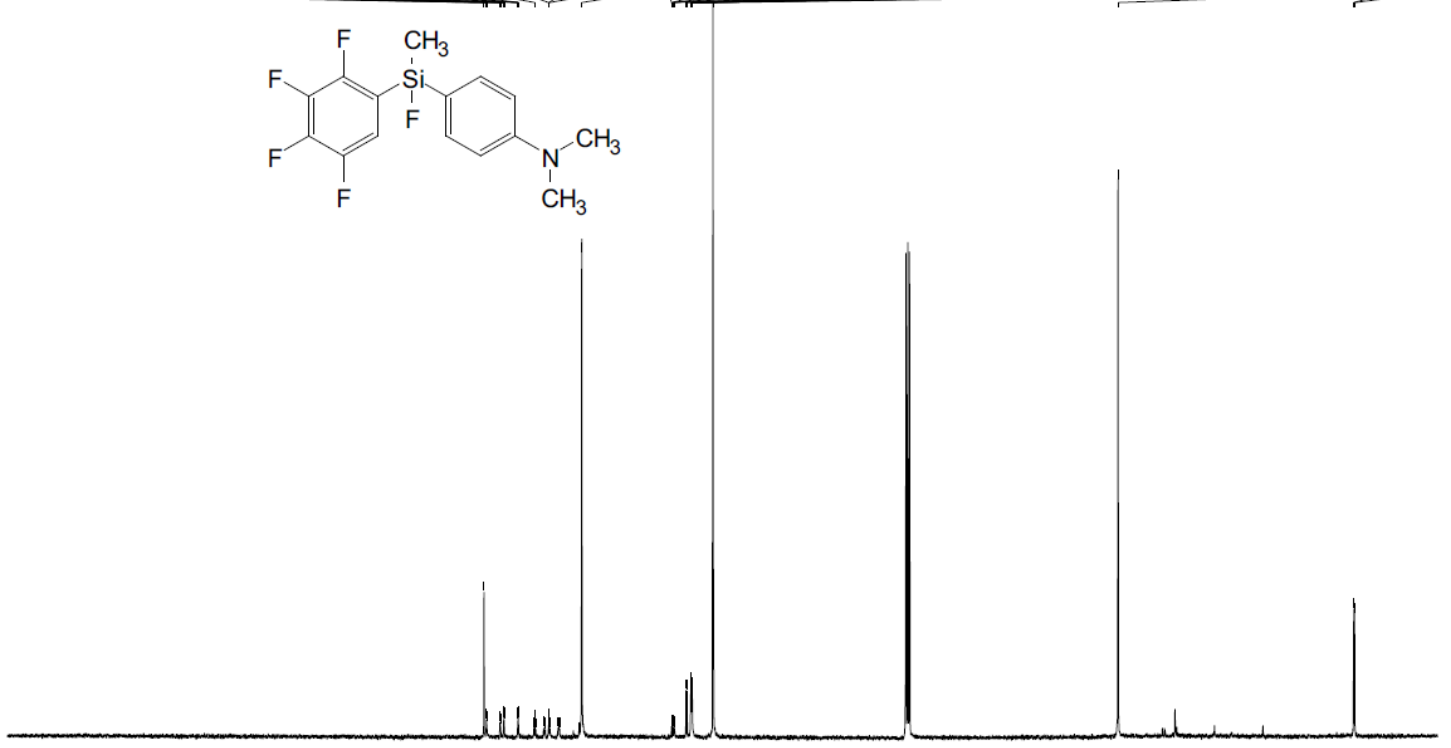

$\begin{array}{lllllllllllllllllllllllll}230 & 220 & 210 & 200 & 190 & 180 & 170 & 160 & 150 & 140 & 130 & 120 & 110 & 100 & 90 & 80 & 70 & 60 & 50 & 40 & 30 & 20 & 10 & 0 & -10\end{array}$ f1 (ppm)

${ }^{13} \mathrm{C} \mathrm{NMR}\left(101 \mathrm{MHz}, \mathrm{CDCl}_{3}\right)$ of $\mathbf{3 j}$ 

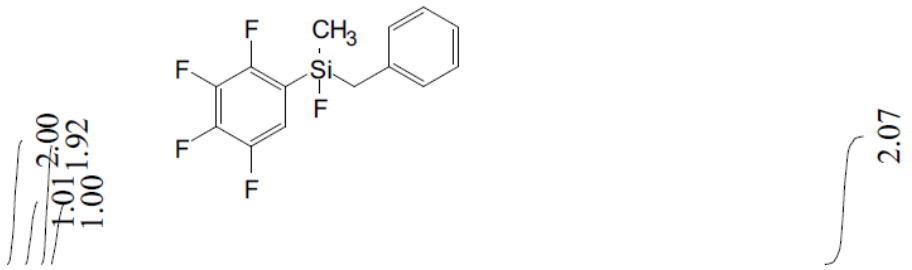

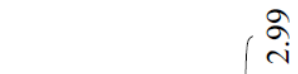

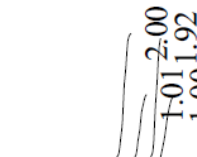

$\int^{\hat{a}}$

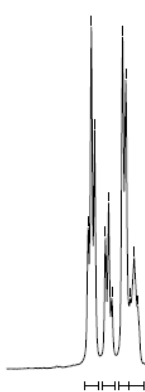

(n)

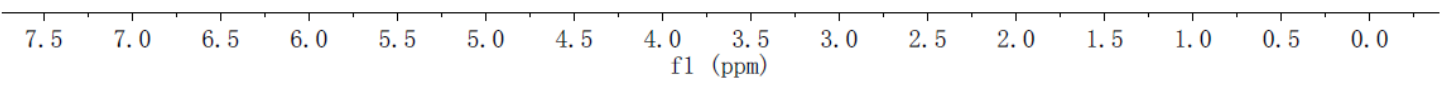

${ }^{1} \mathrm{H}$ NMR $\left(400 \mathrm{MHz}, \mathrm{CDCl}_{3}\right)$ of $\mathbf{3 k}$

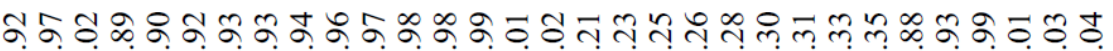

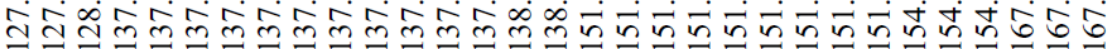<smiles>Cc1cccc(C[Si](F)(F)c2cc(F)c(F)c(F)c2F)c1</smiles>
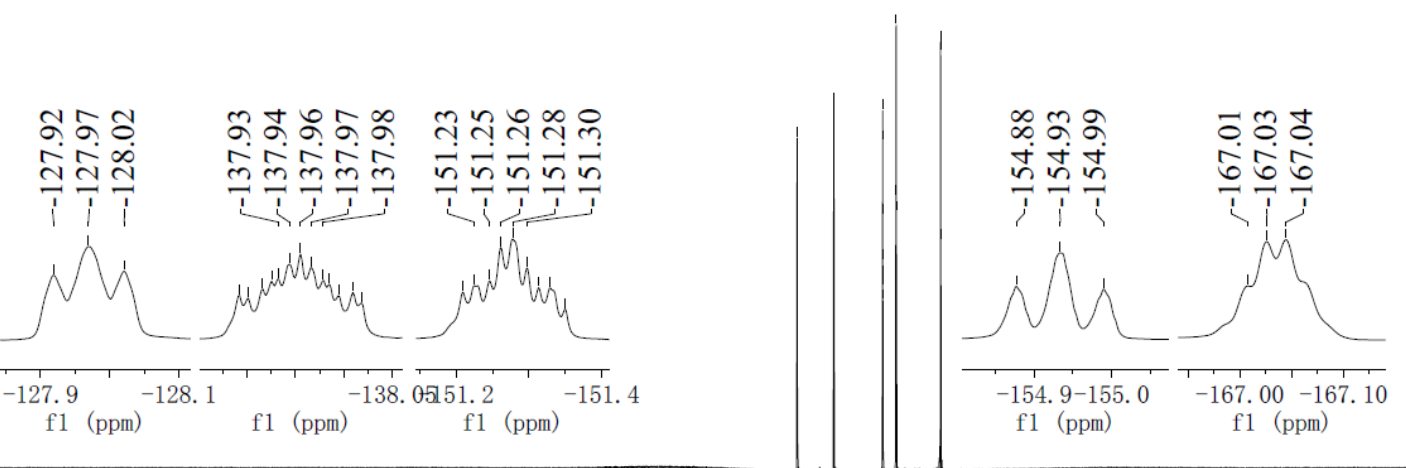

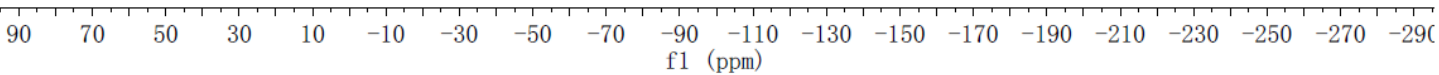

$\left.{ }^{19} \mathrm{~F} \mathrm{NMR} \mathrm{(376} \mathrm{MHz,} \mathrm{CDCl}_{3}\right)$ of $\mathbf{3 k}$ 


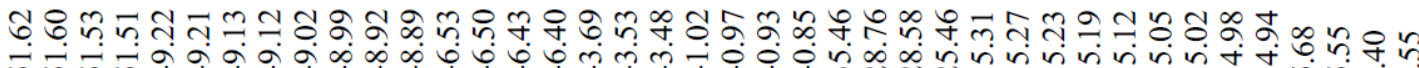

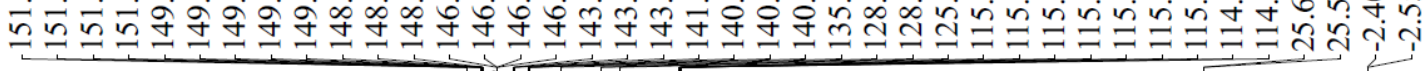

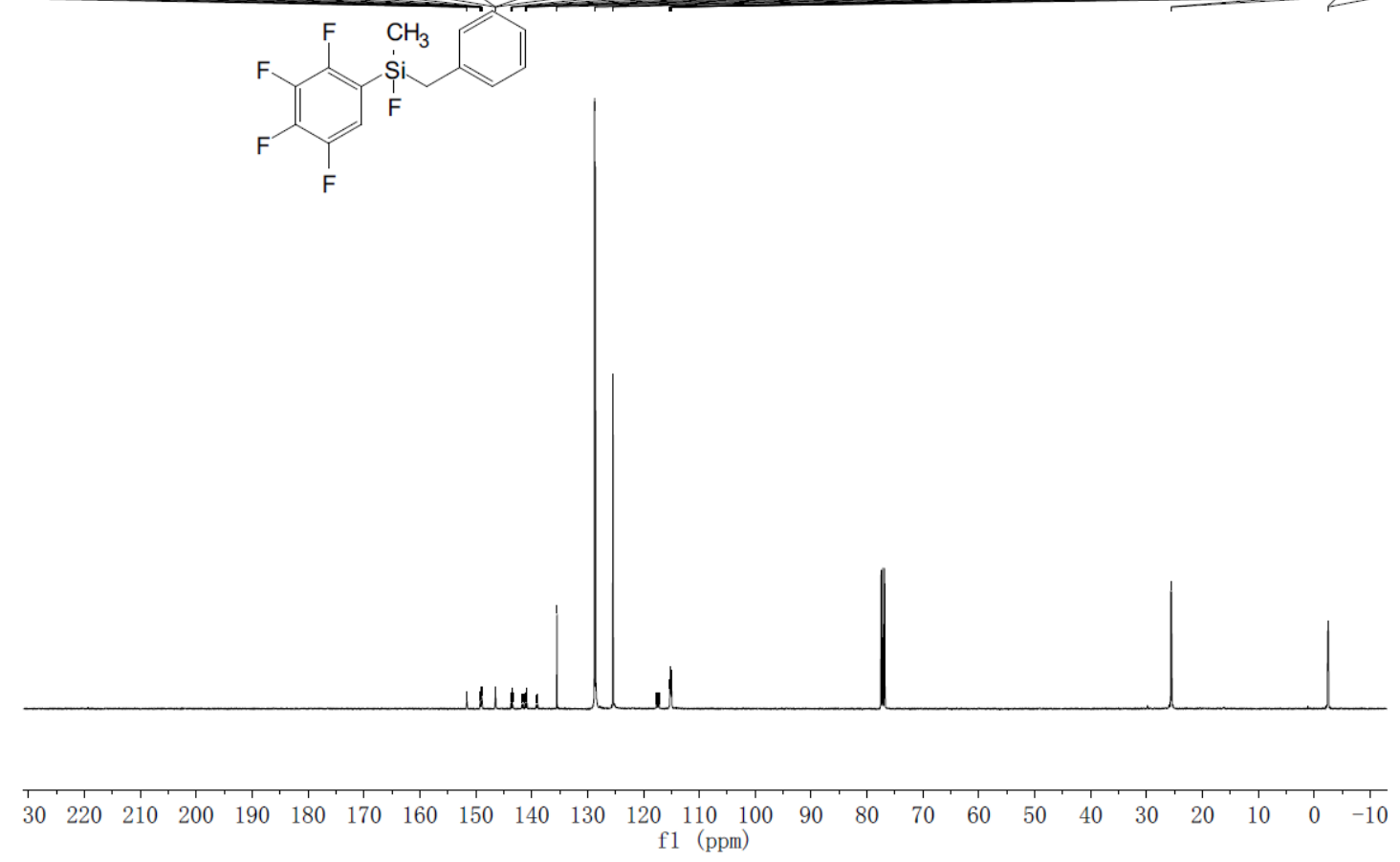

${ }^{13} \mathrm{C} \mathrm{NMR}\left(101 \mathrm{MHz}, \mathrm{CDCl}_{3}\right)$ of $\mathbf{3 k}$ 
<smiles>C[Si](F)(CCc1ccccc1)c1cc(F)c(F)c(F)c1F</smiles>

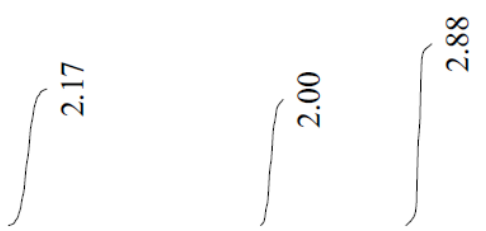

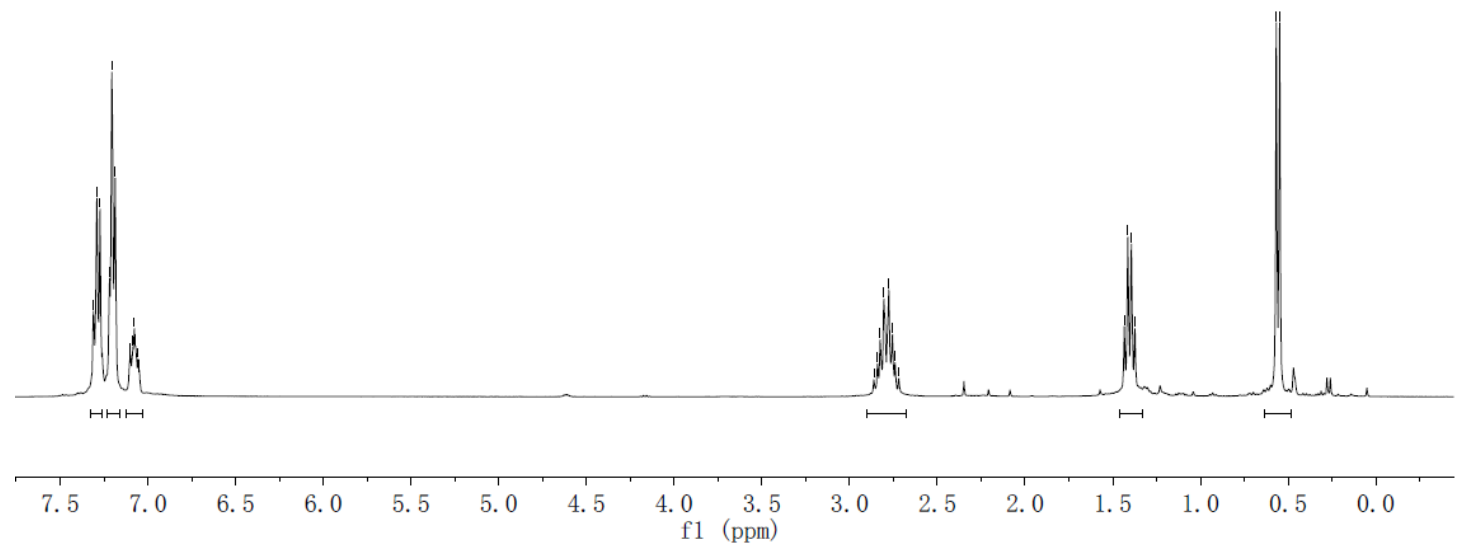

${ }^{1} \mathrm{H} \mathrm{NMR}\left(400 \mathrm{MHz}, \mathrm{CDCl}_{3}\right)$ of $\mathbf{3 l}$

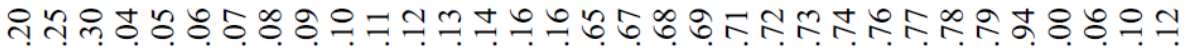

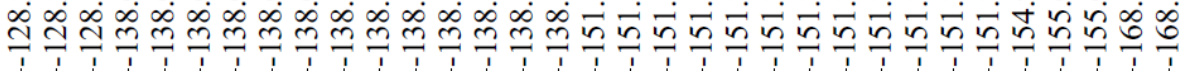<smiles>C[Si](F)(CCc1ccccc1)c1cc(F)c(F)c(F)c1F</smiles>

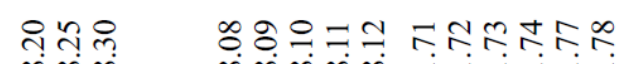

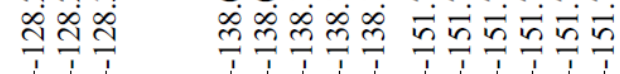
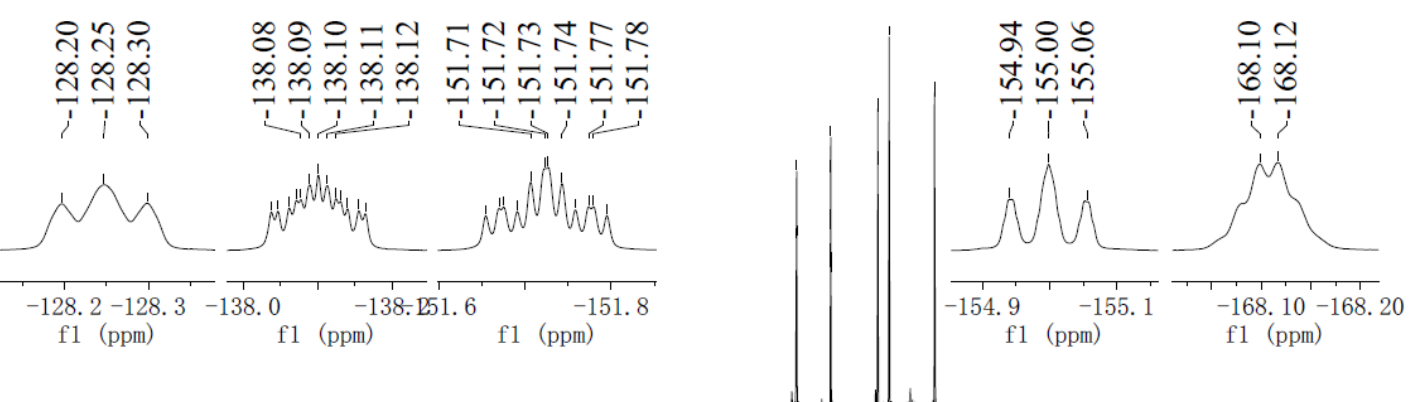

$\begin{array}{lllllllllllllllllllllll}100 & 80 & 60 & 40 & 20 & 0 & -20 & -40 & -60 & -80 & -100 & -120 & -140 & -160 & -180 & -200 & -220 & -240 & -260 & -280 & -300 \\ \mathrm{f} 1 & (\mathrm{pmm}) & & \end{array}$ $\left.{ }^{19} \mathrm{~F} \mathrm{NMR} \mathrm{(376} \mathrm{MHz,} \mathrm{CDCl}_{3}\right)$ of $\mathbf{3 l}$ 


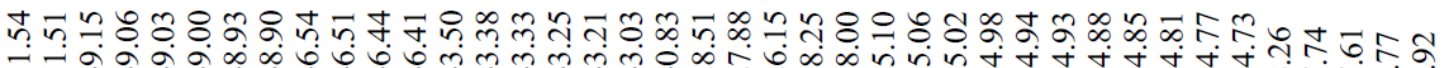

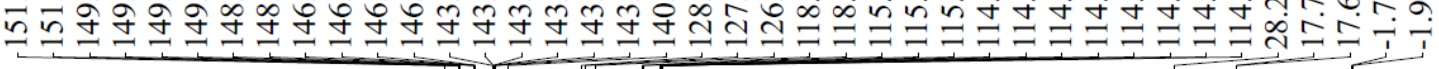<smiles>C[Si](F)(CCc1ccccc1)c1cc(F)c(F)c(F)c1F</smiles>

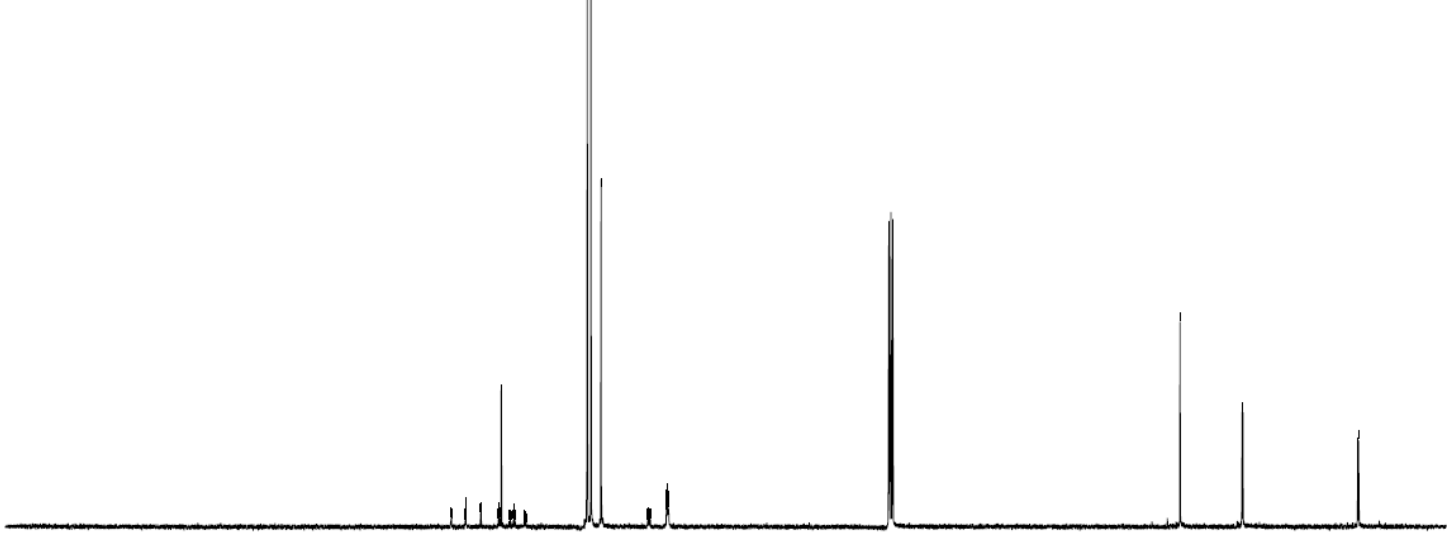

$\begin{array}{lllllllllllll}220 & 210 & 200 & 190 & 180 & 170 & 160 & 150 & 140 & 130 & 120 & 110 & 100 \\ \mathrm{f} 1 & (\mathrm{ppm})\end{array}$

${ }^{13} \mathrm{C}$ NMR $\left(101 \mathrm{MHz}, \mathrm{CDCl}_{3}\right)$ of $\mathbf{3}$ I 
<smiles>CCC[Si](C)(F)c1cc(F)c(F)c(F)c1F</smiles>

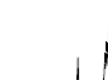

\section{H}

$\mapsto$<smiles>CCC[Si](C)(F)C1=C(F)C(F)C(F)C(F)C1</smiles>

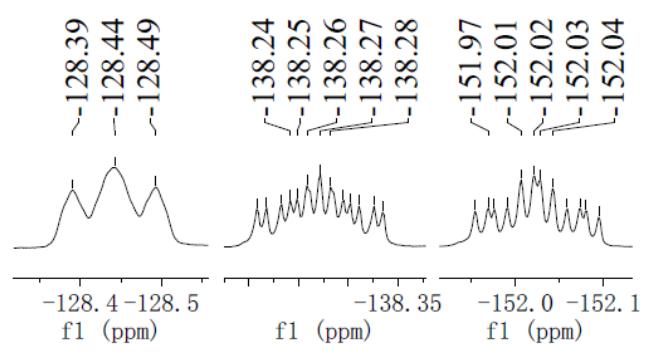

$00 \overline{1}$ ำ

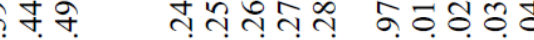

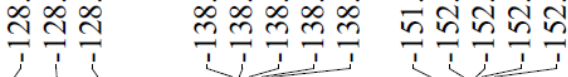

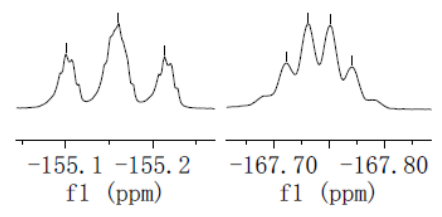

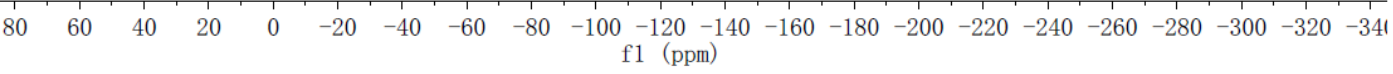

${ }^{19} \mathrm{~F} \mathrm{NMR}\left(376 \mathrm{MHz}, \mathrm{CDCl}_{3}\right)$ of $\mathbf{3 m}$ 


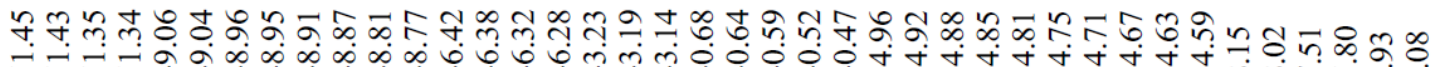

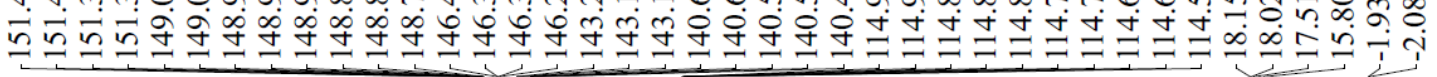<smiles>CCC[Si](C)(F)C1=C(F)C(F)C(F)C(F)C1</smiles>

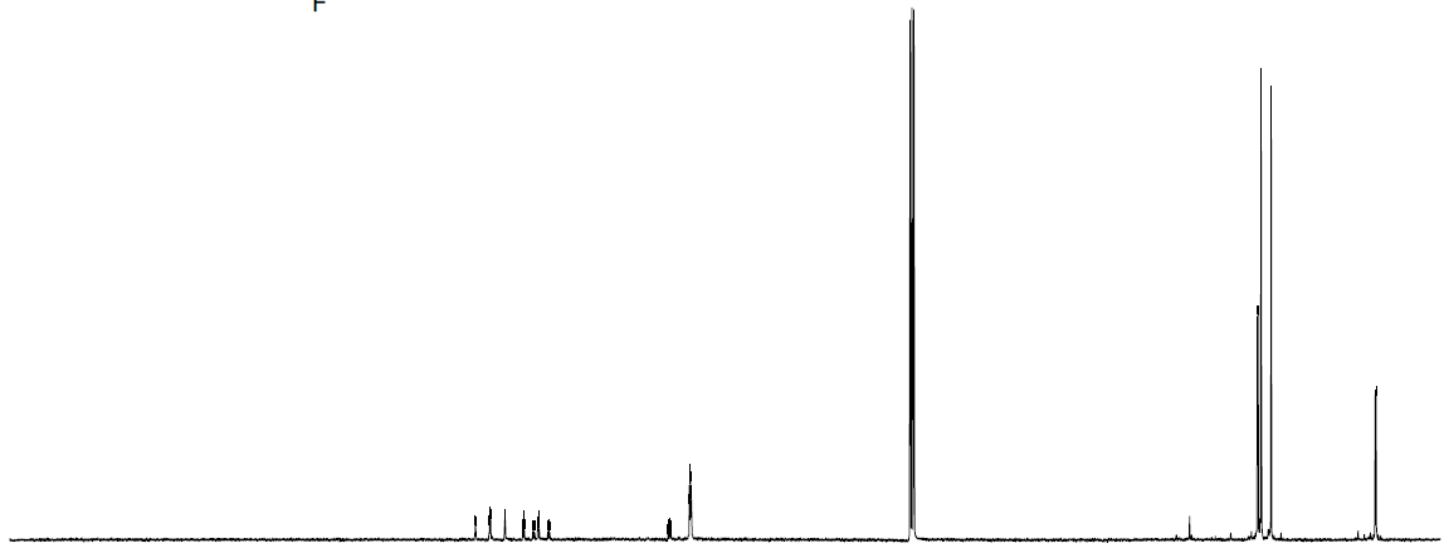

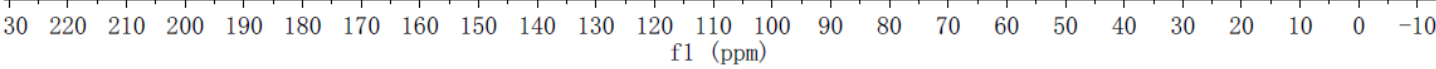
${ }^{13} \mathrm{C} \mathrm{NMR}\left(101 \mathrm{MHz}, \mathrm{CDCl}_{3}\right)$ of $3 \mathrm{~m}$ 


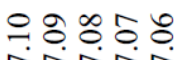

rifir

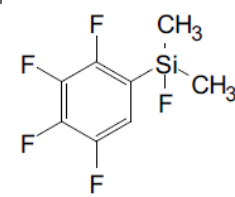

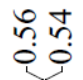

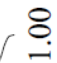

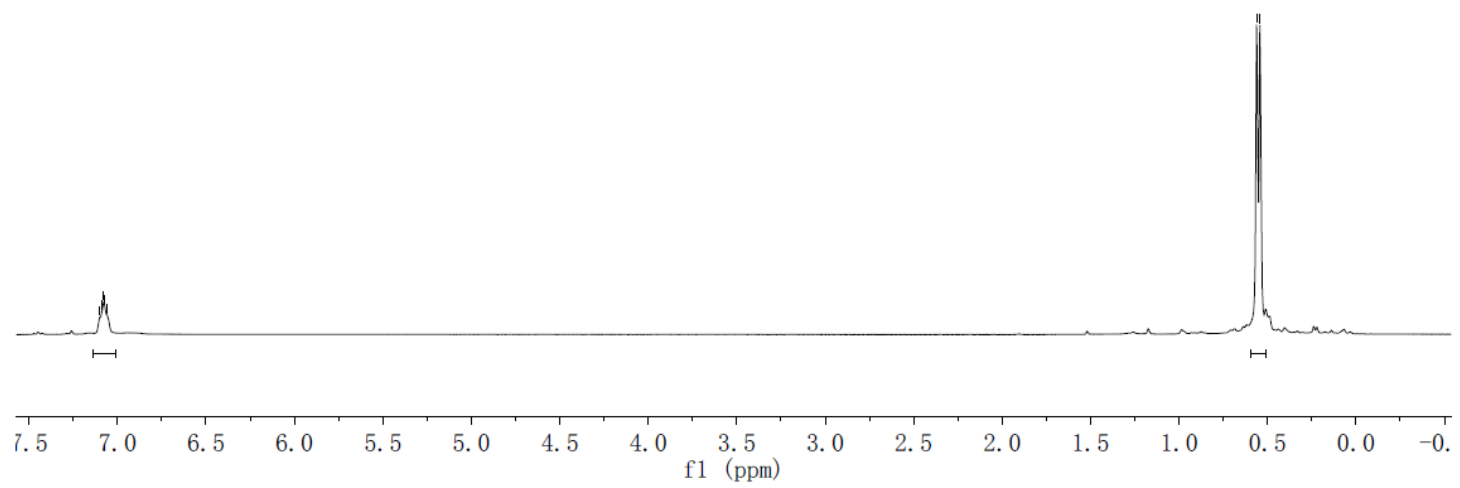

${ }^{1} \mathrm{H} \mathrm{NMR}\left(400 \mathrm{MHz}, \mathrm{CDCl}_{3}\right)$ of $\mathbf{3 n}$

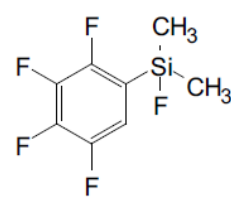

$\bar{\infty}$ 우루

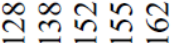

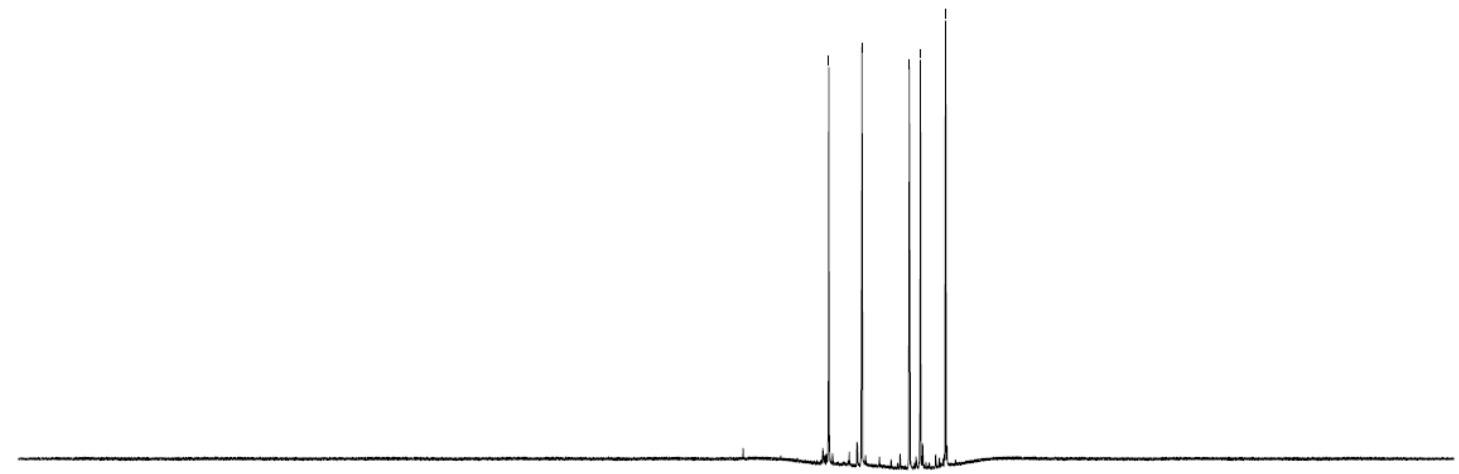

$\begin{array}{llllllllllllllllllllllllllllll}100 & 80 & 60 & 40 & 20 & 0 & -20 & -40 & -60 & -80 & -100 & -120 & -140 & -160 & -180 & -200 & -220 & -240 & -260 & -280 & -300\end{array}$ ${ }^{19} \mathrm{~F} \mathrm{NMR}\left(376 \mathrm{MHz}, \mathrm{CDCl}_{3}\right)$ of $\mathbf{3 n}$ 
<smiles>C[Si](C)(F)c1cc(F)c(F)c(F)c1F</smiles>

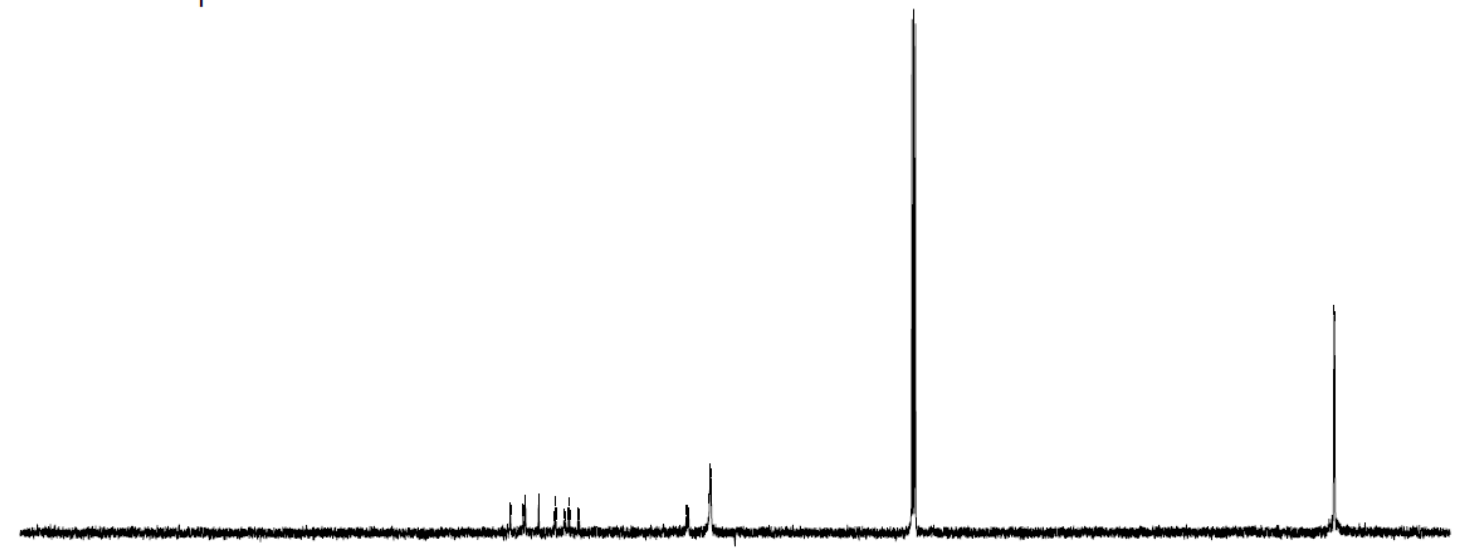

$\begin{array}{lllllllllllllllllllllllllll}10 & 230 & 220 & 210 & 200 & 190 & 180 & 170 & 160 & 150 & 140 & 130 & 120 & 110 & 100 & 90 & 80 & 70 & 60 & 50 & 40 & 30 & 20 & 10 & 0 & -10 & -2(1\end{array}$ f1 (ppm)

$\left.{ }^{13} \mathrm{C} \mathrm{NMR} \mathrm{(101} \mathrm{MHz,} \mathrm{CDCl}_{3}\right)$ of $3 \mathrm{n}$ 

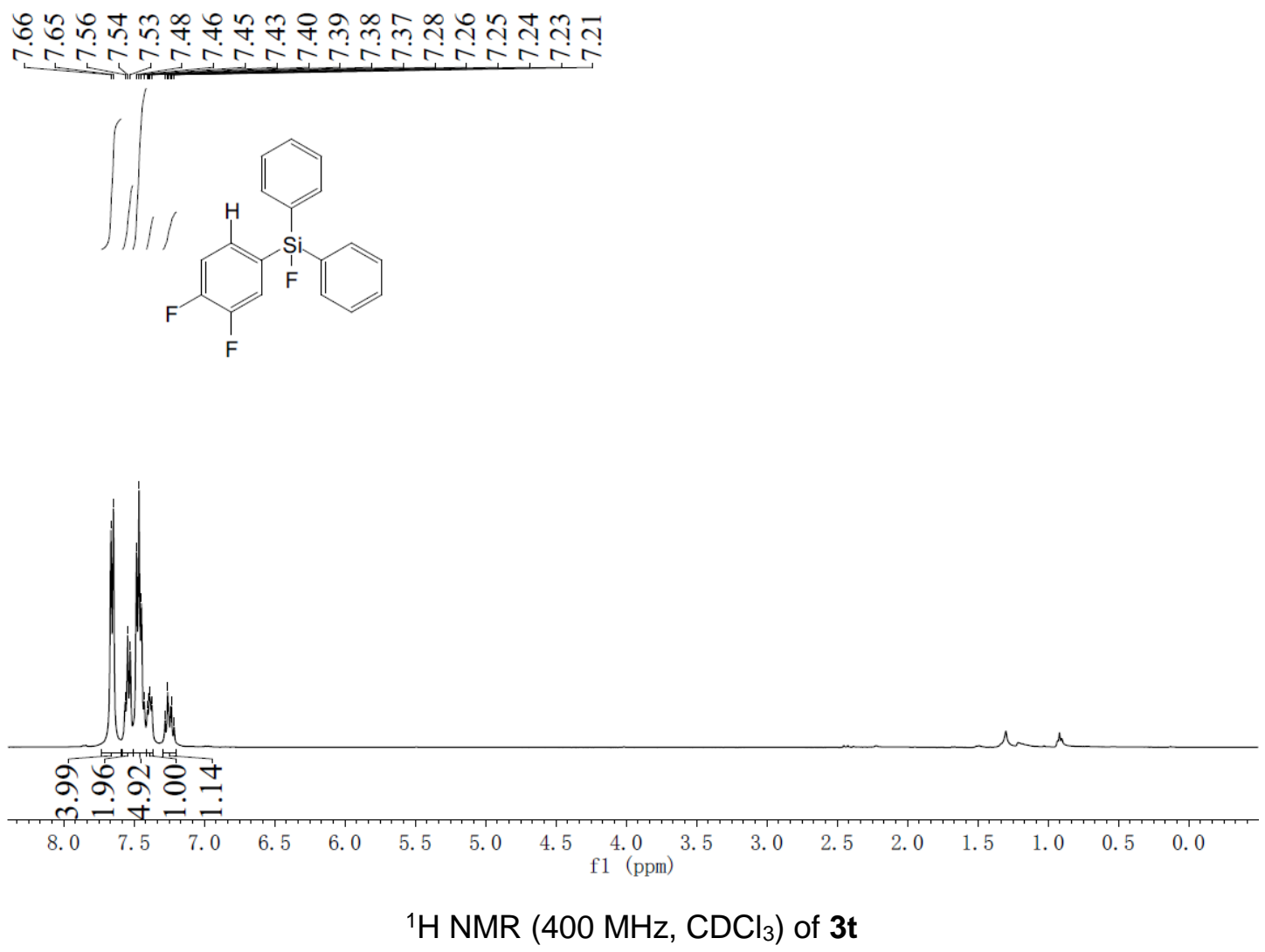

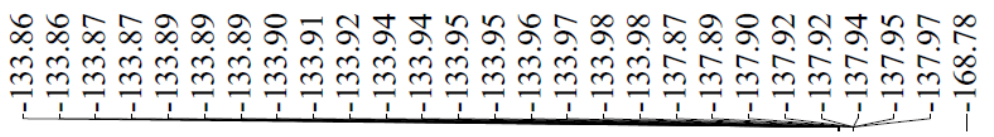
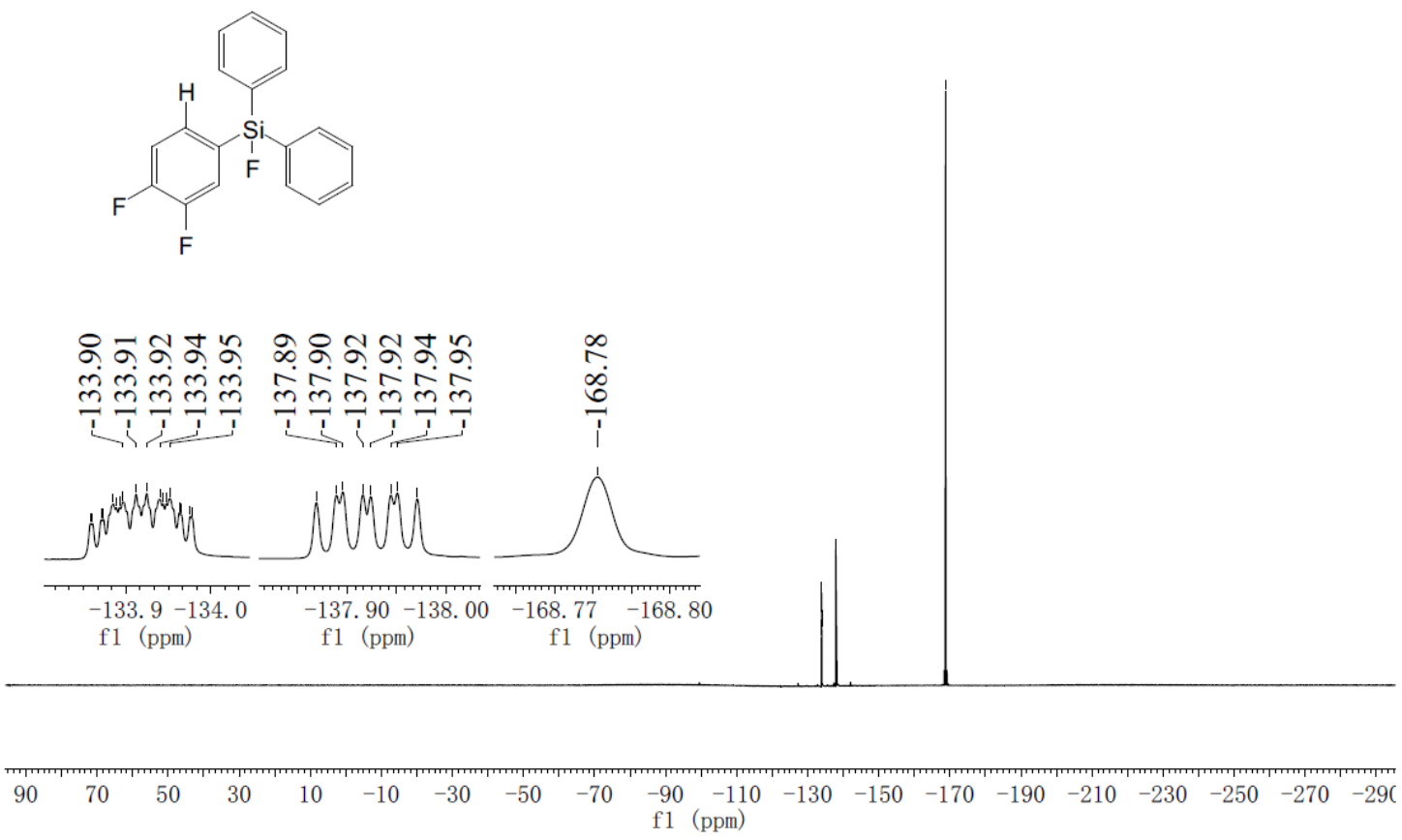

$\left.{ }^{19} \mathrm{~F} \mathrm{NMR} \mathrm{(376} \mathrm{MHz,} \mathrm{CDCl}_{3}\right)$ of $\mathbf{3 t}$

S135 


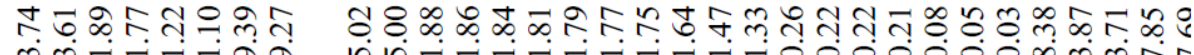

मी

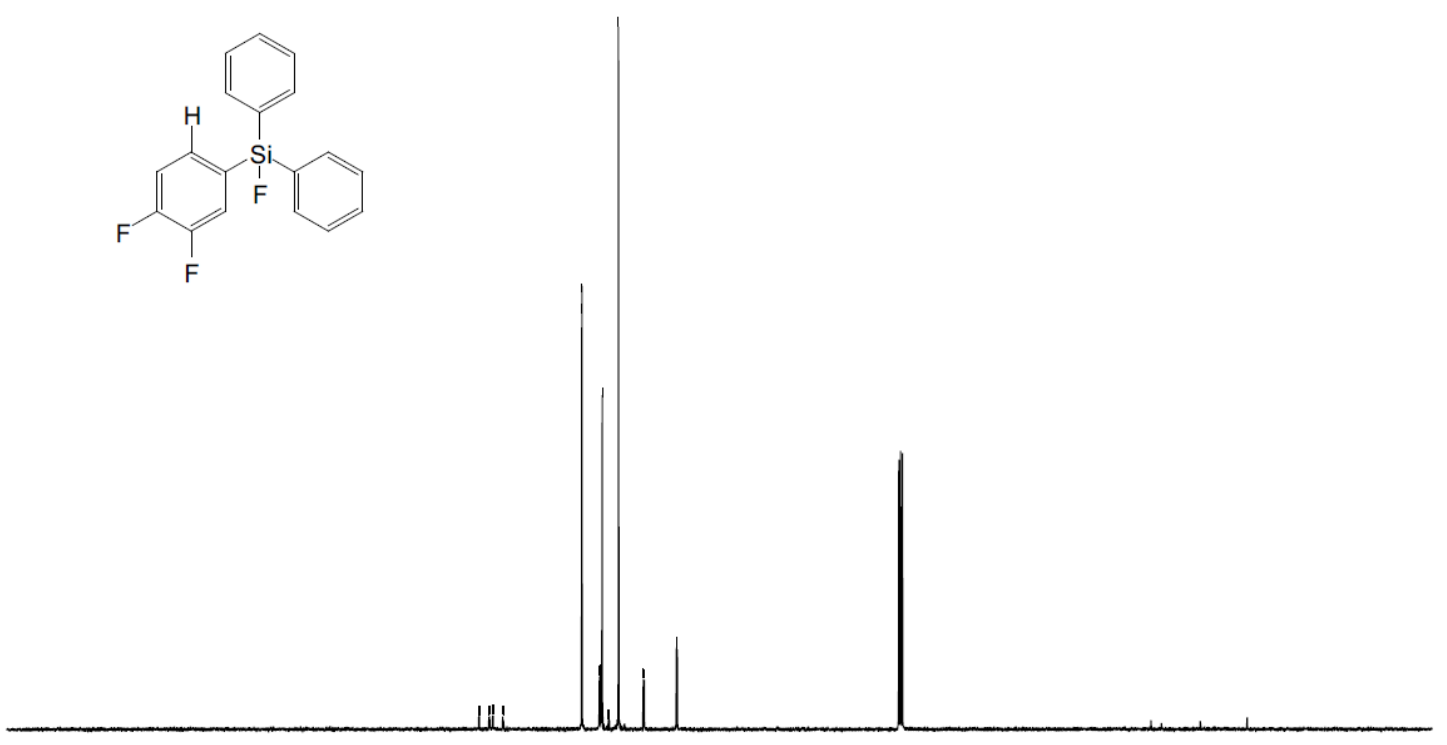

$\begin{array}{lllllllllllllllllllllllll}230 & 220 & 210 & 200 & 190 & 180 & 170 & 160 & 150 & 140 & 130 & 120 & 110 & 100 & 90 & 80 & 70 & 60 & 50 & 40 & 30 & 20 & 10 & 0 & -10\end{array}$ f1 (ppm)

${ }^{13} \mathrm{C} \mathrm{NMR}\left(101 \mathrm{MHz}, \mathrm{CDCl}_{3}\right)$ of $3 \mathrm{t}$ 


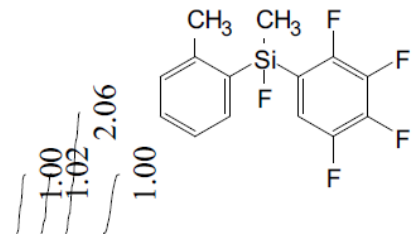

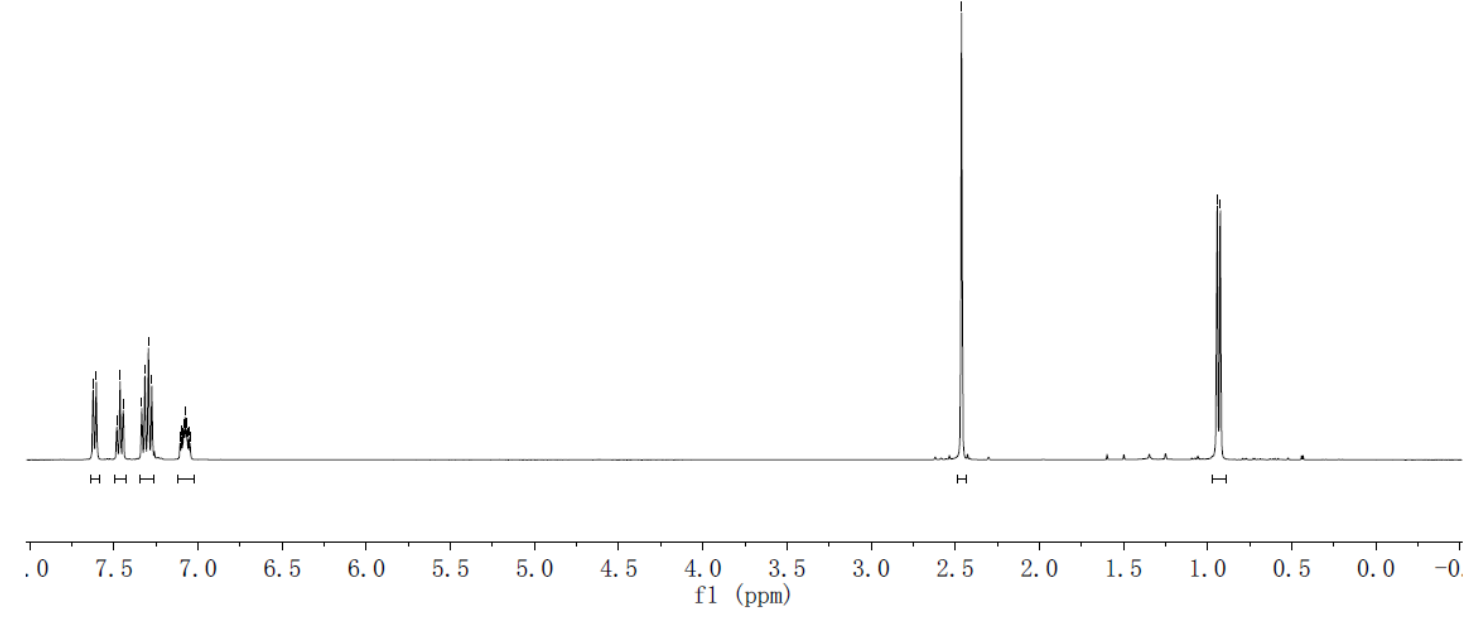

${ }^{1} \mathrm{H}$ NMR $\left(400 \mathrm{MHz}, \mathrm{CDCl}_{3}\right)$ of $6 \mathbf{a}$<smiles>Cc1ccccc1C(C)(C)c1cc(F)c(F)c(F)c1F</smiles>

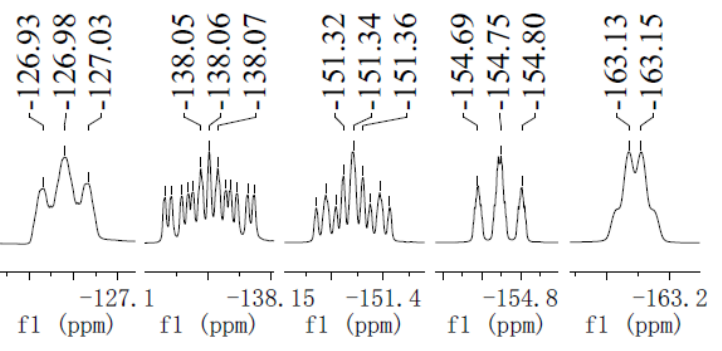

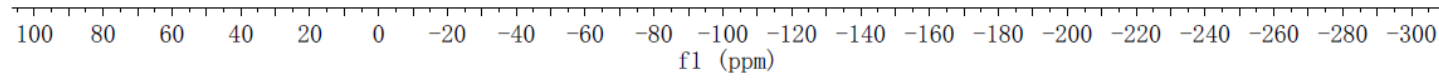
$\left.{ }^{19} \mathrm{~F} \mathrm{NMR} \mathrm{(376} \mathrm{MHz,} \mathrm{CDCl}_{3}\right)$ of $6 \mathrm{a}$ 


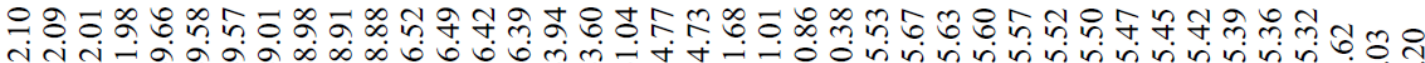
는스는<smiles>Cc1ccccc1C(C)(F)c1cc(F)c(F)c(F)c1F</smiles>

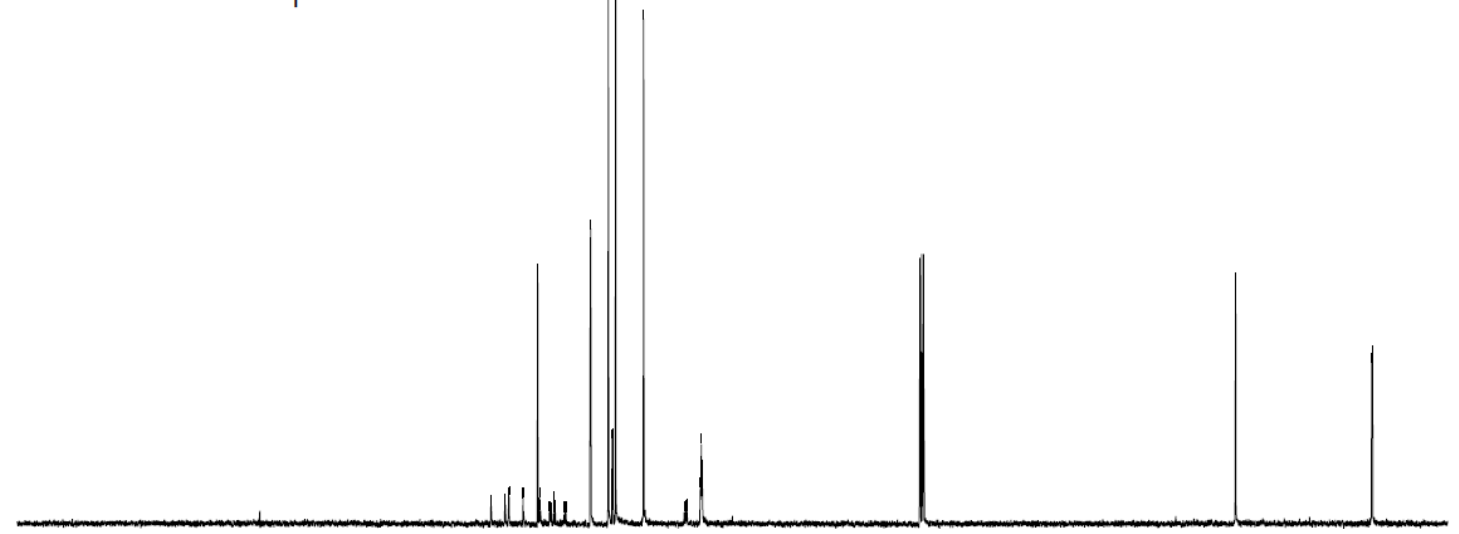

$\begin{array}{lllllllllllllllllllllllllllll}230 & 220 & 210 & 200 & 190 & 180 & 170 & 160 & 150 & 140 & 130 & 120 & 110 & 100 & 90 & 80 & 70 & 60 & 50 & 40 & 30 & 20 & 10 & 0 & -10\end{array}$

${ }^{13} \mathrm{C} \mathrm{NMR}\left(101 \mathrm{MHz}, \mathrm{CDCl}_{3}\right)$ of $\mathbf{6 a}$ 
<smiles>CC[Si](C)(O)c1ccc(-c2ccccc2)cc1</smiles>

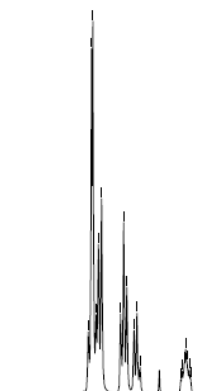
th $\rightarrow$

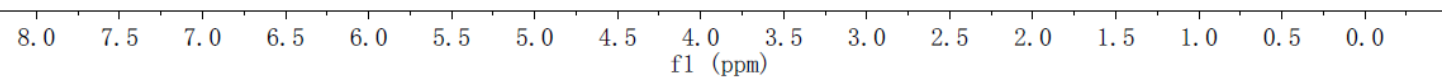

${ }^{1} \mathrm{H} \mathrm{NMR}\left(400 \mathrm{MHz}, \mathrm{CDCl}_{3}\right)$ of $6 \mathbf{b}$

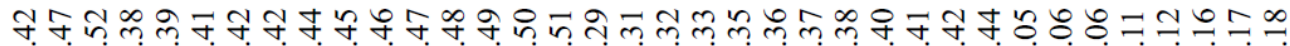

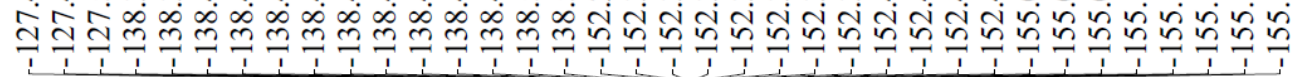<smiles>CSc1ccc(-c2ccccc2)cc1</smiles>

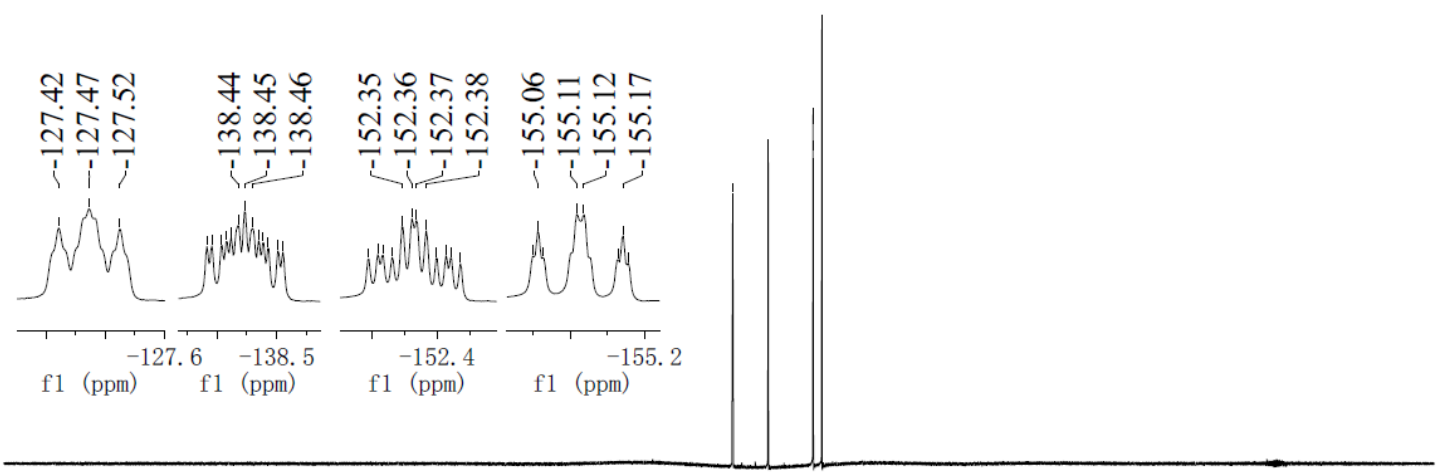

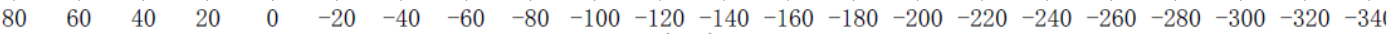
f1 (ppm)

${ }^{19} \mathrm{~F} \mathrm{NMR}\left(376 \mathrm{MHz}, \mathrm{CDCl}_{3}\right)$ of $6 \mathbf{b}$ 


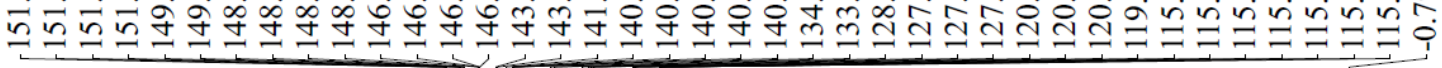

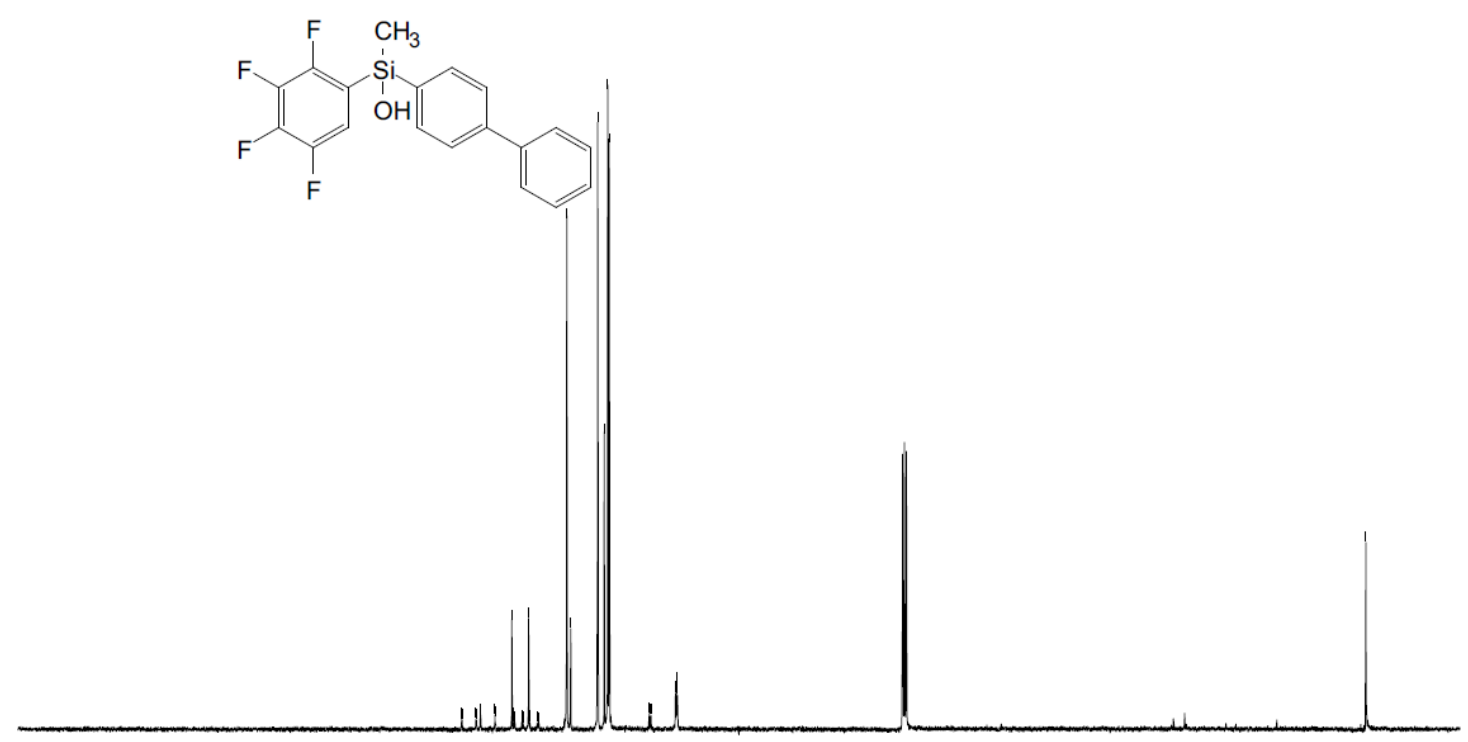

$\begin{array}{lllllllllllllllllllllllll}220 & 210 & 200 & 190 & 180 & 170 & 160 & 150 & 140 & 130 & 120 & 110 & 100 & 90 & 80 & 70 & 60 & 50 & 40 & 30 & 20 & 10 & 0 & -10 \\ \mathrm{f1}(\mathrm{ppm}) & & & \end{array}$

${ }^{13} \mathrm{C} \mathrm{NMR}\left(101 \mathrm{MHz}, \mathrm{CDCl}_{3}\right)$ of $\mathbf{6 b}$ 


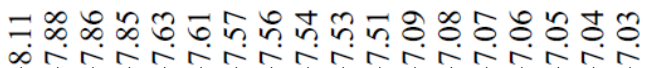

$\stackrel{\infty}{\infty}$
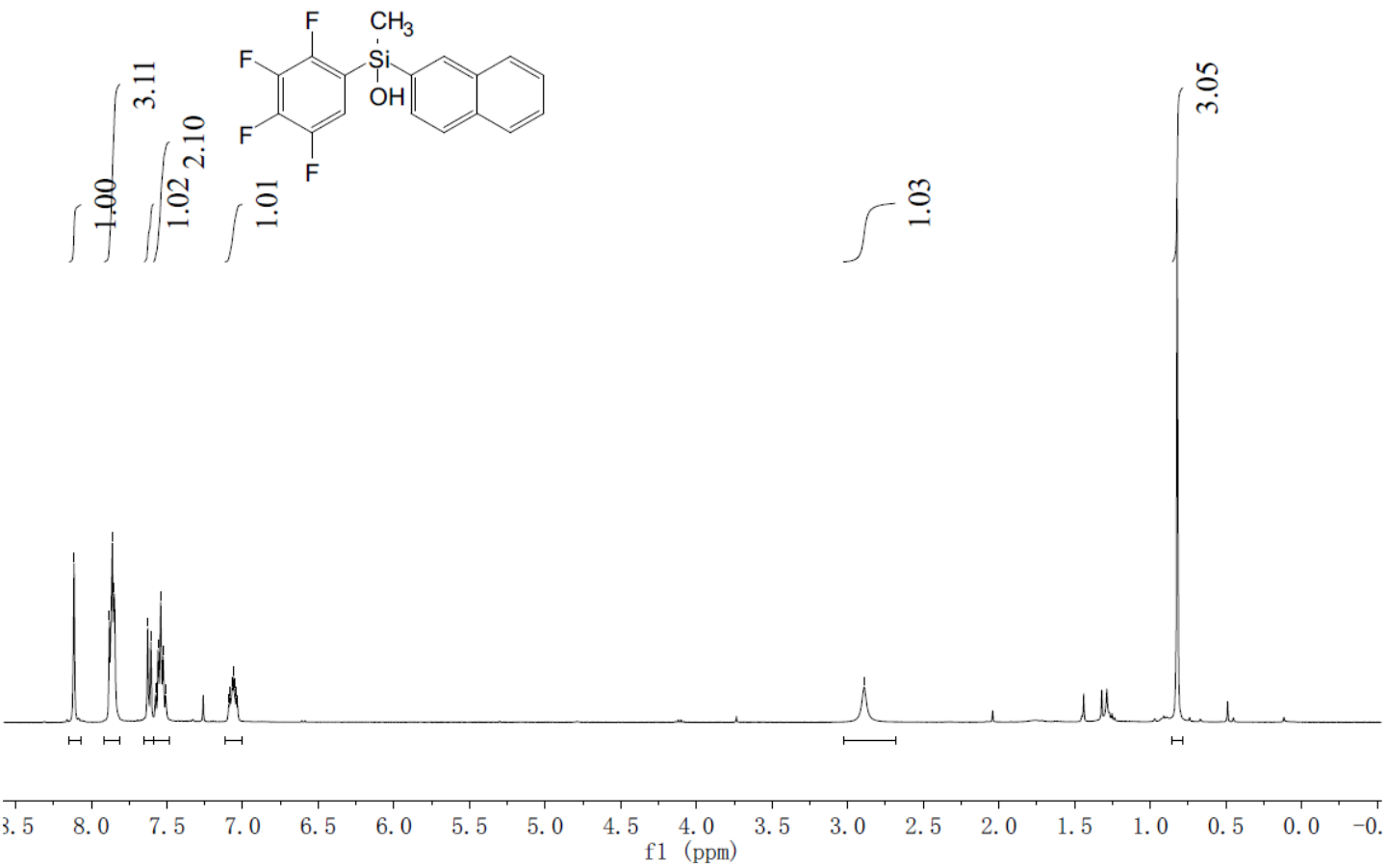

${ }^{1} \mathrm{H} \mathrm{NMR}\left(400 \mathrm{MHz}, \mathrm{CDCl}_{3}\right)$ of $6 \mathrm{c}$

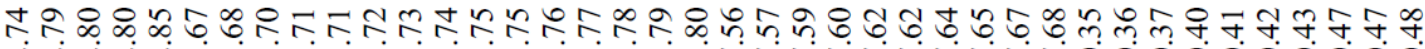

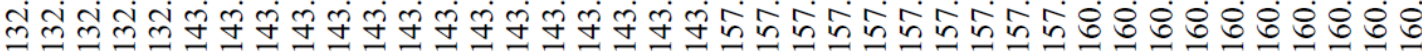<smiles>Cc1cc(OSc2ccc3ccccc3c2)ccc1F</smiles>

용요

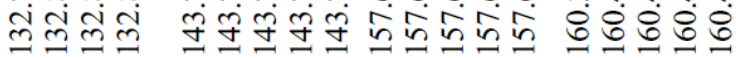

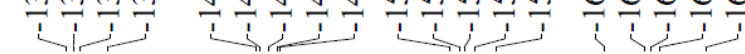

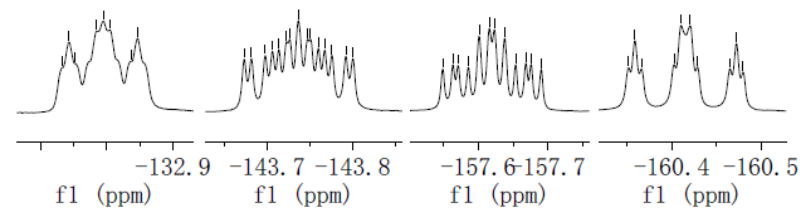

$\begin{array}{lllllllllllllllllllll}90 & 70 & 50 & 30 & 10 & -10 & -30 & -50 & -70 & -90 & -110 & -130 & -150 & -170 & -190 & -210 & -230 & -250 & -270 & -29( \\ & & & \end{array}$ ${ }^{19} \mathrm{~F} \mathrm{NMR}\left(376 \mathrm{MHz}, \mathrm{CDCl}_{3}\right)$ of $6 \mathrm{c}$ 


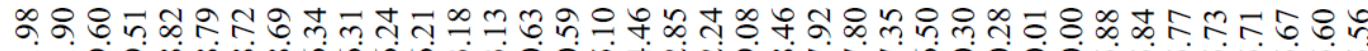

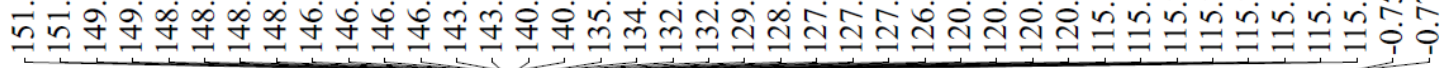

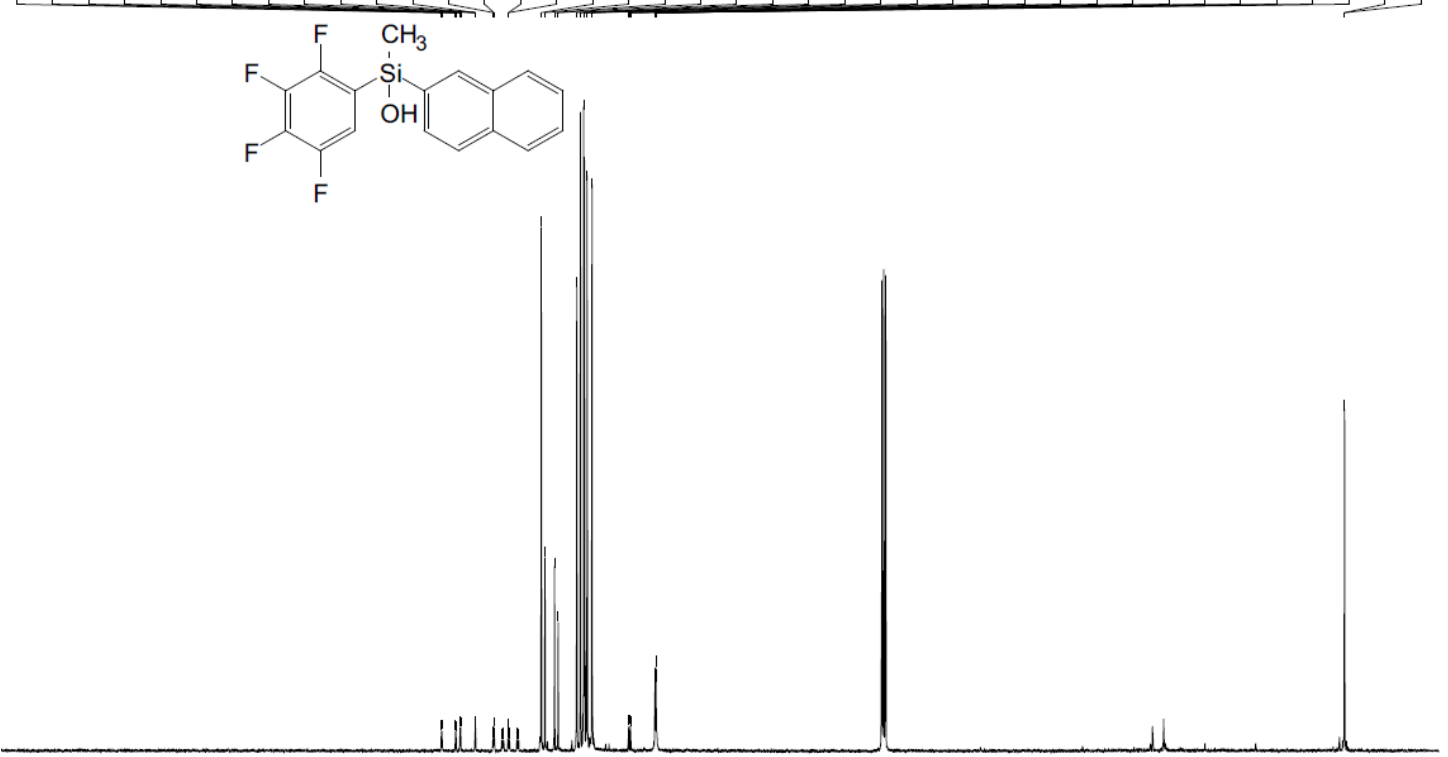

$\begin{array}{llllllllllllllllllllllllll}220 & 210 & 200 & 190 & 180 & 170 & 160 & 150 & 140 & 130 & 120 & 110 & 100 & 90 & 80 & 70 & 60 & 50 & 40 & 30 & 20 & 10 & 0 & -10\end{array}$

${ }^{13} \mathrm{C} \mathrm{NMR}\left(101 \mathrm{MHz}, \mathrm{CDCl}_{3}\right)$ of $6 \mathrm{c}$ 
<smiles>COc1ccc2ccccc2c1OC(C)c1cc(F)c(F)c(F)c1F</smiles><smiles></smiles>
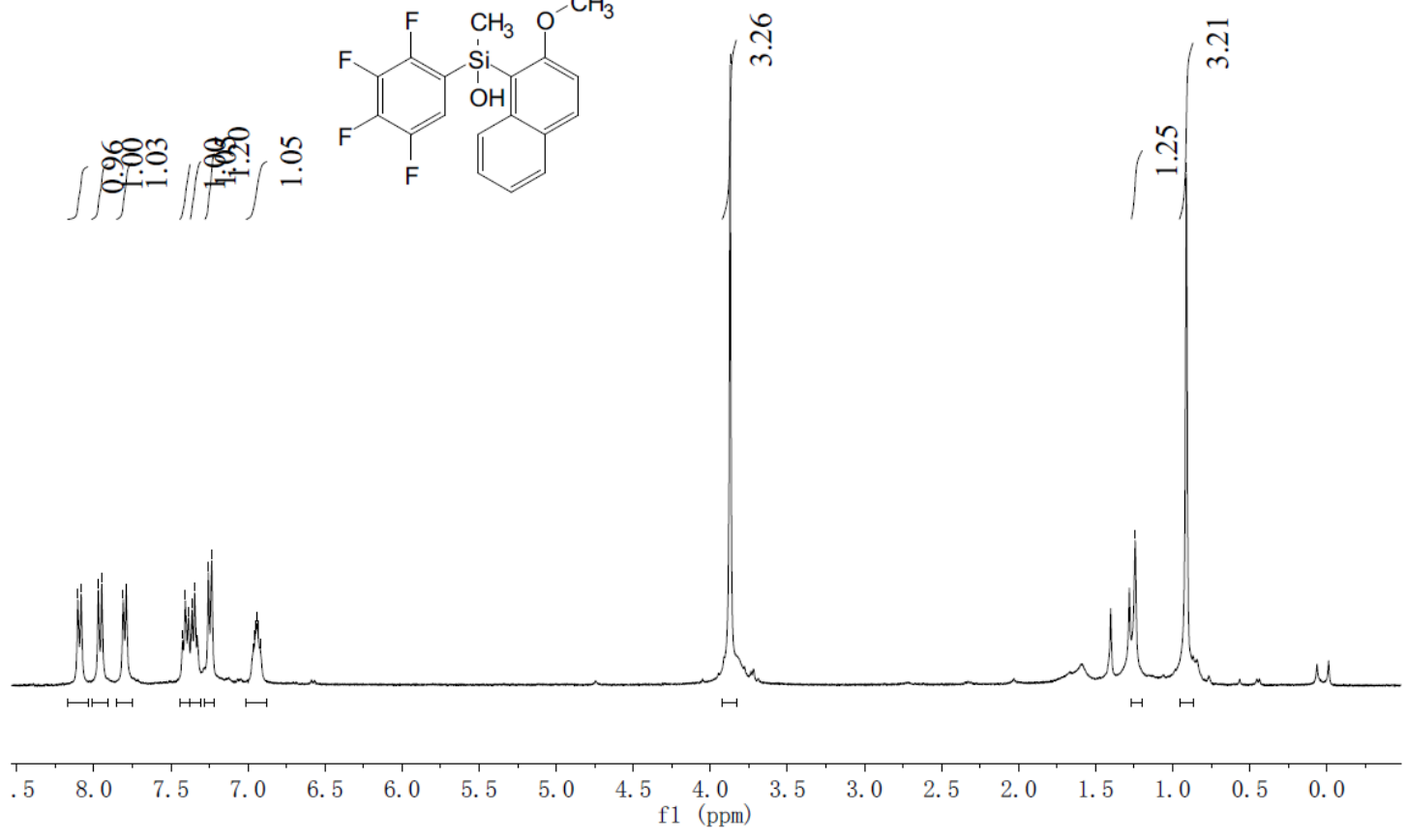

${ }^{1} \mathrm{H} \mathrm{NMR}\left(400 \mathrm{MHz}, \mathrm{CDCl}_{3}\right)$ of $\mathbf{6 d}$

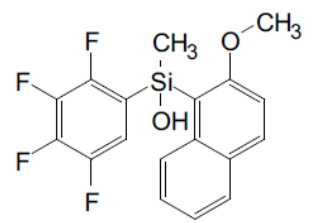

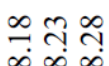

$\stackrel{i}{i} \stackrel{\infty}{i}$

พ⿻ำ

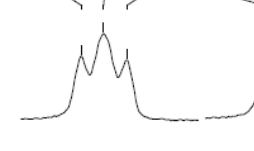



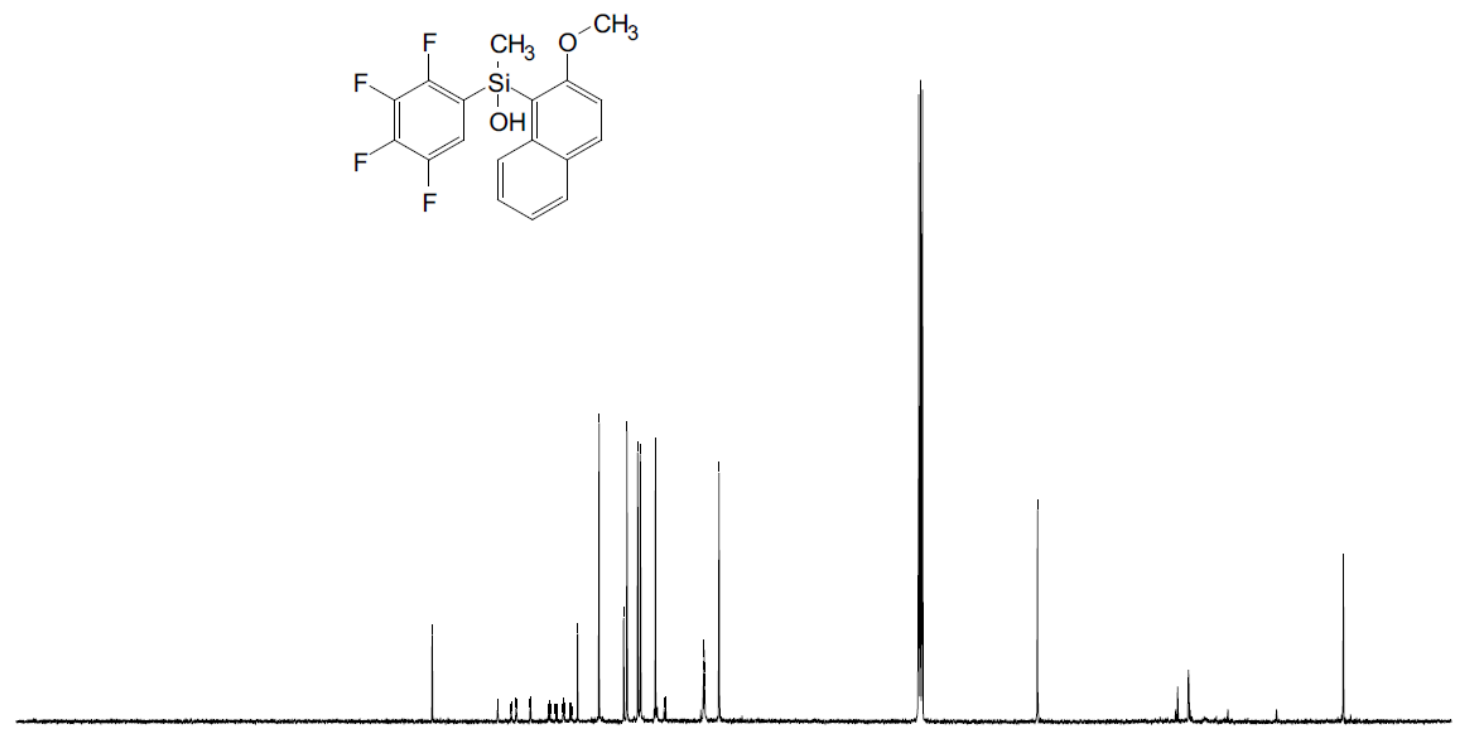

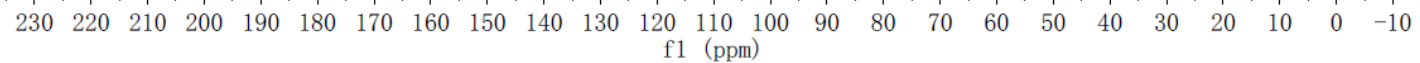

${ }^{13} \mathrm{C} \mathrm{NMR}\left(101 \mathrm{MHz}, \mathrm{CDCl}_{3}\right)$ of $6 \mathrm{~d}$ 
<smiles>C[Si](c1ccc(F)cc1)(c1ccc(-n2cccc2)cc1)c1ccccc1F</smiles>
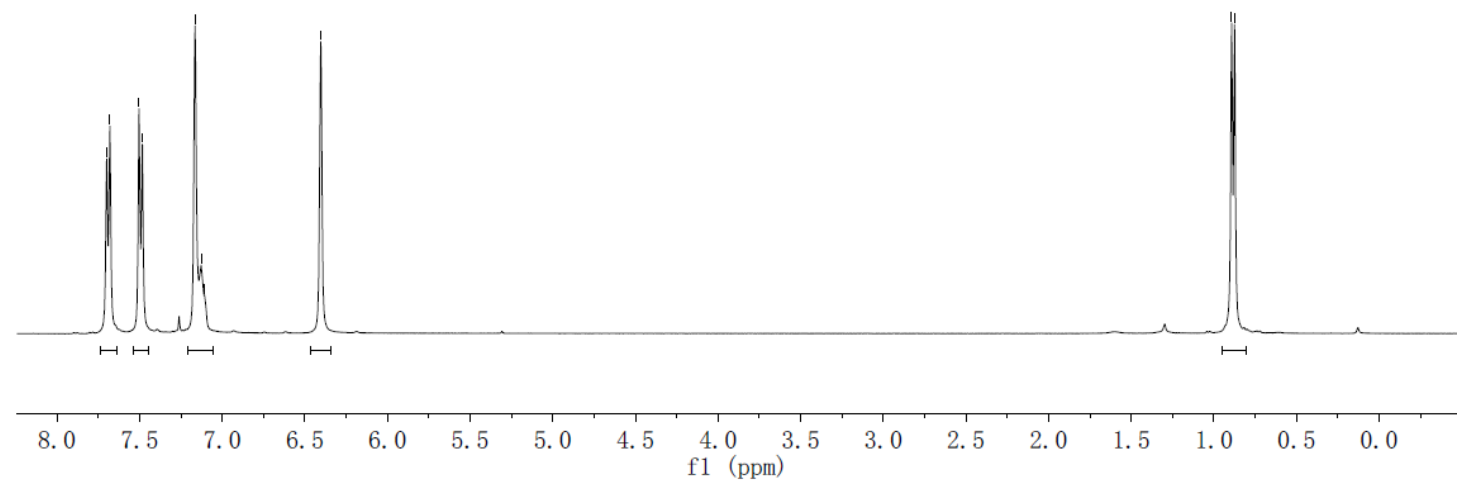

${ }^{1} \mathrm{H} \mathrm{NMR}\left(400 \mathrm{MHz}, \mathrm{CDCl}_{3}\right)$ of $6 \mathrm{e}$

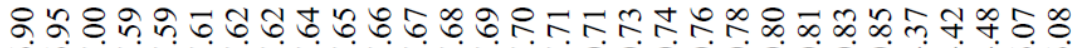

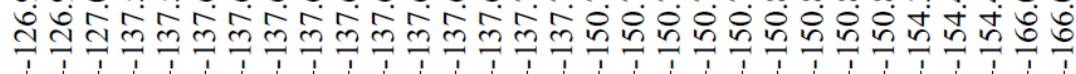<smiles>C[Si](F)(c1ccc(-n2cccc2)cc1)c1cc(F)c(F)c(F)c1F</smiles>

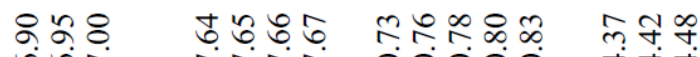
서
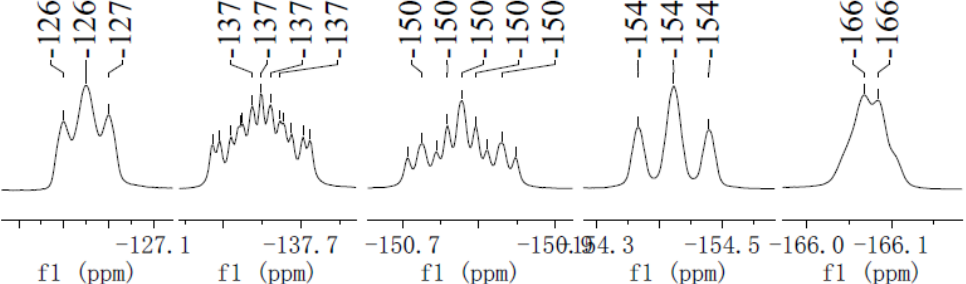

f1 (ppm)

f1 (ppm)

f1 (ppm)

f1 (ppm)

e1 (ppm)

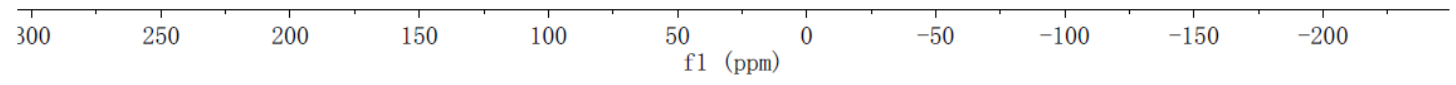

${ }^{19} \mathrm{~F} \mathrm{NMR}\left(376 \mathrm{MHz}, \mathrm{CDCl}_{3}\right)$ of $6 \mathrm{e}$ 


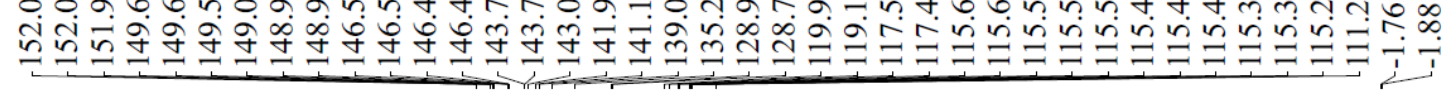

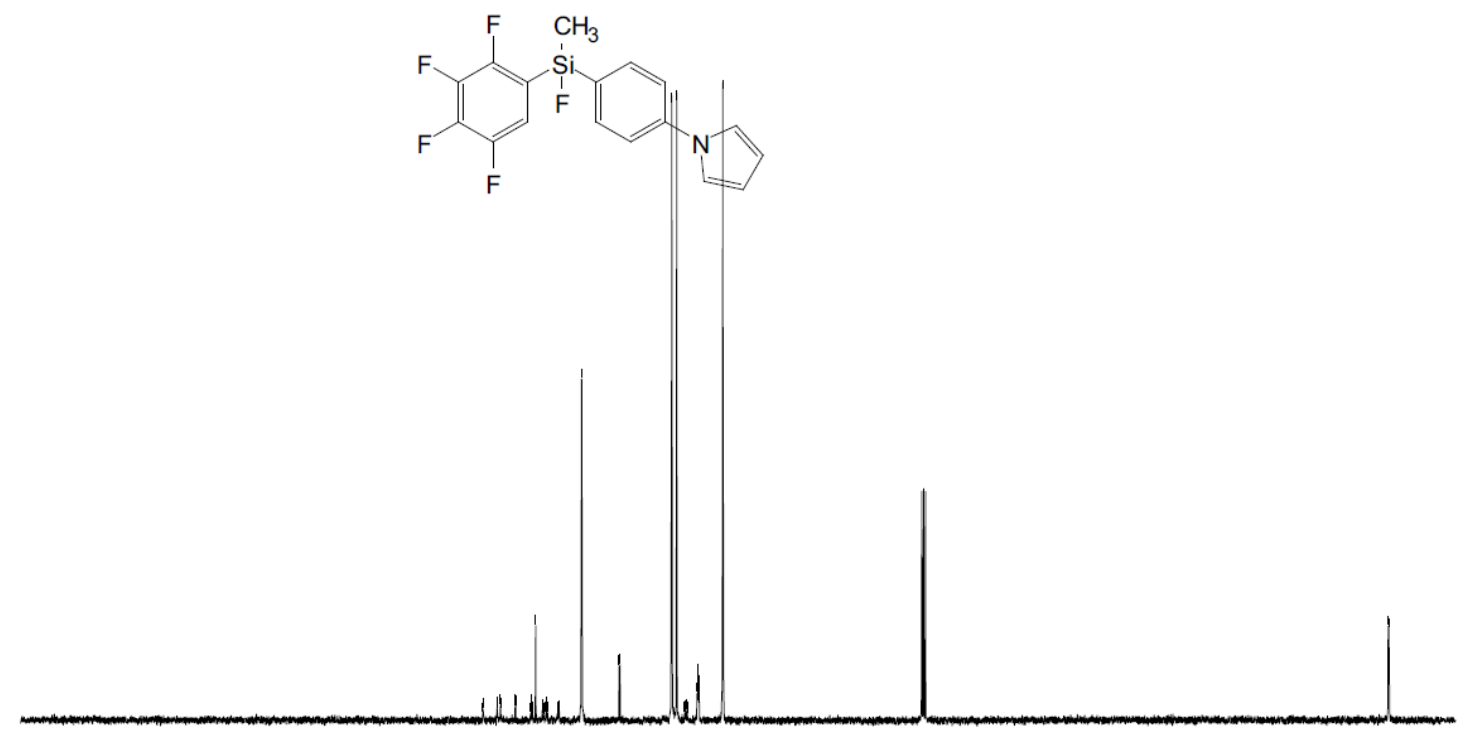

$\begin{array}{lllllllllllllllllllllllllllll}30 & 220 & 210 & 200 & 190 & 180 & 170 & 160 & 150 & 140 & 130 & 120 & 110 & 100 & 90 & 80 & 70 & 60 & 50 & 40 & 30 & 20 & 10 & 0 & -10\end{array}$

${ }^{13} \mathrm{C} \mathrm{NMR}\left(101 \mathrm{MHz}, \mathrm{CDCl}_{3}\right)$ of $6 \mathrm{e}$ 

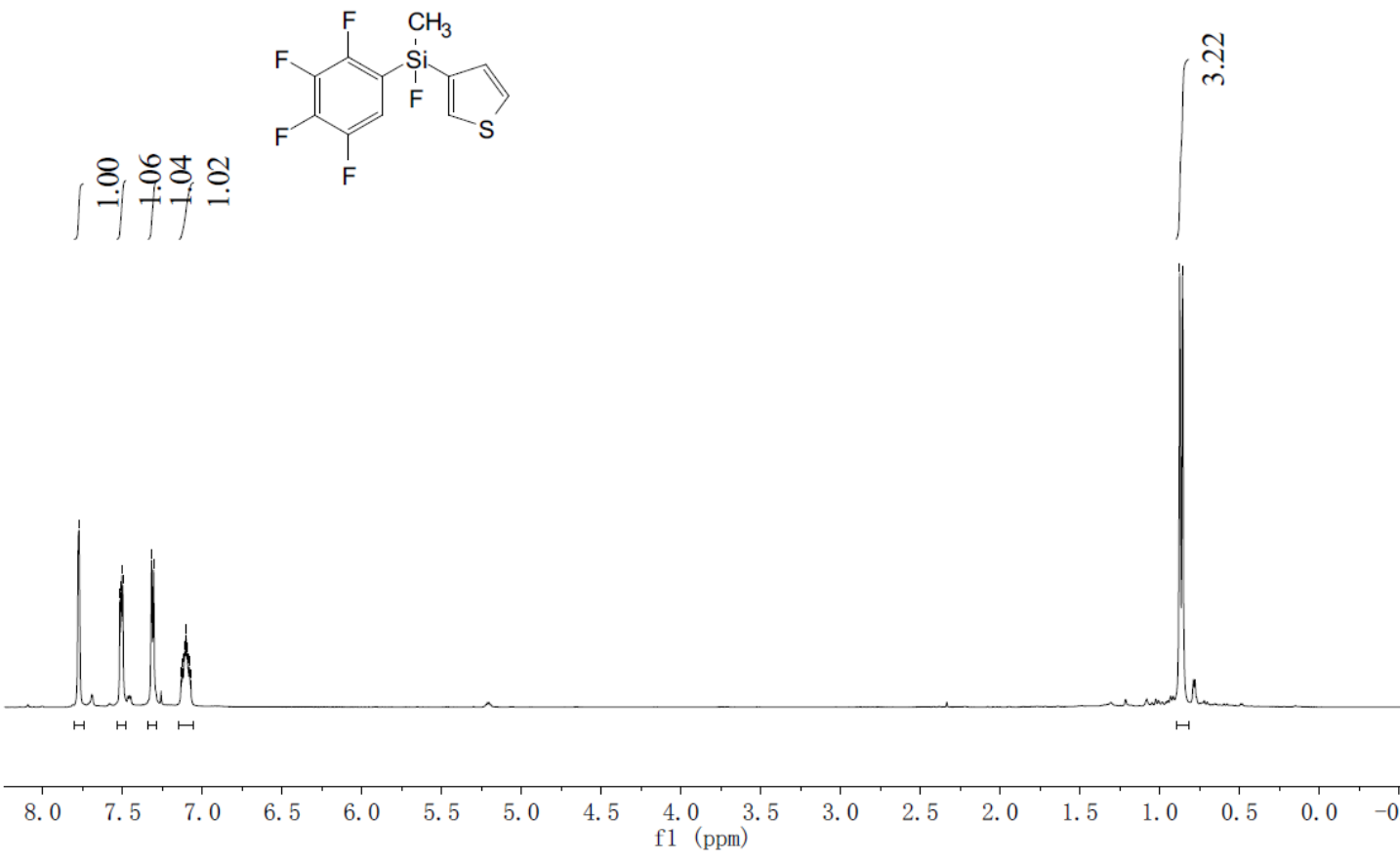

${ }^{1} \mathrm{H} \mathrm{NMR}\left(400 \mathrm{MHz}, \mathrm{CDCl}_{3}\right)$ of $6 \mathrm{f}$

خุ กิ

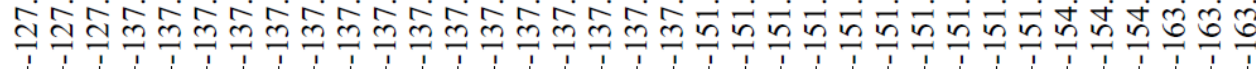<smiles>C[Si](c1ccsc1)(c1ccsc1)c1cc(F)c(F)c(F)c1F</smiles>
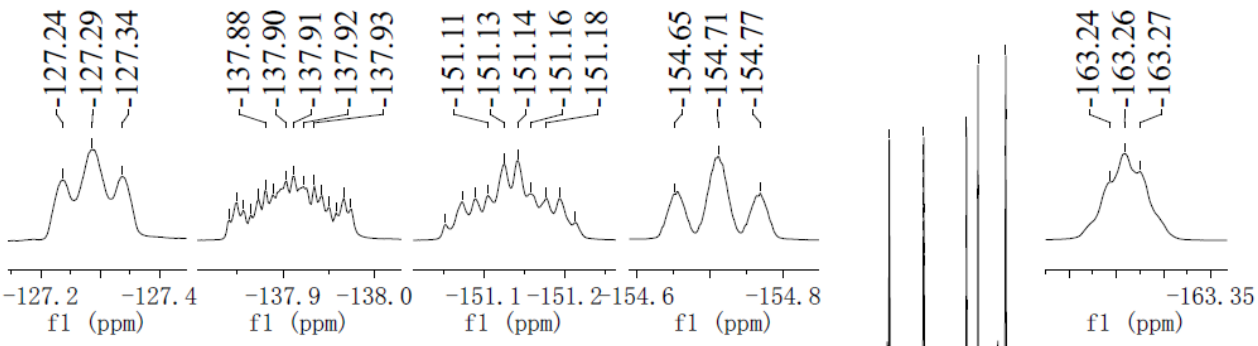

$\begin{array}{rlllllllllllllllllllll}140 & 120 & 100 & 80 & 60 & 40 & 20 & 0 & -20 & -40 & -60 & -80 & -100 & -120 & -140 & -160 & -180 & -200 & -220 & -240 & -260 & -280 \\ \text { f1 } & (\mathrm{ppm})\end{array}$

${ }^{19} \mathrm{~F}$ NMR $\left(376 \mathrm{MHz}, \mathrm{CDCl}_{3}\right)$ of $6 \mathbf{6 f}$ 


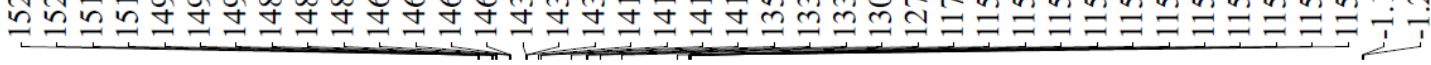<smiles>Cc1ccc(-c2ccsc2)cc1F</smiles> 

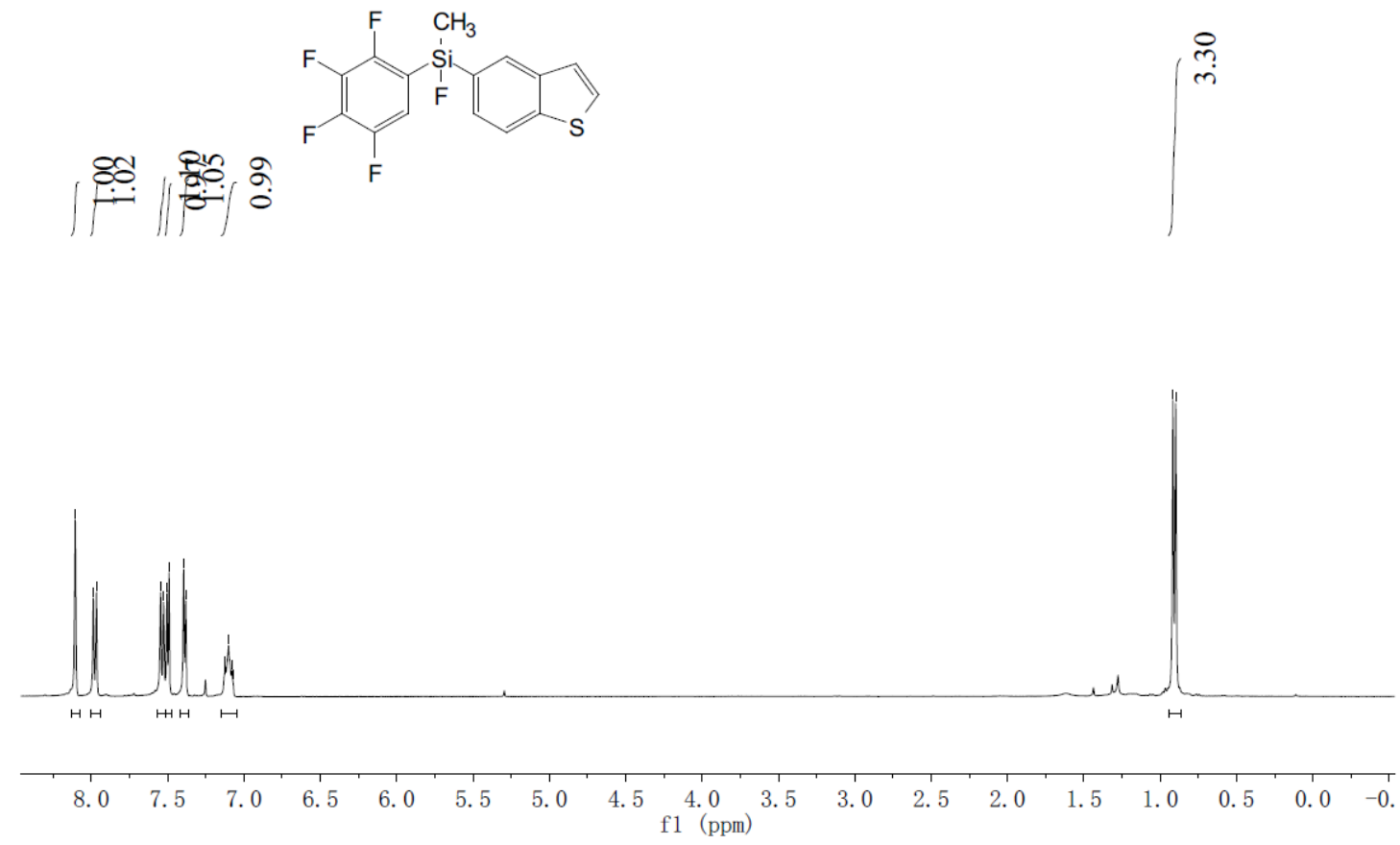

${ }^{1} \mathrm{H}$ NMR $\left(400 \mathrm{MHz}, \mathrm{CDCl}_{3}\right)$ of $\mathbf{6 g}$

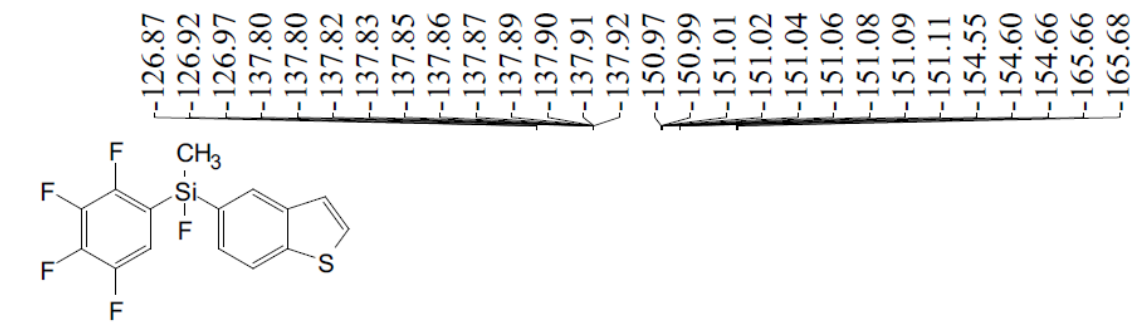

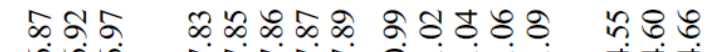

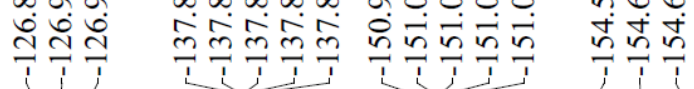
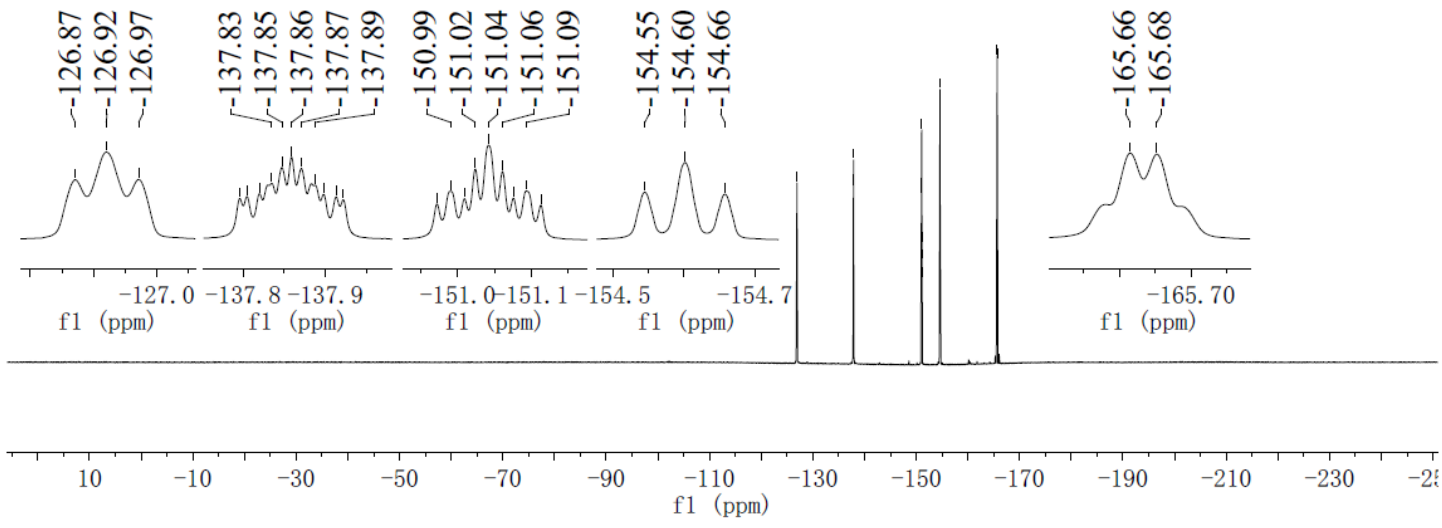

${ }^{19} \mathrm{~F} \mathrm{NMR}\left(376 \mathrm{MHz}, \mathrm{CDCl}_{3}\right)$ of $\mathbf{6 g}$ 


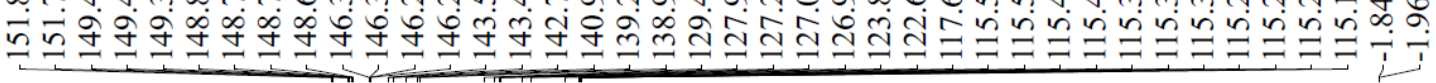

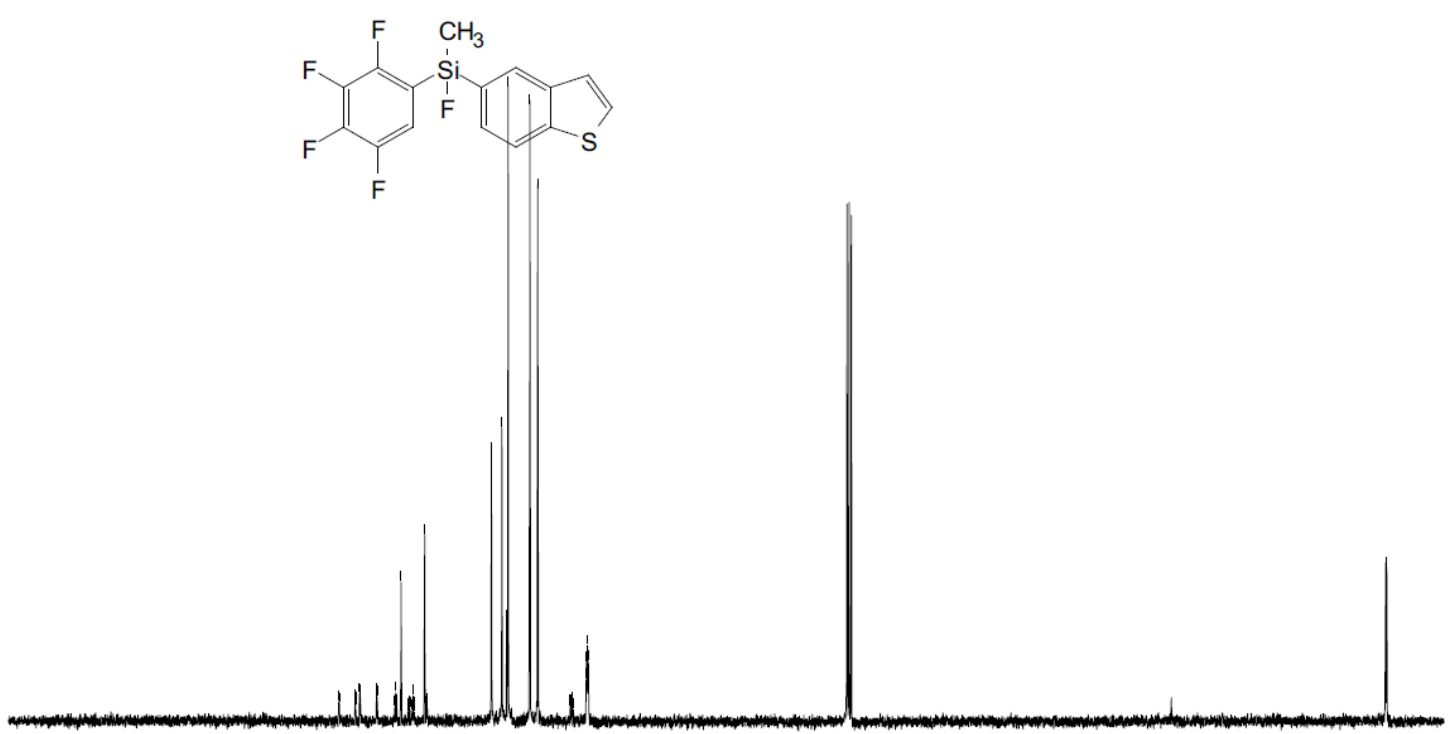

\begin{tabular}{lllllllllllllllllllllll}
\hline 0 & 190 & 180 & 170 & 160 & 150 & 140 & 130 & 120 & 110 & $\begin{array}{l}100 \\
\mathrm{f} 1(\mathrm{ppm})\end{array}$ & 80 & 70 & 60 & 50 & 40 & 30 & 20 & 10 & 0 & -1
\end{tabular}

${ }^{13} \mathrm{C} \mathrm{NMR}\left(101 \mathrm{MHz}, \mathrm{CDCl}_{3}\right)$ of $\mathbf{6 g}$ 


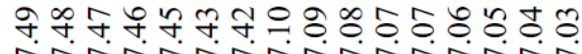

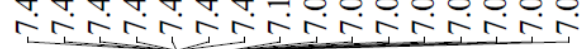

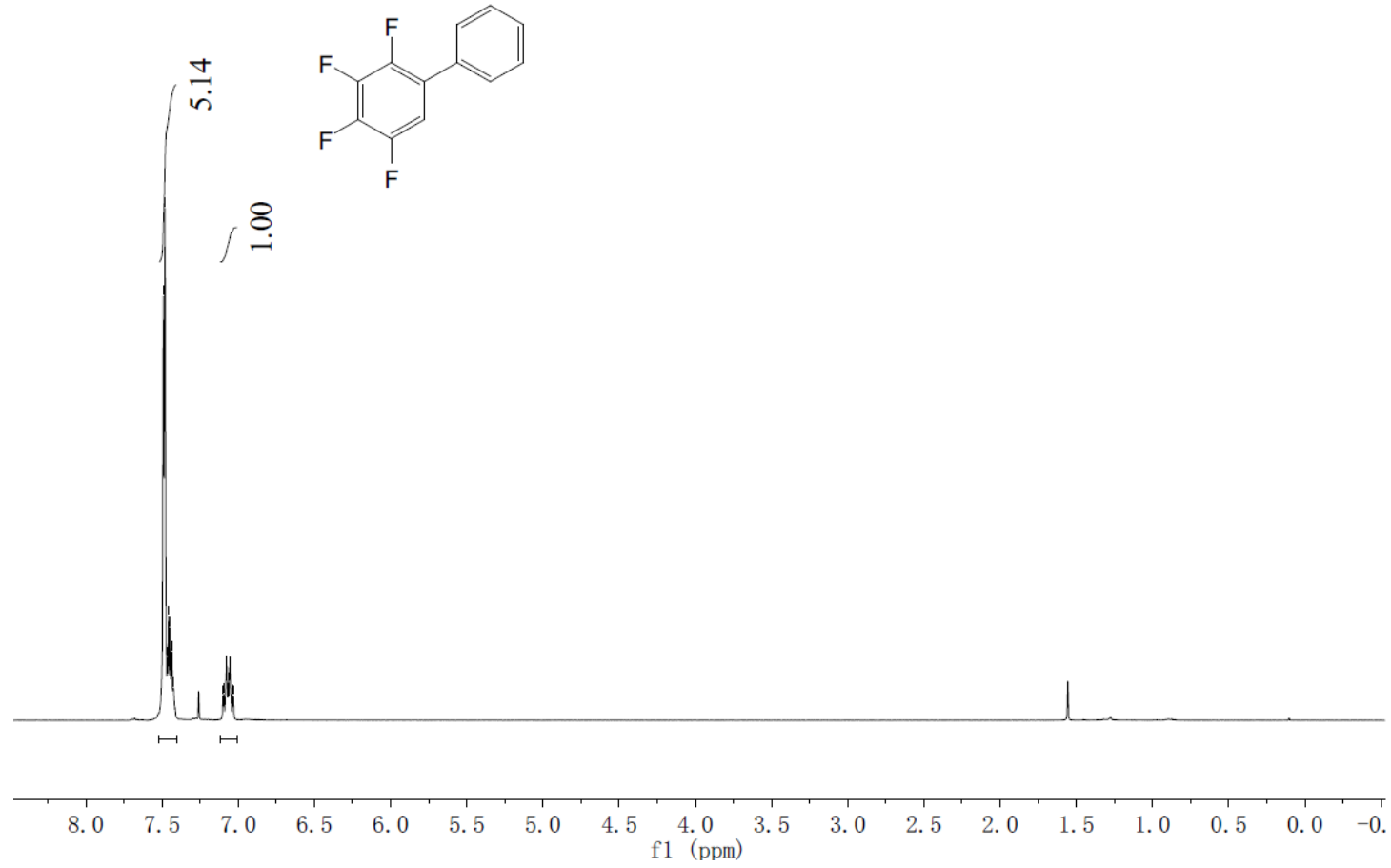

${ }^{1} \mathrm{H} \mathrm{NMR}\left(400 \mathrm{MHz}, \mathrm{CDCl}_{3}\right)$ of $\mathbf{4 a}$

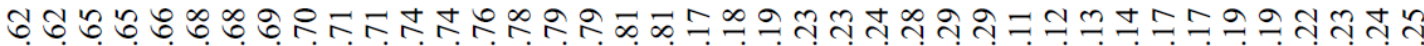

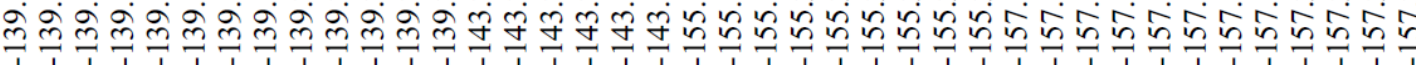<smiles>Fc1cc(-c2ccccc2)c(F)c(F)c1F</smiles>

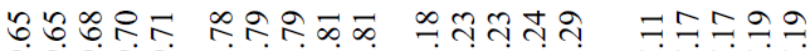

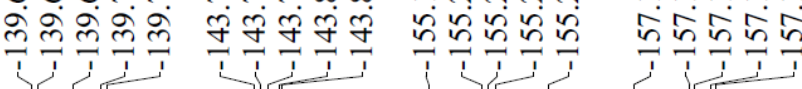

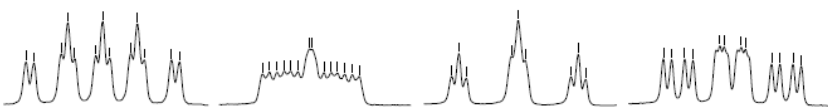

$-139.65-139.75 \quad-143.8-143.9 \quad-155.30-157.15-157.25$
f1 (ppm)
f1 (ppm)
f1 (ppm)
f1 (ppm)

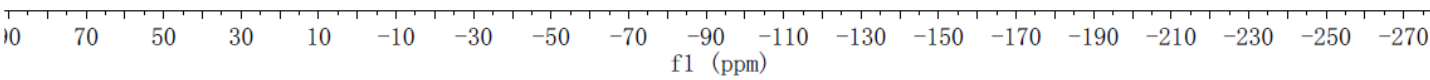

${ }^{19} \mathrm{~F} \mathrm{NMR}\left(376 \mathrm{MHz}, \mathrm{CDCl}_{3}\right)$ of $\mathbf{4 a}$ 

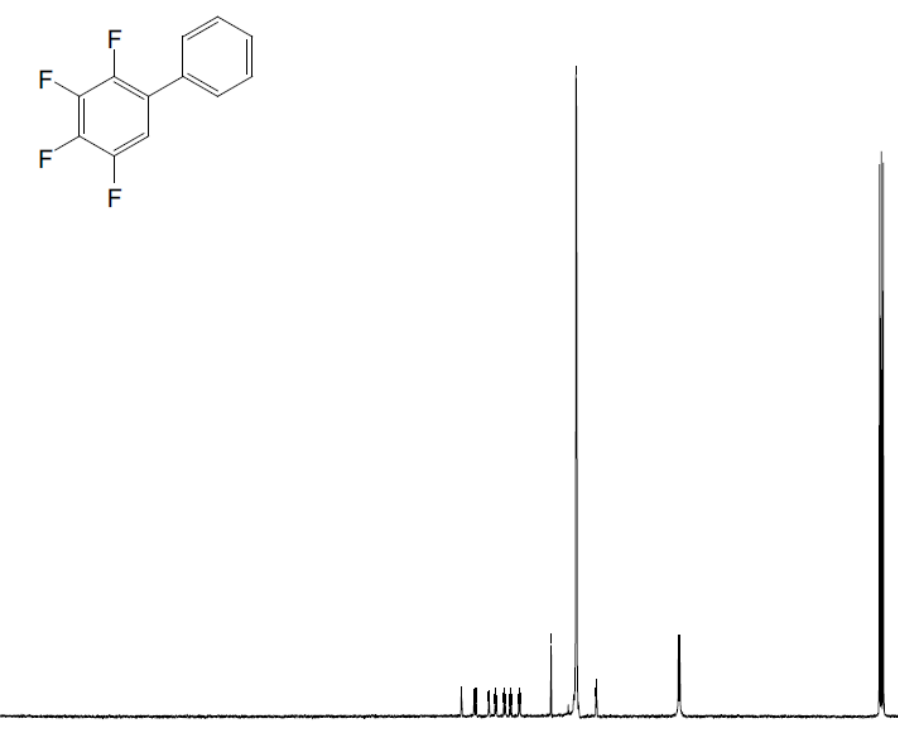

$\begin{array}{lllllllllllllllllllllllll}220 & 210 & 200 & 190 & 180 & 170 & 160 & 150 & 140 & 130 & 120 & \begin{array}{c}110 \\ \mathrm{f} 1\end{array}(\mathrm{ppm}) & & 90 & 80 & 70 & 60 & 50 & 40 & 30 & 20 & 10 & 0 & -10\end{array}$

${ }^{13} \mathrm{C} \mathrm{NMR}\left(101 \mathrm{MHz}, \mathrm{CDCl}_{3}\right)$ of $\mathbf{4 a}$ 


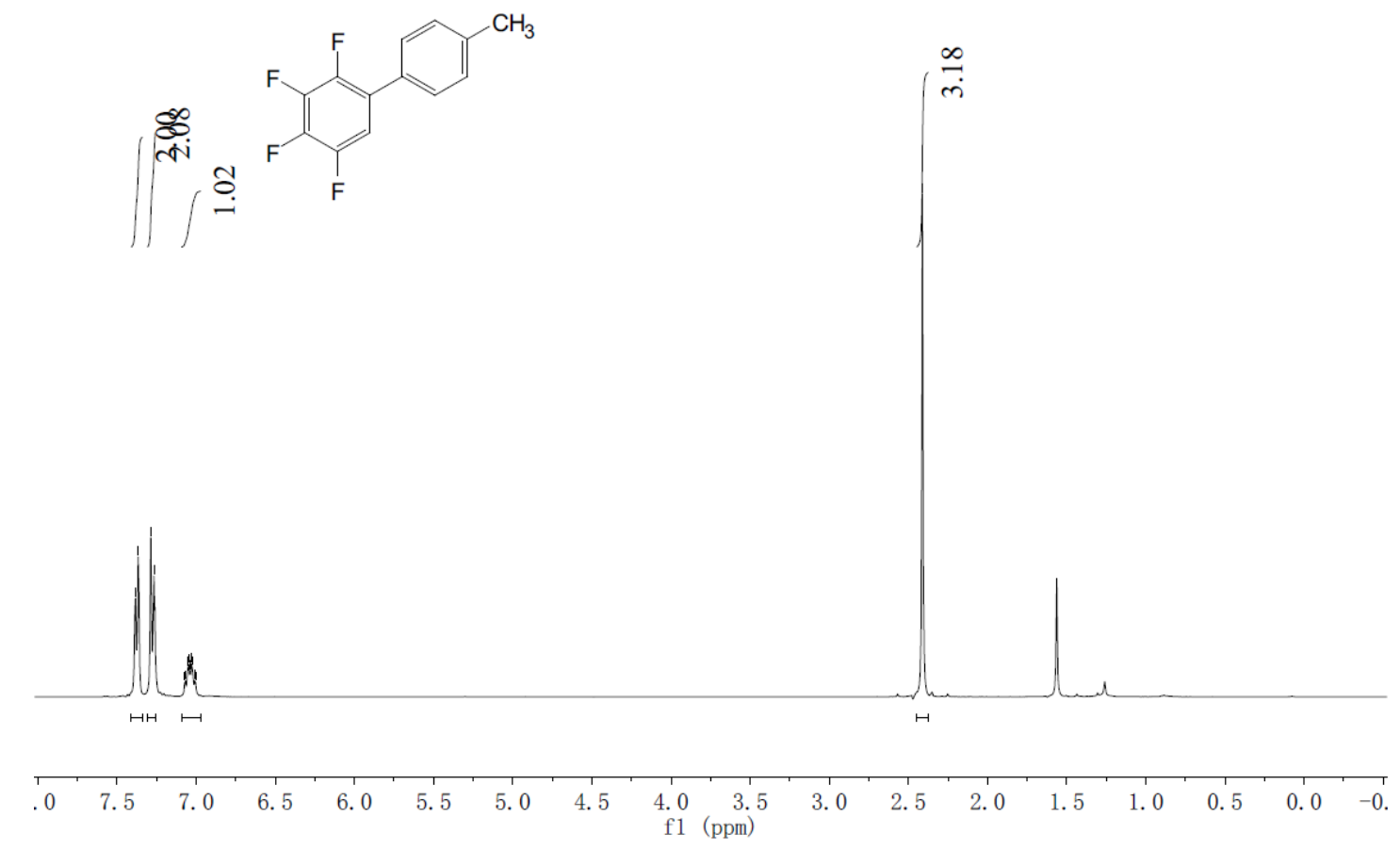

${ }^{1} \mathrm{H}$ NMR $\left(400 \mathrm{MHz}, \mathrm{CDCl}_{3}\right)$ of $\mathbf{4 b}$

б

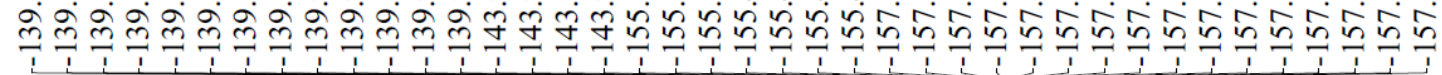<smiles>Cc1ccc(-c2cc(F)c(F)c(F)c2F)cc1</smiles>

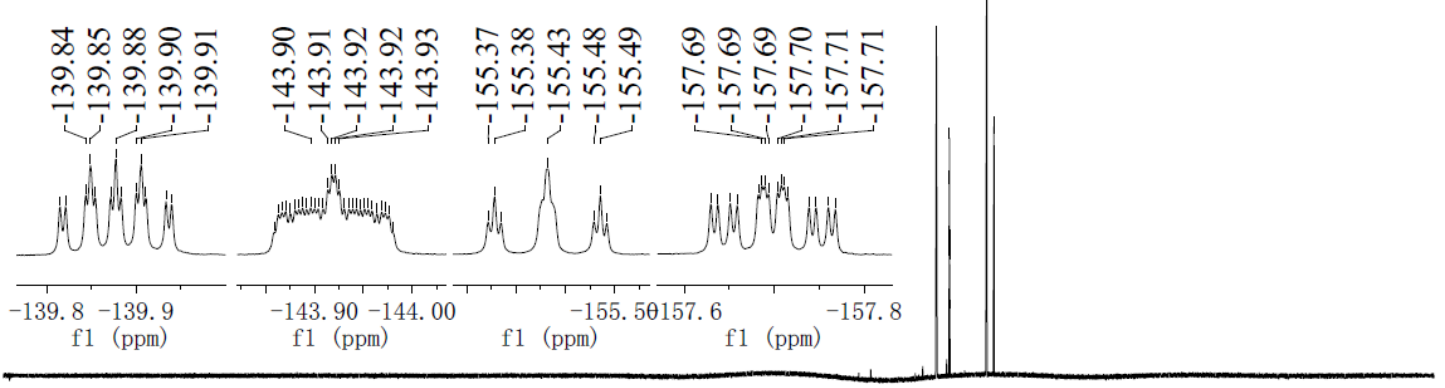

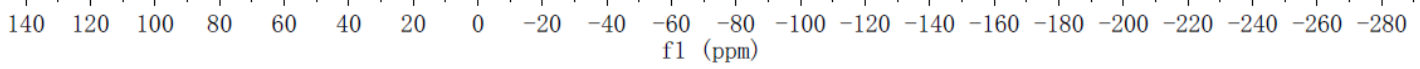
$\left.{ }^{19} \mathrm{~F} \mathrm{NMR} \mathrm{(376} \mathrm{MHz,} \mathrm{CDCl}_{3}\right)$ of $\mathbf{4 b}$ 


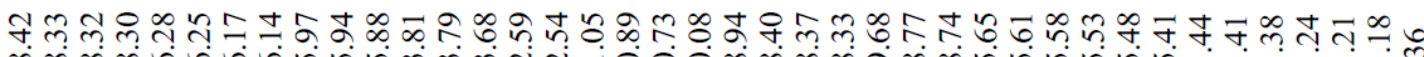

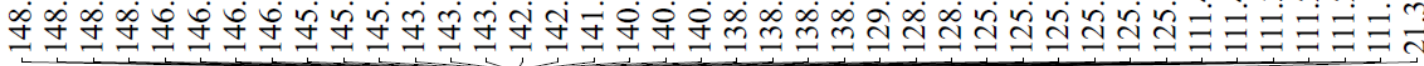
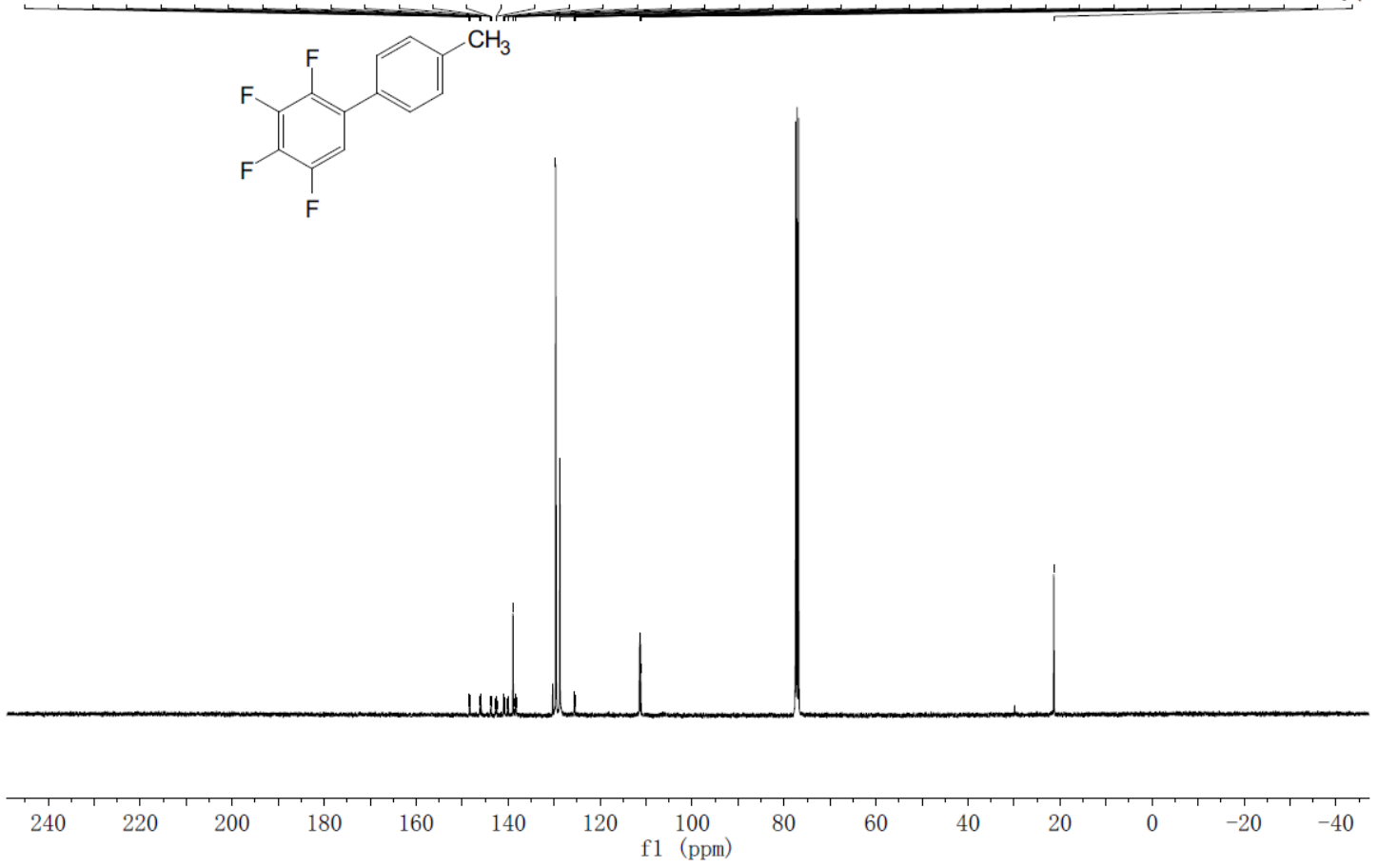

${ }^{13} \mathrm{C} \mathrm{NMR}\left(101 \mathrm{MHz}, \mathrm{CDCl}_{3}\right)$ of $4 \mathrm{~b}$ 


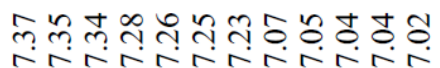

$\stackrel{\text { i }}{\text { ก }}$

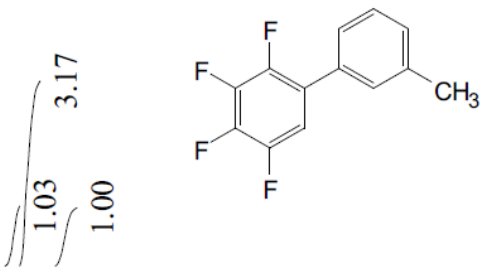

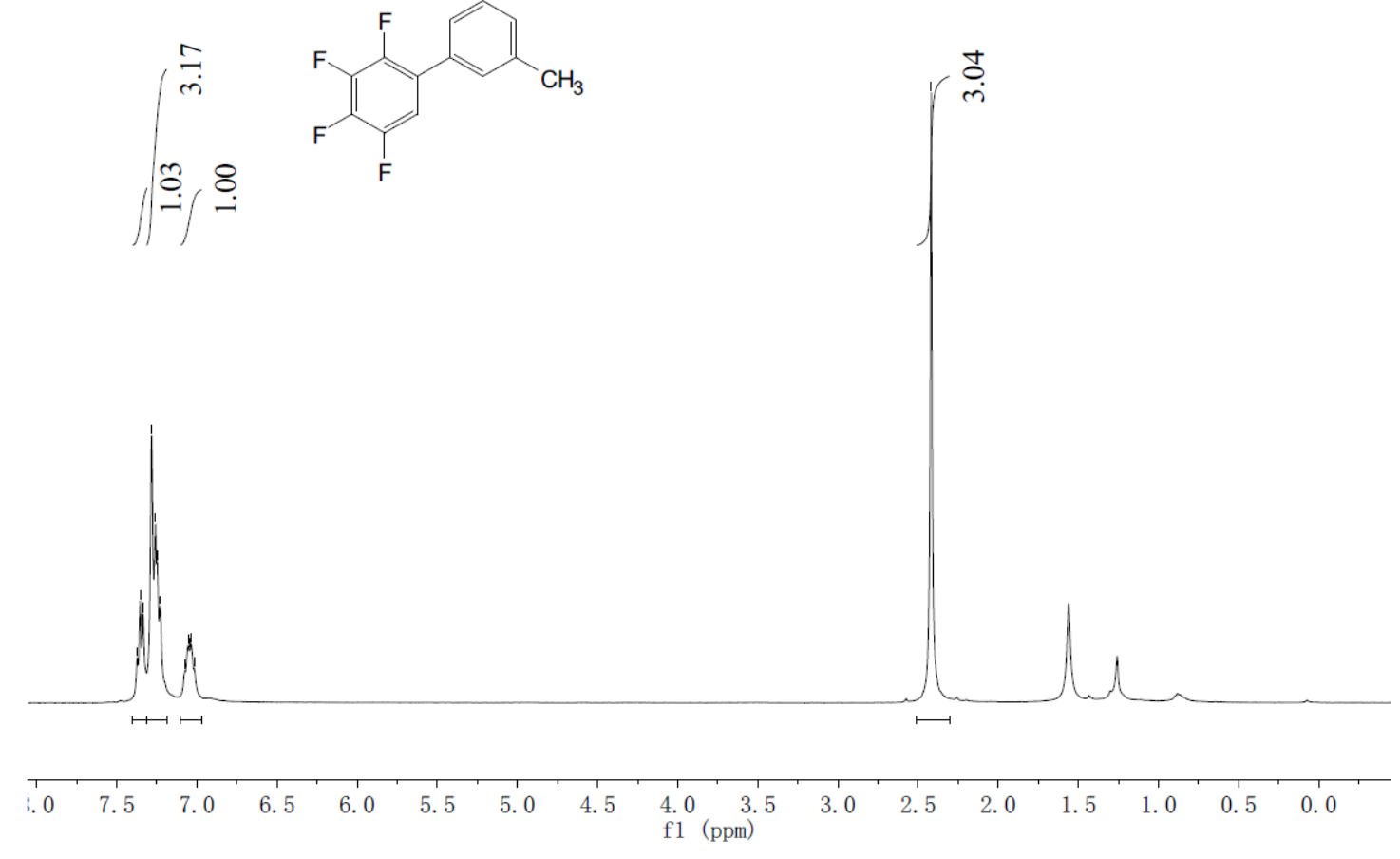

${ }^{1} \mathrm{H} \mathrm{NMR}\left(400 \mathrm{MHz}, \mathrm{CDCl}_{3}\right)$ of $\mathbf{4 c}$

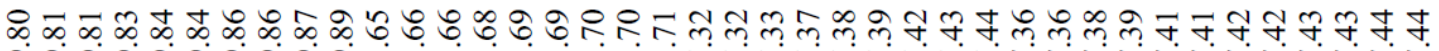
mì<smiles>Cc1cccc(-c2cc(F)c(F)c(F)c2F)c1</smiles>

क

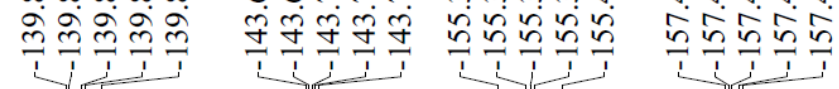

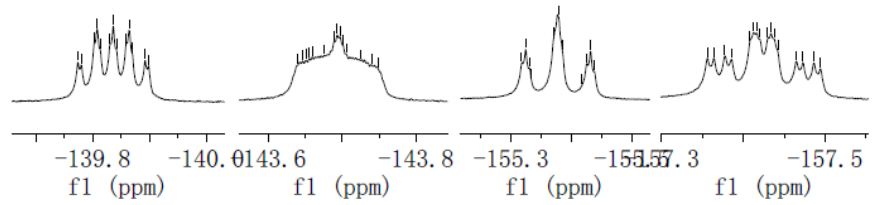

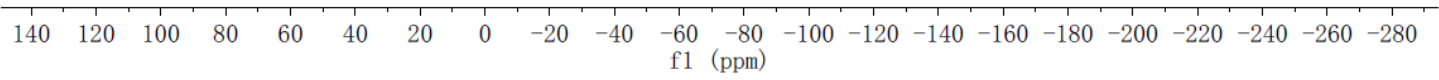

$\left.{ }^{19} \mathrm{~F} \mathrm{NMR} \mathrm{(376} \mathrm{MHz,} \mathrm{CDCl}_{3}\right)$ of $\mathbf{4 c}$ 


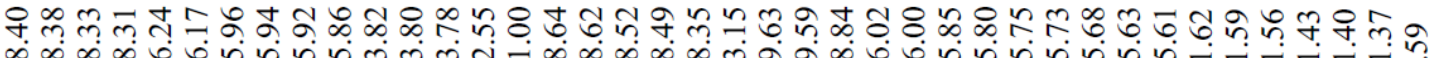

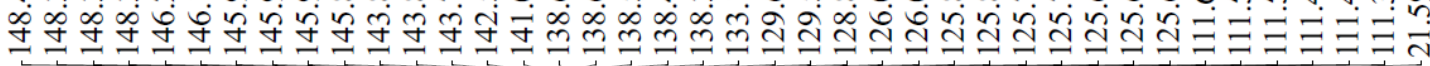<smiles>Cc1cccc(-c2cc(F)c(F)c(F)c2F)c1</smiles>

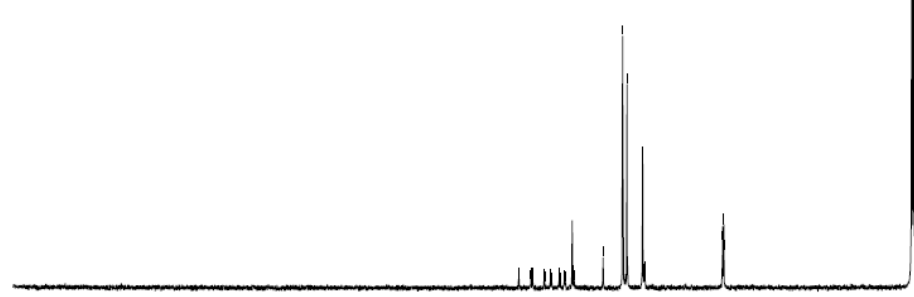

$\begin{array}{llllllllllllllllllllllllll}230 & 220 & 210 & 200 & 190 & 180 & 170 & 160 & 150 & 140 & 130 & 120 & 110 & 100 & 90 & 80 & 70 & 60 & 50 & 40 & 30 & 20 & 10 & 0 & -10\end{array}$ ${ }^{13} \mathrm{C} \mathrm{NMR}\left(101 \mathrm{MHz}, \mathrm{CDCl}_{3}\right)$ of $4 \mathrm{c}$ 

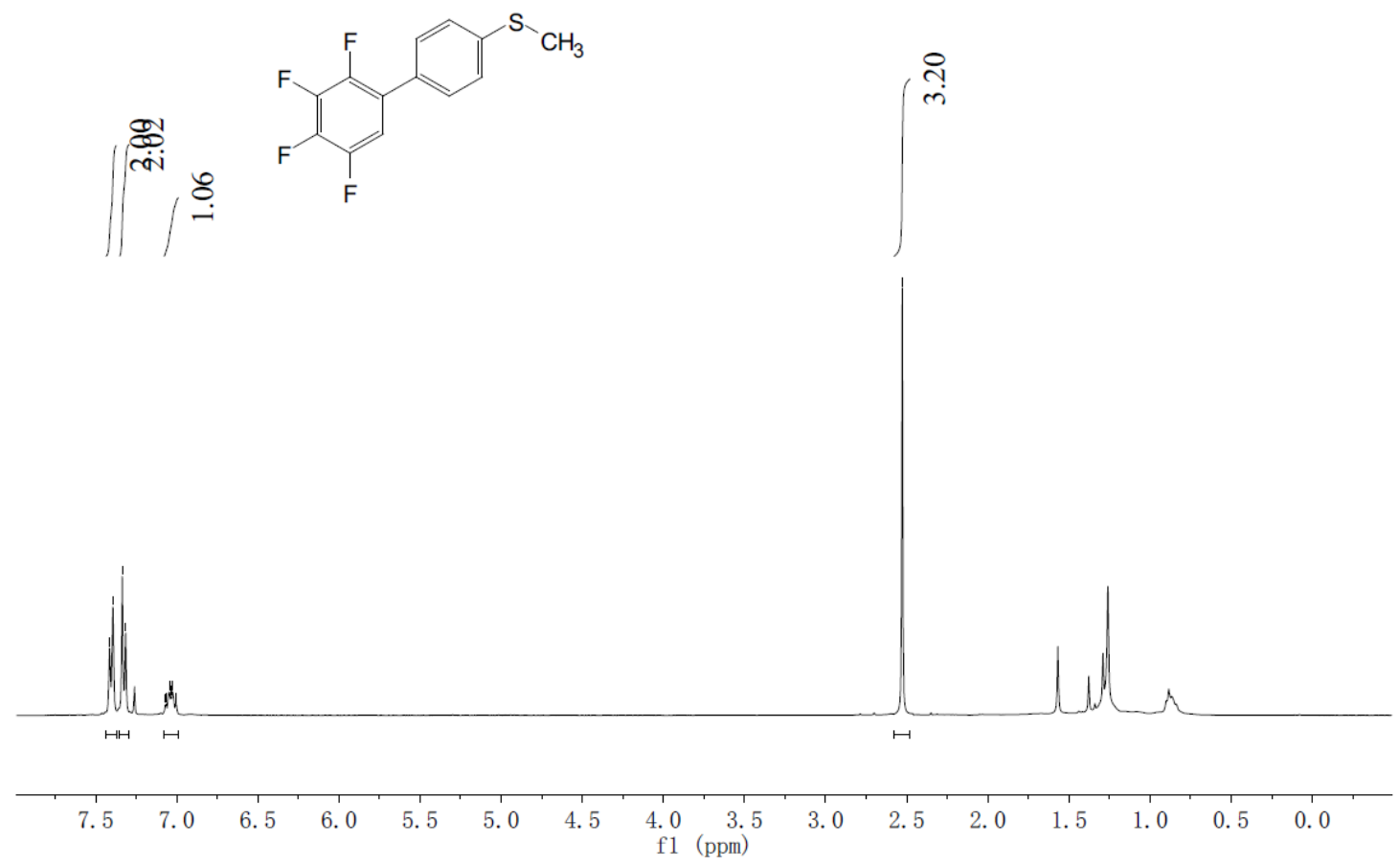

${ }^{1} \mathrm{H}$ NMR $\left(400 \mathrm{MHz}, \mathrm{CDCl}_{3}\right)$ of $\mathbf{4 d}$

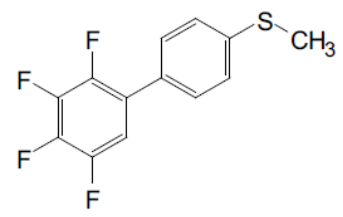

约的员

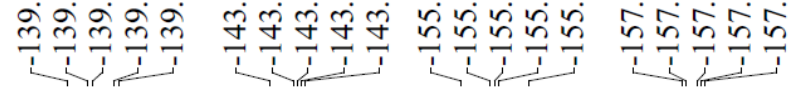

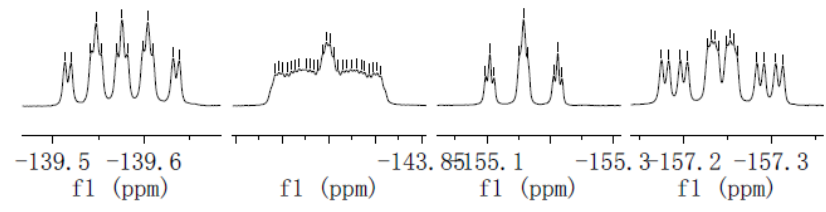

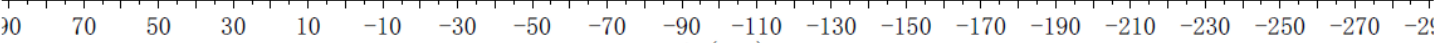
f1 (ppm)

${ }^{19} \mathrm{~F}$ NMR $\left(376 \mathrm{MHz}, \mathrm{CDCl}_{3}\right)$ of $\mathbf{4 d}$ 

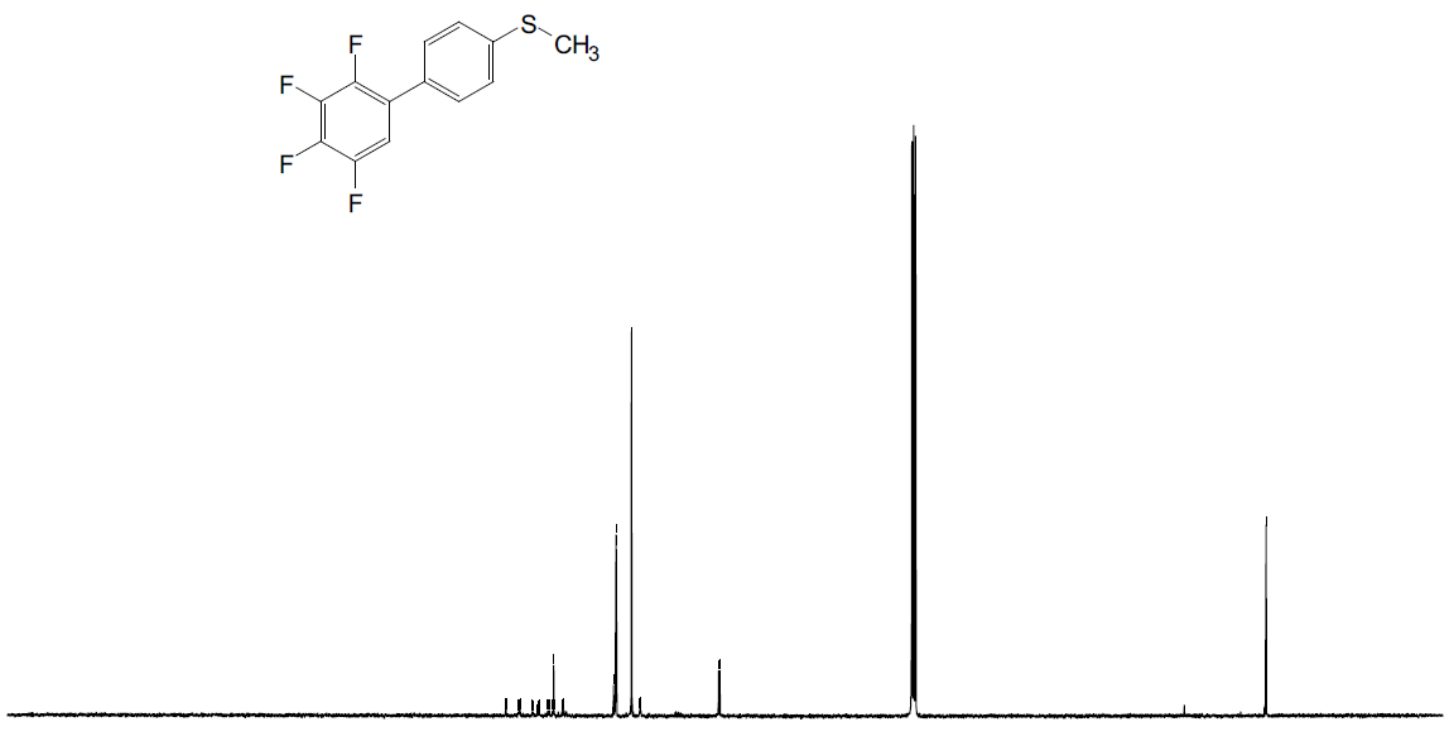

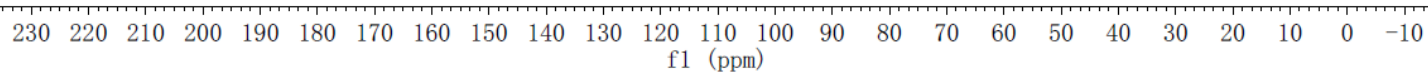
${ }^{13} \mathrm{C} \mathrm{NMR}\left(101 \mathrm{MHz}, \mathrm{CDCl}_{3}\right)$ of $\mathbf{4 d}$ 


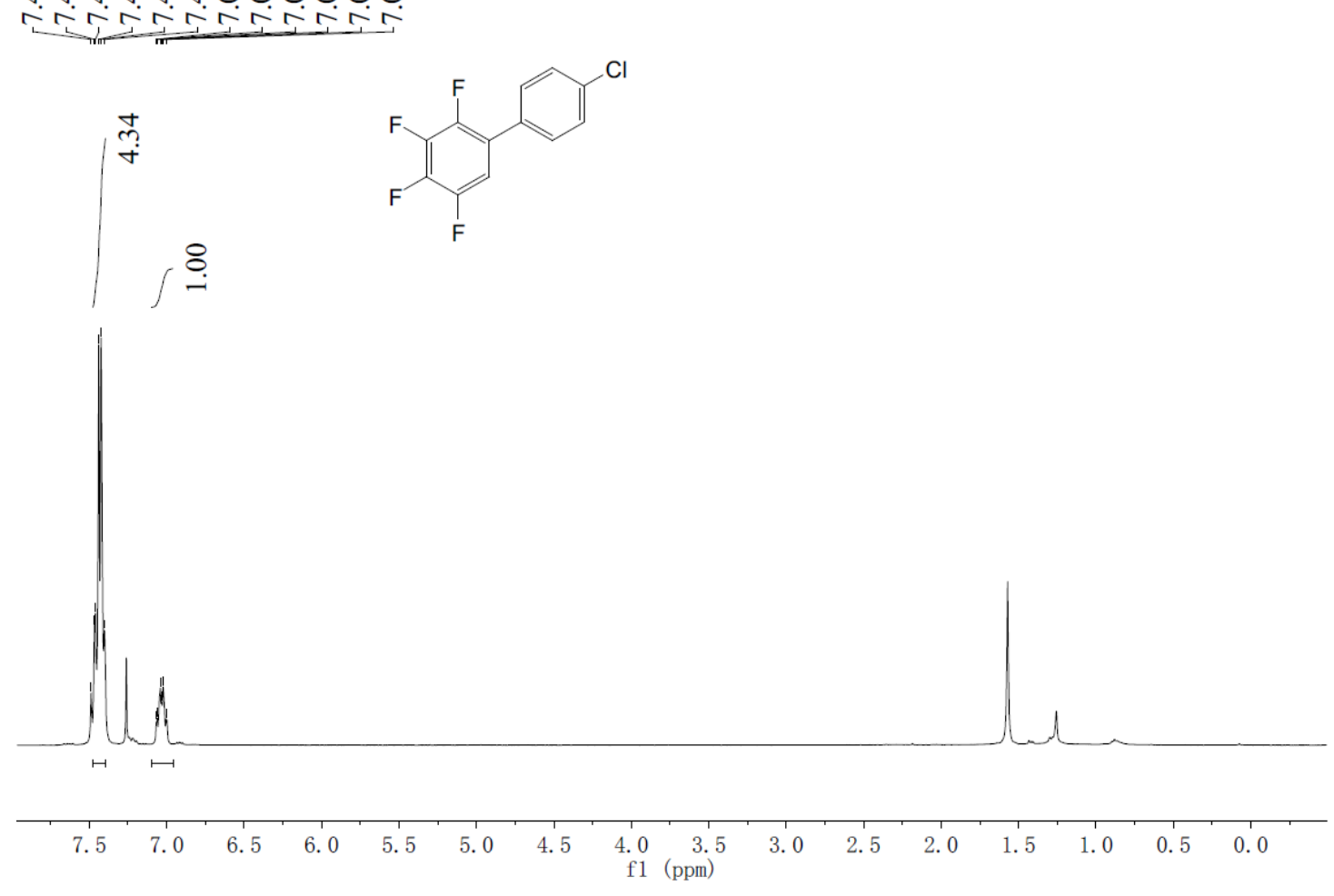

${ }^{1} \mathrm{H} \mathrm{NMR}\left(400 \mathrm{MHz}, \mathrm{CDCl}_{3}\right)$ of $4 \mathrm{e}$

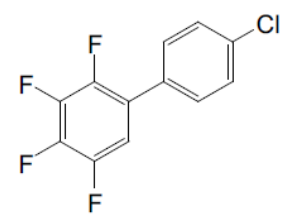

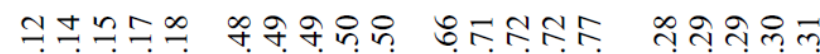

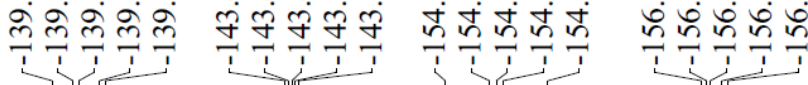

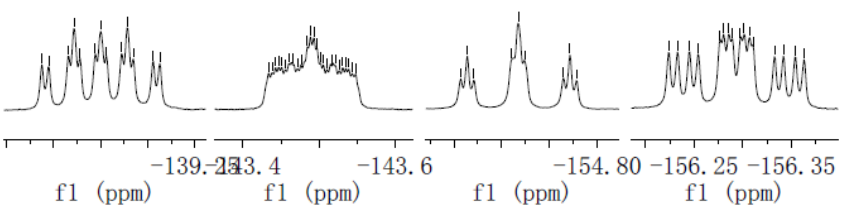

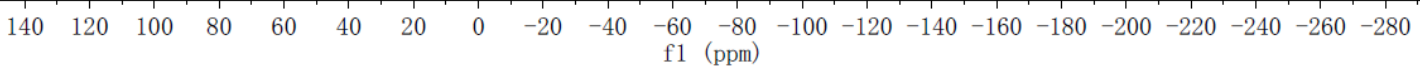

${ }^{19} \mathrm{~F} \mathrm{NMR}\left(376 \mathrm{MHz}, \mathrm{CDCl}_{3}\right)$ of $4 \mathrm{e}$ 


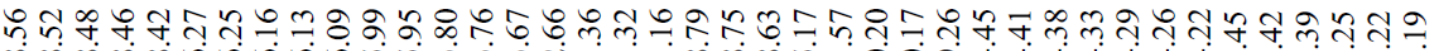

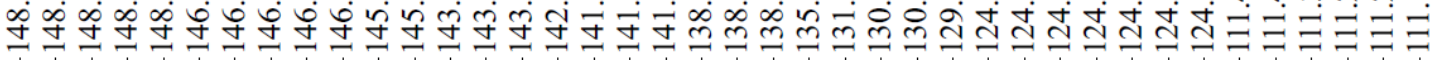<smiles>Fc1cc(-c2ccc(Cl)cc2)c(F)c(F)c1F</smiles>

$\begin{array}{llllllllllllll}250 & 240 & 230 & 220 & 210 & 200 & 190 & 180 & 170 & 160 & 150 & 140 & 130 & 120\end{array}$ f1 (ppm)

${ }^{13} \mathrm{C} \mathrm{NMR}\left(101 \mathrm{MHz}, \mathrm{CDCl}_{3}\right)$ of $4 \mathbf{e}$ 


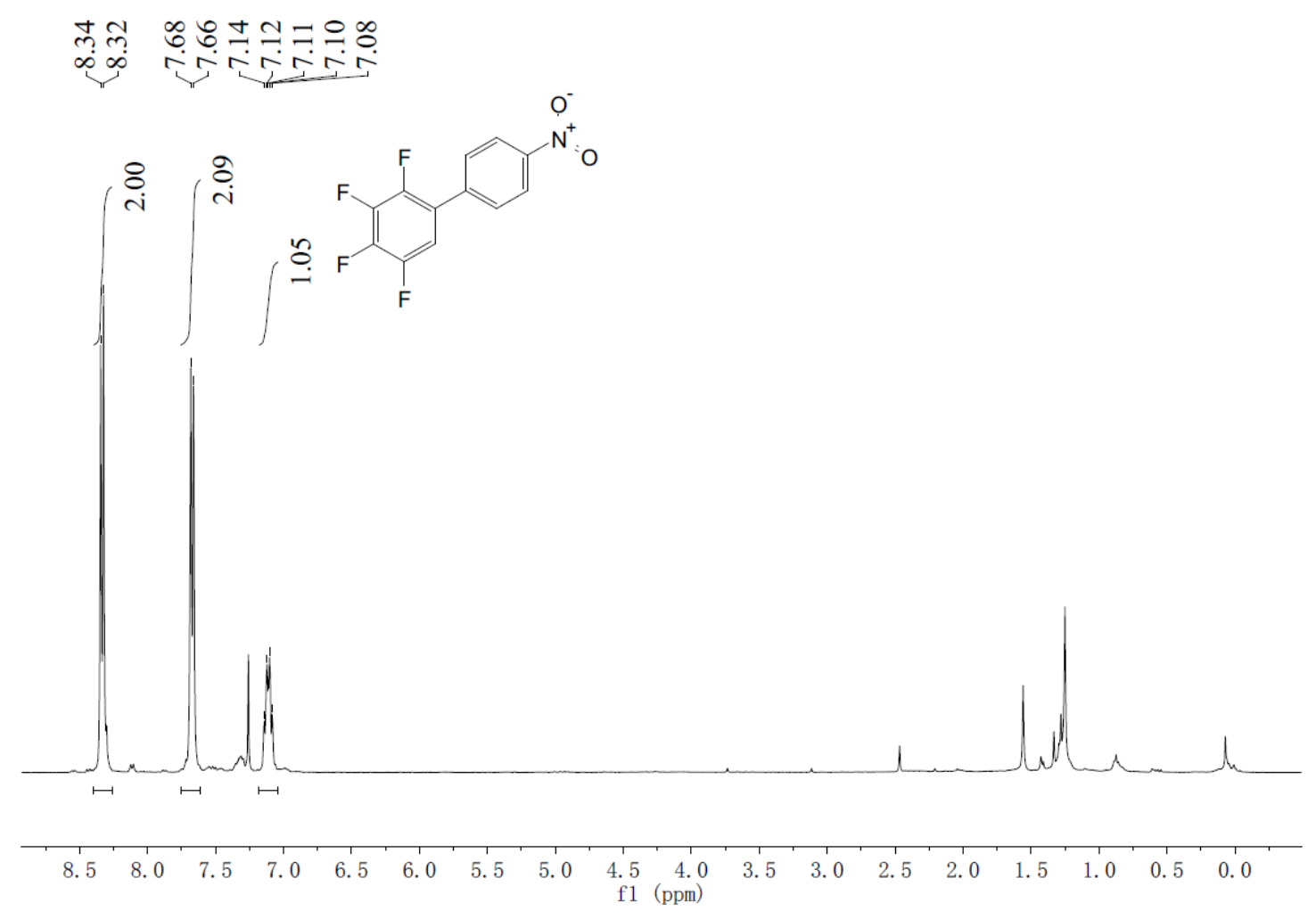

${ }^{1} \mathrm{H} \mathrm{NMR}\left(400 \mathrm{MHz}, \mathrm{CDCl}_{3}\right)$ of $\mathbf{4 f}$

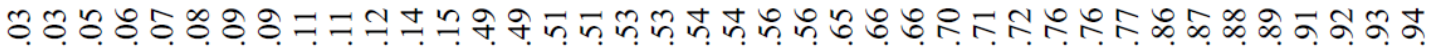

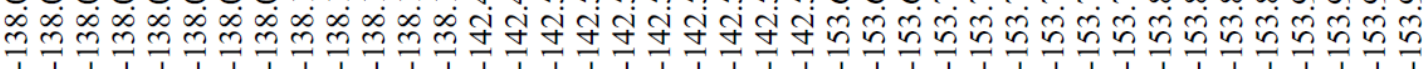

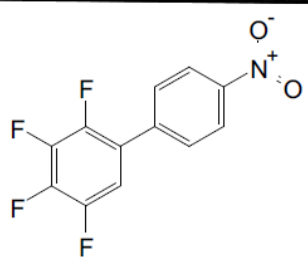

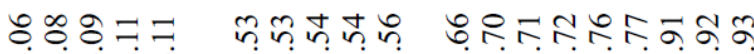

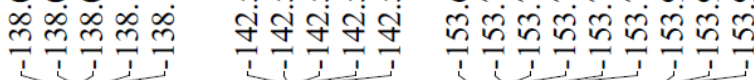

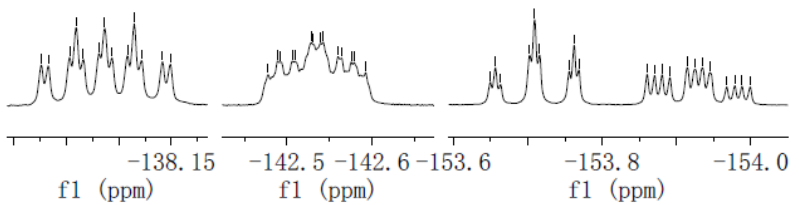

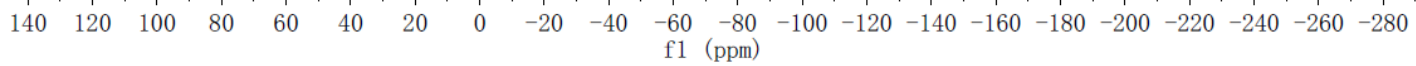

${ }^{19} \mathrm{~F} \mathrm{NMR}\left(376 \mathrm{MHz}, \mathrm{CDCl}_{3}\right)$ of $\mathbf{4 f}$ 
ทิ กำㅇํㅇำ

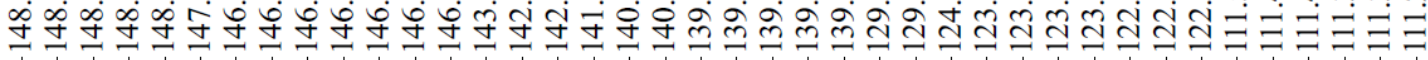

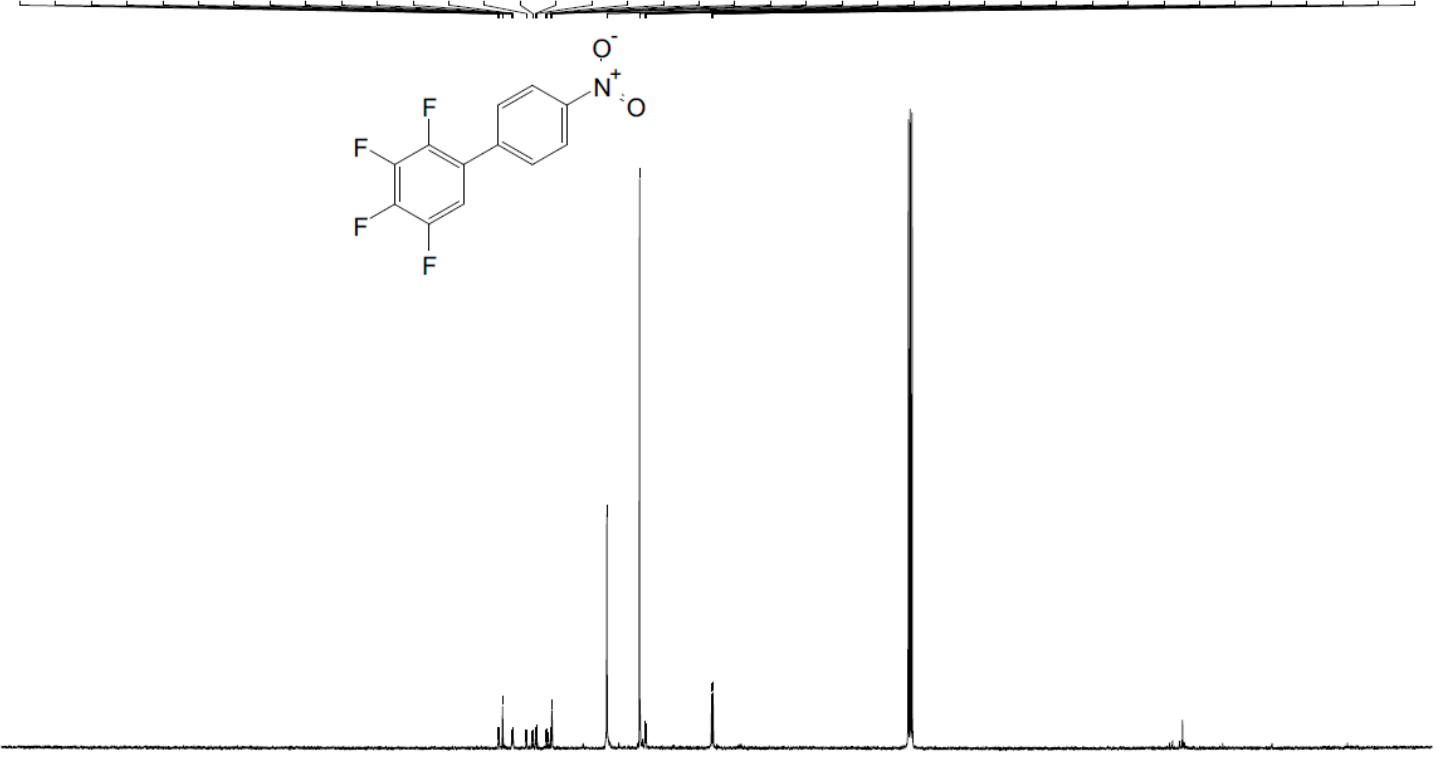

$\begin{array}{lllllllllllllllllllllllllllll}1 & 120 & 220 & 210 & 200 & 190 & 180 & 170 & 160 & 150 & 140 & 130 & 120 & 110 & 100 & 90 & 80 & 70 & 60 & 50 & 40 & 30 & 20 & 10 & 0 & -10\end{array}$

${ }^{13} \mathrm{C}$ NMR $\left(101 \mathrm{MHz}, \mathrm{CDCl}_{3}\right)$ of $\mathbf{4 f}$ 


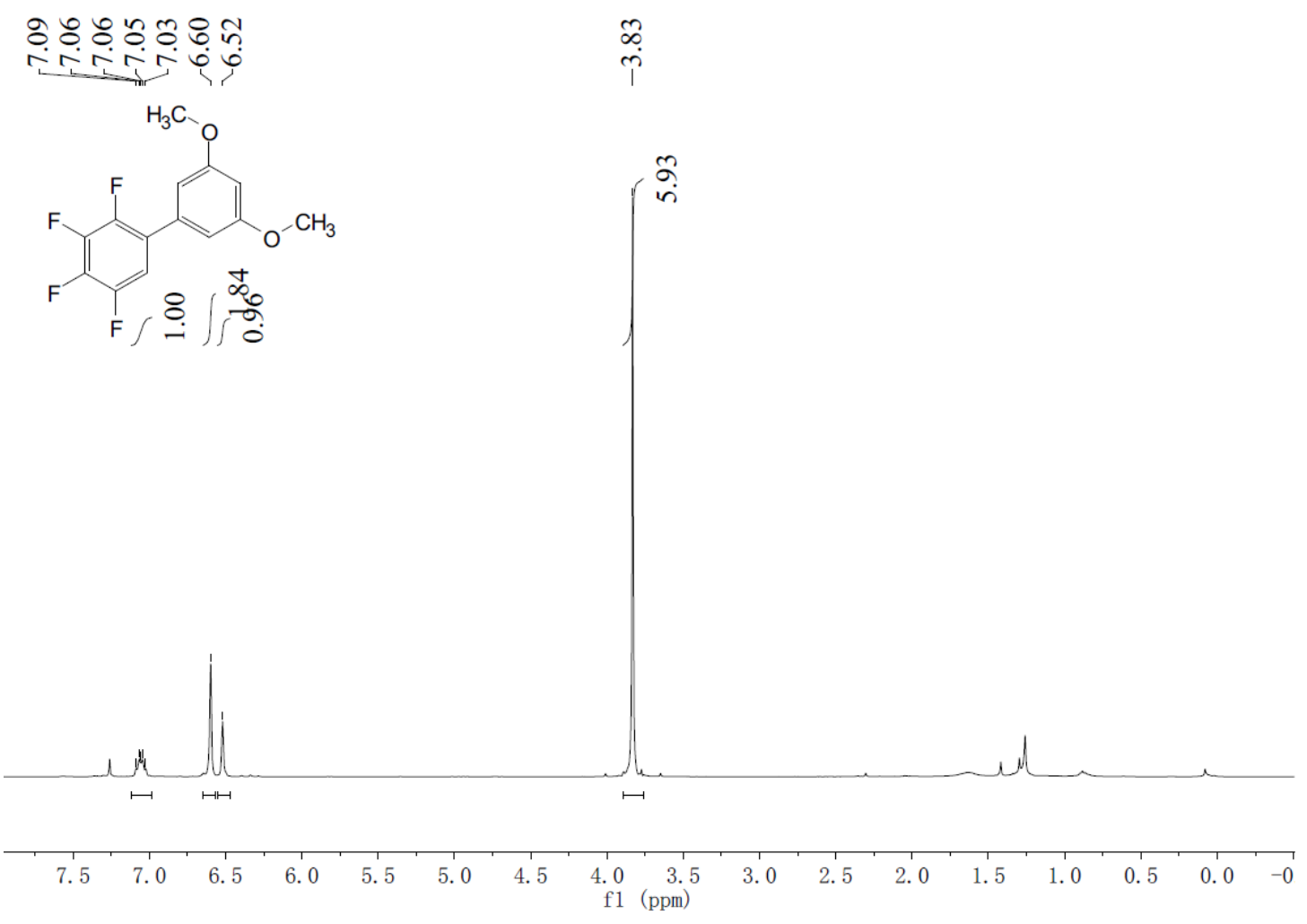

${ }^{1} \mathrm{H} \mathrm{NMR}\left(400 \mathrm{MHz}, \mathrm{CDCl}_{3}\right)$ of $\mathbf{4 g}$

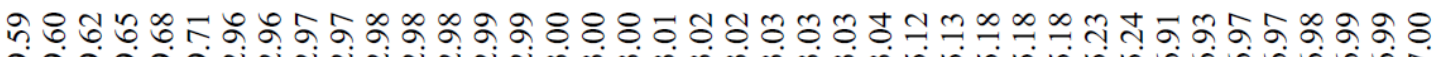

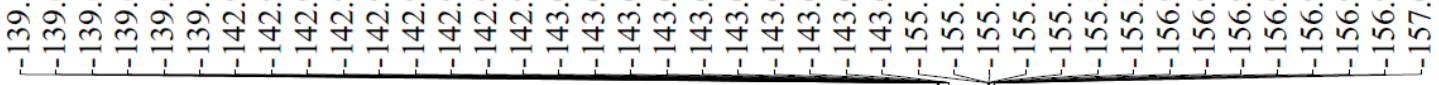

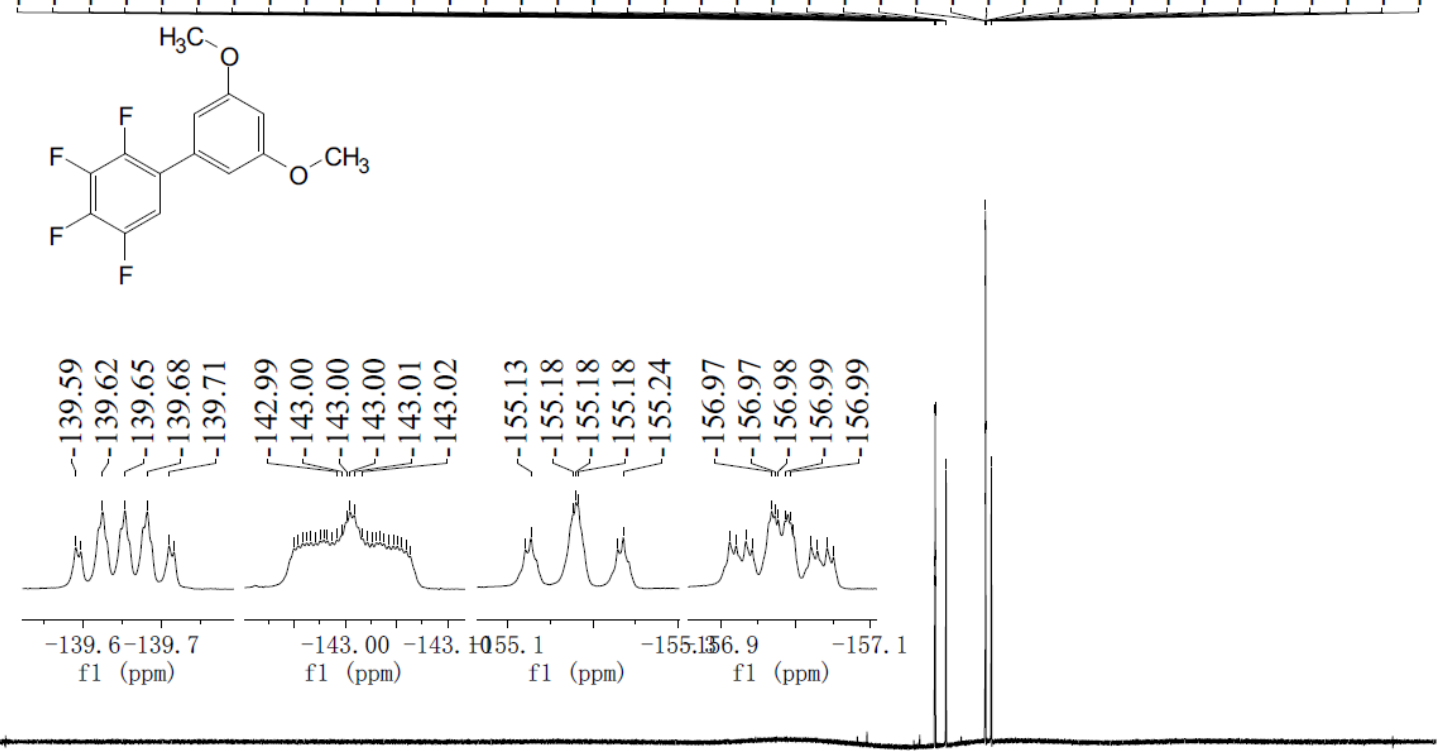

$\begin{array}{lllllllllllllllllllllll}140 & 120 & 100 & 80 & 60 & 40 & 20 & 0 & -20 & -40 & -60 & -80 & -100 & -120 & -140 & -160 & -180 & -200 & -220 & -240 & -260 & -280 \\ \text { f1 } & (\mathrm{ppm})\end{array}$

${ }^{19} \mathrm{~F} \mathrm{NMR}\left(376 \mathrm{MHz}, \mathrm{CDCl}_{3}\right)$ of $\mathbf{4 g}$ 
\&

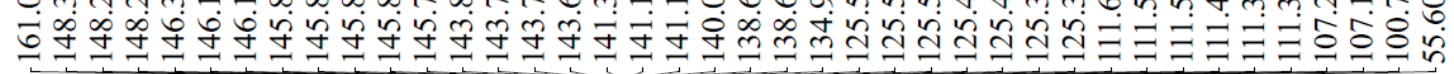

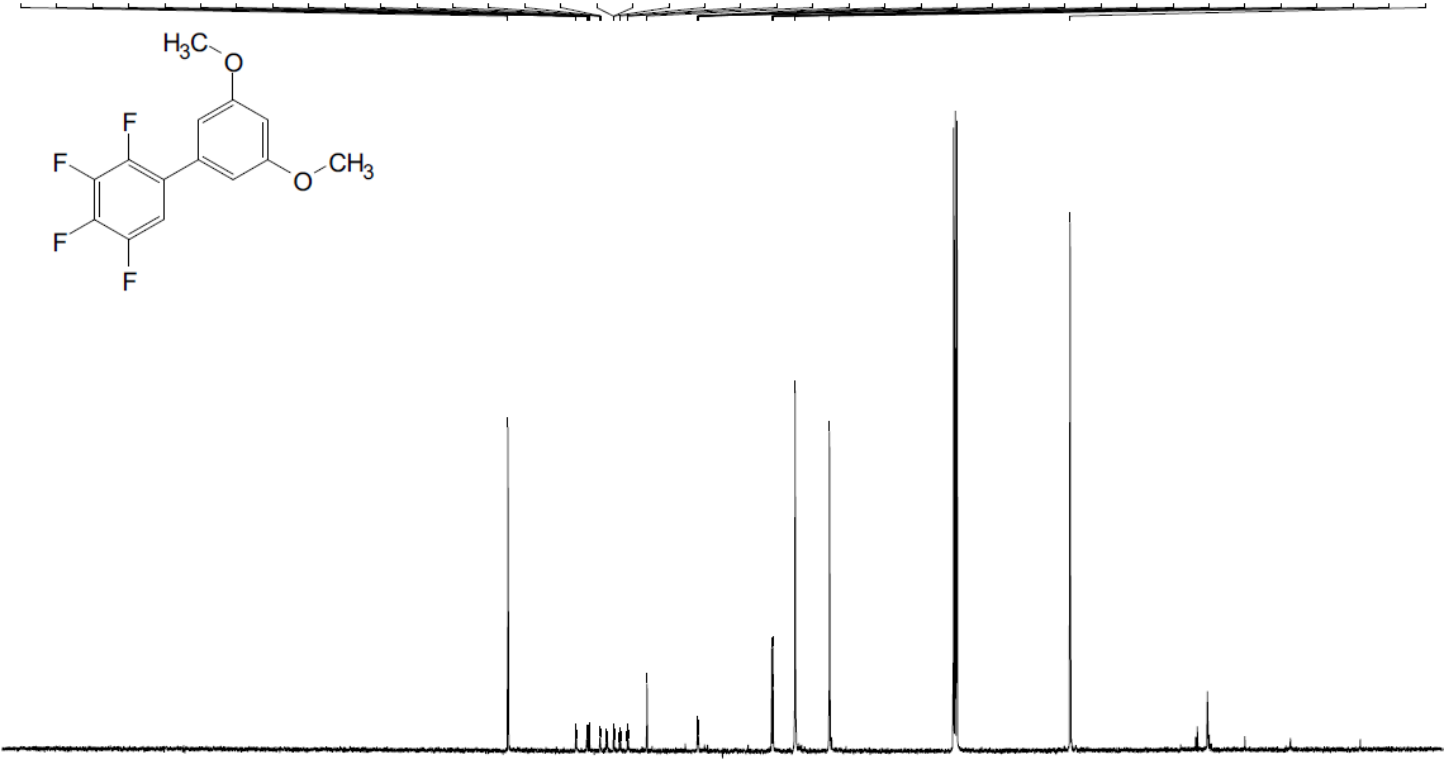

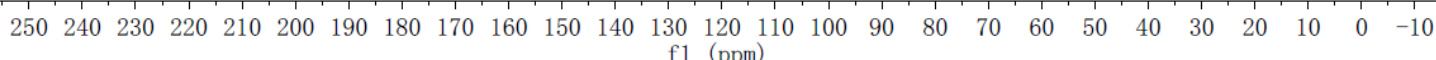
${ }^{13} \mathrm{C}$ NMR $\left(101 \mathrm{MHz}, \mathrm{CDCl}_{3}\right)$ of $\mathbf{4 g}$ 


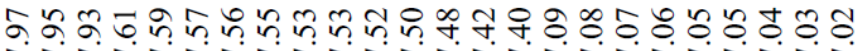

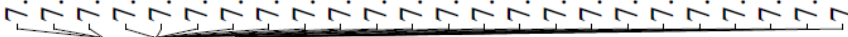

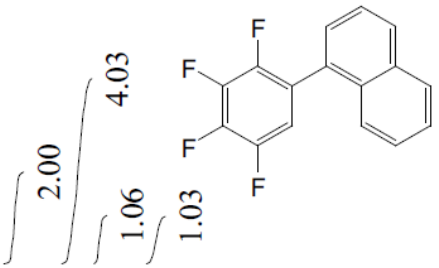

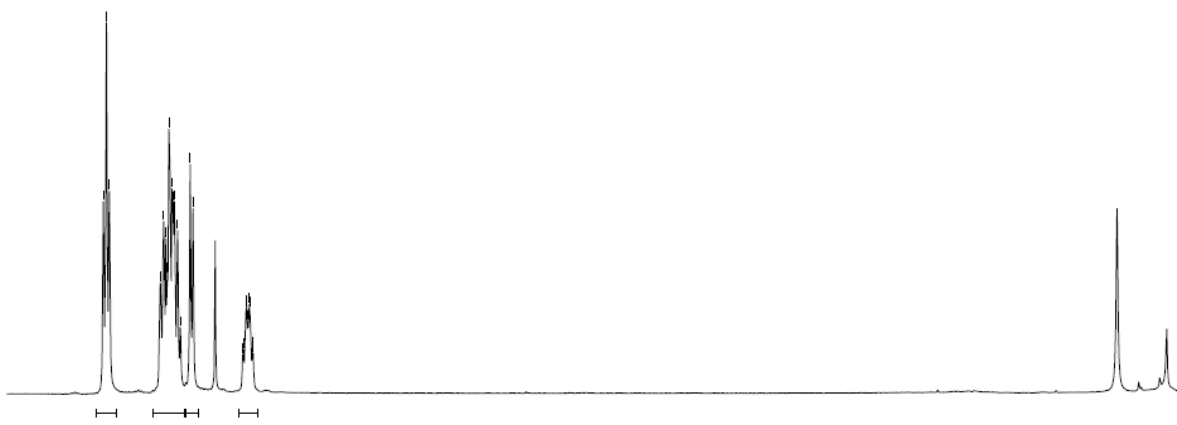

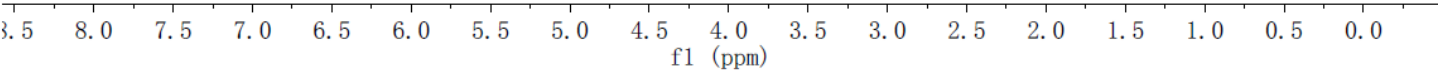

${ }^{1} \mathrm{H} \mathrm{NMR}\left(400 \mathrm{MHz}, \mathrm{CDCl}_{3}\right)$ of $\mathbf{4 h}$

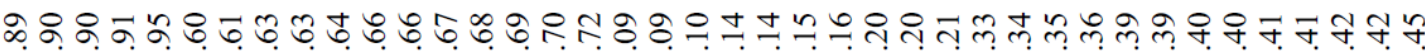

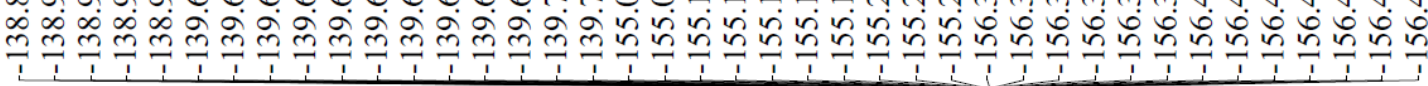<smiles>Fc1cc(-c2cccc3ccccc23)c(F)c(F)c1F</smiles>

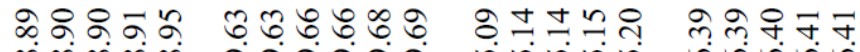

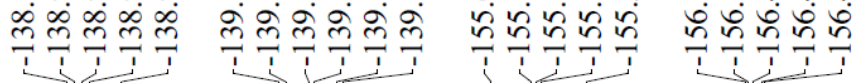

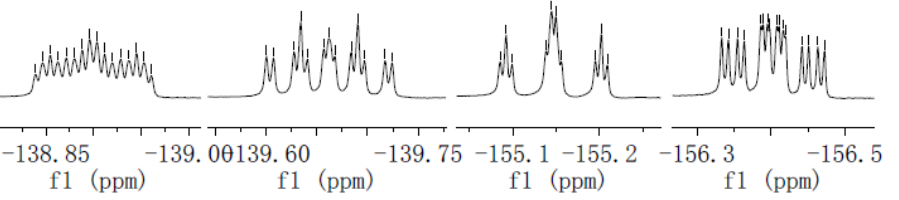

$\begin{array}{llllllllllllllllllllll}140 & 120 & 100 & 80 & 60 & 40 & 20 & 0 & -20 & -40 & -60 & -80 & -100 & -120 & -140 & -160 & -180 & -200 & -220 & -240 & -260 & -280\end{array}$ f1 (ppm)

${ }^{19} \mathrm{~F} \mathrm{NMR}\left(376 \mathrm{MHz}, \mathrm{CDCl}_{3}\right)$ of $\mathbf{4 h}$ 


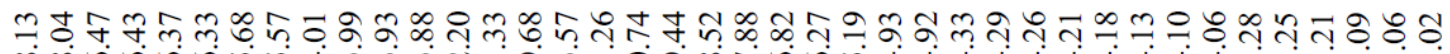

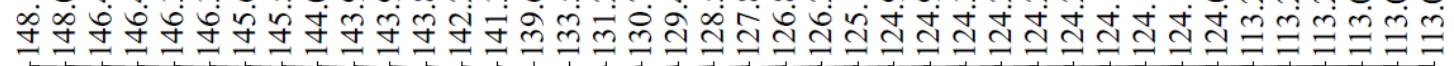<smiles>Fc1ccc(-c2cccc3ccccc23)c(F)c1F</smiles>
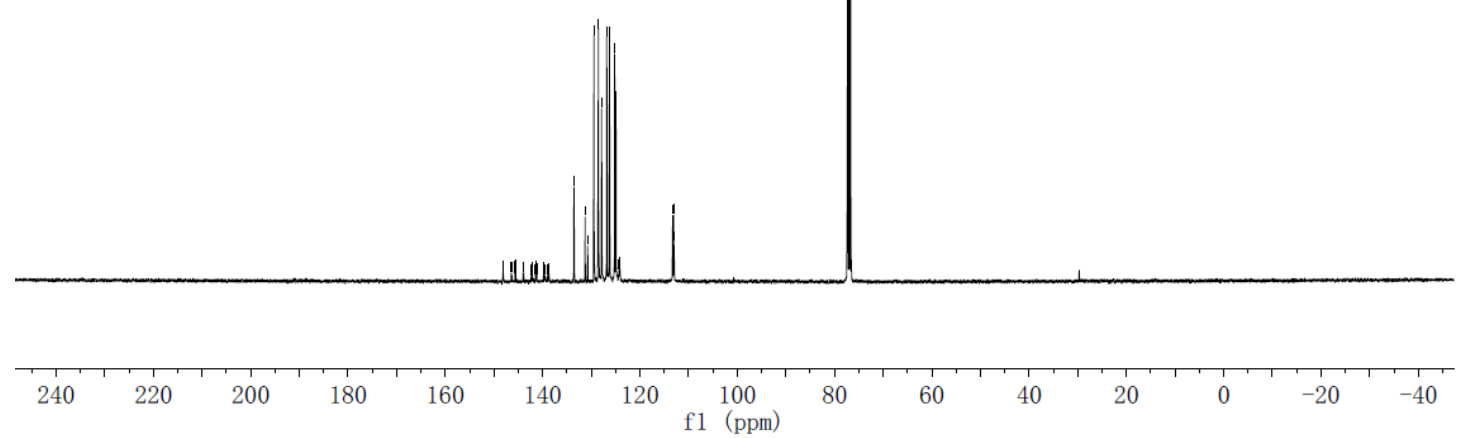

${ }^{13} \mathrm{C} \mathrm{NMR}\left(101 \mathrm{MHz}, \mathrm{CDCl}_{3}\right)$ of $\mathbf{4 h}$ 


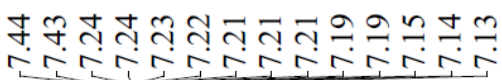

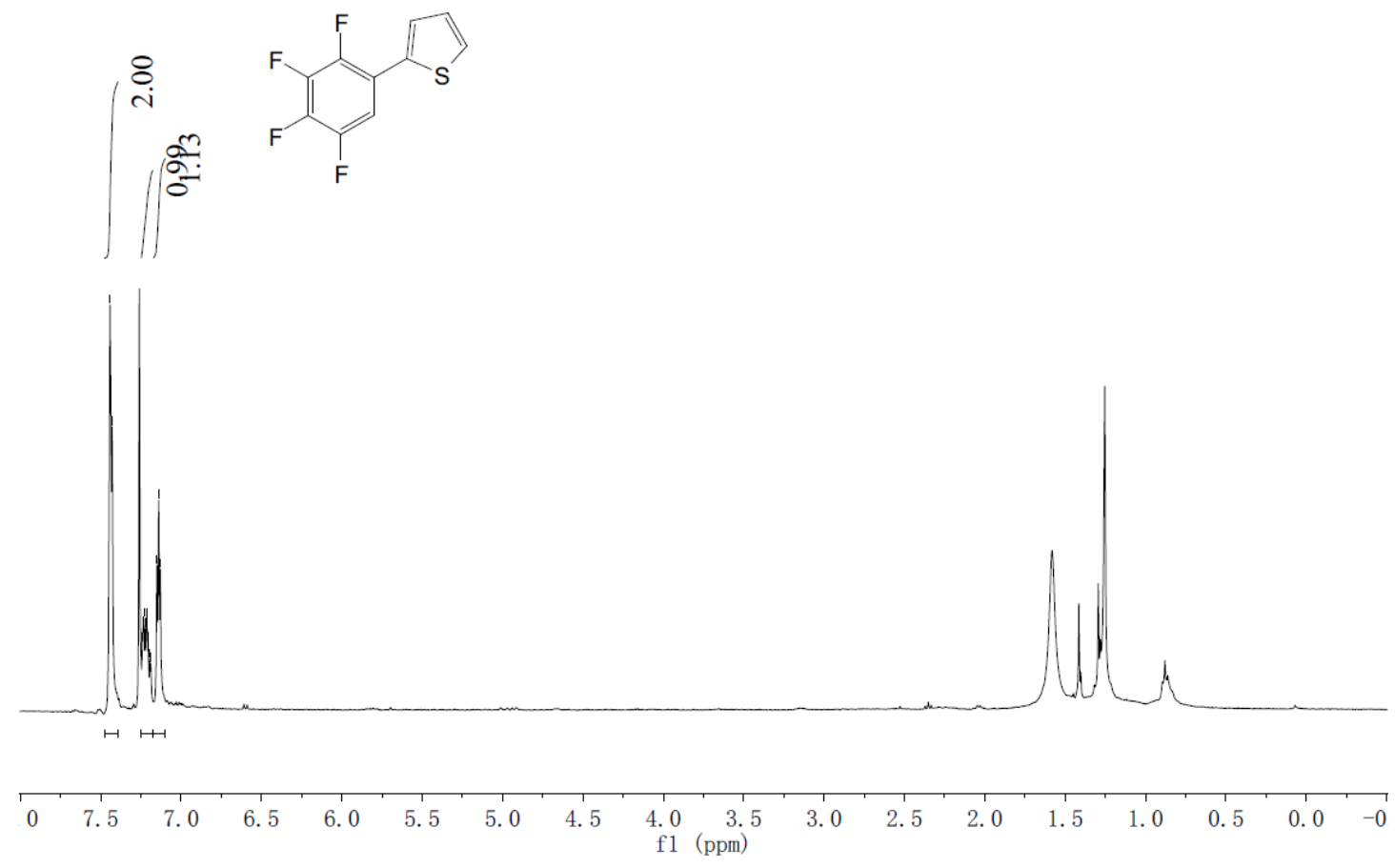

${ }^{1} \mathrm{H} \mathrm{NMR}\left(400 \mathrm{MHz}, \mathrm{CDCl}_{3}\right)$ of $\mathbf{4 i}$

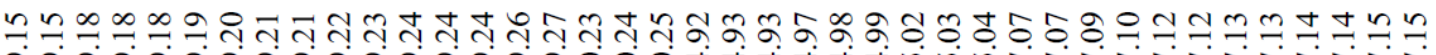

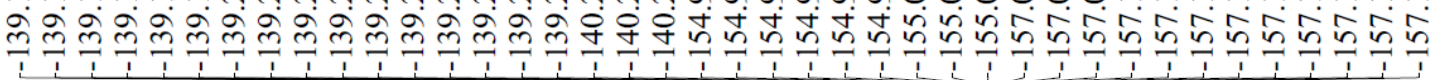<smiles>Fc1cc(-c2cccs2)c(F)c(F)c1F</smiles>

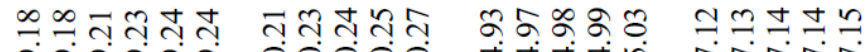

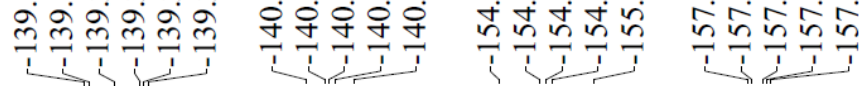

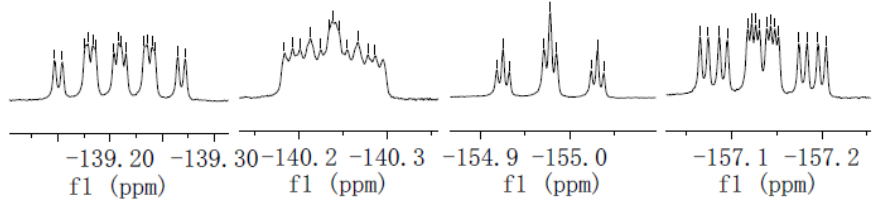

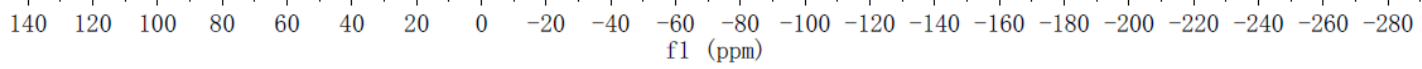

${ }^{19} \mathrm{~F} \mathrm{NMR}\left(376 \mathrm{MHz}, \mathrm{CDCl}_{3}\right)$ of $\mathbf{4 i}$ 


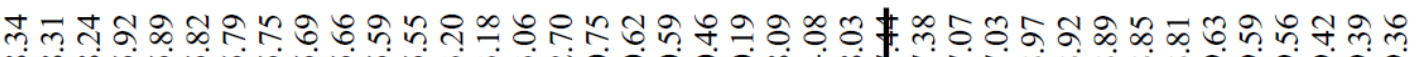

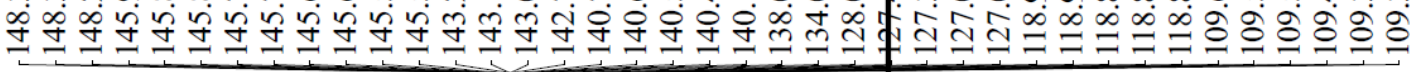<smiles>Fc1cc(-c2cccs2)c(F)c(F)c1F</smiles>

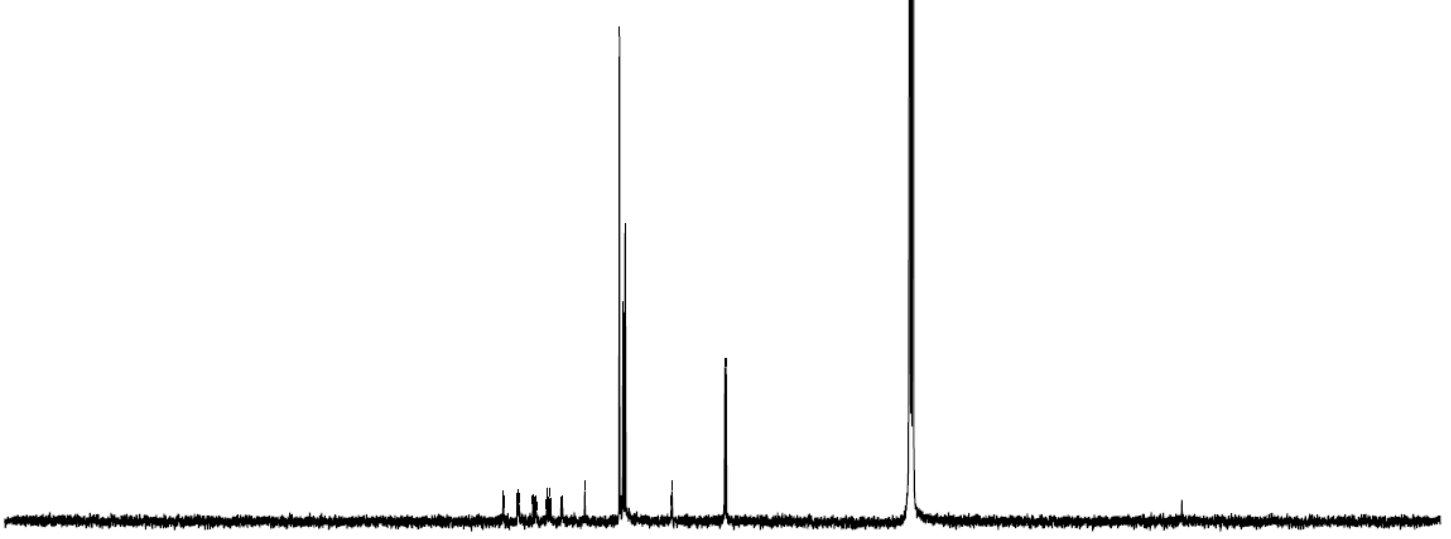

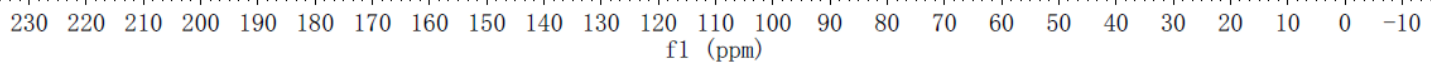

${ }^{13} \mathrm{C}$ NMR $\left(101 \mathrm{MHz}, \mathrm{CDCl}_{3}\right)$ of $\mathbf{4} \mathbf{i}$ 